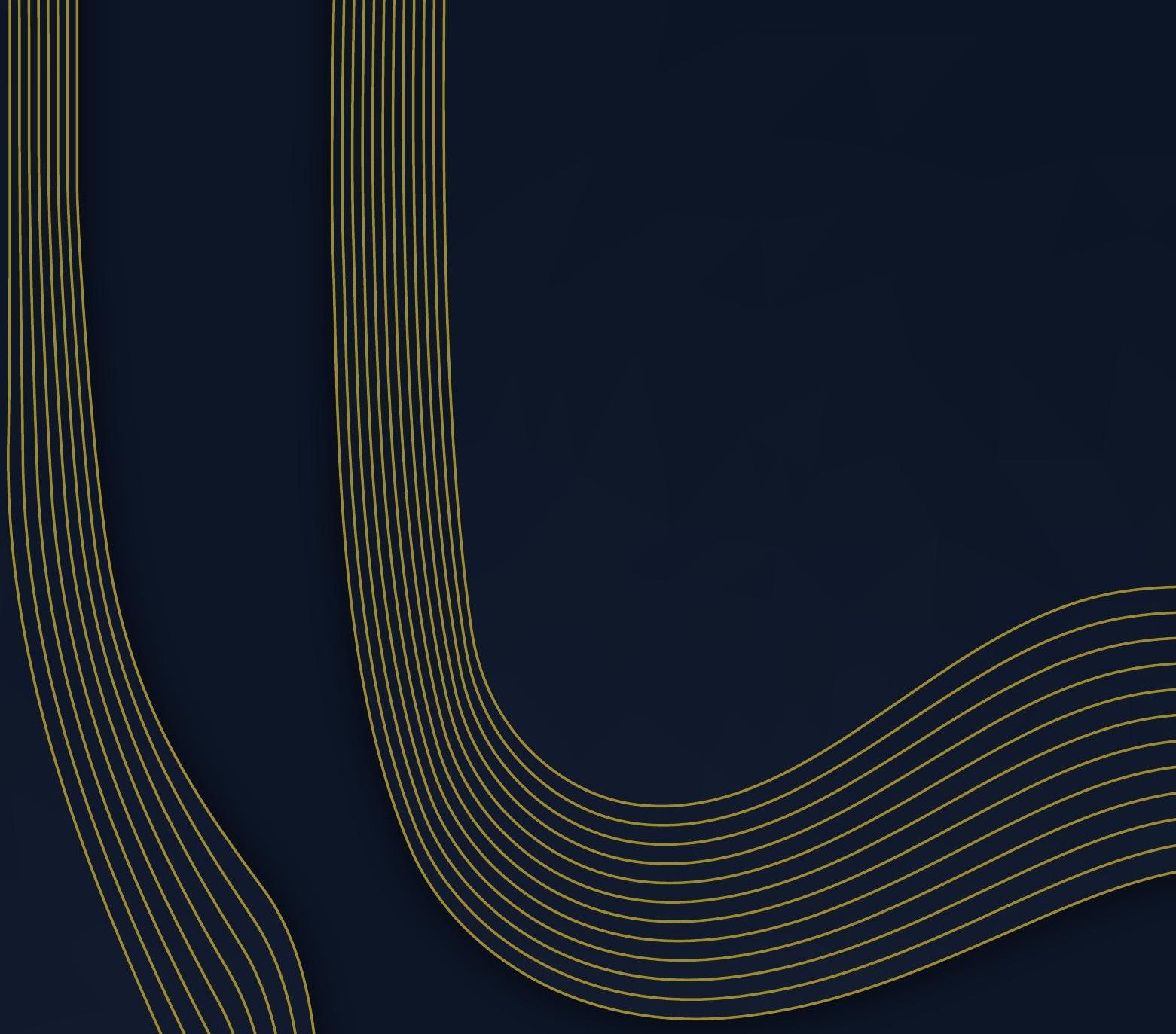

Multimodality treatment and response assessment in esophageal cancer

A L I C A B ORGGREVE 
Multimodality treatment and response assessment in esophageal cancer

Alicia Sabine Borggreve 
Multimodality treatment and response assessment in esophageal cancer

PhD thesis, Utrecht University, the Netherlands

(C) Alicia Sabine Borggreve, Utrecht, 2020. All rights reserved. No part of this thesis may be reproduced or transmitted in any form or by any means without prior written permission from the author. The copyright of the papers that have been published or have been accepted for publication has been transferred to the respective journals.

Publication of this thesis van financially supported by the Department of Radiation Oncology of the University Medical Center Utrecht, UMC Utrecht Cancer Center, Sectra Benelux, Applied Medical, the Netherlands Comprehensive Cancer Organization (IKNL), RVC Medical IT, Servier Nederland Farma and Chipsoft.

Cover \& lay-out: Wendy Schoneveld \|| www.wenziD.nl

Printed by: Gildeprint, Enschede, the Netherlands

ISBN: $\quad$ 978-94-6402-085-4 


\title{
Multimodality treatment and response assessment in esophageal cancer
}

\author{
Multimodale behandeling en evaluatie van het effect van behandeling \\ in patiënten met slokdarmkanker \\ (met een samenvatting in het Nederlands)
}

\section{Proefschrift}

ter verkrijging van de graad van doctor aan de Universiteit Utrecht op gezag van de rector magnificus, prof. dr. H.R.B.M. Kummeling, ingevolge het besluit van het college voor promoties in het openbaar te verdedigen op donderdag 12 november 2020 des middags te 2.30 uur

door

Alicia Sabine Borggreve

geboren op 8 maart 1992

te Venray 
Promotoren: Prof. dr. C.H.J. Terhaard Prof. dr. R. van Hillegersberg

Prof. dr. J.P. Ruurda

Copromotor: Dr. P.S.N. van Rossum 



\section{Contents}

Chapter 1 General introduction and thesis outline 9

\section{Part I. Neoadjuvant chemoradiotherapy}

Chapter 2 The impact of neoadjuvant chemoradiotherapy and related adverse events on short-term quality of life in esophageal cancer patients Submitted

Chapter 3 Quantification of variations in intra-fraction motion of esophageal tumors over the course of neoadjuvant chemoradiotherapy based on cine-MRI Phys Med Biol. 2018

Chapter 4 Radiation dose and pathological response in oesophageal cancer 55 patients treated with neoadjuvant chemoradiotherapy followed by surgery: a multi-institutional analysis

Acta Oncol. 2019

\section{Part II. Treatment response assessment}

Chapter 5 Preoperative prediction of pathologic response to neoadjuvant chemoradiotherapy in patients with esophageal cancer using ${ }^{18} \mathrm{~F}-\mathrm{FDG}$ PET/CT and DW-MRI: a prospective multicenter study Int J Radiat Oncol Biol Phys. 2020

Chapter 6 Optimal timing for prediction of pathologic complete response to 103 neoadjuvant chemoradiotherapy with diffusion-weighted MRI in patients with esophageal cancer

Eur Radiol. 2019

Chapter 7 Preoperative image-guided identification of response to neoadjuvant chemoradiotherapy in esophageal cancer: a multicenter observational study (PRIDE study) BMC Cancer. 2018

Chapter 8 Patient perspectives on repeated MRI and PET/CT examinations during neoadjuvant treatment of esophageal cancer Br J Radiol. 2018 


\section{Part III. Surgical treatment}

Chapter 9 Surgical treatment of esophageal cancer in the era of multimodality 161 management

Ann N Y Acad Sci. 2018

Chapter 10 Frequency of surgical resection after starting neoadjuvant chemoradiotherapy in patients with esophageal cancer:

a population-based cohort study

Eur J Surg Oncol. 2019

Chapter 11 Generalized atherosclerosis on a pre-operative CT scan is predictive for anastomotic leakage after esophagectomy

Eur J Surg Oncol. 2018

Chapter 12 Safety and feasibility of minimally invasive surgical interventions for esophageal and gastric cancer in the acute setting: a nationwide cohort study

Submitted

\section{Part IV. Summary and general discussion}

Chapter 13 Summary

Chapter 14 General discussion and future perspectives

\section{Appendices}

Summary in Dutch (Nederlandse samenvatting)

Authors and affiliations

Review committee

List of publications

Acknowledgements (Dankwoord)

Curriculum Vitae 


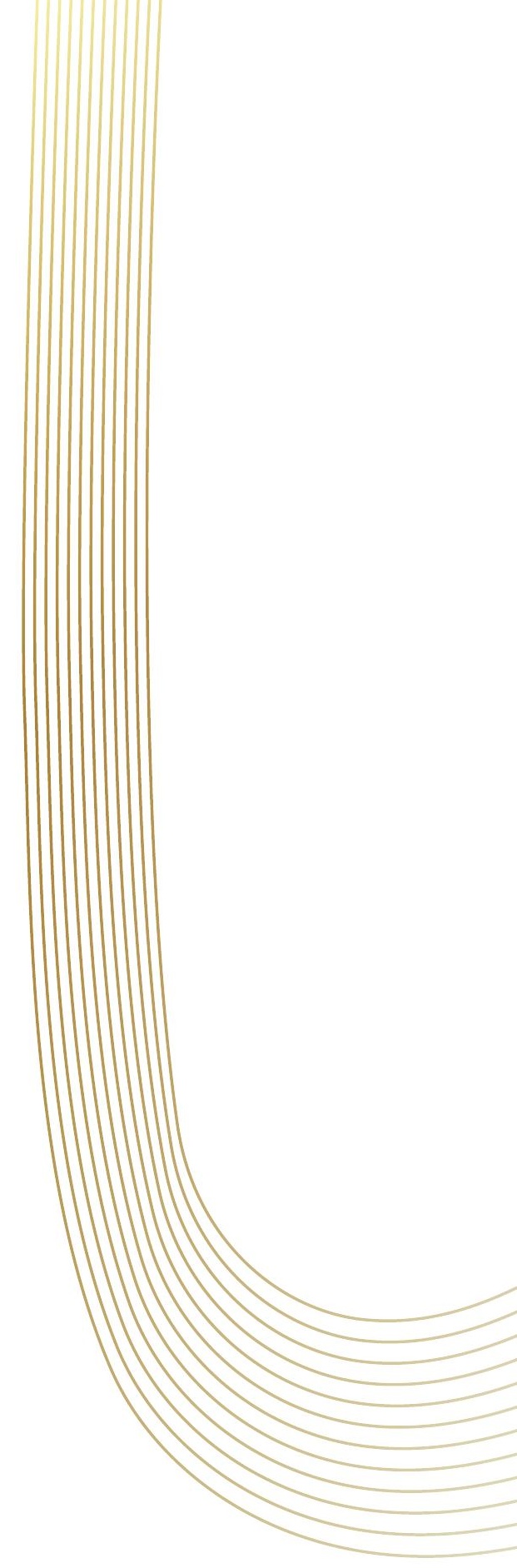


Chapter 1

General introduction and thesis outline 


\section{Esophageal cancer}

Esophageal cancer is the seventh most common type of cancer and the sixth leading cause of cancer-related deaths worldwide, with over half a million new cases and deaths yearly. ${ }^{1}$ At time of diagnosis, at least half of patients already suffer from locally unresectable or metastatic disease, leaving a minority eligible for treatment with curative intent. ${ }^{2}$ Although primary surgical resection of the esophagus with en-bloc lymphadenectomy has long been the standard for treatment with curative intent of locally advanced esophageal cancer (i.e., cT1N+M0 or cT2-4aN0-3M0), the addition of neoadjuvant therapy (i.e. neoadjuvant chemoradiotherapy or perioperative chemotherapy) has increased 5-year survival rates to 40-50\%. ${ }^{3-7}$ As clear evidence for clinical superiority of either neoadjuvant chemoradiotherapy or perioperative chemotherapy is lacking, considerable variation exists regarding the exact regimens that are applied worldwide. Nonetheless, the global consensus is that multimodality treatment, which implies neoadjuvant chemoradiotherapy or perioperative chemotherapy in combination with esophageal resection, should be the standard of care for locally advanced esophageal cancer. ${ }^{8}$

While the implementation of neoadjuvant chemoradiotherapy and perioperative chemotherapy has improved survival for patients undergoing curative treatment for esophageal cancer, all therapies carry their own efficacy and toxicity profile. As these may depend on specific patient characteristics and tumor biology, it would be beneficial to move towards tailor-made strategies for the individual patient. As such, patients who do not benefit from neoadjuvant therapy could go directly to surgery - avoiding additional toxicity -, whereas patients without residual cancer after neoadjuvant therapy (i.e., complete responders) may follow an active surveillance policy. In this light, the research presented in this thesis aims at improvements in neoadjuvant chemoradiotherapy (part I), treatment response assessment (part II) and surgical treatment (part III) in patients with esophageal cancer.

\section{Multimodality treatment}

As previously described, multimodality treatment has become standard of care for patients with locally advanced esophageal cancer. Just after the turn of the last century, the MAGIC study $^{6}$ and French FNCLCC/FFCD 9703 study $^{9}$ were the first to report an overall survival benefit for perioperative chemotherapy in patients with lower esophageal, gastroesophageal junction and gastric adenocarcinoma. Subsequently, the Dutch randomized controlled CROSS trial combined chemotherapy with 41.4 Gy radiation therapy and demonstrated a $14 \%$ increase in 5-year overall survival for patients with either squamous cell carcinoma or adenocarcinoma treated with neoadjuvant chemoradiotherapy followed by surgery 
compared to surgery alone., ${ }^{5,10}$ These results led to the widespread application of neoadjuvant chemoradiotherapy for locally advanced esophageal cancer in mainland Europe. However, considerable heterogeneity remains in neoadjuvant chemoradiotherapy regimens applied in worldwide clinical practice, with radiation dosages ranging from 35.0 to $50.4 \mathrm{~Gy}$, accompanied by various chemotherapy regimens., ${ }^{5,11-14}$

While determining the optimal treatment, it is of interest to optimize response rates while balancing the toxicities associated with neoadjuvant therapy. ${ }^{5,15,16}$ Treatment intensification with higher neoadjuvant radiation dosages might increase pathological response rates, but could also cause more adverse events. These adverse events might negatively influence a patient's quality of life, and might also prevent patients from proceeding to surgery. Toxic effects of neoadjuvant chemoradiotherapy might be reduced by better visualization of the target volume during administration of radiotherapy. Due to the presence of positioning uncertainties and respiratory motion, substantial margins are currently applied for radiotherapy. However, smaller margins might result in a reduced radiation dose to the surrounding healthy tissues, and could thereby reduce short- and long-term complications. ${ }^{17}$ Also, smaller dose plans might enable increased dosage to the tumor, while maintaining the dose constraints for healthy tissues that are currently used.

For the abovementioned reasoning, part I of this thesis will focus on optimizing response rates of neoadjuvant chemoradiotherapy while balancing the toxicities, as well as providing insight in the consequences of toxicities of neoadjuvant chemoradiotherapy for the patient.

\section{Treatment response assessment}

The improvement in survival after multimodality therapy is largely dependent on tumor response to neoadjuvant chemoradiotherapy, which can be assessed by histological examination of the resected esophagus. Nearly one third of all esophageal cancer patients (29\%) who underwent neoadjuvant chemoradiotherapy have no residual viable tumor cells at the primary tumor site upon histopathological evaluation of the resection specimen, referred to as pathologic complete response. ${ }^{5}$ Patients with a pathologic complete response have a favorable prognosis compared to other subgroups, with a lower risk of developing a locoregional recurrence and an increased survival. ${ }^{10,18-21}$ It has been argued that in patients who achieve a pathologic complete response, surgery may be omitted without substantially reducing survival. In fact, as an esophagectomy is associated with substantial morbidity, mortality (up to 3-5\%) and impaired quality of life ${ }^{22-26}$, it can be speculated that surgery may only have a detrimental effect on these patients. Consequently, proper identification of pathologic complete responders prior to surgery could yield an organ-preserving regimen avoiding esophagectomy and its postoperative complications. 
Conversely, patients with a poor response to neoadjuvant chemoradiotherapy are likely to benefit less or not at all from neoadjuvant chemoradiotherapy, but are exposed to its sideeffects. A reliable identification of poor responders during neoadjuvant chemoradiotherapy may thus also be beneficial, as ineffective therapy could be stopped or alternative treatment strategies could be explored.

Several studies have been initiated to preoperatively evaluate tumor response to neoadjuvant chemoradiotherapy in order to individualize treatment strategies. Diagnostic strategies such as endoscopic biopsies and/or endoscopic ultrasonography (EUS), ${ }^{27}{ }^{18} \mathrm{~F}$-fluorodeoxyglucose positron emission tomography with integrated computed tomography $\left({ }^{18} \mathrm{~F}-\mathrm{FDG} \mathrm{PET} /\right.$ $\mathrm{CT}),{ }^{28,29}$ diffusion weighted (DW) $)^{30-33}$ and dynamic contrast-enhanced (DCE) $)^{34}$ magnetic resonance imaging (MRI) have demonstrated their potential for response prediction, but do not yet individually fulfill the requirements to justify changes in treatment decisionmaking. As such, studies researching the performance of a multimodality imaging approach for response assessment are the main focus of this thesis and are presented in part II.

\section{Surgical treatment}

Following neoadjuvant chemoradiotherapy, esophagectomy with lymphadenectomy is recommended for all patients who did not progress during neoadjuvant therapy and are considered fit for surgery. ${ }^{4}$ Esophagectomy is a highly complex surgical procedure that is associated with higher local control rates compared to chemoradiotherapy alone, at the cost of relatively high morbidity rates. ${ }^{3}$ As such, accurate patient selection for surgery is necessary and does not only depend on tumor and treatment response characteristics, but also on assessment of comorbidities, a patients' nutritional status and cardiopulmonary function. A substantial percentage of patients diagnosed with esophageal cancer has a history of hypertension, congestive heart failure, smoking, and diabetes mellitus. ${ }^{24}$ These comorbidities are well known risk factors for several postoperative complications and might also influence whether a patient proceeds to surgery after neoadjuvant therapy.

Next to patient-related risk factors, improved diagnostic procedures may further facilitate improved patient selection. Since all patients undergo extensive preoperative staging, obtaining objective information on for instance a patient's vascular status does not require any additional tests or imaging. With these extensive possibilities, a holistic view of the patient can be obtained, providing insight in the risk of postoperative complications. Preoperative identification of patients at higher surgical risk may provide opportunities to modify these risk factors or more fully optimize patients' conditions before surgery.

Part III of this thesis will address the surgical treatment of esophageal cancer patients in the era of multimodality management, with a focus on preoperative patient selection. 


\section{Research objectives per chapter}

\section{Part I. Neoadjuvant chemoradiotherapy}

Chapter 2 To describe the short-term impact of neoadjuvant chemoradiotherapy on health-related quality of life before esophagectomy in esophageal cancer patients, and to study the impact of adverse events during neoadjuvant chemoradiotherapy on short-term health-related quality of life.

Chapter 3 To noninvasively quantify variation in intra-fraction motion of esophageal tumors over the course of neoadjuvant chemoradiotherapy using $2 \mathrm{D}$ cineMRI series.

Chapter 4 To explore whether a higher neoadjuvant radiation dose could increase pathologic tumor response rates in esophageal cancer patients.

\section{Part II. Treatment response assessment}

Chapter 5 To evaluate the individual and combined value of repeated ${ }^{18} \mathrm{~F}-\mathrm{FDG}$ PET/ CT and DW-MRI to predict pathologic response to neoadjuvant chemoradiotherapy in esophageal cancer patients.

Chapter 6 To establish the optimal timing of DW-MRI scanning during neoadjuvant chemoradiotherapy for the prediction of pathologic complete response in patients with esophageal cancer.

Chapter 7 To develop a model that predicts the probability of pathologic complete response to neoadjuvant chemoradiotherapy in esophageal cancer, based on DW-MRI, DCE-MRI and ${ }^{18} \mathrm{~F}-\mathrm{FDG}$ PET/CT.

Chapter 8 To assess the experienced burden associated with repeated MRI and PET/ CT examinations during neoadjuvant treatment for esophageal cancer from the perspective of the patient.

\section{Part III. Preoperative patient selection}

Chapter 9 To describe the current and future perspectives in the surgical treatment of patients with esophageal cancer in the context of available literature.

Chapter 10 To describe the patients with esophageal cancer who do not proceed to surgical resection after starting neoadjuvant chemoradiotherapy.

Chapter 11 To determine whether the presence of generalized atherosclerotic calcification as determined on routine CT images is associated with anastomotic leakage after esophagectomy for cancer.

Chapter 12 To evaluate whether minimally invasive esophagectomy and gastrectomy can be safely applied in the acute setting. 


\section{References}

1. GLOBOCAN Cancer Statistics 2018. http://gco.iarc.fr/.

2. Siegel RL, Miller KD, Jemal A. Cancer statistics, 2018. CA Cancer J Clin. 2018;68(1):7-30.

3. Lagergren J, Smyth E, Cunningham D, Lagergren P. Oesophageal cancer. Lancet. 2017;390(10110):23832396.

4. Lordick F, Mariette C, Haustermans K, Obermannová R, Arnold D. Oesophageal cancer: ESMO Clinical Practice Guidelines for diagnosis, treatment and follow-up. Ann Oncol. 2016;27(suppl_5):v50-v57.

5. van Hagen P, Hulshof MCCM, van Lanschot JJB, et al. Preoperative Chemoradiotherapy for Esophageal or Junctional Cancer. N Engl J Med. 2012;366(22):2074-2084.

6. Cunningham D, Allum WWH, Stenning SSP, et al. Perioperative chemotherapy versus surgery alone for resectable gastroesophageal cancer. N Engl J Med. 2006;355(1):11-20.

7. Kelsen DP, Ginsberg R, Pajak TF, et al. Chemotherapy Followed by Surgery Compared with Surgery Alone for Localized Esophageal Cancer. N Engl J Med. 1998;339(27):1979-1984.

8. Al-Batran S-E, Lorenzen S, Lilly E, Lorenzen S. Management of Locally Advanced Gastroesophageal Cancer Still a Multidisciplinary Global Challenge? Hematol Oncol Clin N Am. 2017;31:441-452.

9. Ychou M, Boige V, Pignon J, et al. Perioperative chemotherapy compared with surgery alone for resectable gastroesophageal adenocarcinoma: an FNCLCC and FFCD multicenter phase III trial. J Clin Oncol. 2011;29(13):1715.

10. Shapiro J, Lanschot JJB Van, Hulshof MCCM, Hagen P Van, Henegouwen MIVB, Wijnhoven BPL. Neoadjuvant chemoradiotherapy plus surgery versus surgery alone for oesophageal or junctional cancer (CROSS): long-term results of a randomised controlled trial. Lancet Oncol. 2015;16(9):1090-1098.

11. Walsh TN, Noonan N, Hollywood D, Kelly A, Keeling N, Hennessy TPJ. A Comparison of Multimodal Therapy and Surgery for Esophageal Adenocarcinoma. N Engl J Med. 1996;335(7):462-467.

12. Burmeister BH, Smithers BM, Gebski V, et al. Surgery alone versus chemoradiotherapy followed by surgery for resectable cancer of the oesophagus: a randomised controlled phase III trial. Lancet Oncol. 2005;6(9):659-668.

13. Tepper J, Krasna MJ, Niedzwiecki D, et al. Phase III trial of trimodality therapy with cisplatin, fluorouracil, radiotherapy, and surgery compared with surgery alone for esophageal cancer: CALGB 9781. J Clin Oncol. 2008;26(7):1086-1092.

14. Sjoquist KM, Burmeister BH, Smithers BM, et al. Survival after neoadjuvant chemotherapy or chemoradiotherapy for resectable oesophageal carcinoma: an updated meta-analysis. Lancet Oncol. 2011;12(7):681-692.

15. Lin SH, Wang J, Allen PK, et al. A nomogram that predicts pathologic complete response to neoadjuvant chemoradiation also predicts survival outcomes after definitive chemoradiation for esophageal cancer. $J$ Gastrointest Oncol. 2015;6(1):45-52.

16. Meredith KL, Weber JM, Turaga KK, et al. Pathologic response after neoadjuvant therapy is the major determinant of survival in patients with esophageal cancer. Ann Surg Oncol. 2010;17(4):1159-1167.

17. Johnson-Hart CN, Price GJ, Faivre-Finn C, Aznar MC, van Herk M. Residual Setup Errors Towards the Heart After Image Guidance Linked With Poorer Survival in Lung Cancer Patients: Do We Need Stricter IGRT Protocols? Int J Radiat Oncol. 2018;102(2):434-442.

18. Oppedijk V, van der Gaast A, van Lanschot JJ, et al. Patterns of recurrence after surgery alone versus preoperative chemoradiotherapy and surgery in the CROSS trials. J Clin Oncol. 2014;32(5):385-391.

19. Alnaji RM, Du W, Gabriel E, et al. Pathologic Complete Response Is an Independent Predictor of Improved Survival Following Neoadjuvant Chemoradiation for Esophageal Adenocarcinoma. J Gastrointest Surg. 2016;20(9):1541-1546.

20. Donahue JM, Nichols FC, Li Z, et al. Complete Pathologic Response After Neoadjuvant Chemoradiotherapy for Esophageal Cancer Is Associated With Enhanced Survival. Ann Thorac Surg. 2009;87(2):392-399.

21. Berger AC, Farma J, Scott WJ, et al. Complete Response to Neoadjuvant Chemoradiotherapy in Esophageal Carcinoma Is Associated With Significantly Improved Survival. J Clin Oncol. 2005;23(19):4330-4337.

22. Djärv T, Lagergren J, Blazeby JM, Lagergren P. Long-term health-related quality of life following surgery for oesophageal cancer. Br J Surg. 2008;95(9):1121-1126. 
23. Mc Cormack O, Zaborowski A, King S, et al. New-onset Atrial Fibrillation Post-surgery for Esophageal and Junctional Cancer Incidence, Management, and Impact on Short-and Long-term Outcomes. Ann Surg. 2014;260(5):772-778.

24. Kassis ES, Kosinski AS, Ross P, Koppes KE, Donahue JM, Daniel VC. Predictors of anastomotic leak after esophagectomy: An analysis of the society of thoracic surgeons general thoracic database. Ann Thorac Surg. 2013;96(6):1919-1926.

25. Busweiler LAD, Wijnhoven BPL, van Berge Henegouwen MI, et al. Early outcomes from the Dutch Upper Gastrointestinal Cancer Audit. Br J Surg. 2016;103(13):1855-1863.

26. Schandl A, Lagergren J, Johar A, Lagergren P. Health-related quality of life 10 years after oesophageal cancer surgery. Eur J Cancer. 2016;69:43-50.

27. van Rossum PSN, Goense L, Meziani J, et al. Endoscopic biopsy and EUS for the detection of pathologic complete response after neoadjuvant chemoradiotherapy in esophageal cancer: a systematic review and meta-analysis. Gastrointest Endosc. 2016;83(5):866-879.

28. Kwee RM. Prediction of Tumor Response to Neoadjuvant Therapy in Patients with Esophageal Cancer with Use of ${ }^{18}$ F FDG PET: A Systematic Review. Radiology. 2010;254(3):707-717.

29. van Rossum PSN, Fried D V, Zhang L, et al. The Incremental Value of Subjective and Quantitative Assessment of 18F-FDG PET for the Prediction of Pathologic Complete Response to Preoperative Chemoradiotherapy in Esophageal Cancer. J Nucl Med. 2016;57(5):691-700.

30. Aoyagi T, Shuto K, Okazumi S, Shimada H, Kazama T, Matsubara H. Apparent diffusion coefficient values measured by diffusion-weighted imaging predict chemoradiotherapeutic effect for advanced esophageal cancer. Dig Surg. 2011;28(4):252-257.

31. De Cobelli F, Giganti F, Orsenigo E, et al. Apparent diffusion coefficient modifications in assessing gastrooesophageal cancer response to neoadjuvant treatment: Comparison with tumour regression grade at histology. Eur Radiol. 2013;23(8):2165-2174.

32. van Rossum PSN, van Lier ALHMW, van Vulpen M, et al. Diffusion-weighted magnetic resonance imaging for the prediction of pathologic response to neoadjuvant chemoradiotherapy in esophageal cancer. Radiother Oncol. 2015;115(2):163-170.

33. Wang L, Liu L, Han C, et al. The diffusion-weighted magnetic resonance imaging (DWI) predicts the early response of esophageal squamous cell carcinoma to concurrent chemoradiotherapy. Radiother Oncol. 2016;121:246-251.

34. Heethuis SE, van Rossum PSN, Lips IM, et al. Dynamic contrast-enhanced MRI for treatment response assessment in patients with oesophageal cancer receiving neoadjuvant chemoradiotherapy. Radiother Oncol. 2016;120(1):128-135. 



\section{PART I}

Neoadjuvant chemoradiotherapy 


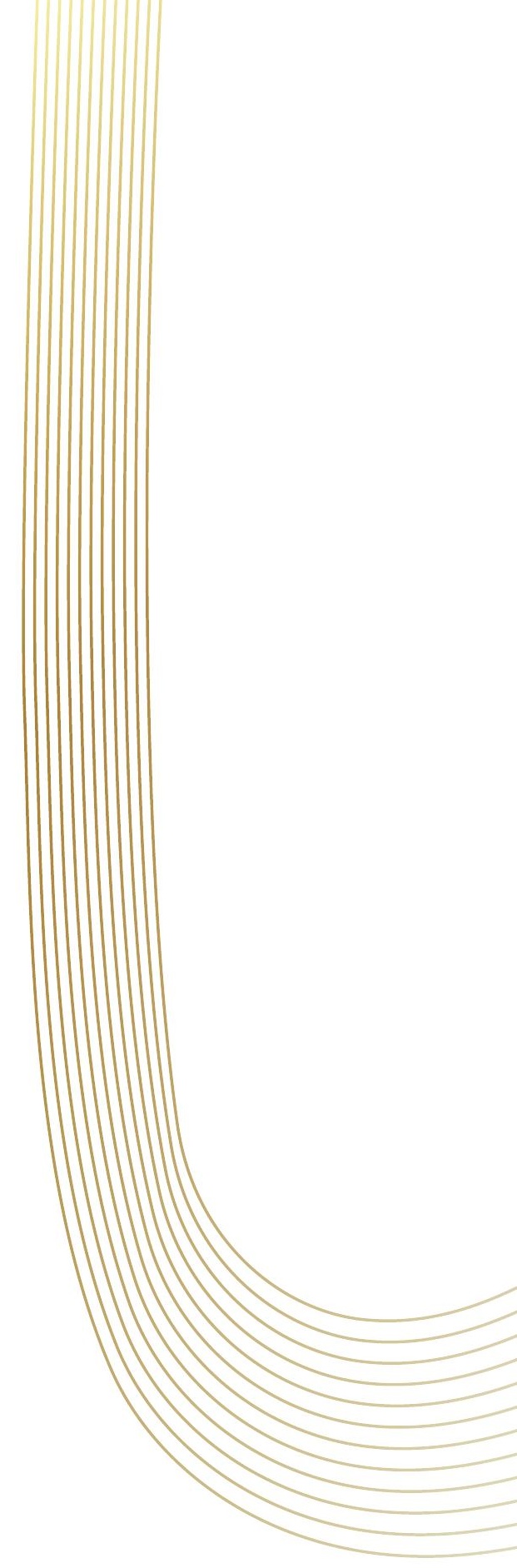


Chapter 2

The impact of neoadjuvant chemoradiotherapy and related adverse events on short-term quality of life in esophageal cancer patients

Submitted

A.S. Borggreve, P.S.N. van Rossum, J.P. Ruurda, H.M. Verkooijen, G.J. Meijer, N. Haj Mohammad, I.M. Lips, R. van Hillegersberg, S. Mook 


\begin{abstract}

\section{Background}

Insights into the impact of neoadjuvant chemoradiotherapy (nCRT) on short-term healthrelated quality of life (HRQOL) might be helpful for patients and physicians in the process of shared decision making. The aim of this study was to describe the short-term impact of nCRT on HRQOL before esophagectomy in esophageal cancer patients, and to study the impact of adverse events during nCRT on short-term HRQOL.
\end{abstract}

\title{
Methods
}

HRQOL was measured in patients with locally advanced esophageal cancer referred to the Department of Radiation Oncology before start of nCRT and 1 month after completion of nCRT, using EORTC questionnaires (QLQ-C30 and QLQ-OG25). Changes in HRQOL between baseline and follow up, were compared between patients with and without adverse events (CTCAE grade $\geq 3$ ) during nCRT with mixed model analyses.

\section{Results}

In total 115 patients returned the baseline questionnaire of whom 85 patients $(73.9 \%)$ returned the follow-up questionnaire. Adverse events during nCRT occurred in 30 patients (26.1\%). During nCRT, physical functioning declined and fatigue increased, whereas odynophagia symptoms decreased at 1 month follow-up. General HRQOL remained stable. Adverse events during nCRT had a negative effect on dysphagia, pain and taste at followup.

\section{Conclusion}

Physical functioning and fatigue deteriorated in esophageal cancer patients treated with nCRT. The occurrence of adverse events during nCRT was associated with a sharper impairment of HRQOL and esophageal cancer specific symptoms. 


\section{Introduction}

Neoadjuvant chemoradiotherapy (nCRT) followed by esophagectomy is the preferred treatment for patients with locally advanced esophageal cancer, improving overall survival compared to esophagectomy alone. ${ }^{1,2}$ In recent years, the emphasis on health-related quality of life (HRQOL) and other patient-reported outcome measures has enhanced, resulting in a more prominent role for both the quality and the quantity of life lived after cancer diagnosis. ${ }^{3}$ This is reflected in the incorporation of HRQOL as an important endpoint when evaluating treatment efficacy, which is already executed in for example the ESOPEC trial comparing nCRT to perioperative chemotherapy for esophageal cancer. ${ }^{4}$

Regarding the long-term effects of nCRT, it is known that even though patients experience deterioration in HRQOL end-points after completion of nCRT, nCRT does not affect longterm HRQOL compared to surgery alone. ${ }^{5-7}$ In contrast, severe complications after surgery have been consistently demonstrated to negatively impact HRQOL in the short and long term. ${ }^{8-11}$ However, not only surgical treatment, but also treatment with nCRT is accompanied by serious (non-hematologic) adverse events in $13 \% .^{2}$ The effect of nCRT on HRQOL has been described before ${ }^{7,12}$, but insight is lacking in how adverse events during nCRT affect short-term HRQOL. This information could be used by physicians to adequately manage patients' expectations about their HRQOL shortly after nCRT, so that patients can anticipate on these effects and take proper measurement to cope with these problems.

Therefore, the aim of the current study was to describe the short-term impact of nCRT on HRQOL before esophagectomy in esophageal cancer patients, and to study how adverse events during nCRT impact short-term HRQOL.

\section{Methods}

This single center cohort study was reviewed and approved by the institutional review board of the University Medical Center Utrecht (number 11/515), and the need for written informed consent was waived.

\section{Study population}

Consecutive patients with histologically confirmed cancer of the esophagus or gastroesophageal junction who were scheduled to undergo nCRT followed by esophagectomy between October 2011 and August 2017 at the Department of Radiation Oncology were eligible for inclusion. Due to logistic reasons, only patients referred to the department from outside our center were sent questionnaires. Exclusion criteria included not completing the baseline questionnaire, completing the baseline questionnaire after starting nCRT and starting treatment other than nCRT. 
Neoadjuvant treatment consisted of weekly intravenous administration of carboplatin area under the curve $2 /$ paclitaxel $50 \mathrm{mg} / \mathrm{m}^{2}$ for 5 weeks and concurrent radiotherapy $(41.4 \mathrm{~Gy}$ in 23 fractions of $1.8 \mathrm{~Gy}$ ), as per the CROSS regimen. ${ }^{2}$ Surgical resection was generally scheduled 6-8 weeks after neoadjuvant chemoradiotherapy according to the Dutch guidelines. $^{13}$

Data regarding patient and tumor characteristics, as well as treatment-related variables were collected from a prospectively maintained database. Serious adverse events during nCRT were defined as adverse events grade 3 or higher according to the National Cancer Institute's Common Terminology Criteria for Adverse Events (CTCAE 4.0). Discontinuation of chemotherapy due to hematologic toxicity without the need for hospitalization or other interventions, as well as dysphagia for which tube feeding was started before nCRT were not included as serious adverse events.

\section{Quality of life assessment}

Eligible patients received self-report questionnaires by postal mail with their invitation for their first appointment (baseline), and 1 month after finishing nCRT (follow-up). Patients who did not return the baseline questionnaire were not invited to participate in the followup questionnaire. At both time points, 2 validated questionnaires were sent.

Cancer-specific HRQOL was measured with the European Organization for Research and Treatment of Cancer (EORTC) Quality of Life Questionnaire - Core 30 (QLQ-C30, version 3.0). ${ }^{14}$ The structure of the QLQ-C30 has been reported in detail elsewhere and its validity and reliability has been examined in several diagnostic groups, including esophageal cancer patients. ${ }^{15,16}$ Esophageal cancer specific HRQOL was measured by the EORTC QLQOesophago-Gastric Module (QLQ-OG25). ${ }^{17}$

All scale and item scores from the QLQ-C30 and QLQ-OG25 were linearly transformed to a $0-100$ score according to the EORTC scoring manuals. ${ }^{18}$ High scores in the multi-item functional scales and the global quality of life scale indicated higher levels of function and quality of life (QLQ-C30), whereas higher scores on the symptom scales represented more symptoms (QLQ-C30, QLQ-OG25). For missing items from multi-item scales, the following was assumed as per recommendation of the developers: if at least half of the items from the scale had been answered, the missing items would have had values equal to the average of those items which were present for that patient. ${ }^{18}$ The QLQ-C30 Summary Score ${ }^{16}$ was only calculated for a respondent if all 13 scales contributing to that score were completed. In order to enhance the interpretation of the symptom scales of the QLQ-OG25, the percentage of symptomatic patients was calculated. Symptomatic refers to patients who answered 'a little', 'quite a bit', or 'very much' to any question within a scale or to a single item. Others were categorized as having no symptoms. ${ }^{19}$ 


\section{Statistical analysis}

Patient and treatment-related characteristics were presented as counts with percentages, means ( \pm standard deviation $[\mathrm{SD}]$ ) or medians (interquartile range $[\mathrm{IQR}]$ ) where appropriate. Baseline HRQOL scale and symptom scores of all questionnaires were described as means with SD.

To compare HRQOL before and after administration of nCRT, linear mixed-effects models were used to calculate mean score differences (MDs) with 95\% confidence intervals (CIs) between baseline and follow-up HRQOL scores, accounting for potential confounders. To assess the impact of occurrence of adverse events during nCRT on HRQOL scales, linear mixed-effects models were used with an adverse event-by-time interaction, accounting for potential confounders. All models included a patient-specific random intercept and were adjusted for the following confounding variables: age, sex, performance status, clinical $\mathrm{T}$ stage and clinical N stage. For the QLQ-C30 subscales, clinically relevant differences in HRQOL scores have been established before. ${ }^{20}$ For the QLQ-OG25, no cut-off values had been established before. As such, MDs of 10-20 points were considered as moderate clinical difference and $>20$ points as large clinical difference. ${ }^{21}$

Lastly, in order to visualize differences in the distribution and probability density of the HRQOL scores at baseline and follow-up, as well as between patients with adverse events and without adverse events, rotated kernel density plots were constructed.

All statistical analyses were performed using R 3.5.1 open-source software ('PROscorer', 'Ime4' and 'lmerTest' packages, www.R-project.org). In order to minimize the effect of multiple testing and type I errors, a two-sided p-value $<0.01$ was deemed significant.

\section{Results}

\section{Patients}

Between October 2011 and July 2017, 115 patients who started neoadjuvant chemoradiotherapy for esophageal cancer completed the baseline questionnaire before starting nCRT (Figure 1). The mean age was 66.9 years ( \pm SD: 7.3 years) and the majority (78.3\%) was male (Table 1$)$. Histologic tumor types mainly included adenocarcinoma (60.9\%) and squamous cell carcinoma (35.7\%). Severe adverse events during nCRT (CTCAE grade $\geq 3$ ) occurred in 30 patients $(26.1 \%$ ) (Table 2 ).

The baseline questionnaire was completed 14 days (median, IQR: 10-17 days) before starting nCRT. Follow-up questionnaires after nCRT and before surgery were available in 85 patients (73.9\%), and were completed 37 days (median, IQR: 35-43 days) after the last day of nCRT. The clinical characteristics of follow-up questionnaire responders and non-responders were similar, with the exception of the occurrence of severe adverse events $(20.0 \%$ versus $43.3 \%$ respectively, Table 1 ). 


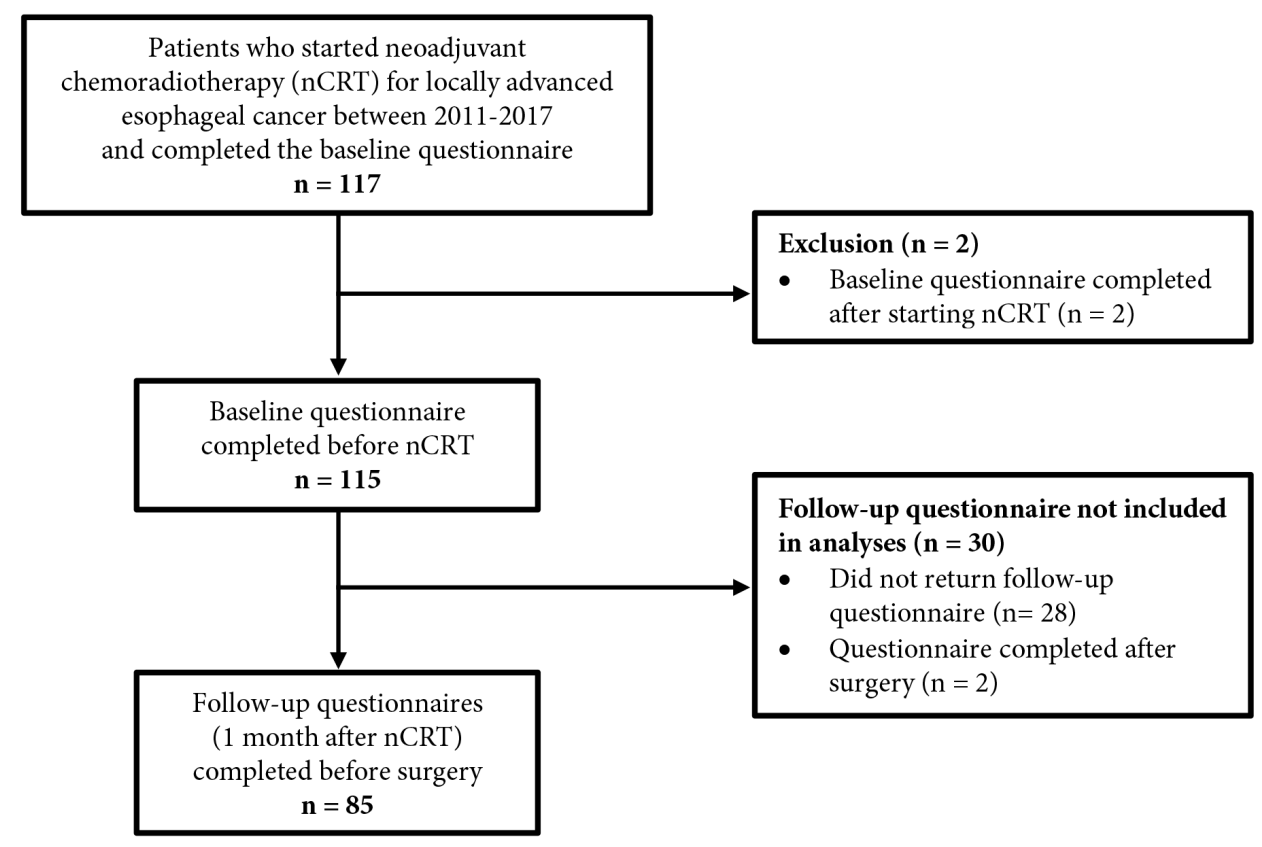

Figure 1. Flowchart

\section{General quality of life}

The HRQOL scales and items for QLQ-C30 are shown in Table 3. At baseline, global quality of life was highly skewed (Figure 2) with a mean score of $67(\mathrm{SD} \pm 20$ ). Fatigue (mean \pm SD: $30 \pm 24$ ), insomnia (mean \pm SD: $26 \pm 28$ ), pain (mean \pm SD: $22 \pm 22$ ) and loss of appetite (mean \pm SD: $21 \pm 30$ ) were the most prevalent general symptoms at baseline.

Global quality of life and general health scores were similar at baseline and 1 month after nCRT. Clinically relevant and statistically significant declines were however observed in physical functioning (MD [95\% CI]: $-8[-12,-5])$ and social functioning (MD [95\% CI]: -8 $[-13,-4])$. Furthermore, patients experienced significantly more fatigue (MD [95\% CI]: 7 $[2,11]$ ) and dyspnea (MD [95\% CI]: $10[5,14]) 1$ month after nCRT. Absolute mean scores of HRQOL items and the available number of respondents per HRQOL item are provided in Supplementary Table 2. 
Table 1. Clinical characteristics of the study population.

\begin{tabular}{|c|c|c|c|c|c|c|}
\hline \multirow[t]{2}{*}{ Characteristics } & \multicolumn{2}{|c|}{$\begin{array}{l}\text { Full cohort } \\
\qquad(\mathrm{n}=115)\end{array}$} & \multicolumn{2}{|c|}{$\begin{array}{l}\text { Baseline and } \\
\text { follow-up } \\
\text { questionnaire } \\
(\mathbf{n}=\mathbf{8 5})\end{array}$} & \multicolumn{2}{|c|}{$\begin{array}{c}\text { Baseline } \\
\text { questionnaire } \\
\text { only } \\
(\mathbf{n}=\mathbf{3 0})\end{array}$} \\
\hline & $\mathrm{n}$ & $(\%)$ & $\mathrm{n}$ & $(\%)$ & $\mathrm{n}$ & $(\%)$ \\
\hline $\begin{array}{l}\text { Age at diagnosis, in years } \\
(\text { mean } \pm S D)\end{array}$ & \multicolumn{2}{|c|}{$66.9 \pm 7.3$} & \multicolumn{2}{|c|}{$67.3 \pm 7.1$} & \multicolumn{2}{|c|}{$65.9 \pm 9.7$} \\
\hline \multicolumn{7}{|l|}{ Sex } \\
\hline Male & 90 & $78.3 \%$ & 71 & $83.5 \%$ & 19 & $63.3 \%$ \\
\hline Female & 25 & $21.7 \%$ & 14 & $16.5 \%$ & 11 & $36.7 \%$ \\
\hline \multicolumn{7}{|l|}{ WHO performance status } \\
\hline 0 & 53 & $46.1 \%$ & 43 & $50.6 \%$ & 10 & $33.3 \%$ \\
\hline 1 & 59 & $51.3 \%$ & 41 & $48.2 \%$ & 18 & $60.0 \%$ \\
\hline 2 & 3 & $2.6 \%$ & 1 & $1.2 \%$ & 2 & $6.7 \%$ \\
\hline \multicolumn{7}{|l|}{ Clinical T stage $^{\S}$} \\
\hline cT1 1 & 2 & $1.7 \%$ & 1 & $1.2 \%$ & 1 & $3.3 \%$ \\
\hline cT2 & 17 & $14.8 \%$ & 14 & $16.5 \%$ & 3 & $10.0 \%$ \\
\hline cT3 & 90 & $78.3 \%$ & 67 & $78.8 \%$ & 23 & $76.7 \%$ \\
\hline cT4 & 6 & $5.2 \%$ & 3 & $3.5 \%$ & 3 & $10.0 \%$ \\
\hline \multicolumn{7}{|l|}{ Clinical N stage $^{\mathfrak{s}}$} \\
\hline cN0 & 32 & $27.8 \%$ & 24 & $28.2 \%$ & 8 & $26.7 \%$ \\
\hline $\mathrm{cN} 1$ & 60 & $52.2 \%$ & 42 & $49.4 \%$ & 18 & $60.0 \%$ \\
\hline $\mathrm{cN} 2$ & 19 & $16.5 \%$ & 15 & $17.6 \%$ & 4 & $13.3 \%$ \\
\hline $\mathrm{cN} 3$ & 4 & $3.5 \%$ & 4 & $4.7 \%$ & 0 & $0.0 \%$ \\
\hline \multicolumn{7}{|l|}{ Tumor location } \\
\hline Upper esophagus & 4 & $3.5 \%$ & 4 & $4.7 \%$ & 0 & $0.0 \%$ \\
\hline Middle esophagus & 22 & $19.1 \%$ & 14 & $16.5 \%$ & 8 & $26.7 \%$ \\
\hline Distal esophagus/gastroesophageal junction & 89 & $77.4 \%$ & 67 & $78.8 \%$ & 22 & $73.3 \%$ \\
\hline \multicolumn{7}{|l|}{ Histologic tumor type } \\
\hline Adenocarcinoma & 70 & $60.9 \%$ & 56 & $65.9 \%$ & 14 & $46.7 \%$ \\
\hline Squamous cell carcinoma & 41 & $35.7 \%$ & 25 & $29.4 \%$ & 16 & $52.3 \%$ \\
\hline Other & 4 & $3.5 \%$ & 4 & $4.7 \%$ & 0 & $0.0 \%$ \\
\hline $\begin{array}{l}\text { Adverse events of CTCAE grade } \geq 3 \text { during } \\
\text { neoadjuvant chemoradiotherapy }\end{array}$ & 30 & $26.0 \%$ & 17 & $20.0 \%$ & 13 & $43.3 \%$ \\
\hline $\begin{array}{l}\text { Days between baseline questionnaire and start } \\
\text { nCRT (median, IQR) }\end{array}$ & \multicolumn{2}{|c|}{$14(10,17)$} & \multicolumn{2}{|c|}{$14(11,17)$} & \multicolumn{2}{|c|}{$14(5,15)$} \\
\hline $\begin{array}{l}\text { Days between end nCRT and follow-up } \\
\text { questionnaire (median, IQR) }\end{array}$ & \multicolumn{2}{|c|}{$37(35,43)$} & \multicolumn{2}{|c|}{$37(35,43)$} & \multicolumn{2}{|c|}{ NA } \\
\hline
\end{tabular}

CTCAE Common Terminology Criteria of Adverse Events (version 4.0); IQR interquartile range; NA not applicable; $S D$ standard deviation; WHO World Health Organization

${ }^{s}$ Clinical $\mathrm{T}$ and $\mathrm{N}$ stage are based on AJCC TNM $7^{\text {th }}$ edition.

' Both patients had a cT1N1 stage. 
Table 2. Adverse events of CTCAE grade $\geq 3$ during neoadjuvant chemoradiotherapy for esophageal cancer in the study population (baseline scores excluded).

\begin{tabular}{lcc}
\hline Adverse events & \multicolumn{2}{c}{ Total (n=115) } \\
\cline { 2 - 3 } & $\mathrm{n}$ & $\%$ \\
\hline All & 30 & $26.1 \%$ \\
Dysphagia & 13 & $11.3 \%$ \\
Leukopenia & 4 & $3.5 \%$ \\
Thromboembolic event & 3 & $2.6 \%$ \\
Nausea & 3 & $2.6 \%$ \\
Vomiting & 2 & $1.7 \%$ \\
Anorexia & 2 & $1.7 \%$ \\
Febrile neutropenia & 2 & $1.7 \%$ \\
Fatigue & 1 & $0.9 \%$ \\
Esophageal perforation & 1 & $0.9 \%$ \\
Dyspnea & 1 & $0.9 \%$ \\
Allergic reaction & 1 & $0.9 \%$ \\
\hline
\end{tabular}

\section{Esophageal-specific symptoms}

At baseline, the most pronounced esophageal cancer-specific symptom as measured by the QLQ-OG25 questionnaire was anxiety (99.1\%, mean \pm SD: $46 \pm 19)$, followed by eating restrictions (87.0\%, mean \pm SD: $32 \pm 23$ ), odynophagia (85.8\%, mean \pm SD: $29 \pm 23$ ) and dysphagia (85.2\%, mean \pm SD: $24 \pm 20$ ) (Table 4, Figure 2). After nCRT, the most pronounced symptom remained anxiety (96.5\%), followed by eating restrictions $(65.1 \%)$ and trouble with coughing (63.9\%). Clinically relevant and statistically significant effects of nCRT were observed in decreased odynophagia and decreased problems with eating in front of others (MD [95\% CI]: $-13[-18,-9]$ and $-10[-15,-5]$, respectively).

\section{Adverse events during nCRT and short-term quality of life}

Patients who experienced a severe adverse event (CTCAE grade $\geq 3$ ) had significantly more pain and discomfort, more advanced dysphagia scores and reported more trouble with taste after nCRT, compared to patients who did not experience adverse events (Table 4, Figure 3). Global quality of life after nCRT as measured by the QLQ-C30 and other symptom scales did not differ between patients with and without adverse events during nCRT (Table 3 and 4, Figure 3). 
Table 3. Health-related quality of life (HRQOL)-scores of esophageal cancer patients before starting neoadjuvant chemoradiotherapy (nCRT) (baseline) and adjusted mean score differences 1 month after nCRT (follow-up), as well as at follow-up in patients who experienced adverse events during nCRT compared to patients without adverse events according to the EORTC QLQ-C30 questionnaire.

\begin{tabular}{|c|c|c|c|c|}
\hline \multirow[t]{2}{*}{$\begin{array}{l}\text { Quality of life dimension } \\
\text { (QLQ-C30) }\end{array}$} & \multicolumn{2}{|c|}{$\begin{array}{l}\text { Baseline } \\
(n=115)\end{array}$} & \multirow{2}{*}{$\begin{array}{l}\begin{array}{l}\text { Follow-up } \\
(\mathrm{n}=85)\end{array} \\
\begin{array}{l}\text { Mean difference } \\
\text { compared to baseline } \\
(95 \% \mathrm{CI})\end{array}\end{array}$} & \multirow{2}{*}{$\begin{array}{l}\text { Adverse events during nCRT } \\
\text { Mean difference in follow-up } \\
\text { HRQOL scores compared to } \\
\text { patients without adverse } \\
\text { events (95\% CI) }\end{array}$} \\
\hline & $\begin{array}{l}\text { Mean } \\
\text { score }\end{array}$ & SD & & \\
\hline Global quality of life $\mathrm{s}^{\S}$ & 67 & 20 & $2(-2,6)$ & $-5(-15,5)$ \\
\hline \multicolumn{5}{|l|}{ Functional scales ${ }^{\S}$} \\
\hline Physical functioning & 85 & 17 & $-8(-12,-5)^{*}$ & $-7(-16,2)$ \\
\hline Role functioning & 77 & 27 & $-6(-12,-1)$ & $-10(-25,4)$ \\
\hline Emotional functioning & 70 & 20 & $5(1,9)$ & $-10(-21,0)$ \\
\hline Cognitive functioning & 84 & 20 & $-1(-5,2)$ & $-5(-15,4)$ \\
\hline Social functioning & 83 & 23 & $-8(-13,-4)^{\star}$ & $-14(-26,-2)$ \\
\hline \multicolumn{5}{|l|}{ Symptom scales' } \\
\hline Fatigue & 30 & 24 & $7(2,11)^{*}$ & $13(1,24)$ \\
\hline Nausea and vomiting & 11 & 17 & $-1(-6,4)$ & $4(-5,14)$ \\
\hline Pain & 22 & 22 & $-1(-6,5)$ & $8(-4,21)$ \\
\hline \multicolumn{5}{|l|}{ Single-item symptom scores } \\
\hline Dyspnea & 13 & 20 & $10(5,14)^{*}$ & $1(-11,12)$ \\
\hline Insomnia & 26 & 28 & $-2(-7,3)$ & $5(-9,19)$ \\
\hline Loss of appetite & 21 & 30 & $0(-8,7)$ & $9(-7,24)$ \\
\hline Constipation & 15 & 26 & $-4(-9,2)$ & $2(-11,14)$ \\
\hline Diarrhea & 5 & 15 & $4(-1,8)$ & $-3(-13,6)$ \\
\hline Financial difficulties & 9 & 25 & $1(-1,4)$ & $-3(-14,8)$ \\
\hline QLQ-C30 Summary Score ${ }^{\S ¥}$ & 81 & 12 & $-2(-5,0)$ & $-6(-13,1)$ \\
\hline
\end{tabular}

CI confidence interval, $n C R T$ neoadjuvant chemoradiotherapy, SD standard deviation

Values in bold are both clinically relevant $\left(\right.$ Cocks et al..$^{20}$ ) and statistically significant $\left(^{\star}\right)$.

$\checkmark$ Score range 0-100. Higher scores represent a better quality of life or higher level of functioning.

' Score range 0-100. Higher scores represent more severe symptoms or problems.

* Statistically significant differences $(\mathrm{p}<0.01$ ) based on multivariable linear mixed effect models including a random intercept per patient and adjusted for age, sex, performance status, clinical T stage and clinical $\mathrm{N}$ stage.

Mean of all scores except for global quality of life and financial difficulties. ${ }^{16}$ 

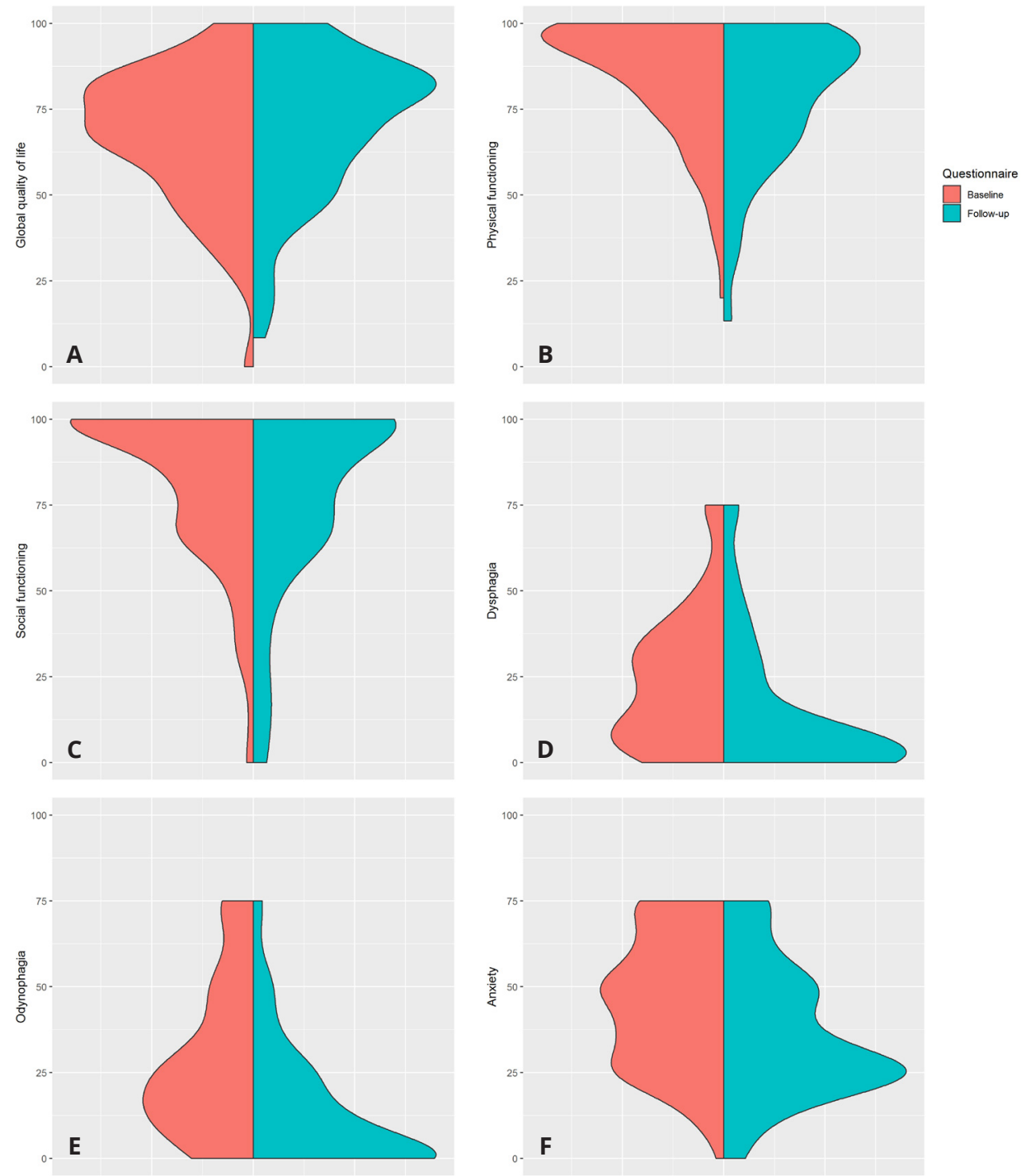

Figure 2. Rotated kernel density plots of global quality of life $(A)$, physical functioning $(B)$, social functioning $(C)$ scales (according to the EORTC QLQ-C30 questionnaire) and dysphagia $(D)$, odynophagia $(E)$ and anxiety $(C)$ symptoms (according to the EORTC QLQ-OG25 questionnaire) at baseline (before neoadjuvant chemoradiotherapy, red) and follow-up (1 month after neoadjuvant chemoradiotherapy, blue). 


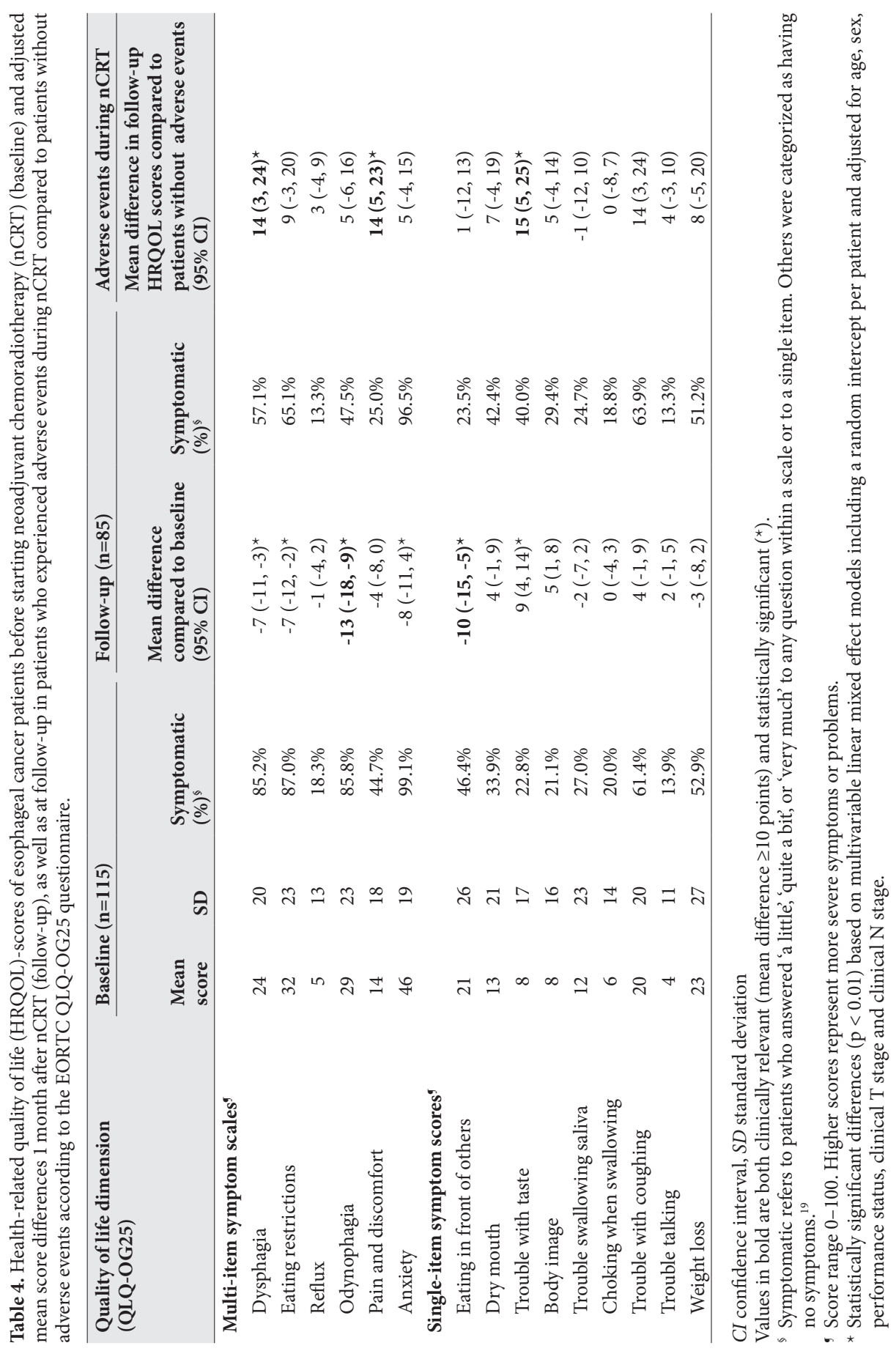



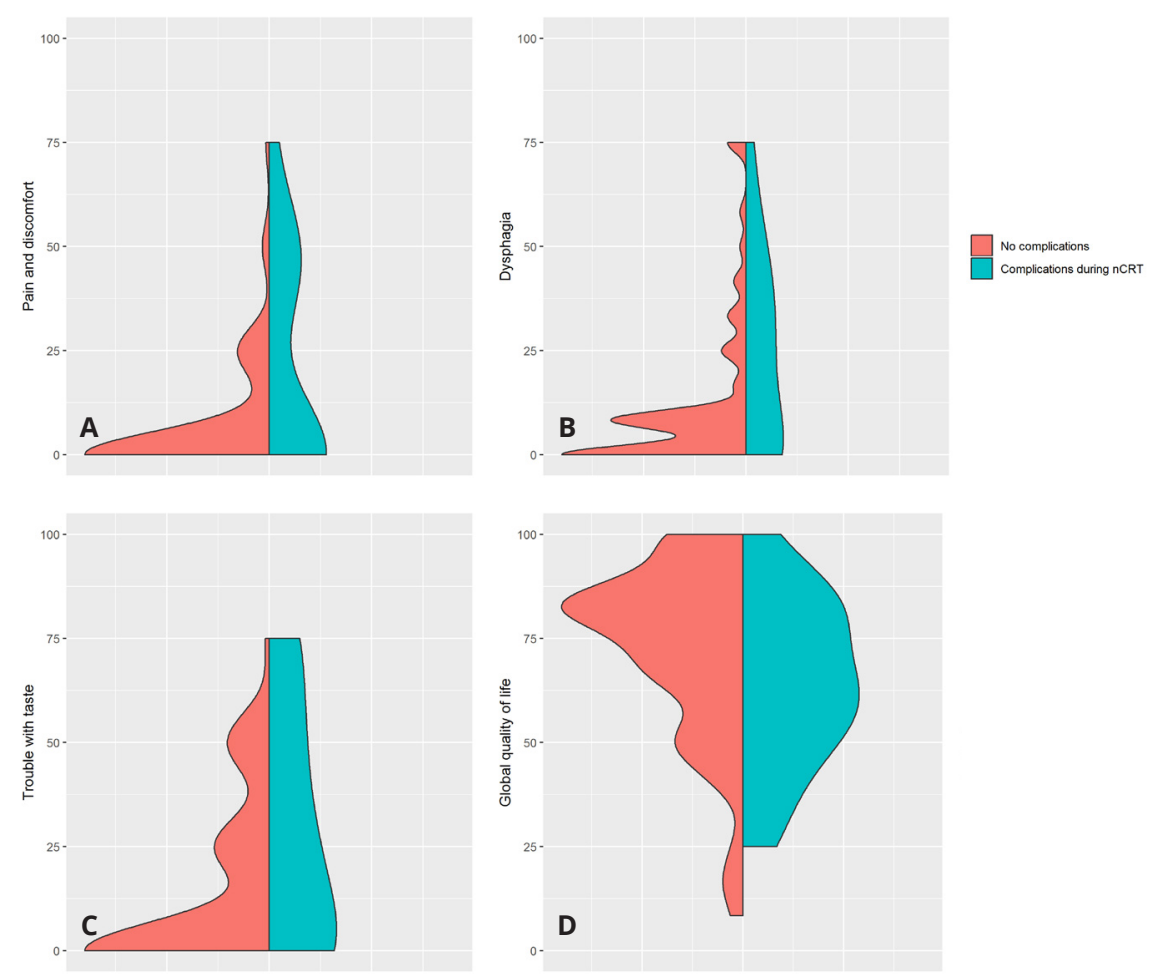

Figure 3. Rotated kernel density plots of pain and discomfort (QLQ-OG25, A), dysphagia (QLQ-OG25, $B)$, trouble with taste (QLQ-OG25, C) and global quality of life (QLQ-C30, D) at follow-up (1 month after neoadjuvant chemoradiotherapy) for patients without adverse events during nCRT ( red) and with adverse events during nCRT (blue).

\section{Discussion}

This study was designed to assess the short-term effect of nCRT and more specifically, adverse events during nCRT, on global and esophageal cancer-specific HRQOL in patients with locally advanced esophageal cancer. In general, the impact of nCRT on global HRQOL 1 month after nCRT was demonstrated to be small. There was a decline in physical and social functioning, and more fatigue and dyspnea. The impact of nCRT on esophageal cancer-specific symptoms was moderately positive on some symptom scales (i.e., less odynophagia and less problems with eating in front of others). Moreover, patients who experienced adverse events during nCRT reported significantly more dysphagia, pain, trouble with taste and declined social functioning. These results extend our knowledge on the impact of nCRT on HRQOL, and are of important clinical value in informed decision-making. The results of our study could be helpful for clinicians to 
meet patients' expectations and facilitate informed decision-making best if HRQOL, cure rates and morbidity are emphasized. ${ }^{22}$

Also, the analysis of HRQOL during the entire treatment pathway of esophageal cancer may identify hidden critical points that may help to plan the best management of these patients. Interestingly, global quality of life was not affected by adverse events during nCRT, but esophageal cancer specific symptoms and social functioning were. These findings suggest that increased attention for fast and adequate treatment of adverse events and symptom management, such as pain management, in the period after nCRT in these patients may be useful to improve HRQOL.

Notably, nearly all patients indicated that they experienced any form of anxiety before and after nCRT. Even though the percentages might be slightly misleading since they indicate the number of patients responding at least with 'a little' in at least one of the items, these findings indicate that it might be beneficial to provide additional psychological support after the first consultation with the treatment team. Emotional well-being did not deteriorate significantly after nCRT or in patients with adverse events, but was lower than in the general Dutch population (mean score at baseline \pm SD: $70 \pm 20$ versus $89 \pm 16$ in the reference population). ${ }^{23,24}$ It is important to realize that baseline HRQOL assessment in the current study was obtained shortly after diagnosis with esophageal cancer. As such, the true effect of the disease and its' treatment on HRQOL can only be adequately assessed when surrogates of true baseline HRQOL scores (in patients without esophageal cancer diagnosis) are obtained from population-based references values. ${ }^{19,23,24}$ This is reflected by the fact that global quality of life was already lower at baseline in the current study population than in the healthy Dutch reference population (mean score at baseline \pm SD: $67 \pm 20$ versus $78 \pm$ 17 in the reference population). ${ }^{23,24}$

It has been demonstrated before that esophageal cancer patients experience a short-term deterioration in HRQOL immediately ( \pm 1 week) after completion of nCRT.7,12 This decline is explained by expected persisting adverse effects and therefore maximum toxicity of chemotherapy and radiotherapy, such as fatigue, esophagitis, and hematologic toxicity. The current study does no longer demonstrate a decline in global HRQOL 1 month after nCRT, but decreased physical functioning and more fatigue persist, even though the effects are smaller than reported immediately after completion of nCRT. ${ }^{7}$ As such, patients might not have reached their full recovery phase from nCRT yet. Decreased physical functioning and increased fatigue are likely also the cause of more dyspnea 1 month after nCRT, as this is expected to be too soon to be a symptom of radiation-induced lung injury. ${ }^{25}$ This finding should also be considered when determining the optimal timing for subsequent surgical resection, with the aim to schedule esophagectomy at least 1 month after nCRT according to a patient's self-evaluation of their physical condition.

Some limitations of the current study deserve attention. HRQOL research by means of 
self-report questionnaires is inherently hampered by selection bias due to non-participation or missing data. Unfortunately, due to logistic reasons, the response rate of the baseline questionnaire in the current study could not be accurately determined. This might have attributed to selection bias as well, although there are no signs that the current study population differs substantially in terms of clinical characteristics from 2 Dutch reference cohorts of patients who underwent neoadjuvant chemoradiotherapy (Supplementary Table 1), nor from the commonly reported Western esophageal cancer population. Furthermore, there is no obvious reason to assume that patients referred directly to our department from outside our center would differ greatly from the patients who are referred from other departments within our center in terms of HRQOL. Also, the response rate of the followup questionnaire was quite high (75.6\%), but associated with the occurrence of adverse events during nCRT. This could imply that the reported declines in the several studied aspects of HRQOL might have been slightly underestimated.

Strengths of the current study include the relatively large and homogenous sample size, as well as the inclusion of both the QLQ-C30 and the QLQ-OG25 questionnaire. It is known that scales in the QLQ-C30 and the QLQ-OG25 are not associated, as these assess different aspects of HRQOL. ${ }^{17}$ Therefore, the use of a modular approach for the assessment of HRQOL in this study is essential to detect both benefits and disadvantages of nCRT.

Another important strength of the study is that the analyses studying the impact of nCRT on HRQOL as well as the analyses studying the impact of adverse events during nCRT on HRQOL account for known confounding variables, resulting in the direct effect of nCRT and adverse events on HRQOL.

In summary, this study is a step towards enhancing the understanding of the short-term impact of nCRT and related adverse events on HRQOL in esophageal cancer patients. The results from this study indicate that the impact of nCRT on general quality of life 1 month after nCRT is small, but effects on physical functioning, as well as fatigue are present 1 month after nCRT. Furthermore, as expected, patients with adverse events during nCRT reported more dysphagia and pain symptoms. These results support informed decisionmaking, especially for patients who fear the negative effect of neoadjuvant treatment. In addition, these results support the incorporation of HRQOL as important endpoints when evaluating treatment efficacy. 


\section{References}

1. Lordick F, Mariette C, Haustermans K, Obermannová R, Arnold D. Oesophageal cancer: ESMO Clinical Practice Guidelines for diagnosis, treatment and follow-up. Ann Oncol. 2016;27(suppl_5):v50-v57.

2. van Hagen P, Hulshof MCCM, van Lanschot JJB, et al. Preoperative Chemoradiotherapy for Esophageal or Junctional Cancer. N Engl J Med. 2012;366(22):2074-2084.

3. Vodicka E, Kim K, Devine EB, Gnanasakthy A, Scoggins JF, Patrick DL. Inclusion of patient-reported outcome measures in registered clinical trials: Evidence from ClinicalTrials.gov (2007-2013). Contemp Clin Trials. 2015;43:1-9.

4. Hoeppner J, Lordick F, Brunner T, et al. ESOPEC: prospective randomized controlled multicenter phase III trial comparing perioperative chemotherapy (FLOT protocol) to neoadjuvant chemoradiation (CROSS protocol) in patients with adenocarcinoma of the esophagus (NCT02509286). BMC Cancer. 2016;16(1):503.

5. Noordman BJ, E Verdam MG, Lagarde SM, et al. Impact of neoadjuvant chemoradiotherapy on healthrelated quality of life in long-term survivors of esophageal or junctional cancer: results from the randomized CROSS trial. Ann Oncol. 2018;29:445-451.

6. Noordman BJ, Verdam MGE, Lagarde SM, et al. Effect of Neoadjuvant Chemoradiotherapy on HealthRelated Quality of Life in Esophageal or Junctional Cancer: Results From the Randomized CROSS Trial. J Clin Oncol. 2018;36(3):268-275.

7. Blazeby JM, Sanford E, Falk SJ, Alderson D, Donovan JL. Health-related quality of life during neoadjuvant treatment and surgery for localized esophageal carcinoma. Cancer. 2005;103(9):1791-1799.

8. Derogar M, Orsini N, Sadr-Azodi O, Lagergren P. Influence of major postoperative complications on health-related quality of life among long-term survivors of esophageal cancer surgery. J Clin Oncol. 2012;30(14):1615-1619.

9. Rutegård M, Lagergren J, Rouvelas I, Lindblad M, Blazeby JM, Lagergren P. Population-based study of surgical factors in relation to health-related quality of life after oesophageal cancer resection. Br J Surg. 2008;95(5):592-601.

10. Scarpa M, Saadeh LM, Fasolo A, et al. Health-Related Quality of Life in Patients with Oesophageal Cancer: Analysis at Different Steps of the Treatment Pathway. J Gastrointest Surg. 2013;17(3):421-433.

11. Kauppila JH, Johar A, Lagergren P. Postoperative Complications and Health-related Quality of Life 10 Years After Esophageal Cancer Surgery. Ann Surg. 2020;271(2):311-316.

12. Haj Mohammad N, De Rooij S, Hulshof M, et al. Activities of daily living and quality of life during treatment with neoadjuvant chemoradiotherapy and after surgery in patients with esophageal cancer. J Surg Oncol. 2016;114(6):684-690.

13. National guideline esophageal cancer (version 3.1). 2015. http://www.oncoline.nl/oesofaguscarcinoom. Accessed March 21, 2018.

14. Aaronson NK, Ahmedzai S, Bergman B, et al. The European Organization for Research and Treatment of Cancer QLQ-C30: A Quality-of-Life Instrument for Use in International Clinical Trials in Oncology. J Natl Cancer Inst. 1993;85(5):365-376.

15. Blazeby JM, Williams MH, Brookes ST, Alderson D, Farndon JR. Quality of life measurement in patients with oesophageal cancer. Gut. 1995;37(4):505-508.

16. Giesinger JM, Kieffer JM, Fayers PM, et al. Replication and validation of higher order models demonstrated that a summary score for the EORTC QLQ-C30 is robust. J Clin Epidemiol. 2016;69:79-88.

17. Lagergren $\mathrm{P}$, Fayers $\mathrm{P}$, Conroy T, et al. Clinical and psychometric validation of a questionnaire module, the EORTC QLQ-OG25, to assess health-related quality of life in patients with cancer of the oesophagus, the oesophago-gastric junction and the stomach. Eur J Cancer. 2007;43(14):2066-2073.

18. Fayers P, Aaronson N, Bjordal K, Al. E. EORTC QLQ-C30 Scoring Manual, 3rd Edition. Brussels: EORTC; 2011.

19. van der Schaaf M, Derogar M, Lagergren P. Reference values of oesophago-gastric symptoms (EORTC QLQ-OG25) in a population-based setting. Eur J Cancer. 2012;48(11):1602-1607.

20. Cocks K, King MT, Velikova G, et al. Evidence-based guidelines for interpreting change scores for the European Organisation for the Research and Treatment of Cancer Quality of Life Questionnaire Core 30. Eur J Cancer. 2012;48(11):1713-1721. 
21. Osoba D, Rodrigues G, Myles J, Zee B, Pater J. Interpreting the significance of changes in health-related quality-of-life scores. J Clin Oncol. 1998;16(1):139-144.

22. Thrumurthy SG, Morris JJA, Mughal MM, Ward JB. Discrete-choice preference comparison between patients and doctors for the surgical management of oesophagogastric cancer. Br J Surg. 2011;98(8):11241131.

23. van de Poll-Franse L V, Mols F, Gundy CM, et al. Normative data for the EORTC QLQ-C30 and EORTCsexuality items in the general Dutch population. Eur J Cancer. 2011;47(5):667-675.

24. Mols F, Husson O, Oudejans M, Vlooswijk C, Horevoorts N, van de Poll-Franse L V. Reference data of the EORTC QLQ-C30 questionnaire: five consecutive annual assessments of approximately 2000 representative Dutch men and women. Acta Oncol (Madr). 2018;57(10):1381-1391.

25. Hanania AN, Mainwaring W, Ghebre YT, Hanania NA, Ludwig M. Radiation-Induced Lung Injury: Assessment and Management. Chest. 2019;156(1):150-162. 


\section{SUPPLEMENTARY MATERIAL}

Supplementary Table 1. Clinical characteristics of the study population (patients who started neoadjuvant chemoradiotherapy for esophageal cancer and returned the baseline questionnaire) compared to the clinical characteristics of 2 Dutch reference cohorts.

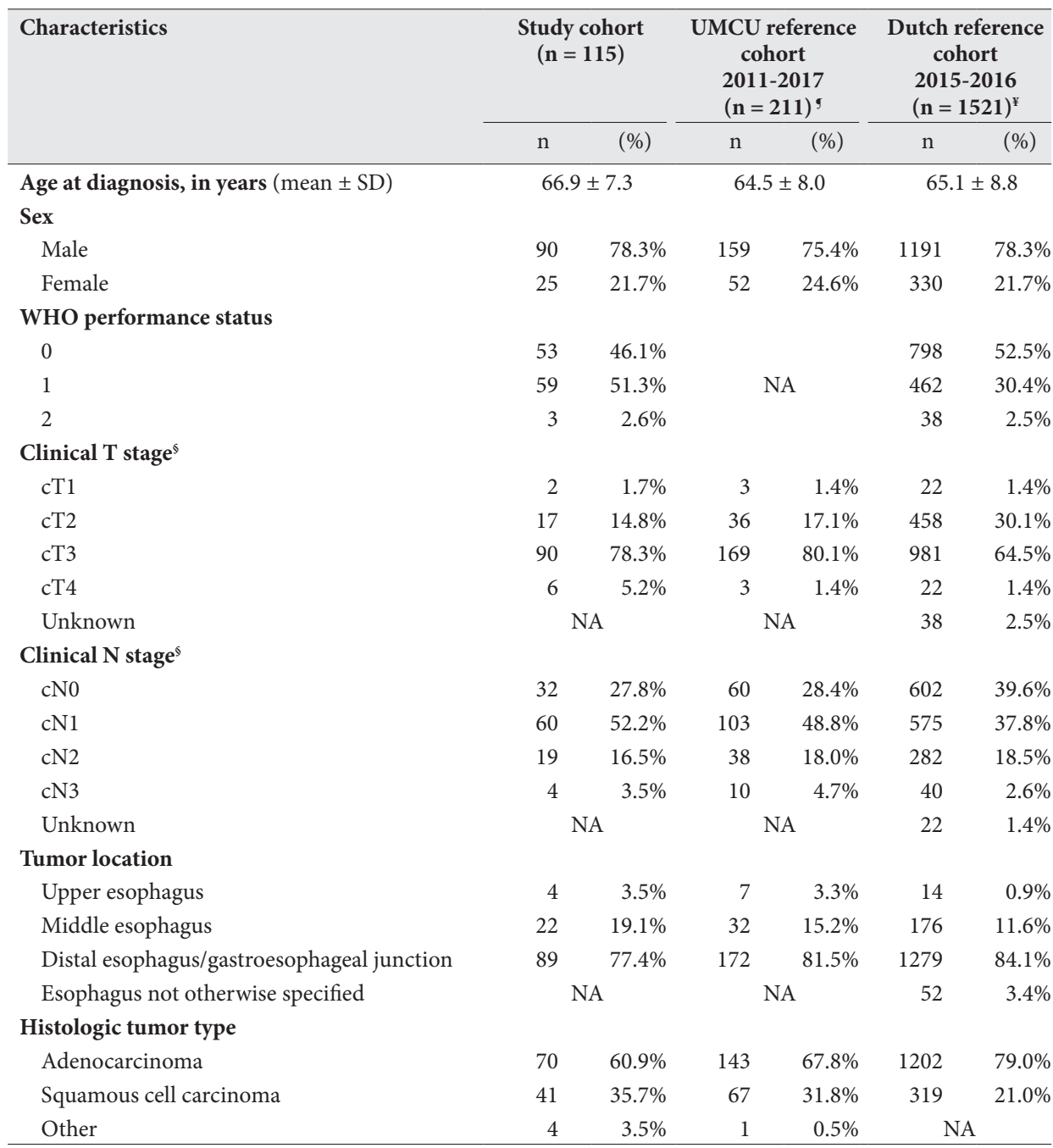

NA not applicable/available; SD standard deviation; WHO World Health Organization

$\checkmark$ Clinical T and N stage are based on AJCC TNM $7^{\text {th }}$ edition.

S Reference cohort of patients who underwent neoadjuvant chemoradiotherapy followed by esophagectomy for esophageal cancer at the University Medical Center Utrecht during 2011-2017.

¥ Reference cohort of patients who started neoadjuvant chemoradiotherapy for esophageal cancer in the Netherlands in 2015-2016, based on the Netherlands Cancer Registry (as reported on by Borggreve et al. Frequency of surgical resection after starting neoadjuvant chemoradiotherapy in patients with esophageal cancer: A population-based cohort study. European Journal of Surgical Oncology 2019). 
Supplementary Table 2. Mean scores and available number of patient-reported outcomes per health-related quality of life (HRQOL)-scale before starting neoadjuvant chemoradiotherapy (baseline) and 1 month after neoadjuvant chemoradiotherapy (follow-up).

\begin{tabular}{|c|c|c|c|c|c|c|}
\hline \multirow[t]{2}{*}{ Quality of life dimension } & \multicolumn{3}{|c|}{ Baseline ( $n=115)$} & \multicolumn{3}{|c|}{ Follow-up $(n=85)$} \\
\hline & n & $\begin{array}{l}\text { Absolute } \\
\text { mean score }\end{array}$ & SD & $\mathbf{n}$ & $\begin{array}{l}\text { Absolute } \\
\text { mean score }\end{array}$ & SD \\
\hline \multicolumn{7}{|l|}{ QLQ-C30 } \\
\hline Global quality of life $\mathrm{e}^{\S}$ & 115 & 67 & 20 & 85 & 71 & 21 \\
\hline \multicolumn{7}{|l|}{ Functional scales $s^{\S}$} \\
\hline Physical functioning & 115 & 85 & 17 & 85 & 78 & 20 \\
\hline Role functioning & 115 & 77 & 27 & 85 & 73 & 29 \\
\hline Emotional functioning & 114 & 70 & 20 & 85 & 76 & 23 \\
\hline Cognitive functioning & 114 & 84 & 20 & 85 & 85 & 18 \\
\hline Social functioning & 114 & 83 & 23 & 85 & 77 & 28 \\
\hline \multicolumn{7}{|l|}{ Symptom scales } \\
\hline Fatigue & 114 & 30 & 24 & 85 & 34 & 24 \\
\hline Nausea and vomiting & 114 & 11 & 17 & 85 & 9 & 21 \\
\hline Pain & 115 & 22 & 22 & 85 & 19 & 27 \\
\hline \multicolumn{7}{|l|}{ Single-item symptom scores } \\
\hline Dyspnea & 115 & 13 & 20 & 84 & 21 & 25 \\
\hline Insomnia & 114 & 26 & 28 & 85 & 23 & 30 \\
\hline Loss of appetite & 114 & 21 & 30 & 83 & 18 & 29 \\
\hline Constipation & 113 & 15 & 26 & 84 & 11 & 21 \\
\hline Diarrhea & 114 & 5 & 15 & 85 & 9 & 21 \\
\hline Financial difficulties & 112 & 9 & 25 & 84 & 9 & 24 \\
\hline QLQ-C30 Summary Score ${ }^{\S \Psi}$ & 113 & 81 & 12 & 82 & 81 & 16 \\
\hline \multicolumn{7}{|l|}{ QLQ-OG25 } \\
\hline \multicolumn{7}{|l|}{ Multi-item symptom scales } \\
\hline Dysphagia & 115 & 24 & 20 & 84 & 14 & 20 \\
\hline Eating restrictions & 115 & 32 & 23 & 83 & 22 & 23 \\
\hline Reflux & 115 & 5 & 13 & 83 & 4 & 11 \\
\hline Odynophagia & 113 & 29 & 23 & 80 & 13 & 18 \\
\hline Pain and discomfort & 114 & 14 & 18 & 84 & 9 & 18 \\
\hline Anxiety & 115 & 46 & 19 & 85 & 37 & 19 \\
\hline \multicolumn{7}{|l|}{ Single-item symptom scores } \\
\hline Eating in front of others & 112 & 21 & 26 & 81 & 8 & 18 \\
\hline Dry mouth & 115 & 13 & 21 & 85 & 16 & 22 \\
\hline Trouble with taste & 114 & 8 & 17 & 85 & 16 & 23 \\
\hline Body image & 114 & 8 & 16 & 85 & 11 & 21 \\
\hline Trouble swallowing saliva & 115 & 12 & 23 & 85 & 10 & 20 \\
\hline Choked when swallowing & 115 & 6 & 14 & 85 & 6 & 14 \\
\hline Trouble with coughing & 114 & 20 & 20 & 83 & 22 & 22 \\
\hline Trouble talking & 115 & 4 & 11 & 83 & 5 & 14 \\
\hline Weight loss & 115 & 23 & 27 & 84 & 18 & 22 \\
\hline
\end{tabular}

SD standard deviation

$\$$ Score range 0-100. Higher scores represent a better quality of life or higher level of functioning.

- Score range 0-100. Higher scores represent more severe symptoms or problems.

₹ Mean of all scores except for global quality of life and financial difficulties. 


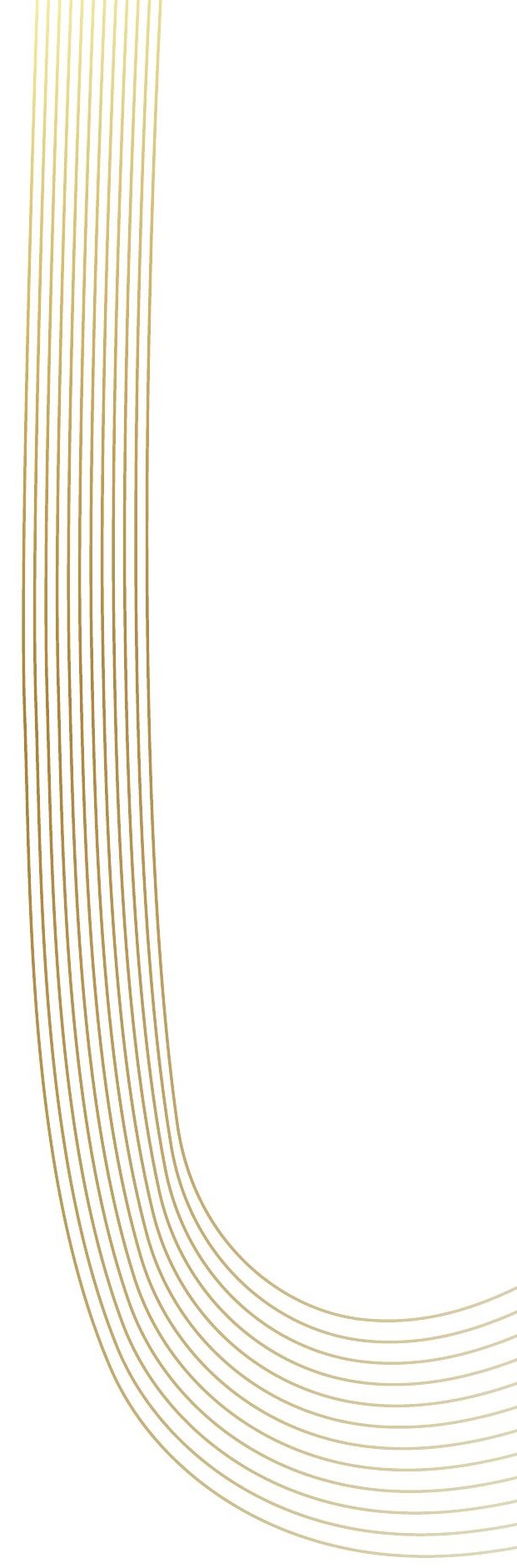


Chapter 3

Quantification of variations in intra-fraction motion
of esophageal tumors over the course of neoadjuvant
chemoradiotherapy based on cine-MRI

Physics in Medicine \& Biology. 2018;63(14):145019.

S.E. Heethuis, A.S. Borggreve, L. Goense, P.S.N. van Rossum, S. Mook, R. van Hillegersberg, J.P. Ruurda, G.J. Meijer, J.J.W. Lagendijk, A.L.H.M.W. van Lier 


\section{Abstract}

\section{Purpose}

To noninvasively quantify variations in intra-fraction motion of esophageal tumors over the course of neoadjuvant chemoradiotherapy (nCRT) using 2D cine-magnetic resonance imaging (MRI) series.

\section{Material and Methods}

Patients treated with nCRT for esophageal cancer underwent 6 MRI scans. Scans were acquired prior to the start of nCRT, followed by weekly MRI scans during nCRT. Cine-MRI series were acquired in the coronal and sagittal plane $(\approx 1.6 \mathrm{~Hz})$. To be able to quantify intra-fraction motion over a longer time period, a second cine-MRI series was performed after 10 minutes. Tumor motion was assessed in cranio-caudal (CC), anterior-posterior (AP) and left-right (LR) direction. Motion patterns were analyzed for the presence of deep inhales and tumor drift.

\section{Results}

A total of 232 cine-MRI series of 20 patients were analyzed. The largest tumor motion was found in CC direction, with a mean peak-to-peak motion of $12.7 \mathrm{~mm}$ (standard deviation [SD] 5.6), followed by a mean peak-to peak motion in AP direction of $3.8 \mathrm{~mm}$ (SD 2.0) and in LR direction of $2.7 \mathrm{~mm}$ (SD 1.3). The CC intra-fraction tumor motion can differ extensively between and within patients. Deep inhales were present in 6 of 232 scans (3\%). After exclusion of these scans, mean CC peak-to-peak motion was $12.3 \mathrm{~mm}$ (SD 5.2). Correction for tumor drift showed a further reduction to $11.0 \mathrm{~mm}$ (SD 4.6). Despite correction for tumor drift, large variation in tumor motion occurred within patients during treatment. Mean tumor drift during the 10 minute interval between the 2 series was 1.5 $\mathrm{mm}$ (SD 1.8), with a maximum of $11.6 \mathrm{~mm}$.

\section{Conclusions}

Intra-fraction tumor motion was found to be highly variable between and within patients with esophageal cancer over the course of nCRT. Correction for deep inhales and tumor drift reduced peak-to-peak motion. The stochastic nature of both deep inhales and tumor drift indicates that real-time tumor motion management during radiotherapy is a prerequisite to safely reduce treatment margins. 


\section{Introduction}

Neoadjuvant chemoradiotherapy (nCRT) followed by surgical resection is widely accepted as standard treatment with curative intent for patients with resectable esophageal cancer. ${ }^{1-3}$ Due to the presence of positioning uncertainties and respiratory motion, substantial CTVto-PTV margins (typically ranging from $10 \mathrm{~mm}$ to $15 \mathrm{~mm}$ ) are currently applied. ${ }^{1}$ Smaller CTV-to-PTV margins would result in a reduced radiation dose to the surrounding healthy tissues, and would thereby reduce short- and long-term complications. Furthermore, smaller margins could be used to increase the dose to the tumor, while maintaining the dose constraints for healthy tissues that are currently used. In order to develop highly conformal treatment plans with adequate tumor coverage, the irradiation field needs to be adapted to tumor movement. As such, information about (variations in) tumor movement over the course of nCRT is desired.

To date, several imaging modalities have been used to assess tumor motion in esophageal cancer patients. Drawbacks of these studies using four dimensional computed tomography (4DCT) to quantify esophageal tumor motion $^{4-7}$ are the additional exposure to radiation and the need for invasive placement of fiducial markers. Furthermore, 4DCT studies only provide information on the average tumor motion as observed during CT acquisition, but not on variations in the tumor motion during scanning. This is due to the reconstruction of 4 DCT scans in which the tumor motion is binned in a defined number of respiratory phases or amplitudes after acquisition, resulting in the reconstruction of only one breathing cycle in $4 \mathrm{DCT}$. Therefore it is not possible to measure tumor drift with $4 \mathrm{DCT}$, as measurement of this process requires reconstruction of multiple breathing cycles.

Esophageal tumor motion can also be visualized with magnetic resonance imaging (MRI). In cine-MRI, subsequent $2 \mathrm{D}$ images are acquired with a high temporal resolution, which is widely used to study motion patterns in different tumor sites. ${ }^{8-10}$ A mean cranio-caudal peak-to-peak motion of $13.3 \mathrm{~mm}$ with a wide range of 2.7 to $24.5 \mathrm{~mm}$ was found using cine-MRI, in which the mobility of distal tumors was generally larger than that of more proximal tumors. ${ }^{11}$

In previous studies either four-dimensional cone-beam CT (4D-CBCT) was used over the course of treatment to quantify variations in tumor motion, or MRI was used at a single time point prior to treatment. As mentioned above, the disadvantage of 4DCT imaging is that it does not give information about possible tumor drift. Therefore, the purpose of our observational study was to quantify variations in intra-fraction esophageal tumor motion over the course of nCRT within the same patient, using weekly cine-MRI series. Insight in motion patterns of esophageal tumors could be used to determine which motion management strategy (e.g. tumor tracking, tumor gating, dynamic planning) $)^{12,13}$ may be the most appropriate for on-line MRI-guided radiotherapy. ${ }^{14,15}$ Furthermore, if tumor 
motion prior to treatment is a reliable indicator for tumor motion during treatment, personalized PTV margins could be used in future clinical practice.

\section{Methods and Materials}

\section{Study population}

Patients with histologically confirmed esophageal cancer who were planned to receive nCRT followed by surgery were eligible for inclusion. Neoadjuvant chemoradiotherapy consisted of 5 weeks of treatment, involving weekly intravenous administration of carboplatin and paclitaxel with concurrent radiotherapy ( $41.4 \mathrm{~Gy}$ in 23 fractions of $1.8 \mathrm{~Gy}$ ) according to the CROSS regimen. ${ }^{1}$ Standard diagnostic work-up consisted of endoscopy with biopsies of the primary tumor site, as well as integrated ${ }^{18} \mathrm{~F}-\mathrm{FDG}$-PET/CT for clinical staging. Patients with contraindications for MRI were not eligible for inclusion. The study was approved by the local medical ethics committee and written informed consent was obtained from all patients.

\section{MRI acquisition}

MRI images were acquired at 6 time points; prior to the start of nCRT, followed by weekly MR scans during the course of nCRT. All images were acquired on a 1.5T Philips Ingenia (Best, the Netherlands), using the Anterior/Posterior (28 channel) receive coils. Patients were positioned in supine position with both arms next to the body. Respiratory-triggered sagittal and transverse anatomical $\mathrm{T}_{2}$-weighted scans were acquired with a multi-slice turbo spin echo sequence $\left(\mathrm{tT}_{2} \mathrm{~W}: \mathrm{TR} / \mathrm{TE}=1983 / 100 \mathrm{~ms}, \mathrm{sT} \mathrm{W}_{2} \mathrm{~W}: \mathrm{TR} / \mathrm{TE}=1432 / 100 \mathrm{~ms}\right.$, resolution $=0.67 \times 0.67 \times 4 \mathrm{~mm}^{3}$ ). These scans served as reference to plan 2 cine-MRI series: one coronal and one sagittal cine-MRI series To prevent substantial out-of-plane motion the scanning plane of the coronal cine-MRI series was angulated, such that it was aligned with the largest extent of the tumor in the sagittal $\mathrm{T}_{2}$-weighted scan. For the sagittal cine-MRI series, the scanning plane was positioned through the center of the tumor, which was identified using the transverse $\mathrm{T}_{2}$-weighted scan. The images were both acquired with a resolution of $2.01 \times 2.01 \mathrm{~mm}$ and $5 \mathrm{~mm}$ slice thickness, during 46 seconds of free breathing $(1.6 \mathrm{~Hz})$ for the coronal and 43 seconds $(1.7 \mathrm{~Hz})$ for the sagittal series. In order to quantify the difference in intra-fraction tumor motion and drift within one scanning session, both coronal and sagittal cine-MRI series were acquired twice during each scanning session: the first series was initiated after approximately 20 minutes of scanning and the second series after scanning a diffusion-weighted (DW-) MR series (10 minutes). These DW-MR scans included a b-value of $800 \mathrm{~s} / \mathrm{mm}^{2}$ (SPIR fat suppression, resolution $=3.25 \times 3.25 \times 4 \mathrm{~mm}^{3}$, bandwidth per pixel $=22.9 \mathrm{~Hz}){ }^{16}$ 


\section{Motion characterization}

The primary tumor was delineated on the initial b800 $\mathrm{s} / \mathrm{mm}^{2} \mathrm{DW}-\mathrm{MRI}$, using an automated contouring algorithm based on clustering (ITK-SNAP ${ }^{17}$ ). Afterwards, this delineation was verified using the transverse $\mathrm{T}_{2} \mathrm{~W}$ scan $\left(\mathrm{tT}_{2} \mathrm{~W}\right)$. For each of the subsequent time points (2 to 6) a rigid registration between the $\mathrm{tT}_{2} \mathrm{~W}$ scan and the $\mathrm{tT}_{2} \mathrm{~W}$ baseline scan was performed in Elastix (Version 4.7). ${ }^{18}$ Using this registration, the tumor delineation of the first time point was propagated to the other time points ( 2 to 6 ) and manually adjusted if necessary (e.g. in case of shrinkage of the esophageal circumference). Next, all tumor delineations were propagated to the corresponding 2D cine-MRI series. An optical flow algorithm (RealTITracker ${ }^{19,20}$ ) was used to quantify tumor motion on the cine-MRI series. Corresponding motion vector fields were calculated for each frame with respect to a reference frame and evaluated within the delineated tumor volume. The reference frame was manually selected from 6 consecutive frames in such a way that the shape of the tumor resembled the tumor delineation as performed on the initial b800 scan as closely as possible. Motion was evaluated in cranio-caudal (CC), left-right (LR) and anterior-posterior (AP) directions (Matlab, The Mathworks Inc, Natick, Massachusetts, USA). The angulation of the coronal scanning plane was taken into account for the calculation of the CC-motion, such that the true CC-motion was calculated. Peak-to-peak amplitudes of tumor motion were calculated in all three directions. Furthermore, the smallest range to include $95 \%$ of the data points (excluding 5\% most extreme data points) was calculated (C95). This C95 is a more robust measure for tumor motion as it excludes outliers caused by sporadic movement (e.g. sneezing, hiccups) as seen on the cine-MRI series. CC-motion patterns analyses were divided into short- and long-term intra-fraction motion (see Figure 1 for schematic overview of timeline and terminology).

Short-term intra-fraction motion was defined as the motion occurring within one cine-MRI series of 45 seconds, in which the presence of deep inhales and tumor drifting or irregular motion patterns were quantified. To estimate the effect of deep inhales on the motion curves, the minimum value $\left(0^{\text {th }}\right.$ percentile, $\left.\mathrm{P} 0\right)$ and $10^{\text {th }}$ percentile $(\mathrm{P} 10)$ were compared. No large $\mathrm{P} 10-\mathrm{P} 0$ differences were expected in a regular breathing curve. However, in a curve in which a deep inhale occurs (presenting as a negative peak in a motion curve), a large difference in P10-P0 was expected.

To determine the presence of tumor drift in motion curves, drift lines were estimated. These drift lines were defined as the mid-position (mean) between consecutively found local maxima and minima in the motion curve. This drift line was subtracted from the motion curve, after which the difference in peak-to-peak and C95 amplitude for both curves functioned as a measure for the presence of a drift.

Long-term intra-fraction motion was defined as the motion which occurred within the 10 minute time-interval between the 2 cine-MRI series. This was quantified as the shift between 
the initial drift lines (first 6 data-points) of both the first and second cine-MRI series. This can be interpreted as a conservative surrogate for the displacement of the esophageal tumor between set-up of the patient and final dose delivery. In which a positive long-term drift represents a movement in cranial direction.

\section{Statistical analysis}

This study was of descriptive nature and therefore no formal sample size calculation was performed. All visual representations were generated using GraphPad Prism 6.07 software (GraphPad Software, La Jolla, California, USA) and statistical analysis was performed in SPSS Statistics version 22 (IBM Corp., Armonk, New York, USA). The mean and standard deviation (SD) of the peak-to-peak and C95 amplitude for the whole group were calculated.

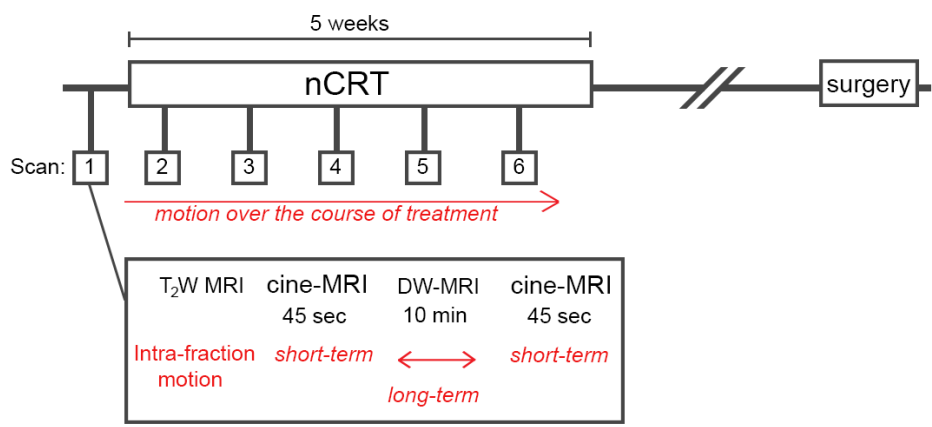

Figure 1. Overview of treatment and scanning timeline including a schematic representation of terminology used for indication of intra-fraction motions quantified in this study.

\section{Results}

\section{Study population}

A total of 25 consecutive patients were included, with MR scans acquired between April 2016 and October 2017. Five patients were excluded for analysis due to small tumor volume $(<7 \mathrm{ml}, \mathrm{n}=1)$, poor tumor visibility on MRI $(\mathrm{n}=3)$ or withdrawal of informed consent $(\mathrm{n}=1)$, resulting in a total of 20 patients suitable for analysis. In 3 of the included 20 patients, one of the weekly MR-scans during nCRT was incomplete or missing due to the condition of the patient. In 2 patients the second cine-MR series was not available at one time point. This led to a total amount of 232 acquired cine-MRI series for the total study population. All patients were male (20/20) with a mean age of 66.6 years (SD 6.9). Two patients had a mid-esophageal tumor (patient 1 and 2), 15 patients a distal esophageal tumor (patients 
3-17), whereas the remaining 3 patients had a gastroesophageal junction tumor (patients 18-20). Patient and tumor characteristics are summarized in Table 1.

\section{Tumor motion}

A coronal cine-MRI frame together with the estimated CC and LR motion is shown in Figure 2. This figure shows excellent visualization of the thorax and abdomen at 1.5T, enabling motion characterization. The largest intra-fraction esophageal tumor motion was found in CC direction with a mean peak-to-peak motion of $12.7 \mathrm{~mm}$ (SD 5.6, range 4.433.7). The mean AP peak-to-peak motion was $3.8 \mathrm{~mm}$ (SD 2.0, range 0.7-10.2). The least motion was seen in LR direction, with a mean peak-to-peak of $2.7 \mathrm{~mm}$ (SD 1.3, range 0.7-8.3). The calculated peak-to-peak and C95 in the CC-direction for the whole group is summarized in Table 2. Since CC-motion was $22 \%$ less on average when calculated on sagittal images, analyses were only performed on the coronal cine-MRI series.

Table 1. Patient characteristics

\begin{tabular}{|c|c|c|}
\hline \multirow[b]{2}{*}{ Characteristic } & \multicolumn{2}{|c|}{ Full cohort $(n=20)$} \\
\hline & $\mathrm{n}$ & $\%$ \\
\hline \multicolumn{3}{|l|}{ Gender } \\
\hline Male & 20 & $(100 \%)$ \\
\hline Age (at start treatment), years (mean $\pm S D$ ) & \multicolumn{2}{|c|}{$66.6 \pm 6.9$} \\
\hline \multicolumn{3}{|l|}{ Clinical T-status } \\
\hline $\mathrm{T} 2$ & 1 & $(5 \%)$ \\
\hline $\mathrm{T} 3$ & 19 & $(95 \%)$ \\
\hline \multicolumn{3}{|l|}{ Clinical N-status } \\
\hline No & 7 & $(35 \%)$ \\
\hline N1 & 9 & $(45 \%)$ \\
\hline $\mathrm{N} 2$ & 3 & $(15 \%)$ \\
\hline N3 & 1 & $(5 \%)$ \\
\hline \multicolumn{3}{|l|}{ Histopathology } \\
\hline Squamous cell carcinoma & 4 & $(20 \%)$ \\
\hline Adenocarcinoma & 15 & $(75 \%)$ \\
\hline Undifferentiated carcinoma & 1 & $(5 \%)$ \\
\hline \multicolumn{3}{|l|}{ Location } \\
\hline Proximal third of esophagus & 0 & $(0 \%)$ \\
\hline Middle third of esophagus & 2 & $(10 \%)$ \\
\hline Distal third of esophagus & 15 & $(75 \%)$ \\
\hline Gastroesophageal junction & 3 & $(15 \%)$ \\
\hline \multicolumn{3}{|l|}{ Volume planning GTV $(\mathrm{ml})$} \\
\hline Mean $( \pm \mathrm{SD})$ & \multicolumn{2}{|c|}{$50.9 \pm 25.5$} \\
\hline Range & \multicolumn{2}{|c|}{$19.0-126.3$} \\
\hline
\end{tabular}

GTV gross tumor volume, $S D$ standard deviation 

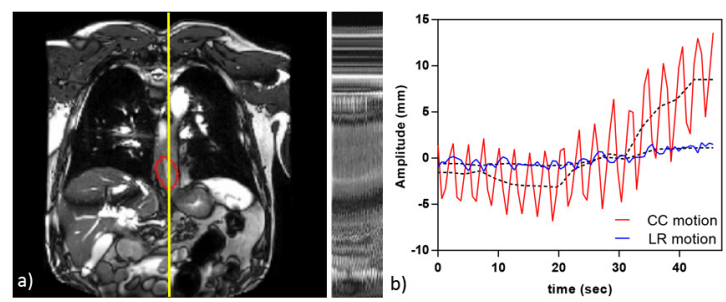

Figure 2. (A) A cine-MRI scan of a patient with the tumor delineation in red. On the right side the 75 time frames are visible corresponding to the location of the yellow line in the image. (B) Amplitude of the tumor motion in cranio-caudal (CC, red) and left-right direction (LR, blue). The black dashed lines show the estimated drift lines.

Table 2. Cranio-caudal tumor motion

\begin{tabular}{|c|c|c|c|c|c|c|c|c|c|}
\hline \multirow[b]{2}{*}{ Motion } & \multicolumn{3}{|c|}{$\begin{array}{l}\text { All data } \\
(n=232)\end{array}$} & \multicolumn{3}{|c|}{$\begin{array}{c}\text { Deep inhale exclusion } \\
(n=226)\end{array}$} & \multicolumn{3}{|c|}{$\begin{array}{l}\text { Drift corrected } \\
\quad(n=226)\end{array}$} \\
\hline & Mean & $\mathrm{SD}$ & Range & Mean & SD & Range & Mean & SD & Range \\
\hline Peak-to-peak (mm) & 12.7 & 5.6 & $4.4-33.7$ & 12.3 & 5.2 & $4.4-28.1$ & 11.0 & 4.6 & $4.1-26.6$ \\
\hline C95 (mm) & 10.9 & 4.8 & $3.5-27.3$ & 10.6 & 4.6 & $3.5-26.7$ & 9.8 & 4.0 & $3.9-25.4$ \\
\hline
\end{tabular}

C95 the smallest range to include $95 \%$ of the data points (excluding $5 \%$ most extreme data points), SD standard deviation

Typical CC tumor motion curves are presented in Figure 3. Curves with long-term intrafraction drift (patient 1) and short-term intra-fraction drift (patient 4), as well as regular (patient 2) and irregular motion behavior (patient 15) are depicted. The variety of tumor motion for the entire patient group is visualized in Figure $4 \mathrm{a}$, in which the peak-to-peak amplitude is shown in each patient for the 2 scans for each time point separately. As illustrated by Figure $4 \mathrm{a}$, the average intra-fraction tumor motion can differ extensively between patients, and large variations of intra-fraction motion are seen within some patients. Furthermore, it indicates that amplitude prediction for tumor motion throughout the treatment course based on the initial two baseline series (blue line represent mean initial amplitude), creates a risk of severe overestimation (patient 7) or underestimation (patient 4).

\section{Short-term intra-fraction motion}

Analysis of the CC motion patterns based on the P10 and P0 revealed presence of deep inhales in 6 of the 232 series (3\%). In these 6 cine-MRI series, the magnitude of the tumor motion during these inhales was 2 times larger than that of the remaining tumor motion, and the P10-P0 value was more than 3 times the standard deviation above the mean of all 232 series. Results of the short-term drift correction, using the drift lines within the series, 

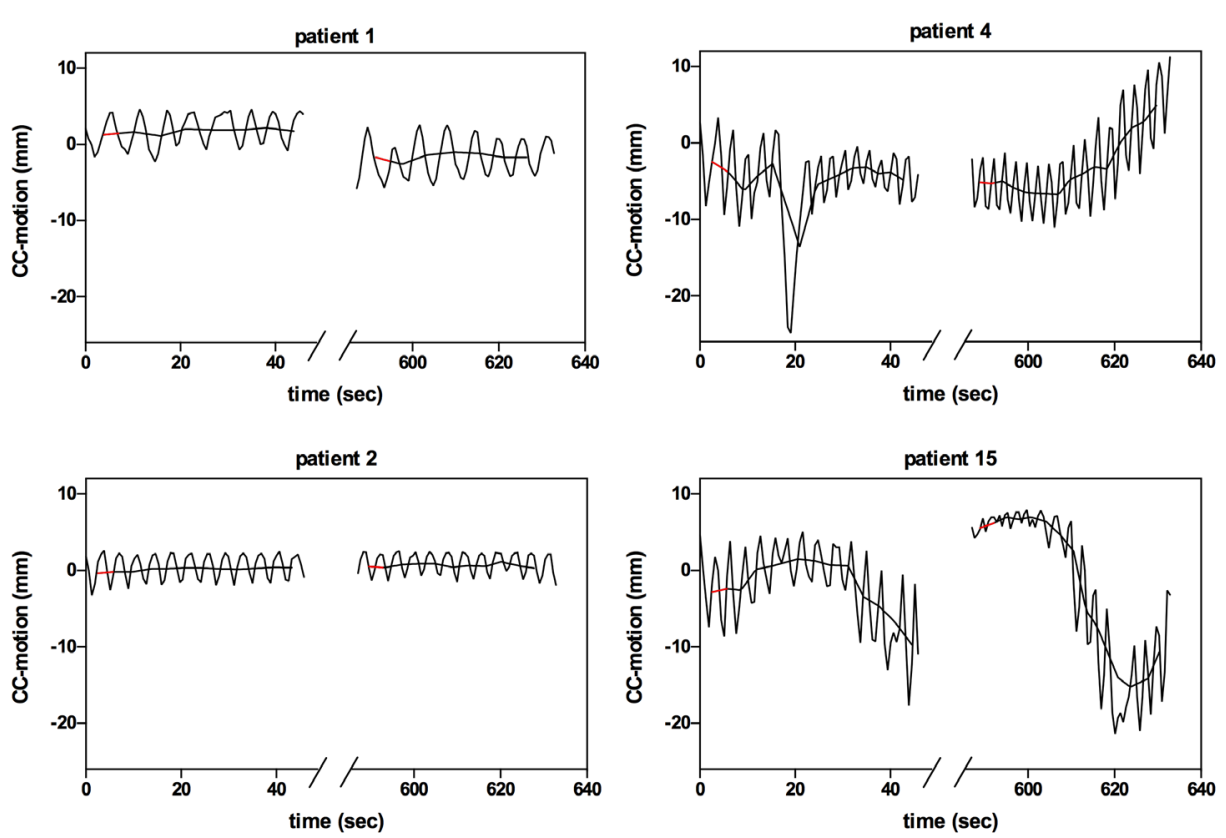

Figure 3. Four examples of motion curves with different behavior are visualized. Patient 1: a long-term drift between series. Patient 2: very regular motion. Patient 4: a deep inhale and short-term drift. Patient 15: irregular motion behavior in both series. The first 6 data-points in each drift line, indicated in red, were used to quantify the long-term drift.

are visualized in Figure 4b. A mean reduction in peak-to-peak amplitude of $1.3 \mathrm{~mm}$ (SD 1.8 ) was found. The largest short-term drift was present in patient $15(-12.4 \mathrm{~mm})$, which was verified by visual inspection of the cine-MRI series and is also depicted in Figure 3 (second series). Since a drift line correction does not compensate for deep inhales, the 3 large amplitudes in the CC motion of patient 7 - caused by 3 deep inhales - remained. As the presence of these deep inhales obscured the effect of the drift line correction, cine-MRI series including deep inhales were excluded. Peak-to-peak and C95 motion amplitudes, as well as the corresponding drift-corrected amplitudes after exclusion of series with deep inhales are presented in Table 2. A mean peak-to-peak motion of $11.0 \mathrm{~mm}$ (SD 4.6, range 4.1-26.6) was found after exclusion of deep inhales and drift correction. The decrease in peak-to-peak motion after deep inhale exclusion and drift correction is also visualized in Figure 5. After drift correction, $49 \%$ of the scans showed a maximum peak-to-peak amplitude smaller than $10 \mathrm{~mm}$, compared to $38 \%$ before drift correction. Before any correction the peak-to-peak amplitude of $95 \%$ of the fractions fell within $24.6 \mathrm{~mm}$, this measure of intra-fraction motion was reduced with $5.2 \mathrm{~mm}$ if deep inhale exclusion and drift correction was applied. 

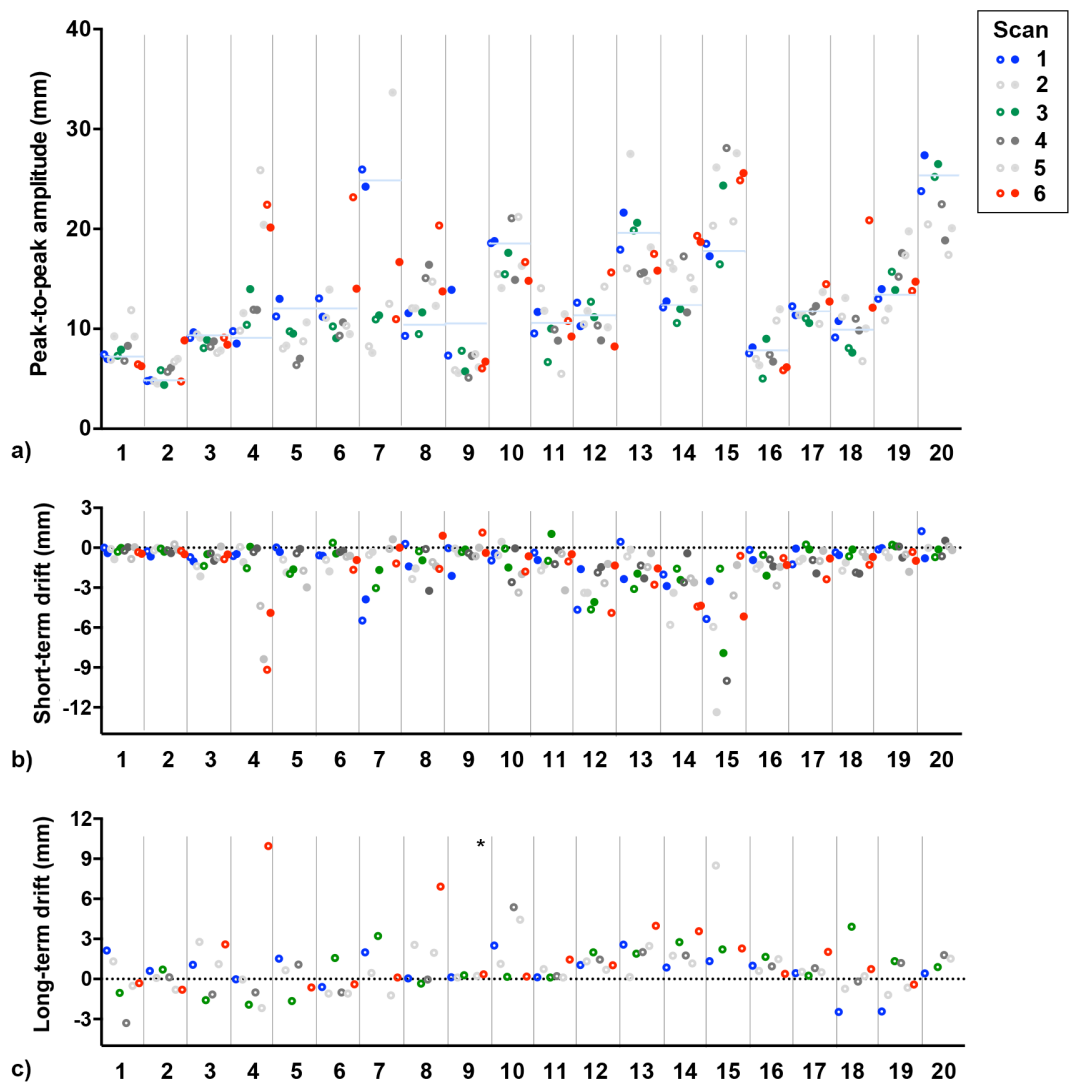

Figure 4. Intra-fraction motion for the six different time points visualized for each patient. For every time point the peak-to-peak cranio-caudal-motion is shown for both scans within the session. The second series is indicated with a closed circle. All patients had a distal tumor, except for patients 1 and 2 (both middle third) and 18 to 20 (gastroesophageal junction). In graph (A) the peak-to-peak motion is shown. The blue solid lines indicate the mean peak-to-peak amplitude based on the two baseline scans. The estimated short-term ( 45 seconds) and long-term drift $(\sim 10$ minutes $)$ are shown in the (B) and (C), respectively. In both graphs $\mathrm{y}=0$ is indicated with a dotted line and represents the absence of drift.

* The cine-MRI series of time point 4 for this patient were excluded because of table movement that occurred between the cine-MRI series

\section{Long-term intra-fraction motion}

The quantification of shift in CC motion at the start of the drift lines from both the first and second cine-MRI series indicated the presence of drift within the intermediate 10 minutes between the acquisitions of the two series. One time point was excluded due to a table movement between the 2 series. A mean long-term drift (absolute values) of $1.5 \mathrm{~mm}$ (SD 1.8) was found, with a maximum long-term drift of $11.6 \mathrm{~mm}$. For each patient the long-term drift is visualized for all 6 time-points in Figure 4c. 


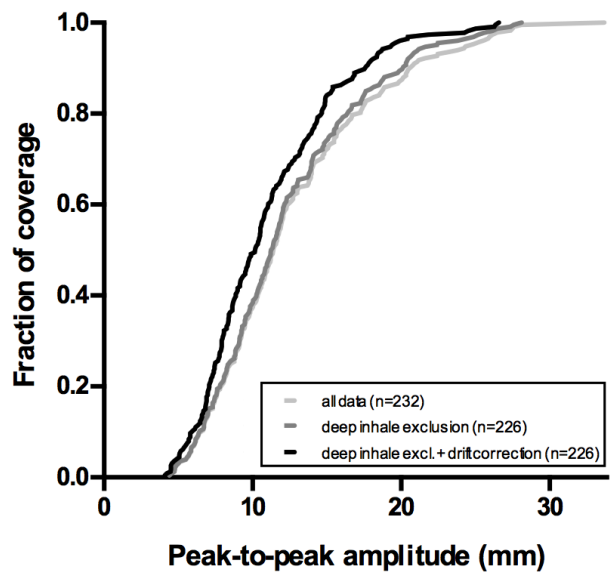

Figure 5. Fraction of scans with a peak-to-peak amplitude lower than the indicated peak-to-peak amplitude, both for the original dataset $(n=232)$, the dataset after exclusion of deep inhales $(n=226)$, and the dataset corrected for tumor drift $(n=226)$. The shift to the left in the lines visualizes the decrease in average peak-to-peak amplitudes after drift correction.

\section{Discussion}

In this study, individual intra-fraction motion of esophageal tumors was weekly monitored during the course of nCRT. Interestingly, it was shown that the motion amplitude varied considerably between and within patients. Furthermore, it was found that a baseline scan is not representative to predict tumor motion throughout treatment. Similarly to other studies, it was found that the largest tumor motion occurred in the CC direction.

The large variations in CC amplitudes could be explained by tumor drift within cine-MRI series (short-term drift), but also by a more constant tumor motion pattern that includes a deep inhale, resulting in large peak-to-peak amplitudes. The duration of one fraction of $1.8 \mathrm{~Gy}$ (approximately 2 minutes) is comparable with the length of one cine-MRI series, which makes the tumor motion found in the cine-MRI series representative for motion that could occur during the delivery of one fraction. Furthermore, long-term drift that occurred within a 10-minute time-interval was quantified, since this could be representative for tumor drift in a time-interval needed for daily dose optimization of the dose plan between positioning of the patient and the actual start of radiation delivery. The radiation dose distributions of patients with large tumor motion have been shown to be influenced by this tumor motion. ${ }^{21,22}$ Future work will concentrate on the possible dosimetric consequences of tumor motion for radiotherapy delivery in esophageal cancer and the potential of using personalized motion margins. Furthermore, strategies to mitigate the effect of tumor motion deserve further investigation. 
Some previous studies have mentioned pre-treatment identification of regular and irregular breathers. ${ }^{23}$ A patient-specific tumor margin for the delivery of radiotherapy could then be determined based on 1 or 2 initial cine-MRI series. However, this is contradicted by the finding of the current study, since large variation in intra-fraction motion is observed in some patients, indicating that an estimation of tumor motion prior to the start of treatment may not represent the actual tumor motion during treatment. The application of fixed margins for esophageal tumors during radiotherapy, e.g. using the well-known 'margin recipe ${ }^{24}$, might not be sufficient when taking the complexity and large variety of tumor motion found in this study into account. However, knowledge of the tumor motion during radiotherapy could enable more conformal dose delivery and individualized treatment strategies. Currently available systems in which tumor tracking during dose delivery is possible are the CyberKnife, using X-ray imaging ${ }^{25}$, and the Calypso, using several internal markers for tumor tracking. ${ }^{26}$ Furthermore, an integrated MRI and Linac provides the advantages of an MRI system, enabling precise tracking of both tumor and surrounding soft tissues during dose delivery. ${ }^{12-15}$ Ultimately, motion margins and consequently unnecessary radiation dose to surrounding organs at risk can be reduced by these approaches.

The conclusion of this study differs from that of Jin et al. ${ }^{27}$, who state that motion defined in pre-treatment $4 \mathrm{DCT}$ is representative for motion throughout treatment. Furthermore, they stated that $97 \%$ of the cases had a motion amplitude of less than $10 \mathrm{~mm}$. While we observed that, after drift correction, only $49 \%$ showed a peak-to-peak amplitude of less than $10 \mathrm{~mm}$. Several differences in study setup can explain this. Firstly, both studies report on relatively small patient groups. In our study all patients were male and mainly distal tumors were included, while the study of Jin et al. includes several females and shows a larger variety in tumor locations. Especially the more proximally located tumors exhibit the smallest motion ${ }^{11}$ and variability ${ }^{27}$, which explains the smaller overall reported variability. Furthermore, tumor motion between males and females could also differ, based on anatomical variations of the thorax or a different breathing pattern..$^{28,29}$ Secondly, 4D(CB) CT reconstruction might obscure tumor drift and large inhales, which could be the origin of the larger variability in intra-fraction motion observed in some patients in our MR-based study.

Some limitations apply to our study. First, our study uses temporal 2D datasets, which could lead to the presence of some out-of-plane motion. The analysis was performed on a coronal slices, so out-of-plane motion would be in the AP direction. The average motion observed in this plane is $3.8 \mathrm{~mm}$, which is below the scanned slice thickness of $5 \mathrm{~mm}$. However, the difference in CC motion found between the coronal and sagittal scans hints to the presence of some out-of-plane motion, which could result in an underestimation or overestimation of tumor motion. Second, cine-MRI series were acquired with both arms positioned next 
to the body, this could have influenced the reported tumor motion amplitude when compared to the currently used radiotherapy position where both arms are raised above the head. However, the body positioning was consequent throughout all cine-MRI series. Furthermore, this positioning is not expected to influence the main findings of the current study regarding the non-representativeness of a baseline scan for tumor motion throughout treatment and varying motion amplitudes between and within patients.

The size of the motion found in 20 esophageal tumors in 3 different directions presented in this study compares well with previously published work in esophageal cancer. ${ }^{11,30}$ Previous published work also described correlations between clinical tumor stage and the mobility of the esophageal tumor. A significantly smaller tumor motion was found in CT4 tumors compared to cT1-T3 tumors, possibly due to the fact that cT4 tumors have an increased adhesion to surrounding tissues. ${ }^{31}$ In the current study no statistical analyses were performed to assess the association between tumor location ${ }^{11}$ or clinical tumor stage $e^{31}$ and tumor motion due to the relatively small sample size including mainly distal tumors. In a larger, more heterogeneous cohort, the relation and clinical characteristics could be studied more extensively.

In conclusion, the largest esophageal tumor motion prior to, and throughout the course of nCRT, was found in CC direction, followed by the AP and LR direction using cine-MRI series. Tumor motion in CC direction is highly variable between and within patients during the course of nCRT, as well as within one cine-MRI series (45 seconds) and over a 10-minute interval. It was shown that esophageal tumor motion does not only comprise of respiratory motion, but is also caused by a tumor drift in some patients. The stochastic nature of both deep inhales and tumor drift indicates that real-time tumor motion management during radiotherapy is a prerequisite to safely reduce treatment margins. 


\section{References}

1. van Hagen P, Hulshof MCCM, van Lanschot JJB, et al. Preoperative chemoradiotherapy for esophageal or junctional cancer. $N$ Engl J Med. 2012;366:2074-84.

2. Donahue JM, Nichols FC, Li Z, et al. Complete pathologic response after neoadjuvant chemoradiotherapy for esophageal cancer is associated with enhanced survival. Ann Thorac Surg. 2009;87:392-9.

3. Berger AC, Farma J, Scott WJ, et al. Complete response to neoadjuvant chemoradiotherapy in esophageal carcinoma is associated with significantly improved survival. J Clin Oncol. 2005;23:4330-4337.

4. Yamashita H, Kida S, Sakumi A, et al. Four-Dimensional Measurement of the Displacement of Internal Fiducial Markers During 320-Multislice Computed Tomography Scanning of Thoracic Esophageal Cancer. Int J Radiat Oncol. 2011;79:588-595.

5. Patel AA, Wolfgang JA, Niemierko A, et al. Implications of Respiratory Motion as Measured by FourDimensional Computed Tomography for Radiation Treatment Planning of Esophageal Cancer. Int J Radiat Oncol Biol Phys. 2009;74:290-296.

6. Dieleman EMT, Senan S, Vincent A, et al. Four-dimensional computed tomographic analysis of esophageal mobility during normal respiration. Int J Radiat Oncol Biol Phys. 2007;67:775-780.

7. Yaremko BP, Guerrero TM, McAleer MF, et al. Determination of Respiratory Motion for Distal Esophagus Cancer Using Four-Dimensional Computed Tomography. Int J Radiat Oncol Biol Phys. 2008;70:145-153.

8. Feng M, Balter JM, Normolle D, et al. Characterization of Pancreatic Tumor Motion Using Cine MRI: Surrogates for Tumor Position Should Be Used With Caution. Int J Radiat Oncol. 2009;74:884-891.

9. Park S, Farah R, Shea SM, et al. Evaluation of lung tumor motion management in radiation therapy with dynamic MRI. Proc SPIE Int Soc Opt Eng. 2017;10135:101351R.

10. Shi X, Diwanji T, Mooney KE, et al. Evaluation of template matching for tumor motion management with cine-MR images in lung cancer patients. Med Phys. 2014;41:52304.

11. Lever FM, Lips IM, Crijns SPM, et al. Quantification of Esophageal Tumor Motion on Cine-Magnetic Resonance Imaging. Int J Radiat Oncol Biol Phys. 2013;88(2):419-424.

12. Kontaxis C, Bol GH, Lagendijk JJW, Raaymakers BW. A new methodology for inter- and intrafraction plan adaptation for the MR-linac. Phys Med Biol. 2015;60:7485-7497.

13. Kontaxis C, Bol GH, Stemkens B, et al. Towards fast online intrafraction replanning for free-breathing stereotactic body radiation therapy with the MR-linac. Phys Med Biol. 2017;62:7233-7248.

14. Lagendijk JJW, Raaymakers BW, Raaijmakers AJE, et al. MRI/linac integration. Radiother Oncol. 2008;86:25-29.

15. Mutic S, Dempsey JF. The ViewRay System: Magnetic Resonance-Guided and Controlled Radiotherapy. Semin Radiat Oncol. 2014;24:196-199.

16. van Rossum PSN, van Lier ALHMW, van Vulpen M, et al. Diffusion-weighted magnetic resonance imaging for the prediction of pathologic response to neoadjuvant chemoradiotherapy in esophageal cancer. Radiother Oncol. 2015;115:163-170.

17. Yushkevich PA, Piven J, Hazlett HC, et al. User-guided 3D active contour segmentation of anatomical structures: Significantly improved efficiency and reliability. Neuroimage. 2006;31:1116-1128.

18. Klein S, Staring M, Murphy K, et al. Elastix: A Toolbox for Intensity-Based Medical Image Registration. IEEE Trans Med Imaging. 2010;29:196-205.

19. Zachiu C, Denis de Senneville B, Moonen C, Ries M. A framework for the correction of slow physiological drifts during MR-guided HIFU therapies: Proof of concept. Med Phys. 2015;42:4137-4148.

20. Roujol S, Ries M, Moonen C, de Senneville BD. Automatic Nonrigid Calibration of Image Registration for Real Time MR-Guided HIFU Ablations of Mobile Organs. IEEE Trans Med Imaging. 2011;30:1737-1745.

21. Trofimov A, Vrancic C, Chan TCY, et al. Tumor trailing strategy for intensity-modulated radiation therapy of moving targets. Med Phys. 2008;35:1718-1733.

22. Tang S, Deville C, McDonough J, et al. Effect of intrafraction prostate motion on proton pencil beam scanning delivery: A quantitative assessment. Int J Radiat Oncol Biol Phys. 2013;87:375-382.

23. Chen X, Lu H, Tai A, et al. Determination of Internal Target Volume for Radiation Treatment Planning of Esophageal Cancer by Using 4-Dimensional Computed Tomography (4DCT). Int J Radiat Oncol. 2014;90:102-109. 
24. van Herk M, Remeijer P, Rasch C, Lebesque JV. The probability of correct target dosage: dose-population histograms for deriving treatment margins in radiotherapy. Int J Radiat Oncol. 2000;47:1121-1135.

25. Coste-Manière E, Olender D, Kilby W, Schulz RA. Robotic whole body stereotactic radiosurgery: clinical advantages of the Cyberknife integrated system. Int J Med Robot. 2005;1:28-39.

26. Bell LJ, Eade T, Kneebone A, et al. Initial experience with intra-fraction motion monitoring using Calypso guided volumetric modulated arc therapy for definitive prostate cancer treatment. J Med Radiat Sci. 2017;64:25-34.

27. Jin P, Hulshof MCCM, van Wieringen N, et al. Interfractional variability of respiration-induced esophageal tumor motion quantified using fiducial markers and four-dimensional cone-beam computed tomography. Radiother Oncol. 2017;124:147-154.

28. Ragnarsdóttir M, Kristinsdóttir EK. Breathing Movements and Breathing Patterns among Healthy Men and Women 20-69 Years of Age. Respiration. 2006;73:48-54.

29. Parreira VF, Bueno CJ, França DC, et al. Padrão respiratório e movimento toracoabdominal em indivíduos saudáveis: influência da idade e do sexo. Rev Bras Fisioter. 2010;14:411-416.

30. Jin P, Hulshof MCCM, de Jong R, et al. Quantification of respiration-induced esophageal tumor motion using fiducial markers and four-dimensional computed tomography. Radiother Oncol. 2016;118:492-497.

31. Kobayashi Y, Myojin M, Shimizu S, Hosokawa M. Esophageal motion characteristics in thoracic esophageal cancer: Impact of clinical stage T4 versus stages T1-T3. Adv Radiat Oncol. 2016;1:222-229. 


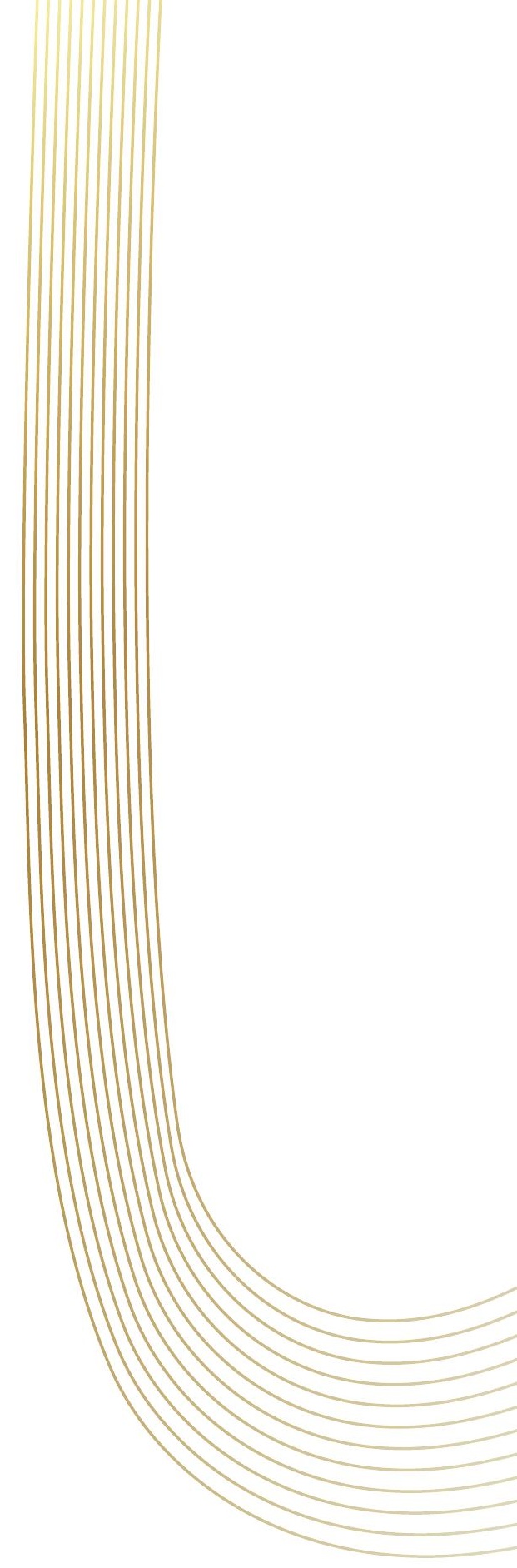


Chapter 4 Radiation dose and pathological response in
oesophageal cancer patients treated with neoadjuvant
chemoradiotherapy followed by surgery:
a multi-institutional analysis Acta Oncologica. 2019;58(10):1358-1365.

A.S. Borggreve ${ }^{\star}$, M. Thomas ${ }^{\star}$, P.S.N. van Rossum, C. Perneel, J. Moons, E. van Daele, R. van Hillegersberg, W. Deng, P. Pattyn, S. Mook, T. Boterberg, J.P. Ruurda, P. Nafteux, S.H. Lin*, K. Haustermans*

${ }^{*}$ Joint first and last authorship. 


\section{Abstract}

\section{Purpose}

To explore whether a higher neoadjuvant radiation dose increases the probability of a pathological complete ( $\mathrm{pCR}$ ) or major ( $\mathrm{pMR}$ ) response in oesophageal cancer patients.

\section{Materials and methods}

Between 2000-2017, 1048 patients from four institutions were stratified according to prescribed neoadjuvant radiation doses of $36.0 \mathrm{~Gy}$ (13.3\%), $40.0 \mathrm{~Gy}$ (7.4\%), $41.4 \mathrm{~Gy}$ (20.1\%), $45.0 \mathrm{~Gy}(25.5 \%)$ or $50.4 \mathrm{~Gy}(33.7 \%)$ in 1.8-2.0 Gy fractions. Endpoints were pCR (tumour regression grade (TRG) 1) and pMR (TRG $1+2$ ). Multivariable binary (TRG $1+2$ vs. TRG $>2$ ) and ordinal (TRG 1 vs. TRG 2 vs. TRG >2) logistic regression analyses were performed, with subgroup analyses according to histology (squamous cell carcinoma (SCC) vs. adenocarcinoma (AC)). Variables entered in the regression model along with neoadjuvant radiation dose were clinical tumour stage (cT), histology, chemotherapy regimen, induction chemotherapy and time from neoadjuvant chemoradiation to surgery.

\section{Results}

A pCR was observed in 312 patients (29.8\%), of whom $22.7 \%$ patients with AC and $49.6 \%$ with SCC. No radiation dose response relation was observed for pCR (OR: 1.01, 95\% CI: 0.98-1.05 for AC and OR: 1.03, 95\% CI: 0.96-1.10 for SCC). A pMR was observed in 597 patients (57.0\%); in 53.4\% patients with AC and in 67.2\% patients with SCC. A higher radiation dose increased the probability of achieving pMR (OR: 1.04, 95\% CI: 1.02-1.05). Factors reducing this probability were advanced $\mathrm{cT}$ stage (reference $=\mathrm{cT} 1-2$; $\mathrm{cT} 3: \mathrm{OR}: 0.54$, 95\% CI: 0.37-0.80; cT4: OR: 0.45, 95\% CI: 0.24-0.84), AC histology (reference = SCC; OR: $0.62,95 \%$ CI: 0.44-0.88), the use of non-platinum based chemotherapy in SCC patients (OR: $0.30,95 \%$ CI: $0.10-0.91$ ) and platinum based chemotherapy without induction chemotherapy in patients with AC (OR: 0.56, 95\% CI: 0.42-0.76). The radiation dose response relation was confirmed in a subgroup analysis of histologic subtypes (OR: 1.02, 95\% CI: $1.01-1.04$ for AC and OR: 1.05, 95\% CI: 1.02-1.08 for SCC).

\section{Conclusions}

Neoadjuvant radiation dose impacts pathological response in terms of pMR in oesophageal cancer patients. No radiation dose response effect was observed for $\mathrm{pCR}$. Further prospective trials are needed to investigate the dose response relation in terms of pCR. 


\section{Introduction}

Neoadjuvant chemoradiotherapy (nCRT) for locally advanced oesophageal cancer has been shown to improve locoregional control and survival compared to surgery alone in several randomized controlled trials..$^{1-3}$ These results have led to the widespread adoption of trimodality therapy as standard of care for locally advanced oesophageal cancer, consisting of preoperative chemotherapy with concurrent radiotherapy followed by oesophagectomy. ${ }^{4,5}$ The improvement in survival after trimodality therapy is largely dependent on tumour response to nCRT, which can be assessed by histological examination of the resected oesophagus. Overall, a pathological complete response (pCR), being defined as complete absence of viable tumour cells in the resection specimen, is obtained in $16-40 \%$ of patients. ${ }^{13,6}$ This wide range in $\mathrm{pCR}$ rates might be due to considerable heterogeneity in preoperative treatment regimens, with neoadjuvant radiation doses ranging from $36.0 \mathrm{~Gy}$ to $50.4 \mathrm{~Gy}$, accompanied by various chemotherapy regimens. ${ }^{1-3,6}$ In oesophageal cancer, the use of nCRT mainly aims at pCR. However, it could be argued that patients with a complete and near-complete response might be clustered and described as pathological major response (pMR). ${ }^{7}$ This is a common approach for response assessment in rectal cancer patients, especially since the interval between nCRT and surgery can be heterogeneous and might impact response rates. ${ }^{8-12}$

A possible strategy for treatment intensification could be to increase the neoadjuvant radiation dose. However, higher radiation doses to the tumour also lead to higher doses to the surrounding organs at risk (OAR) and have been shown to increase postoperative complication rates. ${ }^{13-18}$ On the other hand, the increase in possibilities with proton therapy and the introduction of MRI guided radiation delivery, make it feasible to escalate the radiation dose without increasing the dose to OAR. ${ }^{19-21}$ The available studies that assess whether neoadjuvant radiation dose influences tumour response rates in patients with oesophageal cancer are equivocal, and the debate regarding the optimal neoadjuvant radiation dose is still ongoing. ${ }^{22-25}$ Therefore, the goal of the current study was to retrospectively explore the hypothesis that a higher neoadjuvant radiation dose could increase pathological tumour response rates in oesophageal cancer patients, in terms of achievement of $\mathrm{pCR}$ and $\mathrm{pMR}$.

\section{Materials and methods}

\section{Patients}

Consecutive patients with locally advanced resectable oesophageal cancer scheduled for and treated with nCRT followed by surgery at four high volume institutes between 2000 and 2017 were included. Patients were stratified according to the prescribed total neoadjuvant radiation dose of $36.0 \mathrm{~Gy}, 40.0 \mathrm{~Gy}, 41.4 \mathrm{~Gy}, 45.0 \mathrm{~Gy}$ and $50.4 \mathrm{~Gy}$. Exclusion 
criteria were (1) a histologic subtype other than squamous cell carcinoma (SCC) or adenocarcinoma (AC) as based on the resection specimen and in case of a pCR based on the primary tumour biopsy, (2) the presence of distant metastases and (3) treatment with proton radiotherapy. Patients were identified from prospectively maintained databases in the following four university medical centres: University Hospitals Leuven (Belgium), University Medical Center Utrecht (the Netherlands), University of Texas MD Anderson Cancer Center (United States of America) and Ghent University Hospital (Belgium). All patients underwent an endoscopy with biopsy for histological proof of malignancy and a computed tomography (CT) scan of the chest and abdomen or a ${ }^{18} \mathrm{~F}$-fluorodeoxyglucose positron emission tomography with integrated CT $\left({ }^{18} \mathrm{~F}-\mathrm{FDG}-\mathrm{PET} / \mathrm{CT}\right)$ for staging. Other examinations were performed according to institutional guidelines.

\section{Treatment}

Radiotherapy was delivered in fractions of $1.8 \mathrm{~Gy}$ or $2.0 \mathrm{~Gy}$ by three-dimensional conformal radiotherapy (3D-CRT), intensity modulated radiotherapy (IMRT) or volumetric arc therapy (VMAT). Delineation of the target volume was according to institutional guidelines. Typically gross target volume (GTV) was based on endoscopy, CT or ${ }^{18} \mathrm{~F}-\mathrm{FDG}-\mathrm{PET} / \mathrm{CT}$ and expanded with a $3-4 \mathrm{~cm}$ margin in cranio-caudal direction and $0.5-1 \mathrm{~cm}$ circumferentially, respecting the anatomic boundaries. The choice of chemotherapy regimen and whether or not induction chemotherapy prior to nCRT was administered, was at the discretion of the treating multidisciplinary team. The patients receiving 36.0 Gy were treated in a single hospital and generally received platinum based chemotherapy without induction therapy. In the single hospital treating patients with $41.4 \mathrm{~Gy}$, only platinum based chemotherapy and no induction therapy was given (as per CROSS trial). ${ }^{3}$ One hospital treated patients with both $40.0 \mathrm{~Gy}$ and $45.0 \mathrm{~Gy}$ and all patients received platinum based chemotherapy, most commonly combined with induction chemotherapy. The fourth hospital treated patients with 45.0 Gy and 50.4 Gy with either a platinum or non-platinum based chemotherapy regimen, and sometimes administered induction chemotherapy. After nCRT, patients were scheduled for transthoracic or transhiatal surgery with two- or three-field lymphadenectomy, based on the location of the tumour and pathological lymph nodes, as well as the preference of the surgeon.

\section{Outcome measures}

Clinical and treatment-related characteristics were acquired from the prospectively collected databases at the four centres. The time from nCRT to surgery was defined as the interval in days between the last day of radiotherapy and the date of surgery. Histologic tumour regression grades (TRG) of the primary tumour were based on histopathological evaluation of the resection specimen by dedicated gastrointestinal pathologists and grouped into three 
TRG groups: TRG 1 defined as Mandard 1 or $0.0 \%$ viable tumour cells (pCR), TRG 2 defined as Mandard 2 or $<10.0 \%$ viable tumour cells and TRG $>2$ defined as Mandard $>2$ or $>10.0 \%$ viable tumour cells. ${ }^{26,27}$ pMR was defined as TRG 1 and 2 combined.

Overall survival was calculated based from the day of surgery to the date of all-cause death or last day of follow-up.

\section{Statistical analysis}

Summary statistics were presented as medians and interquartile range (IQR) for continuous variables, and as frequencies and percentages for categorical variables. To evaluate group differences in patient and treatment variables, the chi-square and ANOVA test were used for categorical and continuous variables, respectively.

The relation between neoadjuvant radiation dose and pathological response was studied in a multivariable binary and ordinal logistic regression analysis. The ordinal logistic regression analysis considered three categories of the dependent variable: TRG $>2$, TRG 2 and TRG 1 (i.e. pCR). In the multivariable binary logistic regression model, the dependent variable was categorised as TRG 1 and 2 (i.e. pMR) versus TRG $>2$. Variables to be entered in the regression model along with neoadjuvant radiation dose were based on clinical relevance and literature review of potential confounders: clinical tumour stage (cT), histology, chemotherapy regimen, the use of induction chemotherapy before nCRT, and the time from nCRT to surgery. Furthermore, interactions between histology and (induction) chemotherapy were added to the models when appropriate. Pre-specified subgroup analyses by histology were performed. Patients with missing outcome or covariables were excluded from the analyses.

The Kaplan-Meier method was used to estimate overall survival of pCR and pMR in the entire patient cohort and according to histology.

All analyses were performed with SPSS version 25.0 (IBM Corp., Armonk, NY, USA).

\section{Results}

\section{Patients}

A total of 1102 patients were retrieved from the databases. Fifty-four patients (45 patients with AC and 9 patients with SCC) with a missing endpoint or covariable were excluded, resulting in 1048 patients eligible for analyses (Supplementary figure 1). Patients received either 36.0 Gy (139, 13.3\%), 40.0 Gy (78, 7.4\%), 41.4 Gy (211, 20.1\%), 45.0 Gy (267, 25.5\%) or $50.4 \mathrm{~Gy}(353,33.7 \%)$. Patient, tumour and treatment-related characteristics of the patient cohort are presented in Table 1 . The majority of patients were male $(837,79.9 \%)$ and the median age was 62 years (IQR 55-68 years). The predominant histologic tumour type was 
AC $(774,73.9 \%)$ and cT3 was the most common tumour stage (857, 81.8\%). Most patients had positive lymph nodes upon clinical staging (796, 76.0\%). The patients treated with 36.0 Gy received platinum based chemotherapy (except one) without induction therapy (except one). In one hospital treating patients with $45.0 \mathrm{~Gy}$ and $50.4 \mathrm{~Gy}$, a non-platinum based chemotherapy regimen was administered in half of the cases and induction chemotherapy was given in $38.3 \%$ of all patients.

Table 1. Baseline characteristics of the study population

\begin{tabular}{|c|c|c|c|c|c|c|c|}
\hline Characteristic & $\begin{array}{l}\text { Total } \\
\mathrm{n}=1048 \\
(100 \%)\end{array}$ & $\begin{array}{c}36.0 \mathrm{~Gy} \\
\mathrm{n}=139 \\
(13.3 \%)\end{array}$ & $\begin{array}{l}40.0 \mathrm{~Gy} \\
\mathrm{n}=78 \\
(7.4 \%)\end{array}$ & $\begin{array}{c}41.4 \mathrm{~Gy} \\
\mathrm{n}=211 \\
(20.1 \%)\end{array}$ & $\begin{array}{c}45.0 \mathrm{~Gy} \\
\mathrm{n}=267 \\
(25.5 \%)\end{array}$ & $\begin{array}{l}50.4 \mathrm{~Gy} \\
\mathbf{n}=353 \\
(33.7 \%)\end{array}$ & p-value \\
\hline $\begin{array}{l}\text { Age at diagnosis, years } \\
\text { (median (IQR)) }\end{array}$ & $62(55-68)$ & $62(55-69)$ & $60(53-66)$ & $67(60-71)$ & $62(55-69)$ & $60(52-65)$ & $<0.001$ \\
\hline \multicolumn{8}{|l|}{ Sex } \\
\hline Male & $837(79.9)$ & $112(80.6)$ & $58(74.4)$ & $160(75.8)$ & $196(73.4)$ & $311(88.1)$ & $<0.001$ \\
\hline Female & $211(20.1)$ & $27(19.4)$ & $20(25.6)$ & $51(24.2)$ & $71(26.6)$ & $42(11.9)$ & \\
\hline \multicolumn{8}{|l|}{ Chemotherapy regimen } \\
\hline Platinum based & $852(81.3)$ & $138(99.3)$ & $78(100)$ & $211(100)$ & $246(92.1)$ & $179(50.7)$ & $<0.001$ \\
\hline Non-platinum based & $196(18.7)$ & $1(0.7)$ & $0(0.0)$ & $0(0.0)$ & $21(7.9)$ & $174(49.3)$ & \\
\hline \multicolumn{8}{|l|}{ Induction chemotherapy } \\
\hline Yes & $420(40.1)$ & $1(0.7)$ & $61(78.2)$ & $0(0.0)$ & $235(88.0)$ & $123(34.8)$ & $<0.001$ \\
\hline No & $628(59.9)$ & $138(99.3)$ & $17(21.8)$ & $211(100)$ & $32(12.0)$ & $230(65.2)$ & \\
\hline $\begin{array}{l}\text { Days end radiation until } \\
\text { surgery (median (IQR)) }\end{array}$ & $53(44-64)$ & $34(29-38)$ & $55(47-64)$ & $62(50-75)$ & $55(47-64)$ & $57(47-78)$ & $<0.001$ \\
\hline \multicolumn{8}{|l|}{ Clinical T status } \\
\hline $\mathrm{T} 1$ & $8(0.8)$ & $2(1.4)$ & $0(0.0)$ & $3(1.4)$ & $1(0.4)$ & $2(0.6)$ & $<0.001$ \\
\hline $\mathrm{T} 2$ & $126(12.0)$ & $15(10.8)$ & $6(7.7)$ & $36(17.1)$ & $31(11.6)$ & $38(10.8)$ & \\
\hline T3 & $857(81.8)$ & $117(84.2)$ & $46(59.0)$ & $169(80.1)$ & $218(81.6)$ & $307(87.0)$ & \\
\hline $\mathrm{T} 4$ & $57(5.4)$ & $5(3.6)$ & $26(33.3)$ & $3(1.4)$ & $17(6.4)$ & $6(1.7)$ & \\
\hline \multicolumn{8}{|l|}{ Clinical N status } \\
\hline No & $244(23.3)$ & $20(14.4)$ & $4(5.1)$ & $59(28.0)$ & $37(13.9)$ & $124(35.1)$ & $<0.001$ \\
\hline $\mathrm{N}+$ & $796(76.0)$ & $113(81.3)$ & $73(93.6)$ & $152(72.0)$ & $230(86.1)$ & $228(64.6)$ & \\
\hline $\mathrm{Nx}$ & $8(0.8)$ & $6(4.3)$ & $1(1.3)$ & $0(0.0)$ & $0(0.0)$ & $1(0.3)$ & \\
\hline \multicolumn{8}{|l|}{ Histology } \\
\hline Squamous cell carcinoma & $274(26.1)$ & $52(37.4)$ & $44(56.4)$ & $66(31.3)$ & $81(30.3)$ & $31(8.8)$ & $<0.001$ \\
\hline Adenocarcinoma & $774(73.9)$ & $87(62.6)$ & $34(43.6)$ & $145(68.7)$ & $186(69.7)$ & $322(91.2)$ & \\
\hline \multicolumn{8}{|l|}{ Pathologic response } \\
\hline TRG 1 & $312(29.8)$ & $37(26.6)$ & $26(33.3)$ & $63(29.9)$ & $89(33.3)$ & $97(27.5)$ & NA \\
\hline TRG 2 & $285(27.2)$ & $35(25.2)$ & $20(25.6)$ & $45(21.3)$ & $83(31.1)$ & $102(28.9)$ & \\
\hline TRG $>2$ & $451(43.0)$ & $67(48.2)$ & $32(41.0)$ & $103(48.8)$ & $95(35.6)$ & $154(43.6)$ & \\
\hline
\end{tabular}

$I Q R$ interquartile range, TRG tumour regression grade, $N A$ not applicable (appropriate analyses reported elsewhere)

Values are numbers of patients, with column-based percentages in parentheses unless stated otherwise. 


\section{Pathologic complete response}

A pCR was demonstrated in 312 patients (29.8\%), of which 176 of 774 patients $(22.7 \%)$ with AC and 136 of 274 patients (49.6\%) with SCC. A dose response diagram for the endpoint TRG 1 (pCR) vs. TRG 2 vs. TRG $>2$ is presented in Figure 1.

Multivariable ordinal logistic regression analysis with TRG $>2$, TRG 2 and TRG 1 as outcome categories demonstrated no significant impact of total neoadjuvant radiation dose for both histologic subtypes (per additional Gy: OR 1.01, 95\% CI 0.98-1.05, p=0.403 for AC and OR 1.03, 95\% CI 0.96-1.10, p=0.402 for SCC) (Table 2). In the patients with AC, advanced tumour stage (with cT1-2 as reference; cT3-4: OR 0.53, 95\% CI 0.36-0.78, $\mathrm{p}=0.002$ ) and the use of platinum based chemotherapy without induction chemotherapy (OR 0.56, 95\% CI 0.41-0.77, p<0.001) reduced the odds of pCR. For patients with SCC, only an advanced tumour stage reduced the odds of PCR (with cT1-2 as reference; cT3-4: OR $0.45,95 \%$ CI $0.12-1.11, \mathrm{p}=0.030)$.

\section{Pathologic major response}

A pMR was obtained in 597 of 1048 patients (57.0\%). In binary multivariable logistic regression analysis with $\mathrm{pMR}$ as outcome, the total neoadjuvant radiation dose increased the odds of pMR (per additional Gy: OR 1.04, 95\% CI 1.02-1.05, p<0.001). Factors that reduced these odds were a higher tumour stage (with cT1-2 as reference; cT3: OR 0.54, 95\% CI 0.37-0.80, $\mathrm{p}=0.002$ and cT4: OR 0.45, 95\% CI 0.24-0.84, $\mathrm{p}=0.013$ ) as well as AC histology (OR $0.62,95 \%$ CI $0.44-0.88, \mathrm{p}=0.008$ ). The use of non-platinum based

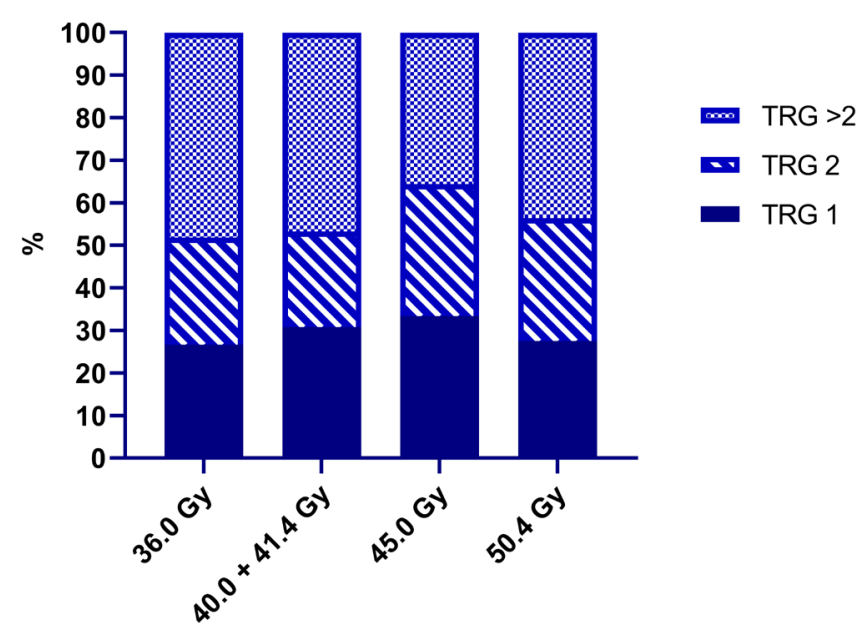

Figure 1. Dose response diagram for tumour regression grades (TRG) within the entire patient cohort 
chemotherapy in patients with SCC decreased the odds of pMR (OR 0.30, 95\% CI: 0.10$0.91, \mathrm{p}=0.034$ ). The use of platinum based chemotherapy without induction chemotherapy in patients with $\mathrm{AC}$ had a negative effect on pMR (OR 0.56, 95\% CI 0.42-0.76, p<0.001) (Table 3).

\section{Subgroup analyses for pathologic major response according to histology}

In patients with $\mathrm{AC}$, a pMR was achieved in 413 of 774 patients (53.4\%). In binary multivariable logistic regression analysis with pMR as outcome, the total neoadjuvant radiation dose increased the odds of pMR (per additional Gy: OR 1.02, 95\% CI 1.01-1.04, $\mathrm{p}<0.001$ ). Factors reducing the odds were advanced tumour stage (with cT1-2 as reference; cT3: OR 0.59, 95\% CI 0.38-0.91, p=0.016 and cT4: OR 0.56, 95\% CI 0.23-1.36, p=0.199) and the use of platinum based chemotherapy without induction chemotherapy (OR 0.54, 95\% CI 0.41-0.72, p<0.001) (Table 3).

For SCC, 184 of 274 patients (67.2\%) achieved a pMR. In multivariable binary logistic regression analysis with $\mathrm{pMR}$ as outcome, the total neoadjuvant radiation dose increased the odds of pMR (per additional Gy: OR 1.05, 95\% CI 1.02-1.08, p=0.001). Factors that reduced these odds were higher tumour stage (with cT1-2 as reference; cT3: OR 0.32, 95\% CI $0.12-0.82, \mathrm{p}=0.018$ and cT4: OR $0.23,95 \%$ CI $0.07-0.71, \mathrm{p}=0.011)$ and the use of nonplatinum based chemotherapy (OR 0.27, 95\% CI 0.09-0.83, p=0.022).

Table 2. Multivariable ordinal logistic regression analysis with three pathological response groups as outcome variable (TRG >2, TRG 2 and TRG 1 (pCR)), according to histologic subgroups

\begin{tabular}{llr}
\hline Adenocarcinoma $(\mathbf{n}=\mathbf{7 7 4})$ & OR (95\% CI) & p-value \\
\hline $\begin{array}{l}\text { Total neoadjuvant radiation dose per Gy } \\
\text { Tumour stage (cT1-2 as reference) }\end{array}$ & $1.01(0.98-1.05)$ & 0.403 \\
$\quad$ cT3-4 & $0.53(0.36-0.78)$ & $\mathbf{0 . 0 0 2}$ \\
Platinum based chemotherapy without induction chemotherapy & $0.56(0.41-0.77)$ & $<\mathbf{0 . 0 0 1}$ \\
Days between neoadjuvant chemoradiotherapy and surgery & $1.00(0.99-1.01)$ & 0.812 \\
\hline Squamous cell carcinoma (n=274) & OR (95\% CI) & $\mathbf{p}$-value \\
\hline Total neoadjuvant radiation dose per Gy & $1.03(0.96-1.10)$ & 0.402 \\
Tumour stage (cT1-2 as reference)* & & $\mathbf{0 . 0 3 0}$ \\
$\quad$ cT3-4 & $0.45(0.12-1.11)$ & 0.076 \\
Non-platinum based chemotherapy & $0.36(0.41-0.77)$ & 0.878 \\
Days between neoadjuvant chemoradiotherapy and surgery & $1.00(0.99-1.01)$ & \\
\hline
\end{tabular}

$C I$ confidence interval, $O R$ odds ratio, $p C R$ pathological complete response, $T R G$ tumour regression grade

* Clinical tumour stages were combined for these subgroups analyses because of small patient numbers per stage. The Test of Parallel Lines had a p-value of 0.051 (degrees of freedom $(\mathrm{df})=4$, adenocarcinoma) and $0.386(\mathrm{df}=4$, squamous cell carcinoma) respectively. 
Table 3. Multivariable logistic regression analysis with pathological major response (pMR) as outcome variable in the entire patient cohort and according to histologic subgroups

\begin{tabular}{|c|c|c|}
\hline Entire cohort $(n=1048)$ & OR $(95 \% \mathrm{CI})$ & p-value \\
\hline Total neoadjuvant radiation dose per Gy & $1.04(1.02-1.05)$ & $<0.001$ \\
\hline Adenocarcinoma (squamous cell carcinoma as reference) & $0.62(0.44-0.88)$ & 0.008 \\
\hline \multicolumn{3}{|l|}{ Tumour stage (cT1-2 as reference) $)^{\star}$} \\
\hline cT3 & $0.54(0.37-0.80)$ & 0.002 \\
\hline $\mathrm{cT} 4$ & $0.45(0.24-0.84)$ & 0.013 \\
\hline $\begin{array}{l}\text { Interaction between adenocarcinoma and platinum based } \\
\text { chemotherapy without induction chemotherapy }\end{array}$ & $0.56(0.42-0.76)$ & $<0.001$ \\
\hline $\begin{array}{l}\text { Interaction between squamous cell carcinoma and } \\
\text { non-platinum based chemotherapy }\end{array}$ & $0.30(0.10-0.91)$ & 0.034 \\
\hline Days between neoadjuvant chemoradiotherapy and surgery & $1.00(0.99-1.00)$ & 0.241 \\
\hline Adenocarcinoma $(\mathrm{n}=774)$ & OR (95\% CI) & p-value \\
\hline Total neoadjuvant radiation dose per Gy & $1.02(1.01-1.04)$ & $<0.001$ \\
\hline \multicolumn{3}{|l|}{ Tumour stage (cT1-2 as reference)* } \\
\hline cT3 & $0.59(0.38-0.91)$ & 0.016 \\
\hline cT4 & $0.56(0.23-1.36)$ & 0.199 \\
\hline Platinum based chemotherapy without induction chemotherapy & $0.54(0.41-0.72)$ & $<0.001$ \\
\hline Days between neoadjuvant chemoradiotherapy and surgery & $1.00(0.99-1.00)$ & 0.291 \\
\hline Squamous cell carcinoma $(n=274)$ & OR (95\% CI) & p-value \\
\hline Total neoadjuvant radiation dose per Gy & $1.05(1.02-1.08)$ & 0.001 \\
\hline \multicolumn{3}{|l|}{ Tumour stage (cT1-2 as reference) $)^{\star}$} \\
\hline cT3 & $0.32(0.12-0.82)$ & 0.018 \\
\hline cT4 & $0.23(0.07-0.71)$ & 0.011 \\
\hline Non-platinum based chemotherapy & $0.27(0.09-0.83)$ & 0.022 \\
\hline Days between neoadjuvant chemoradiotherapy and surgery & $1.00(0.98-1.01)$ & 0.674 \\
\hline
\end{tabular}

$C I$ confidence interval, $O R$ odds ratio, $p M R$ pathological major response

${ }^{*} \mathrm{cT} 1$ and $\mathrm{cT} 2$ were combined as a reference category, since only few patients had a cT1 stage.

The Hosmer-Lemeshow goodness of fit test had a p-value of 0.399 (degrees of freedom $(\mathrm{df})=8$, entire patient cohort), $0.754(\mathrm{df}=8$, adenocarcinoma $)$ and $0.276(\mathrm{df}=8$, squamous cell carcinoma) respectively.

\section{Survival analysis}

Median follow-up time of the patients was 25.1 months (IQR 11.0-54.7 months). The median OS of patients with a pCR, TRG 2 and TRG $>2$ was 78 months, 46 months and 28 months, respectively (Supplementary Material 2). For patients with an AC, the median OS was 90 months for pCR, 50 months for TRG 2 and 30 months TRG $>2$. For patients with an SCC, the median OS was 67 months for pCR, 38 months for TRG 2 and 16 months TRG $>2$ (Supplementary Material 2).

The median OS of patients with a pMR and TRG $>2$ was 61 months and 28 months, respectively (Figure 2). For patients with an AC, the median OS was 64 months for pMR and 30 months TRG $>2$. For patients with an SCC patients, the median OS was 55 months for $\mathrm{pMR}$ and 16 months TRG $>2$ (Figure 2). 


\section{A. Entire cohort}

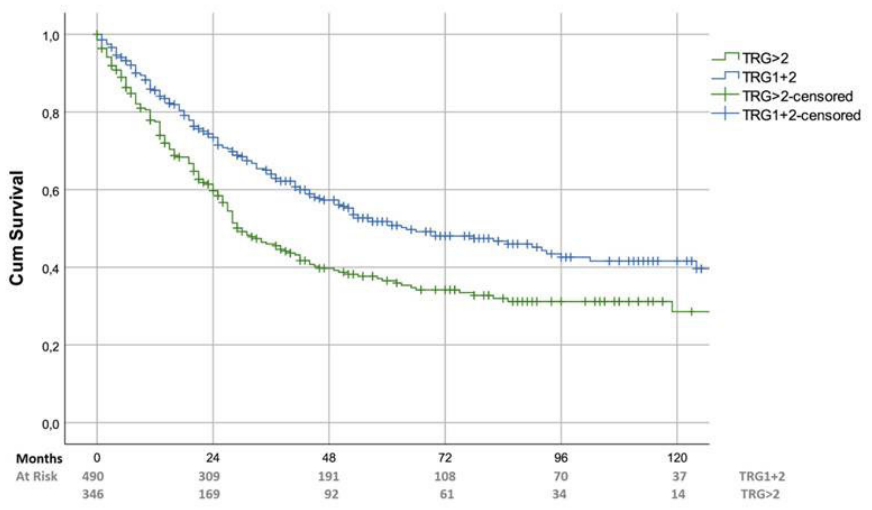

\section{B. Adenocarcinoma}

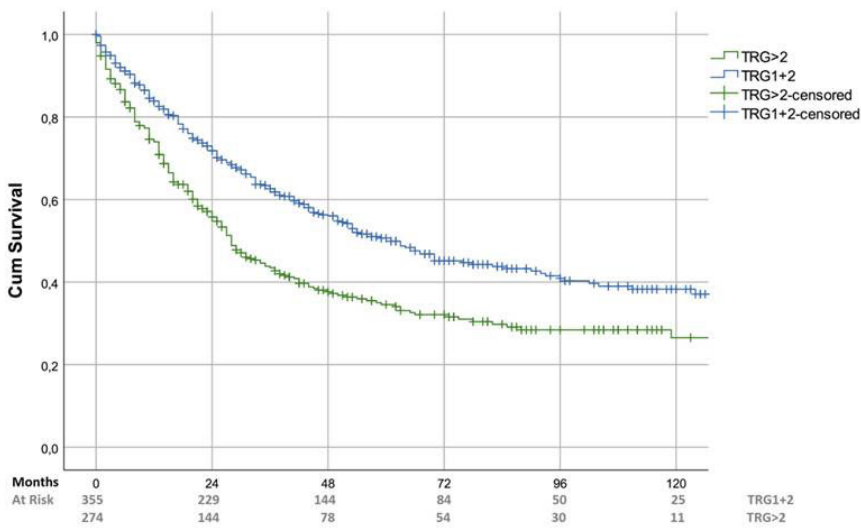

\section{Squamous cell carcinoma}

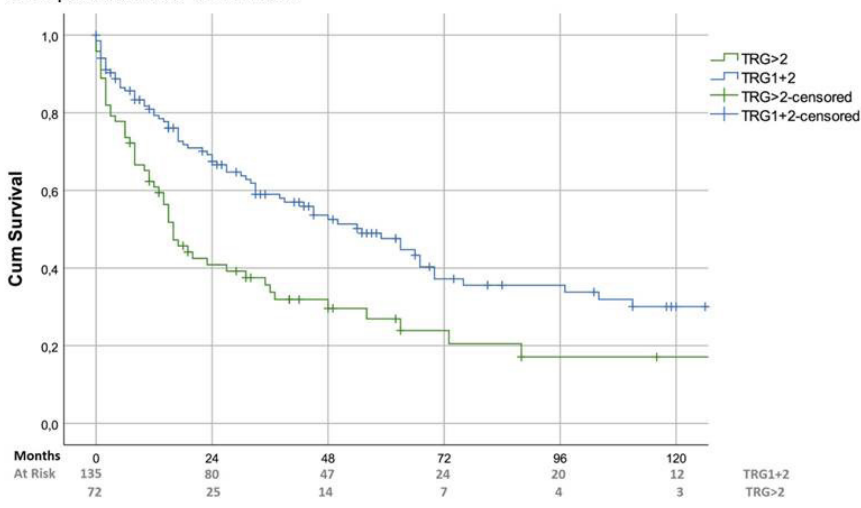

Figure 2. Overall survival curves of tumour regression grades (TRG) 1+2 (pathologic major response; pMR, blue curve) vs. TRG $>2$ (green curve) in the entire patient cohort (A) and according to histologic subgroups (adenocarcinoma: B, squamous cell carcinoma: $\mathbf{C}$ ) 


\section{Discussion}

In this study, we analysed the pathological response rate after nCRT followed by surgery in 1048 patients with oesophageal cancer from four high volume institutes. The balance between the pathological response and the toxicity of neoadjuvant therapy emphasizes the need to explore a dose response relationship, especially in the era of an increasing use of a wait-and-see policy in clinical complete responders. Some evidence of a dose response relation has been described in observational studies. ${ }^{22,25}$ To our knowledge, no randomised controlled trials have been performed comparing different neoadjuvant radiotherapy schedules for patients with oesophageal cancer. Our study did not observe a dose response relation for pCR (TRG 1) in multivariable ordinal regression analyses. However, a dose response relation was observed for radiation dose and pMR (TRG 1+2), where the neoadjuvant radiation dose, besides the tumour stage, histology, induction chemotherapy and the chemotherapy regimen, increased the odds of a pMR. As the cohort of patients with SCC was rather small compared to that of the patients with AC, especially in the subgroup of non-platinum based chemotherapy and in the group of patients receiving 50.4 Gy, the effect of dose in patients with SCC should be interpreted with caution.

Next to the neoadjuvant radiation dose, the impact of the concurrent chemotherapy regimen and the use of induction chemotherapy was studied. A non-platinum based chemotherapy was less effective to achieve a pMR in patients with SCC. When induction chemotherapy was administered in patients with $\mathrm{AC}$, there was an improvement in pMR only when platinum based chemotherapy was used concomitantly. In literature, limited data are available on the use of non-platinum based chemotherapy and the pathological response rate in patients with SCC and AC separately in the preoperative setting in oesophageal cancer patients. ${ }^{28,29}$ The observed differences in chemo- and radiotherapy sensitivity underline the necessity for researching individualized treatment regimens for both histologic subgroups.

Next to pCR, pMR was chosen as secondary outcome variable for several reasons. First, the interval between the last day of radiotherapy and the surgery might affect the degree of pathological response, i.e. a shorter interval between nCRT and surgery might underestimate the presence of a pathological response..$^{30}$ To eliminate this bias, the interval between $\mathrm{nCRT}$ and surgery was taken into account in the multivariable analyses, and pathological complete and near-complete responders were grouped as patients with pMR in a secondary analysis as well. Second, the benefit in overall survival and progression free survival has been suggested to be comparable between patients with a pCR and those with a low percentage of residual tumour cells in a meta-analysis. ${ }^{8}$ Lastly, a similar strategy has been applied in other cancer types, such as rectal cancer, where both clinical complete and near-complete responders are considered eligible for an organ-preservation strategy. ${ }^{9}$. 
In our study, reaching a pMR or pCR, compared to TRG $>2$, was as expected associated with a survival advantage, both in the entire patient cohort as in the subgroups according to histology. ${ }^{8,31}$ Despite the large patient cohort, we acknowledge that these analyses were subject to an unbalanced distribution of confounders, related to clinical and treatmentrelated variables.

Potential limitations of this study include its retrospective nature. Additionally, the tumour regression grade was evaluated by centre-specific pathologists and only evaluated the regression grade of the primary tumour and not of the lymph nodes. As a consequence, persistent disease only at the nodal level was possible. ${ }^{32-34}$ Since the lack of adequate tumour regression grade systems for nodes after preoperative treatment and the relatively low percentage of patients in our study with an ypT0N+, the current study focussed on the primary tumour. However, in order to investigate the effect of increased neoadjuvant radiation dose on survival, the response of the primary tumour should be combined with the pathological nodal stage, since the latter is one of the most important determinants of overall survival after nCRT followed by surgery. ${ }^{8,35,36}$ Another limitation involves that all patients scheduled for and actually treated with nCRT followed by surgery (i.e. preoperative intent) were included in the analysis. As such, there might have been a selection bias by excluding patients who started nCRT but did not proceed to surgery, e.g. patients with a clinical complete response in whom an active surveillance strategy was applied. On the other hand, in a few patients surgery was delayed, due to prolonged treatment recovery from nCRT and observation. Additionally, toxicity data were not available and further studies are warranted to investigate the influence of nCRT on toxicity and postoperative complications. Lastly, we acknowledge that the treatment regimen, i.e. radiation dose and concurrent chemotherapy regimen, was directly linked to the centre, which might have resulted in residual confounding. Despite the aforementioned limitations, major strengths of this study include that this is a large multi-centre study, based on high-quality prospectively collected data. In contrast to previous studies, information on the concurrent chemotherapy regimen and on the use of induction chemotherapy was available, which is important in analysing pathological response rates.

In conclusion, this study demonstrated that the total neoadjuvant radiation dose had an impact on pathological response in terms of pMR in patients with oesophageal cancer. However, no radiation dose response effect was observed for pCR. Further prospective trials are needed to investigate the dose response relation in terms of pCR. 


\section{References}

1. Tepper J, Krasna MJ, Niedzwiecki D, et al. Phase III trial of trimodality therapy with cisplatin, fluorouracil, radiotherapy, and surgery compared with surgery alone for esophageal cancer: CALGB 9781. J Clin Oncol. 2008;26:1086-92.

2. Sjoquist KM, Burmeister BH, Smithers BM, et al. Survival after neoadjuvant chemotherapy or chemoradiotherapy for resectable oesophageal carcinoma: An updated meta-analysis. Lancet Oncol. 2011;12:681-92.

3. van Hagen P, Hulshof MCCM, van Lanschot JJB, et al. Preoperative Chemoradiotherapy for Esophageal or Junctional Cancer. N Engl J Med. 2012;366:2074-84.

4. Lagergren J, Smyth E, Cunningham D, Lagergren P. Oesophageal cancer. Lancet. 2017;390:2383-96.

5. Lordick F, Mariette C, Haustermans K, Obermannová R, Arnold D. Oesophageal cancer: ESMO Clinical Practice Guidelines for diagnosis, treatment and follow-up. Ann Oncol. 2016;27:v50-7.

6. Burmeister BH, Smithers BM, Gebski V, et al. Surgery alone versus chemoradiotherapy followed by surgery for resectable cancer of the oesophagus: a randomised controlled phase III trial. Lancet Oncol. 2005;6:65968.

7. Depypere L, Moons J, Lerut T, et al. Neoadjuvant chemoradiation treatment followed by surgery for esophageal cancer: There is much more than the mandard tumor regression score. Acta Chir Belg. 2016;116:149-55.

8. Tomasello G, Petrelli F, Ghidini M, et al. Tumor regression grade and survival after neoadjuvant treatment in gastro-esophageal cancer: A meta-analysis of 17 published studies. Eur J Surg Oncol. 2017;43:1607-16.

9. Hupkens BJP, Maas M, Martens MH, et al. Organ Preservation in Rectal Cancer After Chemoradiation: Should We Extend the Observation Period in Patients with a Clinical Near-Complete Response? Ann Surg Oncol. 2018;25:197-203.

10. Lee A, Wong AT, Schwartz D, Weiner JP, Osborn VW, Schreiber D. Is There a Benefit to Prolonging the Interval Between Neoadjuvant Chemoradiation and Esophagectomy in Esophageal Cancer? Ann Thorac Surg. 2016;102:433-8.

11. Haisley KR, Laird AE, Nabavizadeh N, et al. Association of Intervals Between Neoadjuvant Chemoradiation and Surgical Resection With Pathologic Complete Response and Survival in Patients With Esophageal Cancer. JAMA Surg. 2016;151:e162743.

12. Tie H, He F, Shen J, et al. Prolonged interval between neoadjuvant chemoradiotherapy and esophagectomy does not benefit the outcome in esophageal cancer: a systematic review and meta-analysis. Dis Esophagus. 2018;31:1-9.

13. Walle C Vande, Ceelen WP, Boterberg T, et al. Anastomotic Complications After Ivor Lewis Esophagectomy in Patients Treated With Neoadjuvant Chemoradiation Are Related to Radiation Dose to the Gastric Fundus. Int J Radiat Oncol Biol Phys. 2012;82:e513-9.

14. Hatakenaka M, Yonezawa M, Nonoshita T, et al. Acute cardiac impairment associated with concurrent chemoradiotherapy for esophageal cancer: Magnetic resonance evaluation. Int J Radiat Oncol Biol Phys. 2012;83:e67-73.

15. Beukema JC, van Luijk P, Widder J, Langendijk JA, Muijs CT. Is cardiac toxicity a relevant issue in the radiation treatment of esophageal cancer? Radiother Oncol. 2015;114:85-90.

16. Lee HK, Vaporciyan AA, Cox JD, et al. Postoperative pulmonary complications after preoperative chemoradiation for esophageal carcinoma: correlation with pulmonary dose-volume histogram parameters. Int J Radiat Oncol Biol Phys. 2003;57:1317-22.

17. Wang SL, Liao Z, Vaporciyan AA, et al. Investigation of clinical and dosimetric factors associated with postoperative pulmonary complications in esophageal cancer patients treated with concurrent chemoradiotherapy followed by surgery. Int J Radiat Oncol Biol Phys. 2006;64:692-9.

18. Tucker SL, Liu HH, Wang S, et al. Dose-volume modeling of the risk of postoperative pulmonary complications among esophageal cancer patients treated with concurrent chemoradiotherapy followed by surgery. Int J Radiat Oncol Biol Phys. 2006;66:754-61.

19. Lagendijk JJW, Raaymakers BW, Raaijmakers AJE, et al. MRI/linac integration. Radiother Oncol. 2008;86:25-9. 
20. Welsh J, Gomez D, Palmer MB, et al. Intensity-modulated proton therapy further reduces normal tissue exposure during definitive therapy for locally advanced distal esophageal tumors: A dosimetric study. Int $J$ Radiat Oncol Biol Phys. 2011;81:1336-42.

21. Lin SH, Merrell KW, Shen J, et al. Multi-institutional analysis of radiation modality use and postoperative outcomes of neoadjuvant chemoradiation for esophageal cancer. Radiother Oncol. 2017;123:376-81.

22. Geh JI, Bond SJ, Bentzen SM, Glynne-Jones R. Systematic overview of preoperative (neoadjuvant) chemoradiotherapy trials in oesophageal cancer: Evidence of a radiation and chemotherapy dose response. Radiother Oncol. 2006;78:236-44.

23. Venkat PS, Shridhar R, Naghavi AO, et al. Dose escalated neoadjuvant chemoradiotherapy with dosepainting intensity-modulated radiation therapy and improved pathologic complete response in locally advanced esophageal cancer. Dis Esophagus. 2017;30:1-9.

24. Ji KSY, Thomas SM, Roman SA, et al. Low- vs. High-Dose Neoadjuvant Radiation in Trimodality Treatment of Locally Advanced Esophageal Cancer. J Gastrointest Surg. 2019;23:885-94.

25. Semenkovich TR, Samson PP, Hudson JL, et al. Induction Radiation Therapy for Esophageal Cancer: Does Dose Affect Outcomes? Ann Thorac Surg. 2018;107:903-11.

26. Mandard A, Dalibard F, Mandard J, et al. Pathologic assessment of tumor regression after preoperative chemoradiotherapy of esophageal carcinoma. Clinicopathologic correlations. Cancer. 1994;73:2680-6.

27. Becker K, Mueller JD, Schulmacher C, et al. Histomorphology and grading of regression in gastric carcinoma treated with neoadjuvant chemotherapy. Cancer. 2003;98:1521-30.

28. Ajani JA, Correa AM, Walsh GL, et al. Trimodality therapy without a platinum compound for localized carcinoma of the esophagus and gastroesophageal junction. Cancer. 2010;116:1656-63.

29. Kushida T, Nohara S, Yoshino K, et al. Utility of weekly docetaxel combined with preoperative radiotherapy for locally advanced esophageal cancer from pathological analysis. Dis Esophagus. 2014;27:368-73.

30. Shapiro J, van Hagen P, Hester F. Prolonged Time to Surgery After Neoadjuvant Chemoradiotherapy Increases Histopathological Response Without Affecting Survival in Patients With Esophageal or Junctional Cancer. Ann Surg. 2014;260:807-14.

31. Meredith KL, Weber JM, Turaga KK, et al. Pathologic response after neoadjuvant therapy is the major determinant of survival in patients with esophageal cancer. Ann Surg Oncol. 2010;17:1159-67.

32. Lv HW, Li Y, Zhou MH, Cheng JW, Xing WQ. Remnant lymph node metastases after neoadjuvant therapy and surgery in patients with pathologic T0 esophageal carcinoma impact on prognosis: A systematic review and meta-analysis. Medicine (Baltimore). 2017;96:1-6.

33. Depypere L, Vervloet G, Lerut T, et al. ypT0N+: The unusual patient with pathological complete tumor response but with residual lymph node disease after neoadjuvant chemoradiation for esophageal cancer, what's up? J Thorac Dis. 2018;10:2771-8.

34. Schurink B, Seesing MFJ, Goense L, et al. ypT0N+ status in oesophageal cancer patients: Location of residual metastatic lymph nodes with regards to the neoadjuvant radiation field. Eur J Surg Oncol. 2018;45:454-9.

35. Hölscher AH, Drebber U, Schmidt H, Bollschweiler E. Prognostic classification of histopathologic response to neoadjuvant therapy in esophageal adenocarcinoma. Ann Surg. 2014;260:779-85.

36. Reynolds JV, Muldoon C, Hollywood D, et al. Long-term outcomes following neoadjuvant chemoradiotherapy for esophageal cancer. Ann Surg. 2007;245:707-16. 


\section{SUPPLEMENTARY MATERIAL}

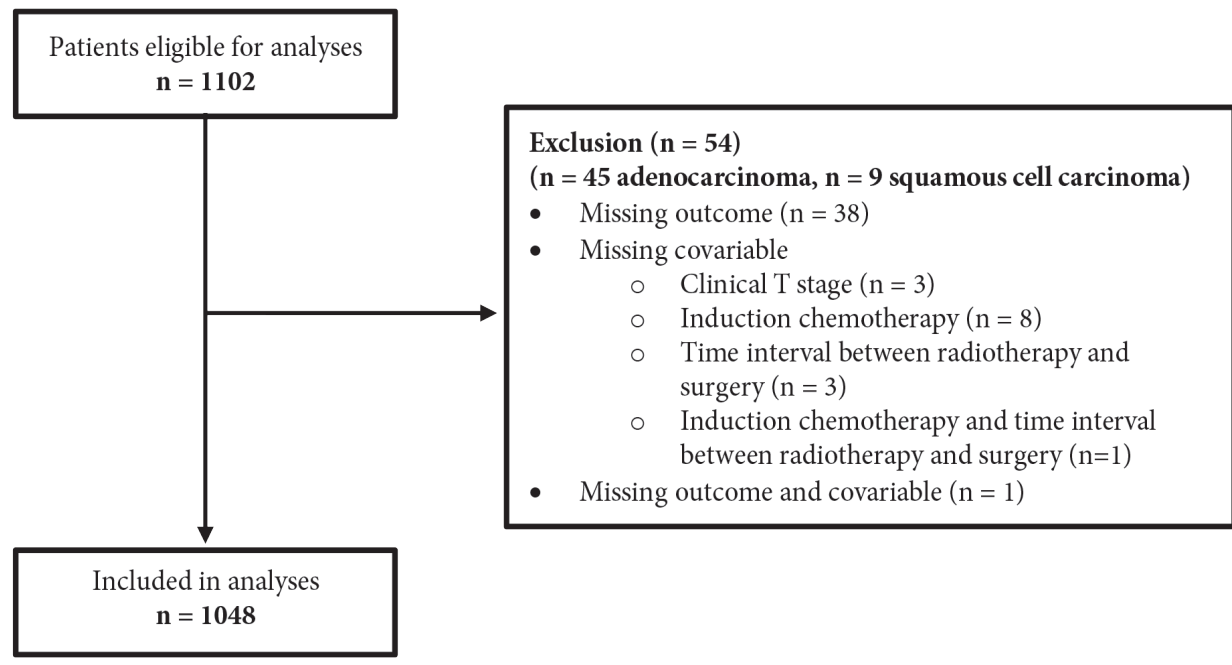

Supplementary figure 1 . Consort diagram of the study 


\section{A. Entire cohort}

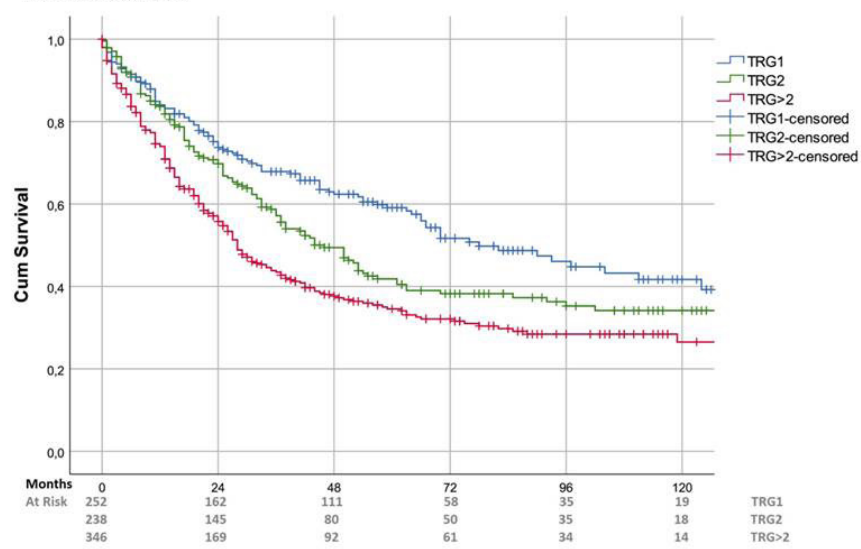

B. Adenocarcinoma

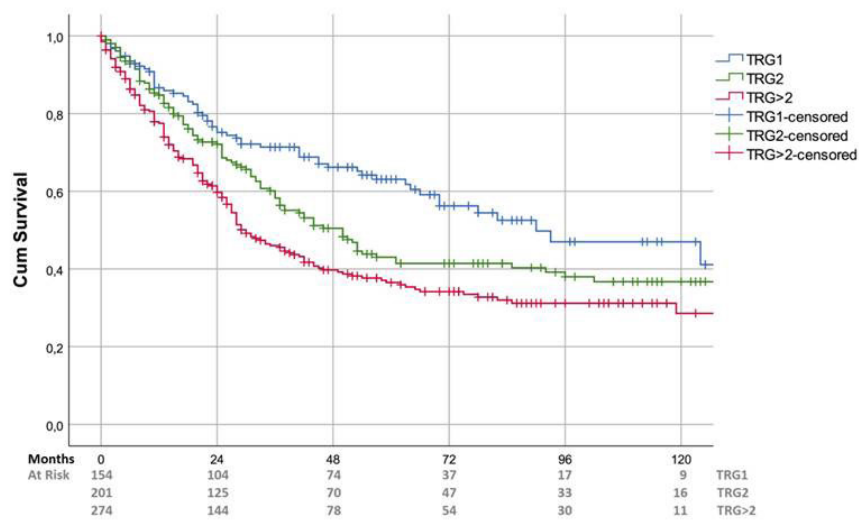

\section{Squamous cell carcinoma}

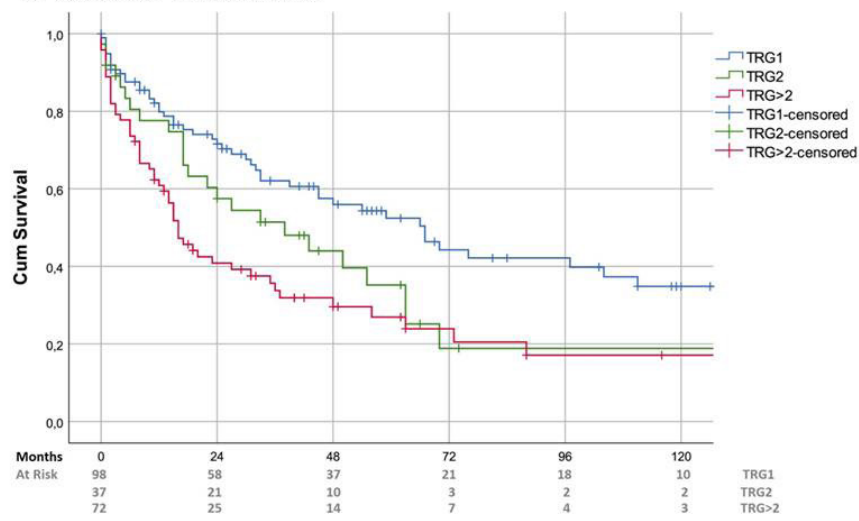

Supplementary figure 2. Overall survival curves of tumour regression grade (TRG) 1 (pathologic complete response; pCR, blue curve) vs. TRG 2 (green curve) vs. TRG >2 (red curve) in the entire patient cohort (A) and according to histologic subgroups (adenocarcinoma: B, squamous cell carcinoma: C). 

PART II

Treatment response assessment 


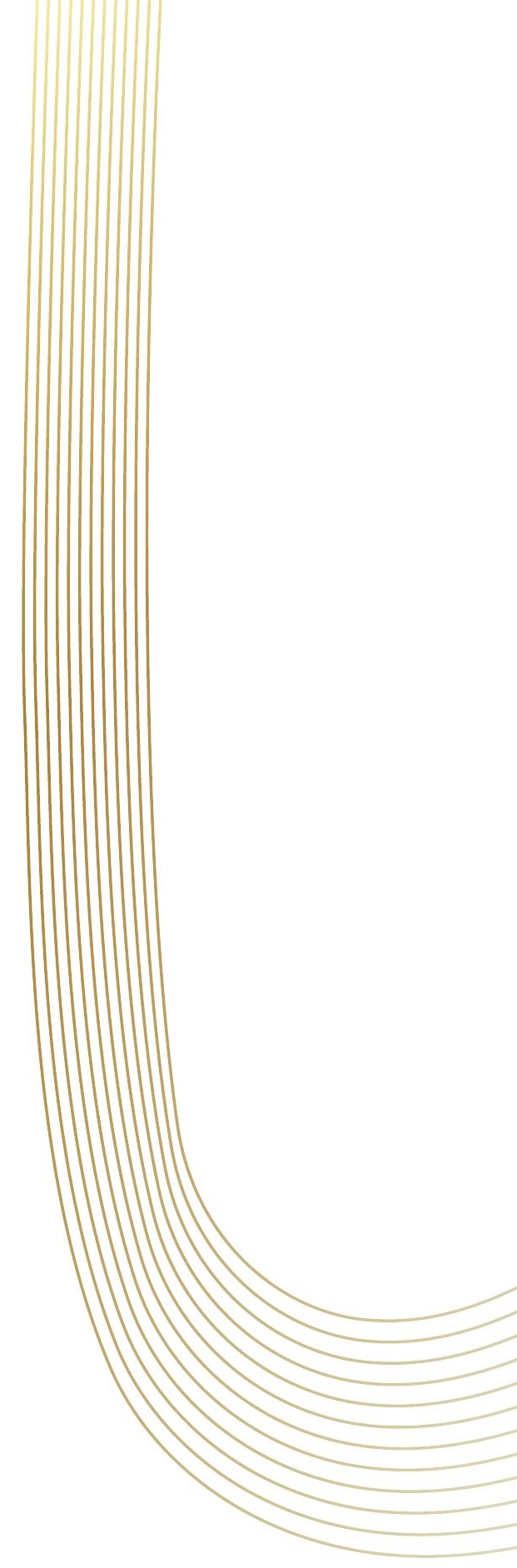


Chapter 5

Preoperative prediction of pathologic response to neoadjuvant chemoradiotherapy in patients with esophageal cancer using ${ }^{18} \mathrm{~F}-\mathrm{FDG}$ PET/CT and DW-MRI: a prospective multicenter study

International Journal of Radiation Oncology Biology Physics 2020

A.S. Borggreve*, L. Goense*, P.S.N. van Rossum, S.E. Heethuis, R. van Hillegersberg, J.J.W. Lagendijk, M.G.E.H. Lam, A.L.H.M.W. van Lier, S. Mook, J.P. Ruurda, M. van Vulpen, F.E.M. Voncken, B.M.P. Aleman, A. Bartels-Rutten, J. Ma, P. Fang, B.C. Musall, S.H. Lin, G.J. Meijer

${ }^{*}$ Joint first authorship. 


\section{Abstract}

\section{Background}

Accurate preoperative prediction of pathologic response to neoadjuvant chemoradiotherapy (nCRT) in esophageal cancer patients could enable to forgo esophagectomy in patients with a pathologic complete response ( $\mathrm{pCR}$ ). This study aimed to evaluate the individual and combined value of ${ }^{18} \mathrm{~F}$-fluorodeoxyglucose positron emission tomography with integrated computed tomography $\left({ }^{18} \mathrm{~F}-\mathrm{FDG}\right.$ PET/CT) and diffusion-weighted magnetic resonance imaging (DW-MRI) during and after nCRT to predict pathologic response in esophageal cancer patients.

\section{Methods}

In this multicenter prospective study, patients scheduled to receive nCRT followed by esophagectomy for esophageal cancer underwent ${ }^{18} \mathrm{~F}-\mathrm{FDG}$ PET/CT and DW-MRI scanning prior to start of nCRT, during nCRT, and before esophagectomy. Response to nCRT was based on histopathological evaluation of the resection specimen. Relative changes in ${ }^{18} \mathrm{~F}-\mathrm{FDG}$ PET/CT and DW-MRI parameters were compared between patients with pCR and non-pCR groups. Multivariable Ridge regression analyses with bootstrapped c-indices were performed to evaluate the individual and combined value of ${ }^{18} \mathrm{~F}-\mathrm{FDG}$ PET/CT and DW-MRI.

\section{Results}

pCR was found in $26.1 \%$ of 69 patients. Relative changes in ${ }^{18} \mathrm{~F}-\mathrm{FDG}$ PET/CT parameters after nCRT $\left(\triangle S U V_{\text {mean,post }} \mathrm{p}=0.016\right.$, and $\left.\Delta \mathrm{TLG}_{\text {post }} \mathrm{p}=0.024\right)$, as well as changes in DW-MRI parameters during $\mathrm{nCRT}\left(\triangle \mathrm{ADC}_{\text {during }} \mathrm{p}=0.008\right)$ were significantly different between $\mathrm{pCR}$ and non-pCR. A c-statistic of 0.84 was obtained for a model with $\triangle \mathrm{ADC}_{\text {during }}, \triangle \mathrm{SUV}_{\text {mean,post }}$ and histology in classifying patients as $\mathrm{pCR}$ (versus 0.82 for $\triangle \mathrm{ADC}_{\text {during }}$ and 0.79 for $\Delta \mathrm{SUV}_{\text {mean,post }}$ alone).

\section{Conclusions}

Changes on ${ }^{18} \mathrm{~F}-\mathrm{FDG}$ PET/CT after nCRT and early changes on DW-MRI during nCRT can help identify pCR to nCRT in esophageal cancer. Moreover, ${ }^{18} \mathrm{~F}-\mathrm{FDG}$ PET/CT and DW-MRI might be of complementary value in the assessment of pCR. 


\section{Introduction}

Neoadjuvant chemoradiotherapy (nCRT) followed by surgery is the preferred treatment with curative intent for patients with locally advanced esophageal cancer. ${ }^{1}$ Through tumor downsizing and downstaging, nCRT improves locoregional control and overall survival rates compared to surgery alone. ${ }^{2-4}$ Many studies have reported that the degree of tumor regression in response to $\mathrm{nCRT}$ is directly related to long-term survival, with pathologic complete responders having the most favorable long-term prognosis. ${ }^{3,5}$ The absence of viable tumor cells at the site of the primary tumor in pathologic complete responders raises the question whether a surgical resection is of benefit for patients who might have already been cured locoregionally by nCRT alone. ${ }^{6}$ Accurate prediction of pathologic complete response ( $\mathrm{pCR}$ ) before surgery could potentially allow patients to forgo surgery and would enable researchers to study the feasibility and outcome of an organ-preserving strategy after chemoradiotherapy. Conversely, patients with a poor response to nCRT are likely to benefit less or not at all from nCRT, but are exposed to its side-effects. A reliable identification of poor responders during nCRT may thus be also beneficial, as ineffective therapy could be stopped and/or alternative treatment strategies (e.g. additional neoadjuvant treatment or upfront surgery) could be explored.

Unfortunately, most studied modalities - including endoscopic biopsy, endoscopic ultrasonography (EUS) and computed tomography (CT) - yield unsatisfactory results for the evaluation of tumor response to nCRT. ${ }^{7-11}$ Metabolic and functional imaging modalities such as ${ }^{18} \mathrm{~F}$-fluorodeoxyglucose positron emission tomography with integrated computed tomography $\left({ }^{18} \mathrm{~F}-\mathrm{FDG}\right.$ PET/CT) and diffusion-weighted magnetic resonance imaging (DWMRI) may be more promising because they allow biological and microstructural characterization of tumors and visualization of treatment-induced changes before volumetric changes become apparent. ${ }^{9,12-18}$ However, the discriminatory ability of ${ }^{18} \mathrm{~F}-\mathrm{FDG}$ PET/CT alone has previously shown in a multitude of studies to be insufficient to guide clinical decision-making. ${ }^{14,19,20}$ The performance of DW-MRI and the quantitative apparent diffusion coefficient (ADC) are promising in predicting response to nCRT although they have been demonstrated only in two small single-center pilot studies. ${ }^{16,17}$

A multimodality imaging approach, in comparison to that of a single-modality, may provide complementary value for predicting pathologic response, with the ultimate goal of optimally guiding individualized treatment decision-making. Therefore, the aim of this prospective multicenter study was to evaluate the individual and combined value of ${ }^{18} \mathrm{~F}$-FDG PET/CT and DW-MRI during and after nCRT to predict pathologic response in patients who undergo nCRT for esophageal cancer, as well as to validate these findings for the prediction of survival. 


\section{Methods}

Three high volume institutions participated in this multicenter prospective study including the University Medical Center Utrecht (UMC Utrecht), the Netherlands Cancer Institute (NKI-AVL) and the University of Texas MD Anderson Cancer Center (MDACC). The study has been approved by the institutional review board of each institution separately, and all patients provided their written informed consent. The study was registered with ClinicalTrials.gov, number NCT02125448.

\section{Study population}

Patients presenting at any of the 3 participating institutions from October 2013 to July 2017 with newly diagnosed biopsy-proven esophageal cancer who were scheduled to receive nCRT followed by surgery were eligible for inclusion. Exclusion criteria are outlined in the Supplementary Methods. The results of first 20 of the 26 included patients from the MDACC have been published previously. ${ }^{17}$

\section{Treatment protocol}

At the UMC Utrecht and NKI-AVL, the neoadjuvant treatment regimen consisted of carboplatin/paclitaxel with concurrent radiotherapy (41.4 Gy in 23 fractions of 1.8 Gy). ${ }^{4}$ At the MDACC, the regimen consisted of 5-fluorouracil with either platinum or taxanebased chemotherapy and concurrent radiotherapy (50.4 Gy in 28 fractions of $1.8 \mathrm{~Gy}$ ). All patients were treated with an intensity-modulated radiation therapy (IMRT) technique. At a median of 8 weeks (interquartile range [IQR]: 7-10 weeks) after completion of nCRT, patients underwent a transhiatal or transthoracic esophagectomy with two-field lymphadenectomy and gastric conduit reconstruction with either cervical or intrathoracic anastomosis, depending on patient characteristics and local preference.

\section{Histopathological assessment}

Histopathological tumor regression of the surgical resection specimen was assessed by specialized gastrointestinal pathologists who were blinded for the results of the ${ }^{18} \mathrm{~F}-\mathrm{FDG}$ PET/CT and DW-MRI scans. Patients were staged in accordance with the 7 th edition of the Union for International Cancer Control (UICC). ${ }^{21}$ The following tumor regression groups were scored: absence of residual cancer cells (TRG 1), 1-10\% residual cancer cells (TRG 2), 10-50\% residual cancer cells (TRG 3) and $>50 \%$ residual cancer cells (TRG 4).22

\section{Image acquisition}

Patients underwent ${ }^{18} \mathrm{~F}-\mathrm{FDG}$ PET/CT scanning at 3 time points: within a median of 3 weeks (IQR: 2 - 4 weeks) before nCRT, during nCRT at a median of 13 days (IQR: 10 - 15 days) 
after initiation of nCRT, and 5 weeks (IQR: 4.5 - 7 weeks) after completion of nCRT and before surgery (median: 14 days [IQR: 9 - 32 days] between ${ }^{18}$ F-FDG PET/CT scan and surgery).

Patients underwent DW-MRI scanning using 3 different b-values $(b=0,200$ and $800 \mathrm{~s} /$ $\mathrm{mm}^{2}$ ) at the same 3 time points as the ${ }^{18} \mathrm{~F}-\mathrm{FDG}$ PET/CT scans. Only the baseline DW-MRI scan was generally not performed on the exact same day as the baseline ${ }^{18} \mathrm{~F}-\mathrm{FDG}$ PET/CT scan, since the latter was part of the diagnostic work-up and the DW-MRI scan was acquired after patients were included in the study. The baseline DW-MRI scan was in all cases acquired within 2 weeks (median: 5 days, IQR: $1-6$ days) before nCRT. Detailed image acquisition parameters are presented in the Supplementary Methods.

\section{Image analysis}

Details on the ${ }^{18} \mathrm{~F}-\mathrm{FDG}$ PET/CT and DW-MRI tumor delineation methodology are included in the Supplementary Methods. At each of the 3 time points, the following 4 quantitative metrics were extracted from the ${ }^{18} \mathrm{~F}-\mathrm{FDG}$ PET/CT primary tumor delineations: mean and maximum standardized uptake value $\left(\mathrm{SUV}_{\text {mean }}, \mathrm{SUV}_{\text {max }}\right)$, metabolic tumor volume (MTV), and total lesion glycolysis (TLG) calculated as the product of MTV and $\mathrm{SUV}_{\text {mean }}{ }^{23}$ From the DW-MRI primary tumor delineations, the mean ADC values were extracted from the ADC map. The relative changes in percent $(\Delta \%)$ of these ${ }^{18} \mathrm{~F}-\mathrm{FDG}$ PET/CT and DW-MRI parameters between baseline scans and the scans during and after nCRT were calculated and considered as possible predictors in the analysis based on prior knowledge (i.e. $\triangle \mathrm{ADC}$, $\Delta \mathrm{SUV}_{\text {mean }}, \Delta \mathrm{SUV}_{\max }$ and $\left.\Delta \mathrm{TLG}\right) .^{16,17,20,24-27}$

\section{Statistical analysis}

The relative changes of the ${ }^{18} \mathrm{~F}-\mathrm{FDG}$ PET/CT and DW-MRI parameters were compared between patients with pCR (TRG 1) and non-pCR (TRG 2-4), and between good responders (TRG 1-2, GR) and poor responders (TRG 3-4, non-GR) to validate findings of previous pilot-studies using the Mann-Whitney $U$ test with Benjamin-Hochberg corrections. ${ }^{16,17,20,24-26,28,29}$ The ability of single modality ${ }^{18}$ F-FDG PET/CT and DW-MRI parameters to discriminate between different pathologic response groups was quantified using the area under the receiver operating characteristic curve (c-statistic). The complementary value of ${ }^{18} \mathrm{~F}$-FDG PET/CT and DW-MRI parameters, in addition to histopathological tumor type which known to impact pathologic response to nCRT, was assessed using multivariable penalized Ridge regression models to reduce model overfitting in a situation with few events per variable. ${ }^{30}$ The best model for $\mathrm{pCR}$ prediction was validated for the prediction of overall survival (OS) and disease free survival (DFS) using multivariable Cox regression analysis. Detailed statistical methods are presented in the Supplementary Methods. 
Relative changes during treatment
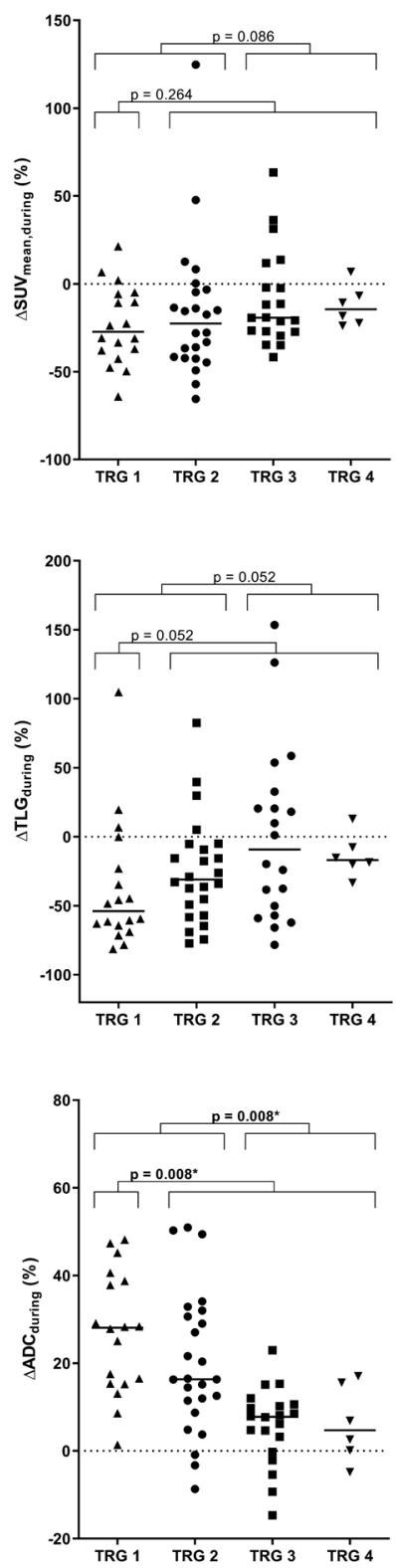

Relative changes after treatment
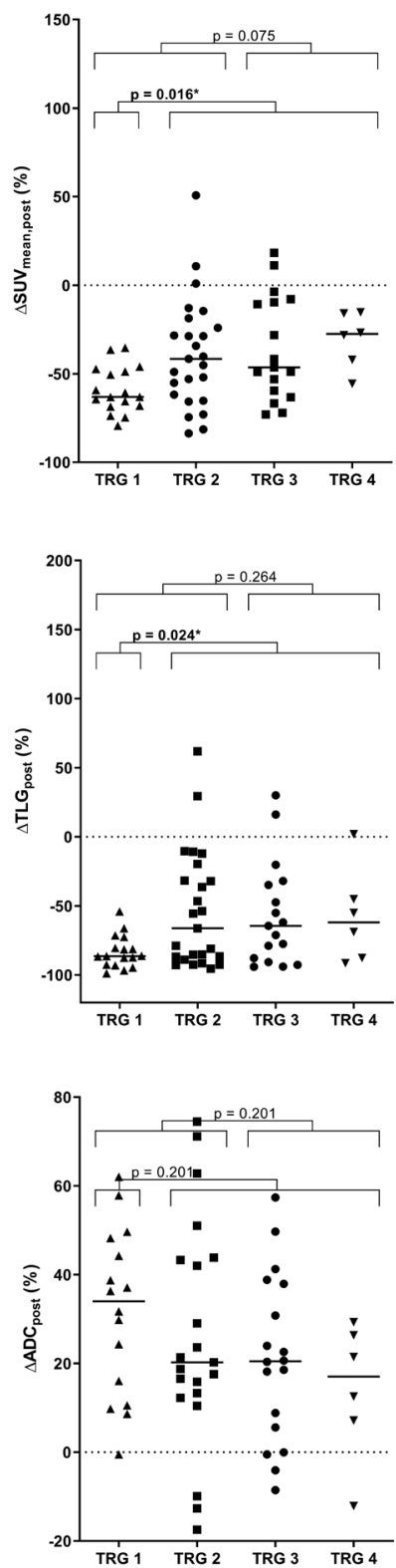

Figure 1. Relative changes in ${ }^{18} \mathrm{~F}-\mathrm{FDG}$ PET/CT and DW-MRI parameters during and after neoadjuvant chemoradiotherapy in all patients, categorized by histopathologic tumor regression grades (TRG). The solid horizontal lines represent group medians.

Significant differences between the groups based on the Mann-Whitney U test followed by Benjamini-Hochberg adjustment are marked with an asterisk $\left(^{*}\right)$. 


\section{Results}

\section{Study population}

Between October 2013 and July 2017, a total of 82 consecutive patients with newly diagnosed esophageal cancer who underwent standard diagnostic work-up signed informed consent. A total of 13 patients were excluded from the analysis (withdrawal from study participation $[n=4]$, unexpected distant metastatic disease $[n=3]$, no tumor signal on the baseline ${ }^{18} \mathrm{~F}-\mathrm{FDG}$ PET/CT or DW-MRI [ $n=3]$, small tumor volume $[<2 \mathrm{ml}, \mathrm{n}=2]$ and refusal of surgery $[n=1])$. The remaining 69 patients, with a total of $203{ }^{18} \mathrm{~F}-\mathrm{FDG}$ PET/CT scans and 199 DW-MRI scans, were eligible for analysis (Supplementary Figure 1).

Patients had a mean age of 61.0 years ( $S D \pm 9.2$ years), and $88.4 \%(n=61)$ were male. Histologic tumor types included adenocarcinoma $(n=57,82.6 \%)$, squamous cell carcinoma $(\mathrm{n}=11,15.9 \%)$ or undifferentiated large cell carcinoma type $(\mathrm{n}=1,1.4 \%)$. Histopathological assessment of the surgical resection specimen demonstrated pCR (i.e. TRG 1) in 18 patients (26.1\%), and GR (i.e. TRG 1-2) in 43 patients (62.3\%) after nCRT (see also Supplementary Table 1). Patients with a squamous cell carcinoma had a significantly higher probability of pCR and GR compared to patients with adenocarcinoma (Table 1). No significant differences were observed in clinical T stages, nCRT regimens or time intervals from nCRT to surgery between patients with pCR and non-pCR, or between patients with GR and non-GR (Table 1). Detailed patient and tumor characteristics are outlined in Table 1.

\section{Relative changes in ${ }^{18} \mathrm{~F}$-FDG PET/CT and DW-MRI parameters}

The relative change in tumor $\mathrm{SUV}_{\text {mean, }}$ SUV $_{\text {max }}$ TLG and $\mathrm{ADC}_{\text {mean }}$ during and after nCRT for pCR and GR patients are presented in Table 2 and illustrated in Figure 1. In Figure 2 ${ }^{18} \mathrm{~F}-\mathrm{FDG}$ PET/CT and MRI scans of a patient with pCR are presented.

The changes in $\mathrm{SUV}_{\text {mean, }} \mathrm{SUV}_{\text {max }}$ and TLG from baseline ${ }^{18} \mathrm{~F}-\mathrm{FDG}$ PET/CT scans to scans acquired during nCRT were not significantly different between patients with pCR versus non-pCR (Table 2). Changes in SUV $\mathrm{Sean}_{\text {man }}$ and from baseline ${ }^{18} \mathrm{~F}-\mathrm{FDG}$ PET/CT scans to scans acquired after completion of nCRT, however, were significantly different between pCR and non-pCR $\left(\triangle \mathrm{SUV}_{\text {mean,post }}\right.$ [median, IQR]: -63\% [-68\%, -49\%] for pCR versus $-42 \%$ $[-58 \%,-16 \%]$ for non-pCR, $\mathrm{p}=0.016$, and $\Delta \mathrm{TLG}_{\text {post }}$ [median, IQR]: $-86 \%[-93 \%,-81 \%]$ for $\mathrm{pCR}$ versus $-65 \%[-88 \%,-32 \%]$ for non-pCR, $\mathrm{p}=0.024)$. Regarding $\mathrm{GR}$, none of the changes in ${ }^{18} \mathrm{~F}$-FDG PET/CT parameters from baseline to either during or after completion of nCRT were significantly different between GR and non-GR (Table 2).

The relative increase in tumor ADC from baseline DW-MRI scans to scans acquired during $\mathrm{nCRT}\left(\triangle \mathrm{ADC}_{\text {during }}\right)$ was significantly associated with pCR (median, IQR: 28\% [15\%, 39\%] for $\mathrm{pCR}$ versus $11 \%[4 \%, 17 \%]$ for non-pCR, $\mathrm{p}=0.008)$. In contrast, relative changes in ADC from baseline to scans after completion of nCRT $\left(\triangle \mathrm{ADC}_{\text {post }}\right)$ were not significantly 
Table 1. Clinical characteristics of the study population and univariable analyses between predetermined clinical predictors and response groups.

\begin{tabular}{|c|c|c|c|c|c|c|c|}
\hline Characteristics & $\begin{array}{c}\text { All } \\
\text { n (\%) }\end{array}$ & $\begin{array}{c}\text { pCR } \\
(\mathbf{n}=18) \\
n(\%)\end{array}$ & $\begin{array}{c}\text { non-pCR } \\
(\mathbf{n}=5 \mathbf{1}) \\
\text { n }(\%)\end{array}$ & $\begin{array}{c}\mathrm{p}- \\
\text { value }\end{array}$ & $\begin{array}{c}\text { GR } \\
(n=43) \\
n(\%)\end{array}$ & $\begin{array}{c}\text { non-GR } \\
(\mathbf{n}=26) \\
\text { n }(\%)\end{array}$ & $\begin{array}{c}\text { p- } \\
\text { value }\end{array}$ \\
\hline $\begin{array}{l}\text { Age at diagnosis, in years } \\
(\text { mean } \pm S D)\end{array}$ & $61.0 \pm 9.2$ & $60.1 \pm 8.8$ & $61.4 \pm 9.4$ & NA & $61.1 \pm 9.4$ & $60.9 \pm 9.0$ & NA \\
\hline Sex & & & & NA & & & NA \\
\hline Male & $61(88)$ & $3(17)$ & $5(10)$ & & $5(12)$ & $3(12)$ & \\
\hline Female & $8(12)$ & $15(83)$ & $46(90)$ & & $38(88)$ & $23(88)$ & \\
\hline $\mathrm{BMI}$, in $\mathbf{k g} / \mathbf{m}^{2}($ mean $\pm \mathrm{SD})$ & $25.7 \pm 3.8$ & $24.7 \pm 5.2$ & $26.1 \pm 3.1$ & $\mathrm{NA}$ & $25.2 \pm 4.2$ & $26.7 \pm 2.8$ & NA \\
\hline $\begin{array}{l}\text { WHO performance status at } \\
\text { diagnosis }\end{array}$ & & & & NA & & & NA \\
\hline 0 & $27(39)$ & $5(28)$ & $22(43)$ & & $18(42)$ & $9(35)$ & \\
\hline 1 & $40(58)$ & $12(67)$ & $28(55)$ & & $24(56)$ & $16(62)$ & \\
\hline 2 & $2(3)$ & $1(6)$ & $1(2)$ & & $1(2)$ & $1(4)$ & \\
\hline Tumor location & & & & NA & & & NA \\
\hline Proximal esophagus & $1(1)$ & $0(0)$ & $1(2)$ & & $1(2)$ & $0(0)$ & \\
\hline Middle esophagus & $9(13)$ & $5(28)$ & $4(8)$ & & $8(19)$ & $1(4)$ & \\
\hline Distal esophagus & $40(58)$ & $6(33)$ & $34(67)$ & & $22(51)$ & $18(69)$ & \\
\hline Gastroesophageal junction & $19(28)$ & $7(39)$ & $12(24)$ & & $12(28)$ & $7(27)$ & \\
\hline Clinical T status ${ }^{*}$ & & & & 0.233 & & & 0.698 \\
\hline $\mathrm{T} 2$ & $9(13)$ & $2(11)$ & $7(14)$ & & $6(14)$ & $3(12)$ & \\
\hline T3 & $59(86)$ & $15(83)$ & $44(86)$ & & $36(84)$ & $23(88)$ & \\
\hline $\mathrm{T} 4$ & $1(1)$ & $1(6)$ & $0(0)$ & & $1(2)$ & $0(0)$ & \\
\hline Clinical N status ${ }^{*}$ & & & & NA & & & NA \\
\hline N0 & $23(33)$ & $6(33)$ & $17(33)$ & & $15(35)$ & $8(31)$ & \\
\hline N1 & $26(38)$ & $5(28)$ & $21(41)$ & & $12(28)$ & $14(54)$ & \\
\hline $\mathrm{N} 2$ & $18(26)$ & $7(39)$ & $11(22)$ & & $15(35)$ & $3(12)$ & \\
\hline N3 & $2(3)$ & $0(0)$ & $2(4)$ & & $1(2)$ & $1(4)$ & \\
\hline Histology & & & & 0.001 & & & 0.069 \\
\hline Adenocarcinoma & $57(83)$ & $10(56)$ & $47(92)$ & & $32(74)$ & $25(96)$ & \\
\hline Squamous cell carcinoma & $11(16)$ & $7(39)$ & $4(8)$ & & $10(23)$ & $1(4)$ & \\
\hline $\begin{array}{l}\text { Undifferentiated large cell } \\
\text { carcinoma }\end{array}$ & $1(1)$ & $1(6)$ & $0(0)$ & & $1(2)$ & $0(0)$ & \\
\hline Tumor regression grade & & & & NA & & & NA \\
\hline TRG 1 (pCR) & $18(26)$ & $18(100)$ & NA & & $18(42)$ & NA & \\
\hline TRG 2 & $25(36)$ & NA & $25(49)$ & & $25(58)$ & NA & \\
\hline TRG 3 & $20(29)$ & NA & $20(39)$ & & NA & $20(77)$ & \\
\hline TRG 4 & $6(9)$ & NA & $6(12)$ & & NA & $6(23)$ & \\
\hline $\begin{array}{l}\text { Neoadjuvant chemoradiotherapy } \\
\text { regimen }\end{array}$ & & & & 0.902 & & & 0.357 \\
\hline Carboplatin/paclitaxel $+41.4 \mathrm{~Gy}$ & $43(45)$ & $11(61)$ & $32(63)$ & & $25(58)$ & $18(69)$ & \\
\hline 5-fluorouracil-based + 50.4 Gy & $26(38)$ & $7(39)$ & $19(37)$ & & $18(42)$ & $8(31)$ & \\
\hline $\begin{array}{l}\text { Time interval nCRT to surgery } \\
\text { (median, IQR) }\end{array}$ & $8(7-10)$ & $8(7-9)$ & $8(7-10)$ & 0.269 & $8(7-10)$ & $8(6-9)$ & 0.630 \\
\hline
\end{tabular}

$B M I$ body mass index at diagnosis; IQR interquartile range; $N A$ not applicable; $p C R$ pathologic complete response; TRG tumor regression grade; WHO World Health Organization

* Clinical $\mathrm{T}$ and $\mathrm{N}$ status are based on AJCC TNM 7th edition.

Note. P-values in this table were based on $\chi^{2}$ or Fisher's exact test for categorical variables and the Mann-

Whitney $\mathrm{U}$ test for continuous variables. 
different between pCR and non-pCR groups. These findings were consistent with the association between changes in $\mathrm{ADC}$ and $\mathrm{GR}$ versus non-GR $\left(\triangle \mathrm{ADC}_{\text {during }}\right.$ [median, IQR]: $20 \%[13 \%, 33 \%]$ for GR versus $8 \%[0 \%, 11 \%]$ for non-GR, $\mathrm{p}=0.008$, and $\triangle \mathrm{ADC}_{\text {post }}$ [median, IQR]: $24 \%$ [13\%, 44\%] for GR versus $21 \%$ [6\%, 30\%] for non-GR, $\mathrm{p}=0.201$, Table 2).

\section{Complementary value ${ }^{18} \mathrm{~F}$-FDG PET/CT and DW-MRI parameters}

To evaluate the complementary value of ${ }^{18} \mathrm{~F}$-FDG PET/CT and DW-MRI parameters for $\mathrm{pCR}$ prediction, receiver operating characteristic (ROC) analyses for $\triangle \mathrm{ADC}_{\text {during }}$ combined with $\triangle \mathrm{SUV}_{\text {mean,post }}$ and histology showed a superior bootstrapped c-statistic in comparison with their individual values and histology $(0.83 \text { [95\% CI: } 0.74-0.94]_{\text {for }} \Delta \mathrm{ADC}_{\text {during }}$ and $\Delta \mathrm{SUV}_{\text {mean,post }}$, versus 0.81 [95\% CI: 0.70 - 0.93] for $\triangle \mathrm{ADC}_{\text {during }}$ and 0.79 [95\% CI: 0.67 - 0.90] for $\triangle \mathrm{SUV}_{\text {mean,post }}$, all models with histology, Table 3 and Figure $\left.3 \mathrm{a}\right)$. Furthermore, the model with $\triangle \mathrm{ADC}_{\text {during }} \Delta \mathrm{SUV}_{\text {mean,post }}$ and histology also demonstrated the best global model fit in terms of the lowest AIC (Supplementary Table 2). ROC curve analyses of ${ }^{18} \mathrm{~F}-\mathrm{FDG}$ PET/ $\mathrm{CT}$ and DW-MRI parameters acquired during treatment for prediction of GR did not demonstrate complementary value of $\Delta \mathrm{ADC}_{\text {during }}$ and $\Delta \mathrm{SUV}_{\text {max,during, }}$ but demonstrated a
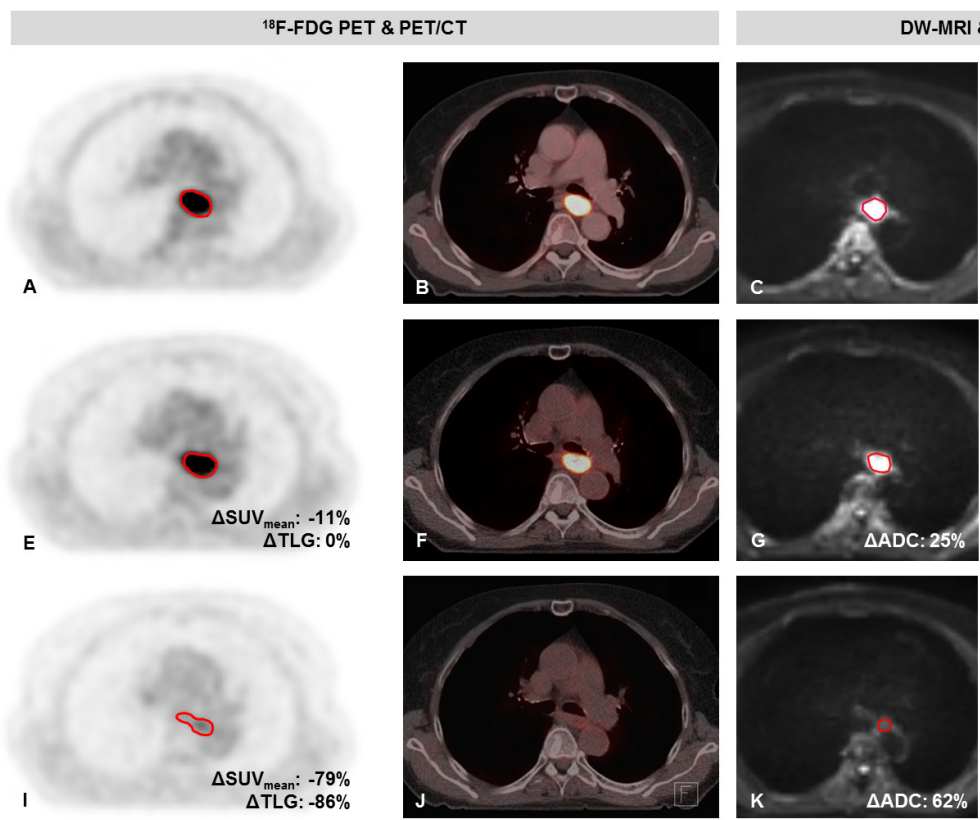

DW-MRI \& T2W MRI
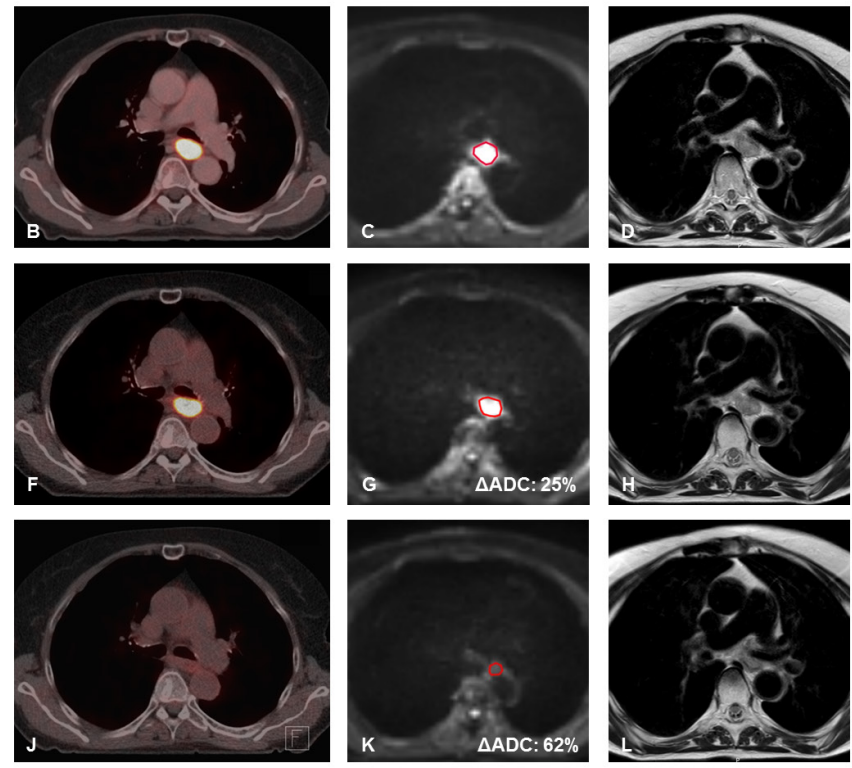

Figure 2. Patient with a cT3N2M0 mid-esophageal squamous cell carcinoma with a pathologic complete response to neoadjuvant chemoradiotherapy (TRG 1). ${ }^{18} \mathrm{~F}-\mathrm{FDG}$ PET images (A, E, I), fused PET/CT images (B, F, J), diffusion-weighted images $\left(\mathrm{b}\right.$-value $\left.=200 \mathrm{~s} / \mathrm{mm}^{2}\right)(\mathrm{C}, \mathrm{G}, \mathrm{K})$ and T2 weighted images $(\mathbf{D}, \mathbf{H}, \mathbf{L})$ on a $1.5 \mathrm{~T}$ MR scanner before neoadjuvant chemoradiotherapy (nCRT) (A-D), during nCRT (E-H), and after nCRT (I-L). 
high bootstrapped c-statistic for $\triangle \mathrm{ADC}_{\text {during }}$ with histology $(0.82$ [95\% CI: $0.75-0.94$ ] for $\Delta \mathrm{ADC}_{\text {during }}$ and $\Delta \mathrm{SUV}_{\text {max,during, }}$, versus 0.83 [95\% CI: $\left.0.73-0.92\right]_{\text {for }} \Delta \mathrm{ADC}_{\text {during }}$ and 0.74 [95\% CI: $0.60-0.88$ ] for $\Delta \mathrm{SUV}_{\text {max,during, }}$, all models with histology, Table 3 and Figure $3 \mathrm{~b}$ ). The model with $\triangle \mathrm{SUV}_{\text {max,during }}$ and histology demonstrated the best global model fit in terms lowest AIC (Supplementary Table 2).

Model calibration plots of all 8 models as described in Table 3 are depicted in Supplementary Figure 2, and show variable calibration upon visual inspection. In general, the best model for pCR in terms of highest c-statistic and lowest AIC (the model with DW-MRI, PET-CT and histology, i.e. model 4 in Table 3) was slightly overoptimistic and tended to underestimate the observed probability of pCR, whereas the best model for GR in terms of highest c-statistic (the model with DW-MRI and histology, i.e. model 2 in Table 3) was better calibrated.

Table 2. Relative changes in ${ }^{18} \mathrm{~F}-\mathrm{FDG}$ PET/CT and DW-MRI parameters between pathologic complete response (TRG 1) and non-pathologic complete response (TRG 2-4), as well as between good response (TRG 1-2) and poor response (TRG 3-4).

\begin{tabular}{|c|c|c|c|c|c|c|}
\hline & & \multicolumn{2}{|c|}{ Median (IQR) } & \multirow{2}{*}{\multicolumn{2}{|c|}{$\begin{array}{cc} & \begin{array}{c}\text { Benjamini- } \\
\text { Hochberg } \\
\text { p- }\end{array} \\
\text { adjusted } \\
\text { value }^{*} & \text { p-value } \\
\end{array}$}} & \multirow[b]{2}{*}{$\begin{array}{c}\text { c- } \\
\text { statistic }\end{array}$} \\
\hline & & $\begin{array}{c}\mathrm{pCR} \\
(\mathrm{TRG} 1) \\
(\mathrm{n}=18)\end{array}$ & $\begin{array}{c}\text { non-pCR } \\
(\text { TRG 2-4) } \\
(n=51)\end{array}$ & & & \\
\hline \multirow{4}{*}{$\begin{array}{l}\text { During } \\
\text { treatment }\end{array}$} & $\Delta \mathrm{SUV}_{\text {mean }}(\%)$ & $-27(-38,-6)$ & $-19(-33,-3)$ & 0.246 & 0.264 & 0.59 \\
\hline & $\Delta \mathrm{SUV}_{\max }(\%)$ & $-29(-46,-8)$ & $-23(-35,-2)$ & 0.408 & 0.408 & 0.57 \\
\hline & $\Delta \mathrm{TLG}_{\text {during }}(\%)$ & $-54(-64,-23)$ & $-20(-50,10)$ & 0.020 & 0.052 & 0.69 \\
\hline & $\Delta \mathrm{ADC}_{\text {during }}(\%)$ & $28(15,39)$ & $11(4,17)$ & 0.001 & 0.008 & 0.77 \\
\hline \multirow[t]{6}{*}{ Post treatment } & $\Delta \mathrm{SUV}_{\text {mean }}(\%)$ & $-63(-68,-49)$ & $-42(-58,-16)$ & 0.003 & 0.016 & 0.74 \\
\hline & $\Delta \mathrm{SUV}_{\max }(\%)$ & $-68(-78,-58)$ & $-54(-71,-31)$ & 0.026 & 0.052 & 0.68 \\
\hline & $\Delta \mathrm{TLG}_{\text {post }}(\%)$ & $-86(-93,-81)$ & $-65(-88,-32)$ & 0.006 & 0.024 & 0.72 \\
\hline & $\Delta \mathrm{ADC}_{\text {post }}(\%)$ & $34(13,46)$ & $20(10,38)$ & 0.139 & 0.201 & 0.63 \\
\hline & & \multicolumn{2}{|c|}{ Median (IQR) } & \multirow{2}{*}{\multicolumn{2}{|c|}{ 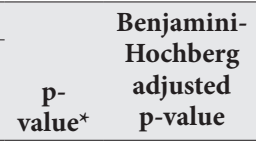 }} & \\
\hline & & $\begin{array}{c}\text { GR } \\
(\text { TRG 1-2) } \\
(n=43)\end{array}$ & $\begin{array}{c}\text { non-GR } \\
(\text { TRG 3-4) } \\
(n=26)\end{array}$ & & & $\begin{array}{c}c- \\
\text { statistic }\end{array}$ \\
\hline \multirow{4}{*}{$\begin{array}{l}\text { During } \\
\text { treatment }\end{array}$} & $\Delta \mathrm{SUV}_{\text {mean }}(\%)$ & $-26(-42,-5)$ & $-19(-27,-2)$ & 0.054 & 0.086 & 0.64 \\
\hline & $\Delta \mathrm{SUV}_{\max }(\%)$ & $-31(-44,-14)$ & $-12(-29,2)$ & 0.016 & 0.051 & 0.67 \\
\hline & $\Delta \mathrm{TLG}_{\text {during }}(\%)$ & $-37(-62,-9)$ & $-17(-38,20)$ & 0.024 & 0.052 & 0.66 \\
\hline & $\Delta \mathrm{ADC}_{\text {during }}(\%)$ & $20(13,33)$ & $7.3(0,11)$ & $<0.001$ & 0.008 & 0.84 \\
\hline \multirow[t]{4}{*}{ Post treatment } & $\Delta \mathrm{SUV}_{\text {mean }}(\%)$ & $-51(-66,-34)$ & $-42(-56,-11)$ & 0.042 & 0.075 & 0.65 \\
\hline & $\Delta \mathrm{SUV}_{\max }(\%)$ & $-63(-76,-44)$ & $-53(-74,-26)$ & 0.187 & 0.230 & 0.60 \\
\hline & $\Delta \mathrm{TLG}_{\text {post }}(\%)$ & $-81(-89,-54)$ & $-64(-88,-35)$ & 0.248 & 0.264 & 0.59 \\
\hline & $\Delta \mathrm{ADC}_{\text {post }}(\%)$ & $24(13,44)$ & $21(6,30)$ & 0.151 & 0.201 & 0.61 \\
\hline
\end{tabular}

$A D C$ apparent diffusion coefficient; $c$-statistic concordance statistic; GR good response; IQR interquartile range; $p C R$ pathologic complete response; $S D$ standard deviation; $S U V$ standardized uptake value; TLG tumor lesion glycolysis; TRG tumor regression grade

* p-value calculated based on Mann-Whitney U test 
ROC Curve for the probability of pCR as related to DW-MRI and ${ }^{18}$ F-FDG PET-CT parameters

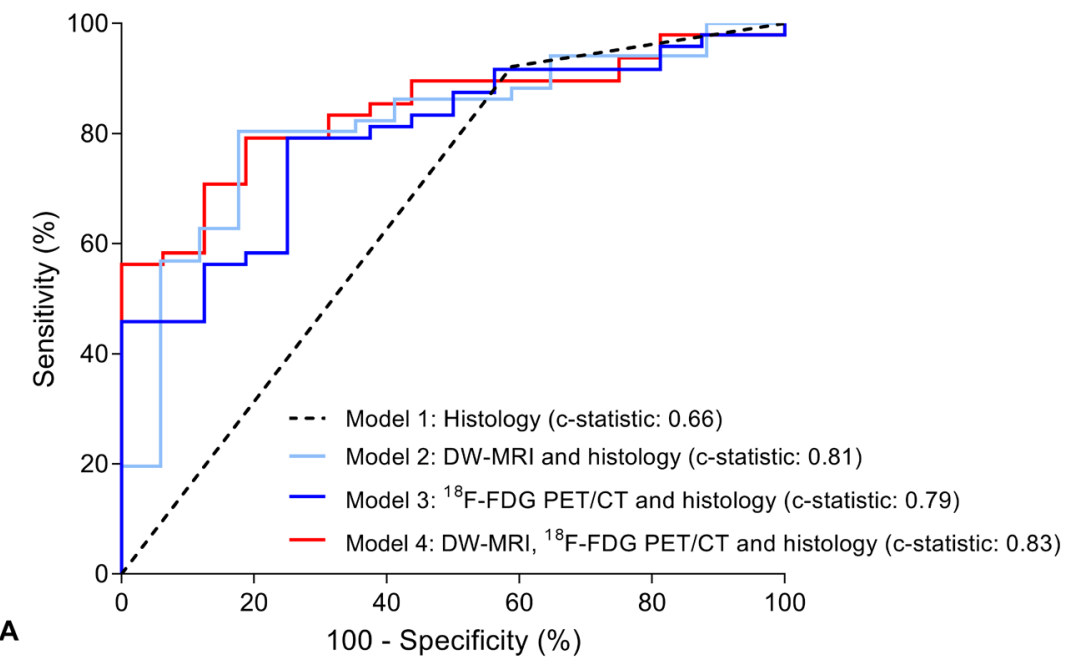

ROC Curve for the probability of GR as related to DW-MRI and ${ }^{18}$ F-FDG PET-CT parameters

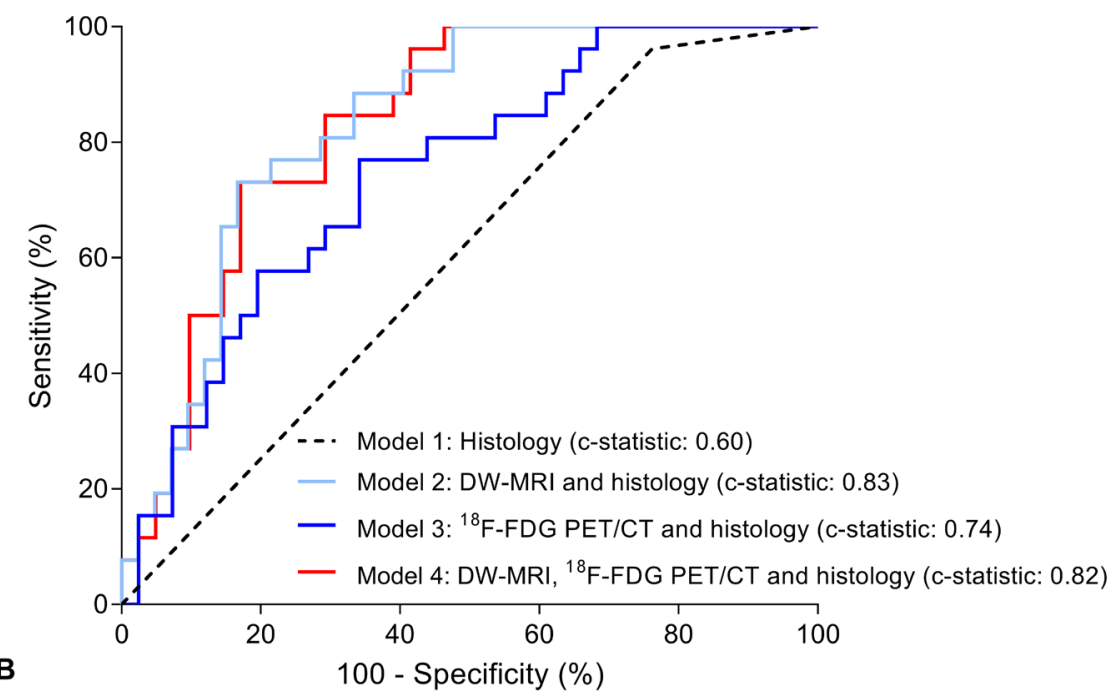

Figure 3. Receiver-operating-characteristic curve analysis for the regression models with DW-MRI and ${ }^{18} \mathrm{~F}-\mathrm{FDG}$ PET/CT parameters, as well as histopathological tumor type, for discriminating between pathologic complete response (TRG 1) and non-pathologic complete response (TRG 2-4) patients (A) as well as between good responders (TRG 1-2, GR) and poor responders (TRG 3-4, non-GR) (B). 


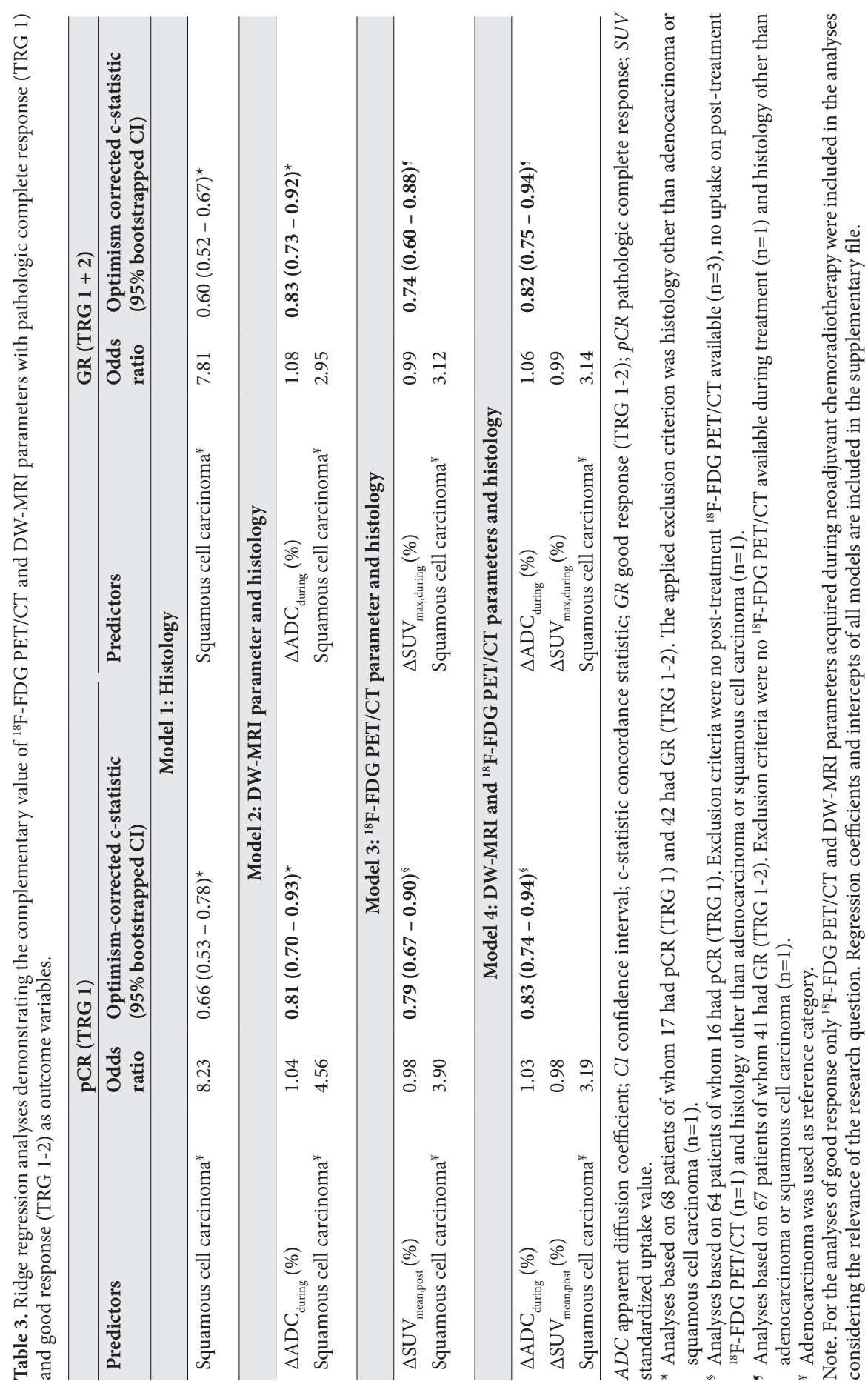


Table 4. Multivariable Cox regression analyses for overall survival (OS) and disease free survival (DFS) based on the model with DW-MRI and ${ }^{18}$ F-FDG PET/CT parameters and histology.

\begin{tabular}{llc}
\hline & Predictors & Hazard ratio (95\% CI) \\
\hline Overall survival $^{*}$ & $\Delta \mathrm{ADC}_{\text {during }}(\%)$ & $0.98(0.95-1.01)$ \\
& $\Delta \mathrm{SUV}_{\text {mean,post }}(\%)$ & $0.99(0.97-1.01)$ \\
Disease free survival $^{\S}$ & $\mathrm{Squamous} \mathrm{cell} \mathrm{carcinoma}^{\ddagger}$ & $0.67(0.21-2.16)$ \\
& $\Delta \mathrm{ADC}_{\text {during }}(\%)$ & $0.98(0.95-1.01)$ \\
& $\Delta \mathrm{SUV}_{\text {mean,post }}(\%)$ & $1.00(0.98-1.01)$ \\
& Squamous cell carcinoma $^{¥}$ & $0.65(0.17-2.44)$ \\
\hline
\end{tabular}

$A D C$ apparent diffusion coefficient; $C I$ confidence interval; SUV standardized uptake value; TLG tumor lesion glycolysis; TRG tumor regression grade

* Analyses based on 63 patients of whom 27 deceased during follow-up. The applied exclusion criteria were no post-treatment ${ }^{18} \mathrm{~F}$-FDG PET/CT available $(\mathrm{n}=3)$, no uptake on post-treatment ${ }^{18} \mathrm{~F}-\mathrm{FDG}$ PET/CT $(\mathrm{n}=1)$, histology other than adenocarcinoma or squamous cell carcinoma $(n=1)$ and incomplete follow-up data $(n=1)$.

s Analyses based on 57 patients of whom 24 had disease recurrence during follow-up. The applied exclusion criteria were no post-treatment ${ }^{18} \mathrm{~F}$-FDG PET/CT available $(\mathrm{n}=3)$, no uptake on post-treatment ${ }^{18} \mathrm{~F}$-FDG PET/ CT $(n=1)$, histology other than adenocarcinoma or squamous cell carcinoma $(n=1)$, incomplete follow-up data $(n=5)$ and censored before earliest event $(n=1)$.

₹ Adenocarcinoma was used as reference category.

\section{Survival}

The results of the multivariable Cox regression analysis for OS and DFS including the parameters from the most accurate model for PCR prediction are depicted in Table 4. None of the included imaging parameters $\left(\Delta \mathrm{ADC}_{\text {during }}\right.$ and $\left.\Delta \mathrm{SUV}_{\text {mean,post }}\right)$, nor histology was significantly associated with OS or DFS, as all 95\% CI of the hazard ratio's crossed the null hypothesis.

\section{Discussion}

This international multicenter, prospective study was designed to assess the predictive value of quantitative changes on ${ }^{18} \mathrm{~F}$-FDG PET/CT and DW-MRI scans acquired during and after nCRT in patients with esophageal cancer. Changes in ${ }^{18} \mathrm{~F}-\mathrm{FDG}$ PET/CT parameters after nCRT $\left(\triangle \mathrm{SUV}_{\text {mean,post }}\right.$ and $\left.\Delta \mathrm{TLG}_{\text {post }}\right)$, as well as changes in DW-MRI parameters during nCRT $\left(\triangle \mathrm{ADC}_{\text {during }}\right)$ demonstrate to discriminate well between pathologic complete responders (TRG 1) and non-pCR (TRG 2-4) in esophageal cancer. Moreover, ${ }^{18} \mathrm{~F}-\mathrm{FDG}$ PET/CT and DW-MRI might be of complementary value in the assessment of histopathological response. However, the prediction model including both imaging features did not correspond with OS or DFS in a subsequent experimental analysis on long-term clinical outcomes.

This study provides encouraging results for the potential value of multimodal imaging in discrimination of patients likely to have pCR to nCRT. This is of important clinical value, as accurate response prediction would enable physicians to individualize and adapt therapy 
for each individual patient. Considering the postoperative morbidity and mortality associated with surgery, and the effect of surgery on quality of life, an organ-sparing approach might improve outcomes for patients with pCR. ${ }^{31-33}$ Whereas in patients with a poor response to nCRT, alternative treatment strategies could be explored such as intensification or additional neoadjuvant treatment, or ineffective therapy could be stopped. For this latter purpose, a model was developed based on changes in ${ }^{18} \mathrm{~F}-\mathrm{FDG}$ PET/CT and DW-MRI parameters during nCRT, with GR (TRG 1-2) as outcome variable, demonstrating a high predictive performance of a DW-MRI parameter and tumor histology (c-statistic $0.83)$.

Until now, most imaging studies in esophageal response prediction have focused on a single modality. Two meta-analyses on the value of ${ }^{18} \mathrm{~F}-\mathrm{FDG}$ PET/CT suggested that the decrease in mean or maximum metabolic activity within the first two weeks of nCRT are so far the best available predictor of treatment response. ${ }^{14,19}$ However, the conclusion of these metaanalyses, including a more recent extensive analysis, is that the discriminatory ability of ${ }^{18} \mathrm{~F}-\mathrm{FDG}$ PET/CT was insufficient for clinical decision-making. ${ }^{20}$ The results of this study are consistent with these findings, with a maximum c-statistic of 0.74 for an ${ }^{18} \mathrm{~F}-\mathrm{FDG}$ PET/ $\mathrm{CT}$ imaging parameter $\left(\triangle \mathrm{SUV}_{\text {mean }}\right.$ after nCRT). Our study results are also consistent with the findings of more recent studies that showed no significant associations between relative changes ${ }^{18} \mathrm{~F}-\mathrm{FDG}$ PET/CT imaging parameters for $\mathrm{pCR}$, or only for $\triangle \mathrm{TLG}_{\mathrm{during}}{ }^{17,27}$ A pilot study using DW-MRI reported that the change in the tumor ADC during the first 2-3 weeks of nCRT for esophageal cancer seemed highly predictive for $\mathrm{pCR} .{ }^{16}$ These findings have recently been externally validated in another single-center pilot study ${ }^{17}$, but have not yet been validated in a larger cohort. The current, larger and multicenter study confirms these findings, reporting the highest c-statistic for prediction of $\mathrm{pCR}$ and GR for $\triangle \mathrm{ADC}_{\text {during }}$ (0.77 and 0.84 , respectively). Addition of ${ }^{18} \mathrm{~F}-\mathrm{FDG}$ PET/CT parameters to the DW-MRI model raised the ability to discriminate between pCR (TRG 1) and non-pCR (TRG 2-4) in $83 \%$ of the patients (c-statistic: 0.83 ).

As an illustration, the patient with an squamous cell carcinoma - whose scans are depicted in Figure 2 - and a $\triangle \mathrm{ADC}_{\text {during }}$ of $25 \%$ and $\triangle \mathrm{SUV}_{\text {mean,post }}$ of $-79 \%$, has a predicted probability of 0.69 for a pCR as based on the model including ${ }^{18} \mathrm{~F}-\mathrm{FDG}$ PET/CT, DW-MRI parameters and histology. A patient with an adenocarcinoma and $\triangle \mathrm{ADC}_{\text {during }}$ of $10 \%$ and $\triangle \mathrm{SUV}_{\text {mean,post }}$ of $-20 \%$, would have only had a predicted probability 0.12 for pCR based on the model. It is unclear yet what predictive power is required for clinical decision making as this might vary between patients. It could well be argued that for high risk surgical patients with significant comorbidities, a lower accuracy of a predicted pCR might be deemed sufficient to postpone standard esophagectomy, whereas for younger, low risk surgical patients, a higher accuracy might be desired. Other considerations to be taken into account are how many patients will require salvage surgery, and how many patients will have missed an 
opportunity for cure because surgery was initially avoided, as death of locoregional disease will be considered as the ultimate failure of therapy. Eventually, future improvement in prognostication will likely result in stratification of patients by risk of surgery balanced with the predicted probability of pCR.

Other strategies to identify good responders are currently subject of research in multiple trials. The recently published preSANO study focused on accurate detection of locoregional residual disease rather than pathologic complete response, and reported favorable results for response evaluations using EUS, bite-on-bite biopsies, fine-needle aspiration of suspicious lymph nodes and ${ }^{18} \mathrm{~F}-\mathrm{FDG}$ PET/CT. ${ }^{34}$ Furthermore, it has been recently recognized that the combination of clinical and imaging parameters with molecular biomarkers might be the key to success. Besides molecular markers - such as ALDH1 and GLI1/HH - which might predict response before nCRT, other molecular markers, such as circulating tumor DNA (ctDNA), that evaluate response after completion of nCRT should be further explored. ${ }^{35,36} \mathrm{~A}$ large Dutch multicenter study is currently investigating the additional value of ctDNA to ${ }^{18} \mathrm{~F}-\mathrm{FDG}$ PET/CT and MRI for accurate pCR prediction (PRIDE study). ${ }^{37}$

Since ${ }^{18} \mathrm{~F}-\mathrm{FDG}$ PET/CT and DW-MRI scanning during and after completion of nCRT are currently not part of standard imaging evaluation, the predictive value of multimodal imaging evaluation should be considered in light of the associated costs and physical burden to the patients of repeated imaging procedures. Previous studies have demonstrated that ${ }^{18} \mathrm{~F}-\mathrm{FDG} \mathrm{PET} / \mathrm{CT}$ and MRI are generally well-tolerated imaging procedures for the assessment of response to treatment in esophageal cancer patients. ${ }^{38}$ Furthermore, longterm treatment outcomes (survival and long-term quality of life) appear to outweigh shortterm attributes (short-term quality of life and burden of surveillance examinations with endoscopy and PET/CT) in another study. ${ }^{39}$ In this perspective, further investigation of multimodal strategies for reliable response prediction to nCRT seems justified.

Several challenges exist before routine ${ }^{18} \mathrm{~F}-\mathrm{FDG}$ PET/CT and DW-MRI scanning for treatment response assessment can be implemented for clinical practice. First, quantitative imaging values such as $\mathrm{SUV}_{\max }$ from ${ }^{18} \mathrm{~F}$-FDG PET/CT imaging are influenced by vendor specific characteristics, which differ between the scanner platforms. However, since our study only included relative changes in the analyses, the impact of these differences on the results is limited. Standardization of the DW-MRI protocols is more challenging than for ${ }^{18} \mathrm{~F}-\mathrm{FDG}$ PET/CT protocols, as they are comprised of more nuanced sequences that are more likely to differ between institutions. ${ }^{40,41}$ The MRI scan protocol applied in the current study was clinically developed specifically for esophageal cancer patients, and extensive efforts have been undertaken to synchronize the protocols before initiation of the study. Even though standardization of MRI protocols appears to be challenging, previous studies have found similar changes in mean tumor ADC during nCRT in esophageal cancer to be 
predictive of pCR, despite major differences in scanner platforms (3.0T versus 1.5T, GE versus Philips). ${ }^{16,17}$ Furthermore, our study included both scanner types and found consistent results with the earlier studies.

Second, it became apparent during the assessment of the DW-MRI scans in our study that there is still room for improvement in the quality of these scans. The thorax is a challenging region for MR imaging, given the differences in magnetic susceptibility between the organs of the thorax (e.g., pulmonary parenchyma, vertebrae and ribs), as well as the effects of cardiac and respiratory motion. ${ }^{42}$ As such, MR imaging in the thoracic region remains under continuous technical development, and better imaging quality may have the potential to further improve the performance of DW-MRI for treatment response assessment. ${ }^{43}$

Third, even though semi-automatic contouring methods were used, differences between contouring techniques and inter-reader variability could still be an issue. However, the reproducibility of ADC measurements between readers has been studied previously, and was considered reproducible among 5 readers in a relatively small dataset of 20 esophageal cancer patients. ${ }^{17}$ These high correlations were achieved when ADC values were assessed with whole volume tumor ADC measurements, as in our study. As semi-automatic tumor delineation on ${ }^{18} \mathrm{~F}$-FDG PET/CT and DW-MRI scans remains a time-consuming process, the clinical applicability of ${ }^{18} \mathrm{~F}-\mathrm{FDG}$ PET/CT and DW-MRI for response assessment might benefit from a validated fully automatic contouring tool.

Fourth, the prediction model including both imaging features that provided good performance for prediction of PCR did not correspond with OS or DFS in a subsequent experimental analysis. We believe that the survival analyses in the current study should be interpreted with caution, as these survival analyses were not predefined and hence may suffer from a lack of power. Also, it is essential to first develop a clinically useful prediction model to identify complete responders, before drawing any conclusions regarding survival. Especially since subsequent analyses with survival will only make sense if identification of pCR is accurate and treatment is altered based on these predictions, as the burden of 3 additional MRI's and 1 additional PET/CT (as in the current study) can only be justified if this results in treatment alterations, and not just for prognostic purposes.

Lastly, limitations of the current study include the absence of an external validation cohort to validate our results and the various nCRT regimens applied. However, consistent findings in a slightly heterogeneous, multicenter study population confers external validity to the results of the study (Supplementary Table 1). Other strengths of the current study include the availability of histopathological evaluation as a reference standard, instead of surrogate endpoints such as radiological response criteria (RECIST), and the inclusion of histologic subtypes in the multivariable analyses.

In conclusion, our study shows that quantitative ADC changes from baseline to interim DW-MRI scans and SUV ${ }_{\text {mean }}$ changes from baseline to follow-up ${ }^{18} \mathrm{~F}-\mathrm{FDG}$ PET/CT scans 
can help identify pCR to nCRT in esophageal cancer patients. However, additional larger prospective studies, as well as other combined multimodal approaches are needed to validate these results, especially regarding the potentially complementary value of ${ }^{18} \mathrm{~F}$-FDG PET/CT and DW-MRI imaging parameters. 


\section{References}

1. Lordick F, Mariette C, Haustermans K, et al. Oesophageal cancer: ESMO Clinical Practice Guidelines for diagnosis, treatment and follow-up. Ann Oncol. 2016;27:v50-v57.

2. Sjoquist KM, Burmeister BH, Smithers BM, et al. Survival after neoadjuvant chemotherapy or chemoradiotherapy for resectable oesophageal carcinoma: an updated meta-analysis. Lancet Oncol. 2011;12:681-92.

3. Shapiro J, Lanschot JJB Van, Hulshof MCCM, et al. Neoadjuvant chemoradiotherapy plus surgery versus surgery alone for oesophageal or junctional cancer (CROSS): long-term results of a randomised controlled trial. Lancet Oncol. 2015;16:1090-1098.

4. van Hagen P, Hulshof MCCM, van Lanschot JJB, et al. Preoperative Chemoradiotherapy for Esophageal or Junctional Cancer. N Engl J Med. 2012;366:2074-2084.

5. Steffen T, Dietrich D, Schnider A, et al. Recurrence Patterns and Long-Term Results After Induction Chemotherapy, Chemoradiotherapy, and Curative Surgery in Patients With Locally Advanced Esophageal Cancer. Ann Surg. 2019;269:83-87.

6. Noordman BJ, Wijnhoven BPL, Lagarde SM, et al. Active surveillance in clinically complete responders after neoadjuvant chemoradiotherapy for esophageal or junctional cancer. Dis Esophagus. 2017;30:1-8.

7. Westerterp M, van Westreenen HL, Reitsma JB, et al. Esophageal Cancer: CT, Endoscopic US, and FDG PET for Assessment of Response to Neoadjuvant Therapy-Systematic Review. Radiology. 2005;236:841851.

8. van Rossum PSN, Goense L, Meziani J, et al. Endoscopic biopsy and EUS for the detection of pathologic complete response after neoadjuvant chemoradiotherapy in esophageal cancer: a systematic review and meta-analysis. Gastrointest Endosc. 2016;83:866-879.

9. Wang L, Liu L, Han C, et al. The diffusion-weighted magnetic resonance imaging (DWI) predicts the early response of esophageal squamous cell carcinoma to concurrent chemoradiotherapy. Radiother Oncol. 2016;121:246-251.

10. Yip C, Cook GJR, Landau DB, et al. Performance of different imaging modalities in assessment of response to neoadjuvant therapy in primary esophageal cancer. Dis Esophagus. 2016;29:116-130.

11. Ngamruengphong S, Sharma VK, Nguyen B, et al. Assessment of response to neoadjuvant therapy in esophageal cancer: an updated systematic review of diagnostic accuracy of endoscopic ultrasonography and fluorodeoxyglucose positron emission tomography. Dis Esophagus. 2010;23:216-31.

12. Koh D-M, Collins DJ. Diffusion-Weighted MRI in the Body: Applications and Challenges in Oncology. Am J Roentgenol. 2007;188:1622-1635.

13. Lambrecht M, Vandecaveye V, De Keyzer F, et al. Value of Diffusion-Weighted Magnetic Resonance Imaging for Prediction and Early Assessment of Response to Neoadjuvant Radiochemotherapy in Rectal Cancer: Preliminary Results. Int J Radiat Oncol. 2012;82:863-870.

14. Kwee RM. Prediction of Tumor Response to Neoadjuvant Therapy in Patients with Esophageal Cancer with Use of ${ }^{18}$ F FDG PET: A Systematic Review. Radiology. 2010;254:707-717.

15. Aoyagi T, Shuto K, Okazumi S, et al. Apparent diffusion coefficient values measured by diffusion-weighted imaging predict chemoradiotherapeutic effect for advanced esophageal cancer. Dig Surg. 2011;28:252-7.

16. van Rossum PSN, van Lier ALHMW, van Vulpen M, et al. Diffusion-weighted magnetic resonance imaging for the prediction of pathologic response to neoadjuvant chemoradiotherapy in esophageal cancer. Radiother Oncol. 2015;115:163-170.

17. Fang P, Musall BC, Son JB, et al. Multimodal Imaging of Pathologic Response to Chemoradiation in Esophageal Cancer. Int J Radiat Oncol Biol Phys. 2018;102:996-1001.

18. De Cobelli F, Giganti F, Orsenigo E, et al. Apparent diffusion coefficient modifications in assessing gastrooesophageal cancer response to neoadjuvant treatment: Comparison with tumour regression grade at histology. Eur Radiol. 2013;23:2165-2174.

19. Chen Y-M, Pan X, Tong L-J, et al. Can 18F-fluorodeoxyglucose positron emission tomography predict responses to neoadjuvant therapy in oesophageal cancer patients? A meta-analysis. Nucl Med Commun. 2011;32:1005-10.

20. van Rossum PSN, Fried D V, Zhang L, et al. The Incremental Value of Subjective and Quantitative Assessment of 18F-FDG PET for the Prediction of Pathologic Complete Response to Preoperative Chemoradiotherapy in Esophageal Cancer. J Nucl Med. 2016;57:691-700. 
21. Rice TW, Blackstone EH, Rusch VW. 7th Edition of the AJCC Cancer Staging Manual: Esophagus and Esophagogastric Junction. Ann Surg Oncol. 2010;17:1721-1724.

22. Chirieac LR, Swisher SG, Ajani JA, et al. Posttherapy pathologic stage predicts survival in patients with esophageal carcinoma receiving preoperative chemoradiation. Cancer. 2005;103:1347-1355.

23. Roedl JB, Harisinghani MG, Colen RR, et al. Assessment of treatment response and recurrence in esophageal carcinoma based on tumor length and standardized uptake value on positron emission tomography-computed tomography. Ann Thorac Surg. 2008;86:1131-8.

24. Li Q-W, Qiu B, Wang B, et al. Prediction of pathologic responders to neoadjuvant chemoradiotherapy by diffusion-weighted magnetic resonance imaging in locally advanced esophageal squamous cell carcinoma: a prospective study. Dis Esophagus. 2018;31(2).

25. Tan S, Kligerman S, Chen W, et al. Spatial-Temporal [18F]FDG-PET Features for Predicting Pathologic Response of Esophageal Cancer to Neoadjuvant Chemoradiation Therapy. Int J Radiat Oncol. 2013;85:13751382.

26. Beukinga RJ, Hulshoff JB, Mul VEM, et al. Prediction of Response to Neoadjuvant Chemotherapy and Radiation Therapy with Baseline and Restaging 18 F-FDG PET Imaging Biomarkers in Patients with Esophageal Cancer. Radiology. 2018;287:983-992.

27. Elimova E, Wang X, Etchebehere E, et al. 18-fluorodeoxy-glucose positron emission computed tomography as predictive of response after chemoradiation in oesophageal cancer patients. Eur J Cancer. 2015;51:2545-2552.

28. van Heijl M, Omloo JM, van Berge Henegouwen MI, et al. Fluorodeoxyglucose Positron Emission Tomography for Evaluating Early Response During Neoadjuvant Chemoradiotherapy in Patients With Potentially Curable Esophageal Cancer. Ann Surg. 2011;253:56-63.

29. Benjamini Y, Hochberg Y. Controlling the False Discovery Rate: A Practical and Powerful Approach to Multiple Testing. J R Stat Soc. 1995;57:289-300.

30. Pavlou M, Ambler G, Seaman SR, et al. How to develop a more accurate risk prediction model when there are few events. BMJ. 2015;351:h3868.

31. Low DE, Kuppusamy MK, Alderson D, et al. Benchmarking Complications Associated with Esophagectomy. Ann Surg. 2019;269:291-298.

32. Kauppila JH, Johar A, Lagergren P. Postoperative Complications and Health-related Quality of Life 10 Years After Esophageal Cancer Surgery. Ann Surg. Epub ahead of print July 10, 2018.

33. Noordman BJ, Van Klaveren D, van Berge Henegouwen MI, et al. Impact of Surgical Approach on Longterm Survival in Esophageal Adenocarcinoma Patients With or Without Neoadjuvant Chemoradiotherapy. Ann Surg. 2017;267:892-897.

34. Noordman BJ, Spaander MCW, Valkema R, et al. Detection of residual disease after neoadjuvant chemoradiotherapy for oesophageal cancer (preSANO): a prospective multicentre, diagnostic cohort study. Lancet Oncol. 2018;19:965-974.

35. Creemers A, Ebbing EA, Pelgrim TC, et al. A systematic review and meta-analysis of prognostic biomarkers in resectable esophageal adenocarcinomas. Sci Rep. 2018;8:13281.

36. Ajani JA, Bhutani MS, Swisher SG. Oesophageal preservation in locally advanced oesophageal cancer. Lancet Oncol. 2018;19:e430.

37. Borggreve AS, Mook S, Verheij M, et al. Preoperative image-guided identification of response to neoadjuvant chemoradiotherapy in esophageal cancer (PRIDE): a multicenter observational study. $B M C$ Cancer. 2018;18:1006.

38. Goense L, Borggreve AS, Heethuis SE, et al. Patient perspectives on repeated MRI and PET/CT examinations during neoadjuvant treatment of oesophageal cancer. Br J Radiol. 2018;91:20170710.

39. Noordman BJ, de Bekker-Grob EW, Coene PPLO, et al. Patients' preferences for treatment after neoadjuvant chemoradiotherapy for oesophageal cancer. Br J Surg. 2018;105:1630-1638.

40. Verma V, Simone CB, Krishnan S, et al. The Rise of Radiomics and Implications for Oncologic Management. J Natl Cancer Inst. 2017;109(7).

41. deSouza NM. Diffusion-weighted MRI in Multicenter Trials of Breast Cancer: A Useful Measure of Tumor Response? Radiology. 2018;289:628-629.

42. Andre JB, Bammer R. Advanced diffusion-weighted magnetic resonance imaging techniques of the human spinal cord. Top Magn Reson imaging. 2010;21:367-78.

43. van Rossum PS, van Lier AL, Lips IM, et al. Imaging of oesophageal cancer with FDG-PET/CT and MRI. Clin Radiol. 2015;70:81-95. 


\section{SUPPLEMENTARY MATERIAL}

\section{Methods}

\section{Exclusion criteria}

Exclusion criteria included an age of $<18$ years, previous treatment with thoracic surgery or thoracic radiotherapy, and contraindications for ${ }^{18} \mathrm{~F}-\mathrm{FDG}$ PET/CT or MRI. The diagnostic work-up consisted of an endoscopy with biopsy for diagnosis, as well as EUS and integrated ${ }^{18}$ F-FDG PET/CT. In addition, patients who were initially included in the study but eventually did not undergo surgery were excluded from further analyses.

\section{Survival}

Survival data was completed for all but 1 patient $(n=68)$ at least up to 18 months after date of surgery (median [IQR] time to censoring: 37 months [33 - 49 months]). Data on disease recurrence and disease free survival (DFS) was completed for all but 5 patients $(n=63)$. Disease recurrence was defined as local or distant recurrence, either based on imaging or histopathological assessment.

\section{${ }^{18}$ F-FDG PET/CT scan parameters}

The timing of the ${ }^{18} \mathrm{~F}-\mathrm{FDG}$ PET/CT scan in the second or third week of treatment was based on previous studies which demonstrated a potentially superior accuracy at this time point as compared to pre-treatment and post-treatment scanning only. ${ }^{1,2}$ The ${ }^{18} \mathrm{~F}$-FDG PET examinations were performed on dedicated PET/CT systems. Patients were instructed to fast for at least 6 hours before ${ }^{18} \mathrm{~F}-\mathrm{FDG}$ PET and a glucose level within the normal range (80-120 mg/dl) was confirmed. Before ${ }^{18}$ F-FDG PET, a CT scan without contrast agent was acquired for attenuation correction purposes. ${ }^{18} \mathrm{~F}$-FDG PET scans were acquired 60-90 minutes after administration of ${ }^{18} \mathrm{~F}-\mathrm{FDG}$ with a dose ranging between 190-370 MBq, in three-dimensional (3D) acquisition mode at 2-5 minutes per bed position.

\section{DW-MRI scan parameters}

The DW-MRI examinations were either performed on a 1.5T (UMC Utrecht and NKI-AVL; Achieva, Philips Medical Systems, Best, The Netherlands) or on a 3.0T scanner (MDACC; Discovery MR750, GE Healthcare, Milwaukee, Wisconsin, USA). Transverse diffusionweighted images were obtained with free breathing and using 3 different $b$-values $(b=0$, 200 and $800 \mathrm{~s} / \mathrm{mm}^{2}$ ). 


\section{Image analysis - tumor delineations}

${ }^{18} \mathrm{~F}-\mathrm{FDG}$ PET/CT imaging analysis, including primary tumor delineation and calculation of metabolic and volumetric parameters, was performed using commercially available software (MIM Software, Cleveland, Ohio, USA). The primary tumor volume was defined as the volume of interest (VOI) and contoured using a semi-automatic gradient-based delineation method - which has been validated in a multi-observer study reporting superior accuracy, consistency and robustness compared with manual and threshold methods ${ }^{3}-$ followed by manual editing by two readers.

DW-MRI analysis was performed using an imaging analysis software package (ImageI). ${ }^{4}$ The primary tumor - excluding the lumen - was delineated on the DW-MRI scans with a b-value of $200 \mathrm{~s} / \mathrm{mm}^{2}$ using semi-automatic contouring, allowing for manual editing by one reader. ${ }^{4}$ Contouring of the tumor was performed conservatively to avoid the edges of the tumor boundaries, as ADC values in the periphery of the tumor may be unreliable due to motion or other image distortions. The DW-MRI scans with b-values of 0,200 , and 800 $\mathrm{s} / \mathrm{mm}^{2}$ were fitted with a mono-exponential model to generate quantitative ADC maps for each slice..$^{5-7}$

\section{Statistical analysis}

Clinical characteristics that potentially predict response were pre-specified based on previous literature (i.e., clinical T status, histologic subtype, neoadjuvant chemoradiotherapy regimen and time interval from nCRT to surgery) and were compared between patients with pCR (TRG 1) and non-pCR patients (TRG 2-4), and between good responders (TRG $1-2, \mathrm{GR}$ ) and poor responders (TRG 3-4, non-GR) based on the $\chi^{2}$ or Fisher's exact test for categorical variables and the Mann-Whitney $U$ test for continuous variables.

The relative changes of the ${ }^{18} \mathrm{~F}-\mathrm{FDG}$ PET/CT and DW-MRI parameters were also compared between these patient groups using the Mann-Whitney $U$ test to validate findings of previous pilot-studies. ${ }^{5,7-12}$ Benjamini-Hochberg corrections were applied to adjust for multiple comparisons and therewith minimizing the false discovery rate. ${ }^{13}$ The ability of single modality ${ }^{18} \mathrm{~F}-\mathrm{FDG}$ PET/CT and DW-MRI parameters to discriminate between different pathologic response groups was quantified using the area under the receiver operating characteristic (ROC) curve (c-statistic).

Secondly, the complementary value of ${ }^{18} \mathrm{~F}-\mathrm{FDG}$ PET/CT and DW-MRI parameters was assessed using multivariable penalized Ridge regression models to reduce model overfitting in a situation with few events per variable. ${ }^{14}$ The applied overall penalty $\left(\lambda_{\min }\right)$ represents the minimum mean cross-validated error, which was obtained using 10 -fold cross validation. Optimism-corrected c-statistics of the Ridge regression models were obtained by bootstrap resampling. ${ }^{15}$ The original dataset was a 1,000 times resampled with replacement to obtain a dataset of the same size. All models were then fitted to the 1,000 
bootstrap samples. Each fitted model was then applied both to the resampled dataset from which it was generated, and to the original dataset. The optimism corrected c-statistic was calculated as the original c-statistic minus the optimism, which was calculated as the difference between the c-statistic on the original dataset and the resampled dataset. Also, bootstrapping allowed for reconstruction of 95\% confidence intervals (CI) around the c-statistic. The global fit of the models was compared using Akaike Information Criterion (AIC), with the lowest AIC representing the best fit. ${ }^{16,17}$ Model calibration of the models was evaluated by visual inspection of the model calibration plots. ${ }^{17}$ Variables to be entered in the penalized regression models with pCR or GR as outcome were histopathological tumor type - which an important factor to impact pathologic response to nCRT based on previous literature - and the ${ }^{18} \mathrm{~F}-\mathrm{FDG}$ PET/CT and DW-MRI parameter with the highest c-statistic in univariable analyses.

Separate analyses were performed on pCR (TRG 1) versus non-pCR (TRG 2-4), and GR (TRG 1-2) versus non-GR (TRG 3-4). The first analysis aimed at aiding clinical decisionmaking regarding omission of surgery in anticipated complete responders, whereas the second analysis was deemed as more relevant for potentially modifying or discontinuing nCRT early during treatment. For the latter purpose, only ${ }^{18} \mathrm{~F}-\mathrm{FDG}$ PET/CT and DW-MRI parameters during nCRT were taken into account, as knowledge of response after completion of nCRT would be irrelevant for modification of nCRT.

In order to evaluate whether the best performing model for $\mathrm{PCR}$ prediction based on the aforementioned analyses correlate with overall survival (OS) and disease free survival (DFS), multivariable Cox regression analyses were performed with estimation of hazard ratio's (HR) and 95\% CI for the included predictors.

All statistical analyses were performed using R 3.1.2 open-source software ('pROC', 'glmnet', 'boot' and 'rms' packages, http://www.R-project.org). A p-value of $<0.05$ was considered statistically significant. 


\section{References}

1. Chen Y-M, Pan X, Tong L-J, et al. Can 18F-fluorodeoxyglucose positron emission tomography predict responses to neoadjuvant therapy in oesophageal cancer patients? A meta-analysis. Nucl Med Commun. 2011;32:1005-10.

2. Ott K, Weber WA, Lordick F, et al. Metabolic imaging predicts response, survival, and recurrence in adenocarcinomas of the esophagogastric junction. J Clin Oncol. 2006;24:4692-8.

3. Werner-Wasik M, Nelson AD, Choi W, et al. What is the best way to contour lung tumors on PET scans? Multiobserver validation of a gradient-based method using a NSCLC digital PET phantom. Int J Radiat Oncol Biol Phys. 2012;82:1164-71.

4. Musall B. Quantitative DWI as an Early Imaging Biomarker of the Response to Chemoradiation in Esophageal Cancer Available from: https://digitalcommons.library.tmc.edu/utgsbs_dissertations/805. 2017. Accessed September 4, 2018.

5. Fang P, Musall BC, Son JB, et al. Multimodal Imaging of Pathologic Response to Chemoradiation in Esophageal Cancer. Int J Radiat Oncol Biol Phys. 2018;102:996-1001.

6. Padhani AR, Liu G, Koh DM, et al. Diffusion-weighted magnetic resonance imaging as a cancer biomarker: consensus and recommendations. Neoplasia. 2009;11:102-25.

7. van Rossum PSN, van Lier ALHMW, van Vulpen M, et al. Diffusion-weighted magnetic resonance imaging for the prediction of pathologic response to neoadjuvant chemoradiotherapy in esophageal cancer. Radiother Oncol. 2015;115:163-170.

8. van Rossum PSN, Fried D V, Zhang L, et al. The Incremental Value of Subjective and Quantitative Assessment of 18F-FDG PET for the Prediction of Pathologic Complete Response to Preoperative Chemoradiotherapy in Esophageal Cancer. J Nucl Med. 2016;57:691-700.

9. Li Q-W, Qiu B, Wang B, et al. Prediction of pathologic responders to neoadjuvant chemoradiotherapy by diffusion-weighted magnetic resonance imaging in locally advanced esophageal squamous cell carcinoma: a prospective study. Dis Esophagus.;31.

10. Tan S, Kligerman S, Chen W, et al. Spatial-Temporal [18F]FDG-PET Features for Predicting Pathologic Response of Esophageal Cancer to Neoadjuvant Chemoradiation Therapy. Int J Radiat Oncol. 2013;85:13751382 .

11. Beukinga RJ, Hulshoff JB, Mul VEM, et al. Prediction of Response to Neoadjuvant Chemotherapy and Radiation Therapy with Baseline and Restaging 18 F-FDG PET Imaging Biomarkers in Patients with Esophageal Cancer. Radiology. 2018;287:983-992.

12. van Heijl M, Omloo JM, van Berge Henegouwen MI, et al. Fluorodeoxyglucose Positron Emission Tomography for Evaluating Early Response During Neoadjuvant Chemoradiotherapy in Patients With Potentially Curable Esophageal Cancer. Ann Surg. 2011;253:56-63.

13. Benjamini Y, Hochberg Y. Controlling the False Discovery Rate: A Practical and Powerful Approach to Multiple Testing. J R Stat Soc. 1995;57:289-300.

14. Pavlou M, Ambler G, Seaman SR, et al. How to develop a more accurate risk prediction model when there are few events. BMJ. 2015;351:h3868.

15. Steyerberg EW, Harrell FE, Borsboom GJJM, et al. Internal validation of predictive models: efficiency of some procedures for logistic regression analysis. J Clin Epidemiol. 2001;54:774-781.

16. Akaike H. Likelihood of a model and information criteria. J Econom. 1981;16:3-14.

17. Cook NR. Use and Misuse of the Receiver Operating Characteristic Curve in Risk Prediction. Circulation. 2007;115:928-935. 
Supplementary Table 1 . Tumor regression grades (TRG) per histologic subtype and per treatment regimen.

\begin{tabular}{|c|c|c|c|c|c|c|}
\hline Treatment regimen & Histologic subtypes & TRG 1 & TRG 2 & TRG 3 & TRG 4 & Total \\
\hline \multirow[t]{3}{*}{ Full cohort } & Adenocarcinoma & $10(17.5 \%)$ & $22(38.6 \%)$ & $19(33.3 \%)$ & $6(10.5 \%)$ & $57(100 \%)$ \\
\hline & $\begin{array}{l}\text { Squamous cell } \\
\text { carcinoma }\end{array}$ & $7(63.6 \%)$ & $3(27.3 \%)$ & $1(9.1 \%)$ & $0(0 \%)$ & $11(100 \%)$ \\
\hline & $\begin{array}{l}\text { Undifferentiated } \\
\text { large cell carcinoma }\end{array}$ & $1(100 \%)$ & $0(0 \%)$ & $0(0 \%)$ & $0(0 \%)$ & $1(100 \%)$ \\
\hline \multirow{3}{*}{$\begin{array}{l}\text { Carboplatin/ } \\
\text { paclitaxel + } 41.4 \text { Gy } \\
\text { (UMC Utrecht, } \\
\text { NKI-AVL) }\end{array}$} & Adenocarcinoma & $4(11.8 \%)$ & $12(35.3 \%)$ & $12(35.3 \%)$ & $6(17.6 \%)$ & $34(100 \%)$ \\
\hline & $\begin{array}{l}\text { Squamous cell } \\
\text { carcinoma }\end{array}$ & $6(75 \%)$ & $2(25 \%)$ & $0(0 \%)$ & $0(0 \%)$ & $8(100 \%)$ \\
\hline & $\begin{array}{l}\text { Undifferentiated } \\
\text { arge cell carcinoma }\end{array}$ & $1(100 \%)$ & $0(0 \%)$ & $0(0 \%)$ & $0(0 \%)$ & $1(100 \%)$ \\
\hline \multirow{2}{*}{$\begin{array}{l}\text { 5-fluorouracil-based } \\
+\mathbf{5 0 . 4} \text { Gy } \\
\text { (MDACC) }\end{array}$} & Adenocarcinoma & $6(26.1 \%)$ & $10(43.5 \%)$ & $7(30.4 \%)$ & $0(0 \%)$ & $23(100 \%)$ \\
\hline & $\begin{array}{l}\text { Squamous cell } \\
\text { carcinoma }\end{array}$ & $1(33.3 \%)$ & $1(33.3 \%)$ & $1(33.3 \%)$ & $0(0 \%)$ & $3(100 \%)$ \\
\hline
\end{tabular}

Data are numbers of patients, with row-based percentages in parentheses.

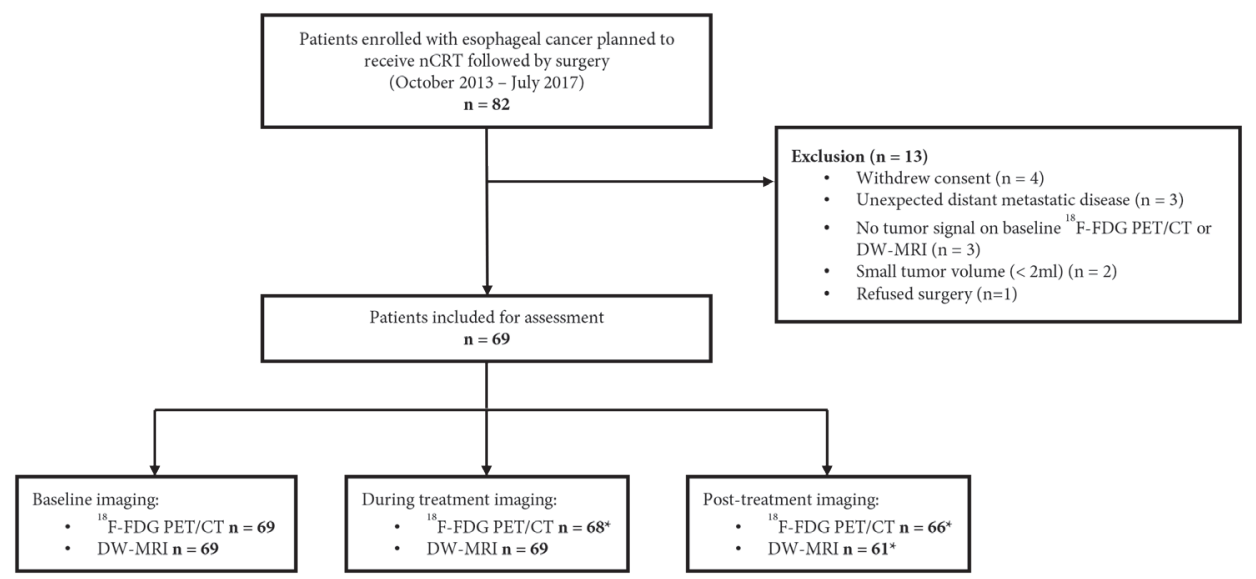

* Not available due to patients' refusal or an unexpectedly antedated surgical resection.

Supplementary Figure 1. Study profile. 
A. Model 1 (pCR)

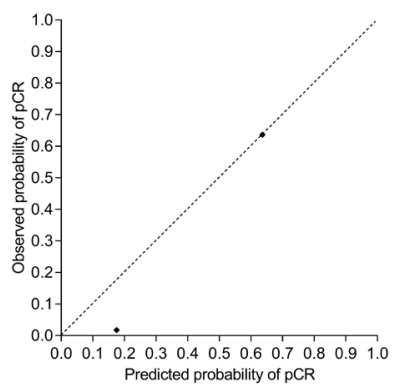

B. Model 2 ( $\mathrm{PCR}$ )

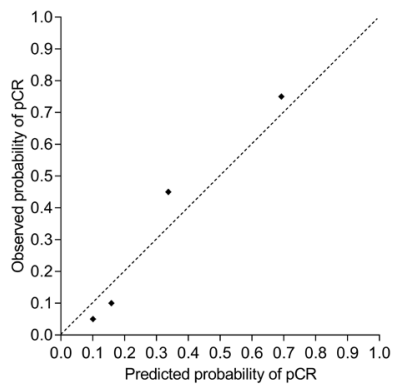

C. Model 3 (pCR)

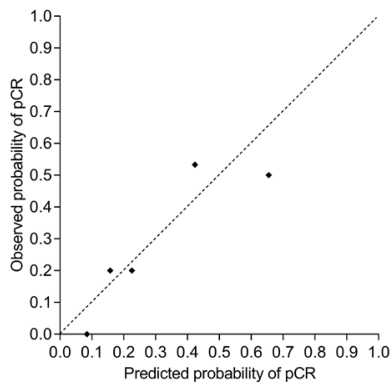

D. Model 4 (pCR)

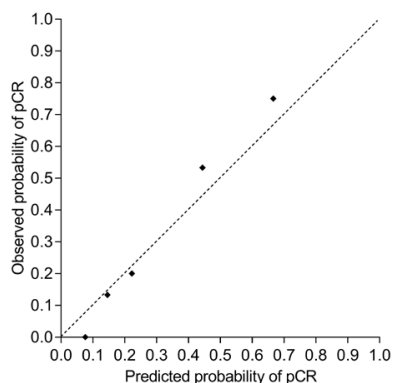

E. Model 1 (GR)

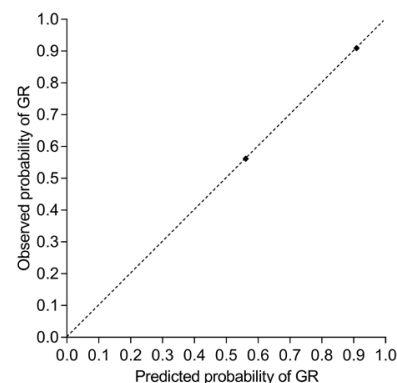

F. Model 2 (GR)

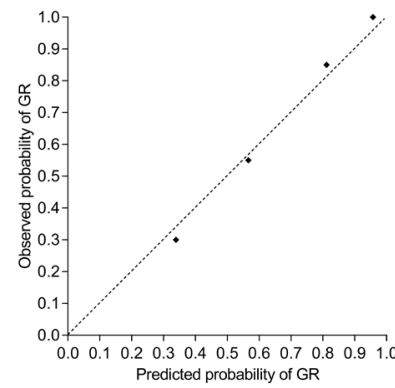

G. Model 3 (GR)

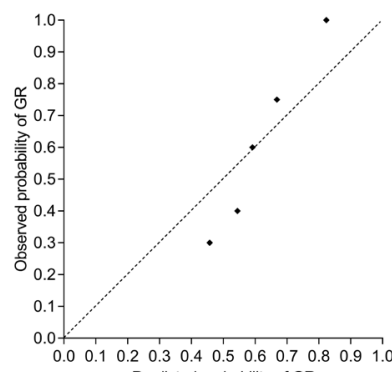

Predicted probability of GR

H. Model 4 (GR)

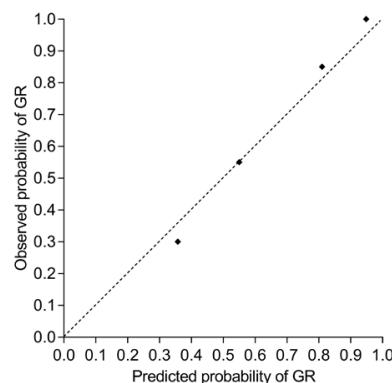

Supplementary Figure 2. Calibration plots of the penalized regression models as described in Table 3 for pathologic complete response (pCR) prediction (A-D) and good response (GR) prediction (E-H). 
Supplementary Table 2. Intercepts and regression coefficients for Ridge regression analyses on the complementary value of ${ }^{18} \mathrm{~F}-\mathrm{FDG}$ PET/CT and DW-MRI parameters with pathologic complete response (TRG 1) and good response (TRG 1-2) as outcome variables.

\begin{tabular}{|c|c|c|c|c|c|}
\hline \multicolumn{3}{|l|}{ pCR (TRG 1) } & \multicolumn{3}{|l|}{ GR (TRG 1 + 2) } \\
\hline Intercept and predictors & $\beta$ & AIC & Intercept and predictors & $\beta$ & AIC \\
\hline \multicolumn{6}{|c|}{ Model 1: Histology } \\
\hline Intercept & -1.55 & 71.36 & Intercept & 0.25 & 88.85 \\
\hline Squamous cell carcinoma ${ }^{¥}$ & 2.10 & & Squamous cell carcinoma ${ }^{*}$ & 2.06 & \\
\hline
\end{tabular}

\begin{tabular}{lccccc}
\hline \multicolumn{5}{c}{ Model 2: DW-MRI parameter and histology } \\
\hline Intercept & -2.20 & 64.73 & Intercept & -0.71 & 85.83 \\
$\Delta \mathrm{ADC}_{\text {during }}(\%)$ & 0.04 & $\Delta \mathrm{ADC}_{\text {during }}(\%)$ & 0.08 \\
Squamous cell carcinoma $^{¥}$ & 1.52 & Squamous cell carcinoma $^{¥}$ & 1.08
\end{tabular}

\begin{tabular}{lrllrl}
\hline \multicolumn{5}{c}{ Model 3: ${ }^{18}$ F-FDG PET/CT parameter and histology } \\
\hline Intercept & -2.51 & 61.32 & Intercept & 0.12 & 71.64 \\
$\Delta$ SUV $_{\text {mean,post }}(\%)$ & -0.02 & & $\Delta$ SUV $_{\text {max,during }}(\%)$ & -0.01 \\
Squamous cell carcinoma $^{\text {I }}$ & 1.36 & Squamous cell carcinoma $^{¥}$ & 1.14
\end{tabular}

\begin{tabular}{|c|c|c|c|c|c|}
\hline \multicolumn{6}{|c|}{ Model 4: DW-MRI and ${ }^{18}$ F-FDG PET/CT parameters and histology } \\
\hline Intercept & -2.68 & 61.13 & Intercept & -0.69 & 72.31 \\
\hline$\Delta \mathrm{ADC}_{\text {during }}(\%)$ & 0.03 & & $\Delta \mathrm{ADC}_{\text {during }}(\%)$ & 0.06 & \\
\hline$\Delta \mathrm{SUV}_{\text {mean,post }}(\%)$ & -0.02 & & $\Delta \mathrm{SUV}_{\text {max,during }}(\%)$ & -0.01 & \\
\hline Squamous cell carcinoma ${ }^{¥}$ & 1.16 & & Squamous cell carcinoma ${ }^{¥}$ & 1.14 & \\
\hline
\end{tabular}

$A D C$ apparent diffusion coefficient; AIC Akaike Information Criterion; GR good response (TRG 1-2); $p C R$ pathologic complete response; $S U V$ standardized uptake value

${ }^{¥}$ Adenocarcinoma was used as reference category. 


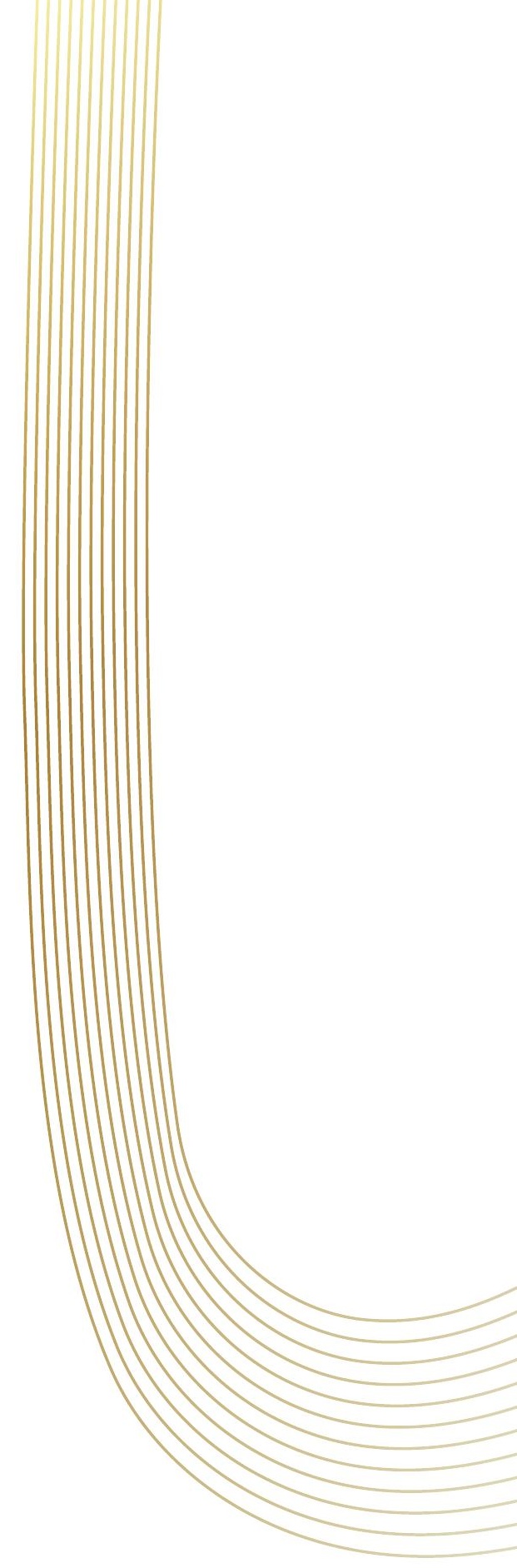


Chapter 6

Optimal timing for prediction of pathologic complete response to neoadjuvant chemoradiotherapy with diffusion-weighted MRI in patients with esophageal cancer

European Radiology 2019

A.S. Borggreve, S.E. Heethuis, M.R. Boekhoff, L. Goense, P.S.N. van Rossum, L.A.A. Brosens, A.L.H.M.W. van Lier, R. van Hillegersberg, J.J.W. Lagendijk, S. Mook, J.P. Ruurda, G.J. Meijer 


\section{Abstract}

\section{Objective}

To determine the optimal timing of diffusion-weighted magnetic resonance imaging (DWMRI) for prediction of pathologic complete response (pCR) to neoadjuvant chemoradiotherapy (nCRT) for esophageal cancer.

\section{Methods}

Patients with esophageal adenocarcinoma or squamous cell carcinoma planned to undergo nCRT followed by surgery were enrolled in this prospective study. Patients underwent 6 DW-MRI scans: 1 baseline scan before start of nCRT and weekly scans during 5 weeks of nCRT. Relative changes in mean apparent diffusion coefficient (ADC) values between the baseline scans and the scans during $\mathrm{nCRT}(\triangle \mathrm{ADC}(\%))$ were compared between pathologic complete responders ( $\mathrm{pCR}$ ) and non-pCR (tumor regression grades 2-5). The discriminative ability of $\triangle \mathrm{ADC}(\%)$ was determined based on the c-statistic.

\section{Results}

A total of 24 patients with 142 DW-MRI scans was included. pCR was observed in 7 patients (29\%). $\triangle \mathrm{ADC}(\%)$ from baseline to week 2 was significantly higher in patients with pCR versus non-pCR (median [IQR]: 36\% [30\%, 41\%] for pCR versus 16\% [14\%, 29\%] for non$\mathrm{pCR}, \mathrm{p}=0.004)$. The $\triangle \mathrm{ADC}(\%)$ of the second week in combination with histology resulted in the highest $\mathrm{c}$-statistic for the prediction of pCR versus non-pCR (0.87). The c-statistic of this model increased to 0.97 after additional exclusion of patients with a small tumor volume $(<7 \mathrm{~mL}, \mathrm{n}=3)$ and tumor histology of the resection specimen other than adenocarcinoma or squamous cell carcinoma $(n=1)$.

\section{Conclusion}

The relative change in tumor $\mathrm{ADC}(\triangle \mathrm{ADC}(\%))$ during the first 2 weeks of nCRT is the most predictive for pathologic complete response to nCRT in esophageal cancer patients. 


\section{Introduction}

Neoadjuvant chemoradiotherapy (nCRT) followed by esophagectomy is considered the standard of care for locally advanced, resectable esophageal cancer without distant metastases. ${ }^{1,2}$ Through tumor downsizing and downstaging, nCRT improves locoregional control and overall survival rates compared to surgery alone. ${ }^{2-4}$ The degree of tumor regression in response to $\mathrm{nCRT}$ is directly related to long-term survival, with pathologic complete response (pCR) resulting in the most favorable long-term prognosis. ${ }^{4,5} \mathrm{~A}$ pCR, defined as the absence of viable tumor cells at the site of the primary tumor after nCRT, is observed in around $16-29 \%$ of the patients after nCRT., ${ }^{2,6,7}$ For these pathologic complete responders, it is questioned whether they benefit from a subsequent esophagectomy, which is associated with substantial morbidity and impaired quality of life. ${ }^{8-13}$ In order to study the safety and feasibility of postponing or even omitting esophagectomy in these patients, accurate prediction of complete responders is essential.

Diffusion weighted magnetic resonance imaging (DW-MRI) is one of the modalities that is actively studied for its potential in treatment response assessment in multiple cancers, including esophageal cancer. ${ }^{14-20} \mathrm{DW}-\mathrm{MRI}$ is an appealing imaging technique because it is a quantitative method, noninvasive, relatively fast and without exposure to ionizing radiation. ${ }^{21}$ It depends on the microscopic mobility of water and is highly influenced by tissue cellularity and tissue organization. ${ }^{21}$ Treatment with chemoradiotherapy can result in the loss of cell membrane integrity and apoptosis and this process can be detected as an increase in the mean tumor apparent diffusion coefficient (ADC) ${ }^{22}$ However, a subsequent decrease in tumor ADC values may occur by fibrosis, which may complicate interpretation and predictive ability for treatment response. ${ }^{23}$ Previous studies have shown promising results for DW-MRI before nCRT, as well as during the first 2-3 weeks of nCRT in the prediction of pathologic response in esophageal cancer patients..$^{14,15,24-27}$ To investigate and further optimize the predictive ability of DW-MRI during nCRT for response assessment in esophageal cancer, this study aimed at establishing the optimal timing of DW-MRI scanning during nCRT for the prediction of $\mathrm{pCR}$ in patients with esophageal squamous cell carcinoma and adenocarcinoma.

\section{Methods}

This single center, prospective cohort study was approved by the institutional review board of the University Medical Center Utrecht (protocol ID 15-340). All participants provided written informed consent. The primary aim of the study was to research intra-fraction tumor motion and regression in order to develop patient-specific adaptive radiotherapy 
using MRI. ${ }^{28,29}$ The current analysis on the optimal timing for response prediction was a prespecified secondary aim of this prospective study, hence, not all patients who were eligible for inclusion in the prospective study were analyzed in the current analysis.

\section{Study population}

Consecutive patients with histologically confirmed squamous cell carcinoma or adenocarcinoma of the esophagus or gastroesophageal junction who were scheduled to undergo nCRT followed by esophagectomy between December 2015 and April 2018 were eligible for inclusion in the current analysis. Exclusion criteria for enrollment in the study included age $<18$ years, previous treatment with thoracic surgery or thoracic radiotherapy, and contraindications for MRI. Exclusion criteria for the current analysis included unexpected distant metastatic disease after nCRT, poor tumor visibility on DW-MRI or withdrawal from study participation. The primary diagnostic work-up consisted of an endoscopy with biopsy for diagnosis, as well as an integrated ${ }^{18} \mathrm{~F}-\mathrm{FDG}$ PET/CT scan for clinical staging.

\section{Treatment}

The neoadjuvant treatment regimen consisted of weekly intravenous administration of carboplatin and paclitaxel for 5 weeks with concurrent radiotherapy (41.4 Gy in 23 fractions of $1.8 \mathrm{~Gy}$, see Supplementary Material for details). ${ }^{2}$ Surgical resection consisted of a transthoracic or transhiatal esophagectomy with en-bloc two-field lymphadenectomy and gastric conduit reconstruction with either cervical or intrathoracic anastomosis.

\section{Histopathological assessment}

The surgical resection specimen was assessed by a specialized gastrointestinal pathologist who was blinded for the results of the DW-MRI scans. Patients were staged in accordance with the $7^{\text {th }}$ edition of the Union for International Cancer Control (UICC). ${ }^{30}$ Pathologic tumor regression was reported according to the Mandard system (tumor regression grade [TRG] 1 [pCR, ypT0]: complete response with absence of residual cancer cells, TRG 2: rare residual cancer cells scattered through fibrosis, TRG 3: increase in the number of residual cancer cells, but fibrosis still predominates, TRG 4: residual cancer outgrowing fibrosis, TRG 5: absence of regressive changes). ${ }^{31}$ In the absence of macroscopic tumor, any abnormally appearing tissue was evaluated in order to make an adequate assessment of the presence of residual tumor and the effects of therapy.

\section{Image acquisition}

Patients underwent 6 sequential MRI scans. One baseline MRI scan was performed at a median of 5 days (interquartile range [IQR]: 4-8 days) prior to nCRT in addition to the 
conventional diagnostic work-up. Subsequently, 5 additional MRI scans were performed weekly during nCRT (see Supplementary Figure 1 for the study design).

All images were acquired on a 1.5T Philips Ingenia. Respiratory-triggered transverse anatomical T2-weighted scans (tT2W) and DW-MRI scans with 3 b-values (0, 200 and 800 $\mathrm{s} / \mathrm{mm}^{2}$ ) were acquired in coronal planes (see Supplementary Methods for details).

\section{Image analysis}

The primary tumor - excluding the lumen - was delineated based on the signal of the baseline DW-MRI scans with a b-value of $800 \mathrm{~s} / \mathrm{mm}^{2}$ using open source software with a semi-automated delineation method (ITK-SNAP, www.itksnap.org), ${ }^{32,33}$ allowing for manual editing by 2 readers (A.S.B. and S.E.H.) in consensus. The primary contouring was propagated to the DW-MRI scans of the subsequent weeks, followed by manual adjustment by 1 reader (A.S.B.) based on signal reduction on the b800 DW-MRI scans and tumor regression on the tT2W scans using in-house developed delineation software. ${ }^{34}$ Contouring was performed conservatively to avoid edges of the tumor boundaries, as ADC values at the boundaries might be unreliable due to motion or image distortions. ${ }^{15}$ In all cases, the apparent tumor bed was verified based on the tT2 $\mathrm{W}$ images. Since the in-house developed delineation software interprets images as $3 \mathrm{D}$ volumes, there was no need to generate multiplanar reconstructions. The readers were blinded to patient-related characteristics and clinical outcome in terms of pathologic response.

ADC maps were generated for each slice based on a mono-exponential model fitted on b-values of 0, 200 and $800 \mathrm{~s} / \mathrm{mm}^{2}$, as based on earlier experience. ${ }^{14,15}$ Mean tumor ADC values were extracted from the DW-MRI volume of interests. The relative changes in mean $\mathrm{ADC}$ values between the baseline scans and the scans during nCRT were calculated and included in the analyses, as based on previous literature $\left(\triangle \mathrm{ADC}(\%)=\left[\right.\right.$ mean $\mathrm{ADC}_{\text {week(n) }}-$ mean $\mathrm{ADC}_{\text {baseline }}$ ] / mean $\left.\mathrm{ADC}_{\text {baseline }}\right) \cdot .^{14,15,24}$

\section{Statistical analysis}

Patient and treatment-related characteristics are described as counts with percentages, mean ( \pm standard deviation $[\mathrm{SD}]$ ) or median (IQR). The median delineated tumor volume on the baseline DW-MRI scan was compared between patients with a pCR and non-pCR using the nonparametric Mann-Whitney U test.

In order to determine the optimal timing of DW-MRI scanning for prediction of $\mathrm{pCR}$, the $\triangle \mathrm{ADC}(\%)$ was compared between patients with a pCR and non-pCR per week using the Mann-Whitney $\mathrm{U}$ test. The ability of the $\triangle \mathrm{ADC}(\%)$ parameters per week to discriminate between $\mathrm{pCR}$ and non-pCR was quantified using ridge regression, including tumor histology as determined on the tumor biopsy - an important known factor to impact pCR. Subsequently, the area under the receiver operating characteristic (ROC) curve (c-statistic) 
was calculated. Missing ADC values were imputed with multiple imputation. Subsequently, $\triangle \mathrm{ADC}(\%)$ values were calculated and the ridge regression model was fitted on all imputed datasets (see Supplementary material for details).

Furthermore, in order to determine whether the results of future studies could be optimized when applying additional exclusion criteria, a post-hoc sensitivity analysis was performed. Patients with small tumor volumes (as the signal blurring caused by respiratory motion is more pronounced in small tumors) as well as patients with a histologic tumor type other than adenocarcinoma or squamous cell carcinoma based on the resection specimen were excluded. The performance measure of interest in this sensitivity analysis was the c-statistic. All statistical analyses were performed using $\mathrm{R}$ software for statistical computing version 3.5.1 ('mice'35, 'glmnet' ${ }^{36}$ and 'Hmisc'37 packages, www.R-project.org). The significance level was set at $\mathrm{p}<0.05$. No corrections for multiple testing were performed, as the universal null hypothesis was not of interest. ${ }^{38}$ Furthermore, as this study was of descriptive nature, no formal power calculation was performed.

\section{Results}

\section{Patients}

A total of 32 patients with newly diagnosed esophageal cancer were enrolled in the prospective study. Of these patients, 8 were excluded for the current analysis based on unexpected distant metastatic disease after nCRT $(n=2)$, tumor histology other than squamous cell carcinoma or adenocarcinoma as based on the primary tumor biopsy ( $\mathrm{n}=$ $2)$, poor tumor visibility on DW-MRI $(n=3)$ or withdrawal from study participation $(n=1)$ (Supplementary Figure 2). The final study population comprised 24 patients with a mean age of 65 years ( \pm 8 years) and all but 2 were male (92\%). The majority of the patients had an adenocarcinoma (67\%). Median time between nCRT and esophagectomy was 10 weeks (IQR: 7-14 weeks). A pCR (TRG 1) after nCRT was observed in 7 patients (29\%). Table 1 gives an overview of the clinical characteristics of the study population.

All patients received 5 cycles of chemotherapy and the full course of radiation therapy. In 1 patient it was decided during nCRT to extend the regimen with 1 week, to a total dose of 50.4 Gy and 6 cycles of chemotherapy. Pre-treatment DW-MRI scans were available in all patients. Two DW-MRI scans during nCRT were missing due to patient's refusal $(n=1)$ or image acquisition problems $(n=1)$, resulting in a total of 142 MRI scans.

The median delineated tumor volume on the baseline DW-MRI scan was $15 \mathrm{~mL}$ (IQR: 11 $-23 \mathrm{~mL}$ ) and did not significantly differ between pCR and non-pCR patients (median [IQR]: $11 \mathrm{~mL}$ [7 - $22 \mathrm{~mL}$ ] versus $16 \mathrm{~mL}[11-23 \mathrm{~mL}$ ], respectively, $\mathrm{p}=0.318$ ). 
Table 1. Clinical characteristics of the study population.

\begin{tabular}{|c|c|c|c|c|c|c|}
\hline \multirow[b]{2}{*}{ Characteristic } & \multicolumn{2}{|c|}{$\begin{array}{l}\text { Full cohort } \\
\quad(n=24)\end{array}$} & \multicolumn{2}{|c|}{$\begin{array}{c}\text { pCR } \\
(\mathbf{n}=7)\end{array}$} & \multicolumn{2}{|c|}{$\begin{array}{c}\text { non-pCR } \\
(n=17)\end{array}$} \\
\hline & $\mathbf{n}$ & $(\%)$ & $\mathbf{n}$ & $(\%)$ & $\mathbf{n}$ & $(\%)$ \\
\hline Age at diagnosis, in years (mean $\pm S D$ ) & \multicolumn{2}{|c|}{$65 \pm 8$} & \multicolumn{2}{|c|}{$68 \pm 7$} & \multicolumn{2}{|c|}{$64 \pm 8$} \\
\hline \multicolumn{7}{|l|}{ Sex } \\
\hline Male & 22 & $92 \%$ & 7 & $100 \%$ & 15 & $88 \%$ \\
\hline Female & 2 & $8 \%$ & 0 & $0 \%$ & 2 & $12 \%$ \\
\hline \multicolumn{7}{|l|}{ Tumor location } \\
\hline Middle esophagus & 1 & $4 \%$ & 0 & $0 \%$ & 1 & $6 \%$ \\
\hline Distal esophagus & 22 & $92 \%$ & 7 & $100 \%$ & 15 & $88 \%$ \\
\hline Gastroesophageal junction & 1 & $4 \%$ & 0 & $0 \%$ & 1 & $6 \%$ \\
\hline \multicolumn{7}{|l|}{ Clinical T stage ${ }^{\star}$} \\
\hline cT2 & 1 & $4 \%$ & 0 & $0 \%$ & 1 & $6 \%$ \\
\hline cT3 & 23 & $96 \%$ & 7 & $100 \%$ & 16 & $94 \%$ \\
\hline \multicolumn{7}{|l|}{ Clinical N stage $^{*}$} \\
\hline cN0 & 8 & $33 \%$ & 3 & $43 \%$ & 5 & $29 \%$ \\
\hline $\mathrm{cN} 1$ & 13 & $54 \%$ & 2 & $29 \%$ & 11 & $65 \%$ \\
\hline $\mathrm{cN} 2$ & 2 & $8 \%$ & 2 & $29 \%$ & 0 & $0 \%$ \\
\hline $\mathrm{cN} 3$ & 1 & $4 \%$ & 0 & $0 \%$ & 1 & $6 \%$ \\
\hline \multicolumn{7}{|l|}{ Histologic tumor type (biopsy) } \\
\hline Adenocarcinoma & 16 & $67 \%$ & 3 & $43 \%$ & 13 & $76 \%$ \\
\hline Squamous cell carcinoma & 8 & $33 \%$ & 4 & $57 \%$ & 4 & $24 \%$ \\
\hline \multicolumn{7}{|l|}{ Tumor regression grade (TRG) } \\
\hline TRG 1 (pCR) & 7 & $29 \%$ & 7 & $100 \%$ & NA & \\
\hline TRG 2 & 6 & $25 \%$ & NA & & 6 & $35 \%$ \\
\hline TRG 3 & 7 & $29 \%$ & NA & & 7 & $41 \%$ \\
\hline TRG 4 & 3 & $13 \%$ & NA & & 3 & $18 \%$ \\
\hline TRG 5 & 1 & $4 \%$ & NA & & 1 & $6 \%$ \\
\hline \multicolumn{7}{|l|}{ Pathological T stage $^{*}$} \\
\hline урТ0 & 7 & $29 \%$ & 7 & $100 \%$ & NA & \\
\hline урТ1 & 3 & $13 \%$ & NA & & 3 & $18 \%$ \\
\hline урТ2 & 6 & $25 \%$ & NA & & 6 & $35 \%$ \\
\hline урТ3 & 8 & $33 \%$ & NA & & 8 & $47 \%$ \\
\hline \multicolumn{7}{|l|}{ Pathological N stage $e^{\star}$} \\
\hline ypNo & 15 & $63 \%$ & 6 & $86 \%$ & 9 & $53 \%$ \\
\hline ypN1 & 4 & $17 \%$ & 1 & $14 \%$ & 3 & $18 \%$ \\
\hline $\mathrm{ypN} 2$ & 4 & $17 \%$ & 0 & $0 \%$ & 4 & $24 \%$ \\
\hline ypN3 & 1 & $4 \%$ & 0 & $0 \%$ & 1 & $6 \%$ \\
\hline \multicolumn{7}{|l|}{ Surgical approach } \\
\hline Thoracolaparoscopic & 18 & $75 \%$ & 5 & $71 \%$ & 13 & $76 \%$ \\
\hline Laparoscopic transhiatal & 5 & $21 \%$ & 1 & $14 \%$ & 4 & $24 \%$ \\
\hline Open transthoracic & 1 & $4 \%$ & 1 & $14 \%$ & 0 & $0 \%$ \\
\hline Lymph node yield (median, IQR) & 30 & $(19-36)$ & 29 & $(23-40)$ & 30 & $(19-35)$ \\
\hline Positive lymph nodes harvested (median, IQR) & 0 & $(0-1)$ & 0 & $(0-0)$ & 1 & $(0-3)$ \\
\hline
\end{tabular}

$I Q R$ interquartile range; $N A$ not applicable; $p C R$ pathologic complete response; $S D$ standard deviation; TRG tumor regression grade

* Clinical and histopathologic T- and N-stage are based on UICC TNM 7th edition. 


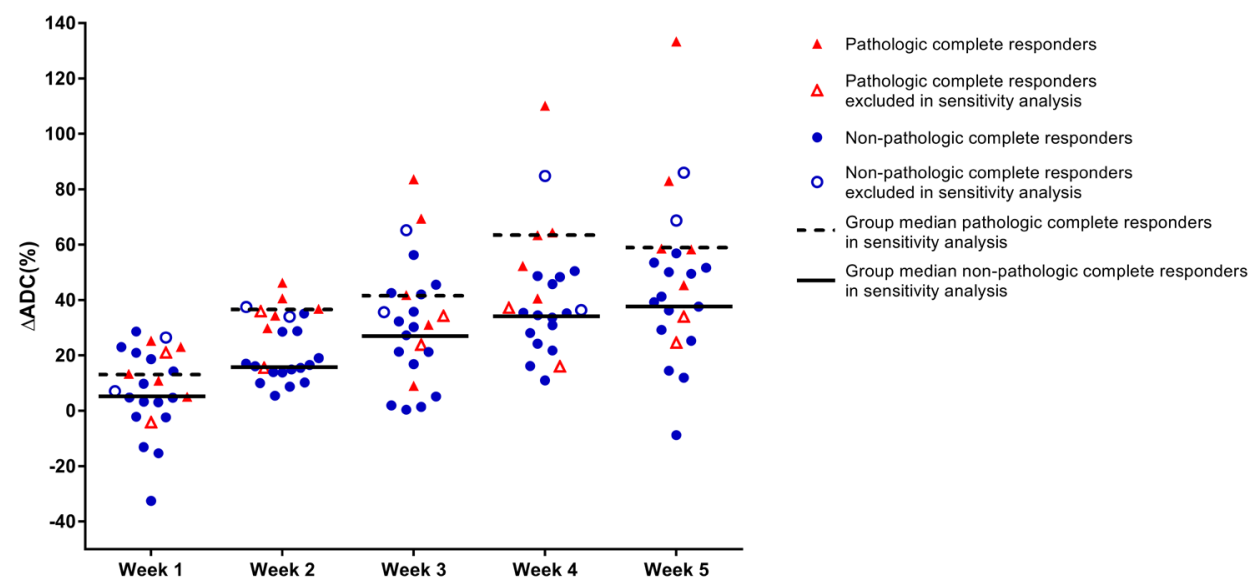

Figure 1. Relative changes in ADC values between baseline scans and scans during neoadjuvant chemoradiotherapy between pathologic complete responders (pCR, red triangles) and poor responders (non-pCR, blue circles) Patients who were excluded in the post-hoc sensitivity analysis are marked with an empty symbol.

\section{ADC changes during nCRT}

The relative increase in tumor ADC from baseline DW-MRI scans to scans acquired in the second week of $\mathrm{nCRT}\left(\triangle \mathrm{ADC}_{\text {week } 2}\right)$ was significantly associated with pCR (median [IQR]: $36 \%$ [30\% - 41\%] for pCR versus $16 \%$ [14\% - 29\%] for non-pCR, $\mathrm{p}=0.004)$. In contrast, relative changes in ADC from baseline to DW-MRI scans acquired in the other weeks of nCRT were not significantly different between pCR and non-pCR groups (Table 2, Figure 1). Figure 2 presents baseline MRI scans and MRI scans in the second week of nCRT of a patient with pCR.

ROC curve analyses after ridge penalized regression analyses taking histology into account, demonstrated the highest c-statistic of 0.87 for the relative ADC increase from baseline to week 2 of nCRT $\left(\triangle \mathrm{ADC}_{\text {week } 2}\right.$ ) combined with histology. Poorer discriminative ability was observed for histology alone (c-statistic: 0.67 ) or histology in combination with the $\triangle \mathrm{ADC}(\%)$ of the other weeks (c-statistics: $0.73-0.80$, Table 3, Figure 3). A predictive probability plot for pCR based on $\triangle \mathrm{ADC}(\%)$ from baseline to week 2 for squamous cell carcinomas and adenocarcinomas is presented in Figure 4, demonstrating an increase in the probability for $\mathrm{pCR}$ when $\triangle \mathrm{ADC}(\%)$ increases.

\section{Sensitivity analyses}

For the post-hoc sensitivity analysis, an additional 4 patients were excluded based on a delineated tumor volume on the baseline DW-MRI scan $<7 \mathrm{~mL}(\mathrm{n}=3)$ and tumor histology other than adenocarcinoma or squamous cell carcinoma as based on the resection specimen 


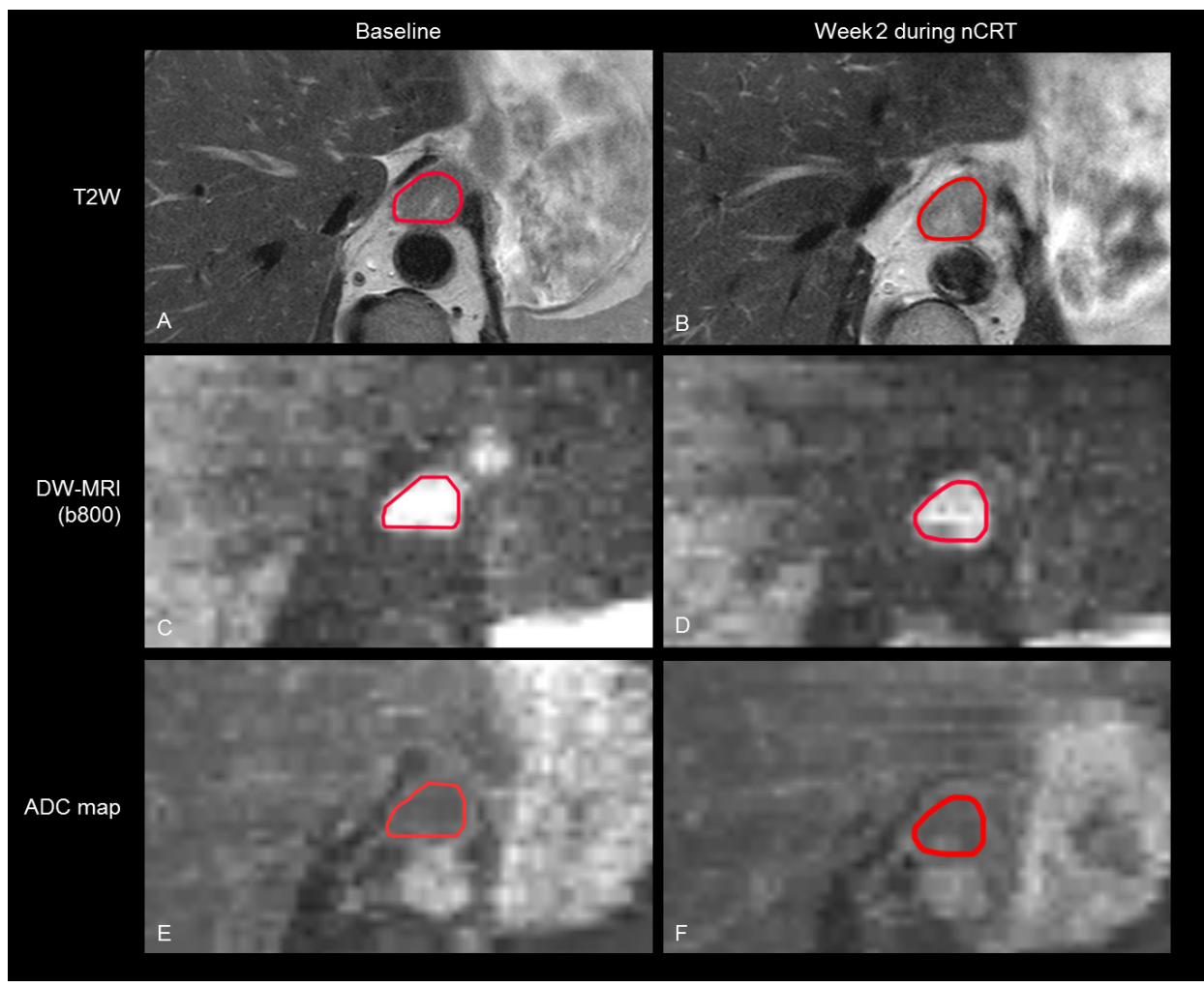

Figure 2. Patient with a cT3N2M0 distal esophageal squamous cell carcinoma with a pathologic complete response (pCR, TRG 1) to neoadjuvant chemoradiotherapy and a $\triangle \mathrm{ADC}_{\text {week2 }}$ of $29 \%$. T2 weighted images (A and B), diffusion-weighted images ( $\mathrm{b}$-value $\left.=800 \mathrm{~s} / \mathrm{mm}^{2}\right)(\mathbf{C}$ and D) and ADC maps (E and F) on a 1.5T MR scanner before $(\mathbf{A}, \mathbf{C}, \mathbf{E})$ and in the second week of neoadjuvant chemoradiotherapy $(\mathbf{B}, \mathbf{D}, \mathbf{F})$.

$(\mathrm{n}=1)$. In this study population of 20 patients, 5 patients had a pCR of which 2 adenocarcinomas and 3 squamous cell carcinomas. Exclusion of these additional 4 patients resulted in significant differences between patients with pCR and non-pCR in $\triangle A D C(\%)$ from baseline to weeks 2, 4 and 5 (Table 2). Furthermore, the c-statistics improved for the regression models with $\triangle \mathrm{ADC}(\%)$ of weeks $1,2,3,4$ and 5 to $0.84,0.97,0.77,0.93$ and 0.90 , respectively (Table 3).

Multiple imputation of the missing ADC value of week 4 and week 5 did not substantially impact the results of the regression analysis in terms of the observed c-statistics in the entire cohort ( 0.80 and 0.72 in the imputed datasets compared to 0.79 and 0.75 in the complete case datasets, respectively) or in the sensitivity analysis ( 0.93 and 0.87 in the imputed dataset compared to 0.93 and 0.90 in the complete case dataset, respectively) (Supplementary Table 2). 
Table 2. Relative changes in ADC per week during neoadjuvant chemoradiotherapy between esophageal cancer patients with a pathologic complete response and non-pathologic complete response.

\begin{tabular}{|c|c|c|c|c|}
\hline & & \multicolumn{2}{|c|}{ Median $\triangle A D C(\%)(I Q R)$} & \multirow[b]{2}{*}{ p-value ${ }^{*}$} \\
\hline & & pCR & non-pCR & \\
\hline \multirow[t]{5}{*}{ Full cohort $(n=24)$} & Week 1 & $13(5,23)$ & $5(-2,19)$ & 0.260 \\
\hline & Week 2 & $36(30,41)$ & $16(14,29)$ & 0.004 \\
\hline & Week 3 & $34(24,69)$ & $30(17,42)$ & 0.318 \\
\hline & Week 4 & $52(37,64)$ & $35(26,47)$ & 0.065 \\
\hline & Week 5 & $58(34,83)$ & $40(27,53)$ & 0.198 \\
\hline \multirow{5}{*}{$\begin{array}{l}\text { Sensitivity analyses } \\
(n=20)^{¥}\end{array}$} & Week 1 & $13(11,23)$ & $5(-2,19)$ & 0.098 \\
\hline & Week 2 & $37(24,41)$ & $16(10,19)$ & 0.001 \\
\hline & Week 3 & $42(31,69)$ & $27(5,42)$ & 0.168 \\
\hline & Week 4 & $63(52,64)$ & $34(24,46)$ & 0.002 \\
\hline & Week 5 & $59(58,83)$ & $38(25,50)$ & 0.003 \\
\hline
\end{tabular}

$A D C$ apparent diffusion coefficient; $I Q R$ interquartile range; $p C R$ pathologic complete response

* p-value based on Mann-Whitney U test.

$¥$ After post-hoc exclusion of additional 4 patients based on baseline tumor volume delineated on DW-MRI $<7$ $\mathrm{mL}(\mathrm{n}=3)$ and tumor histology other than adenocarcinoma or squamous cell carcinoma as based on the resection specimen $(n=1)$.

\section{Discussion}

This prospective study was designed to assess the optimal timing of DW-MRI scanning during $\mathrm{nCRT}$ for prediction of $\mathrm{pCR}$ in esophageal cancer patients. The relative change in $\mathrm{ADC}(\triangle \mathrm{ADC})$ during the first 2 weeks of nCRT demonstrated the highest predictive ability for $\mathrm{pCR}$ at the time of surgery. This is of important clinical value as early response evaluation could enable individualized treatment regimens. Accurate assessment of response to nCRT is not only important for safe implementation of an organ-sparing approach in patients with pCR, but also to adapt treatment strategies in expected poor responders. Improving the accuracy of response evaluation after nCRT may provide improved outcomes for both patient groups.

In order to improve patient-friendliness of disease monitoring and (re)staging procedures, it is important to minimize the burden of these procedures. As demonstrated before, DWMRI is generally well-tolerated by patients, although shorter acquisition times as well as altered body positioning could further improve patient experience.$^{39}$ Our results could further aid the minimization of the burden of repeated scanning for patients, as well as optimal usage of the available (financial) resources, while assuring the best predictive ability of DW-MRI.

Previous studies focusing on DW-MRI scanning in response prediction for esophageal cancer have mostly demonstrated promising findings regarding the predictive value of 

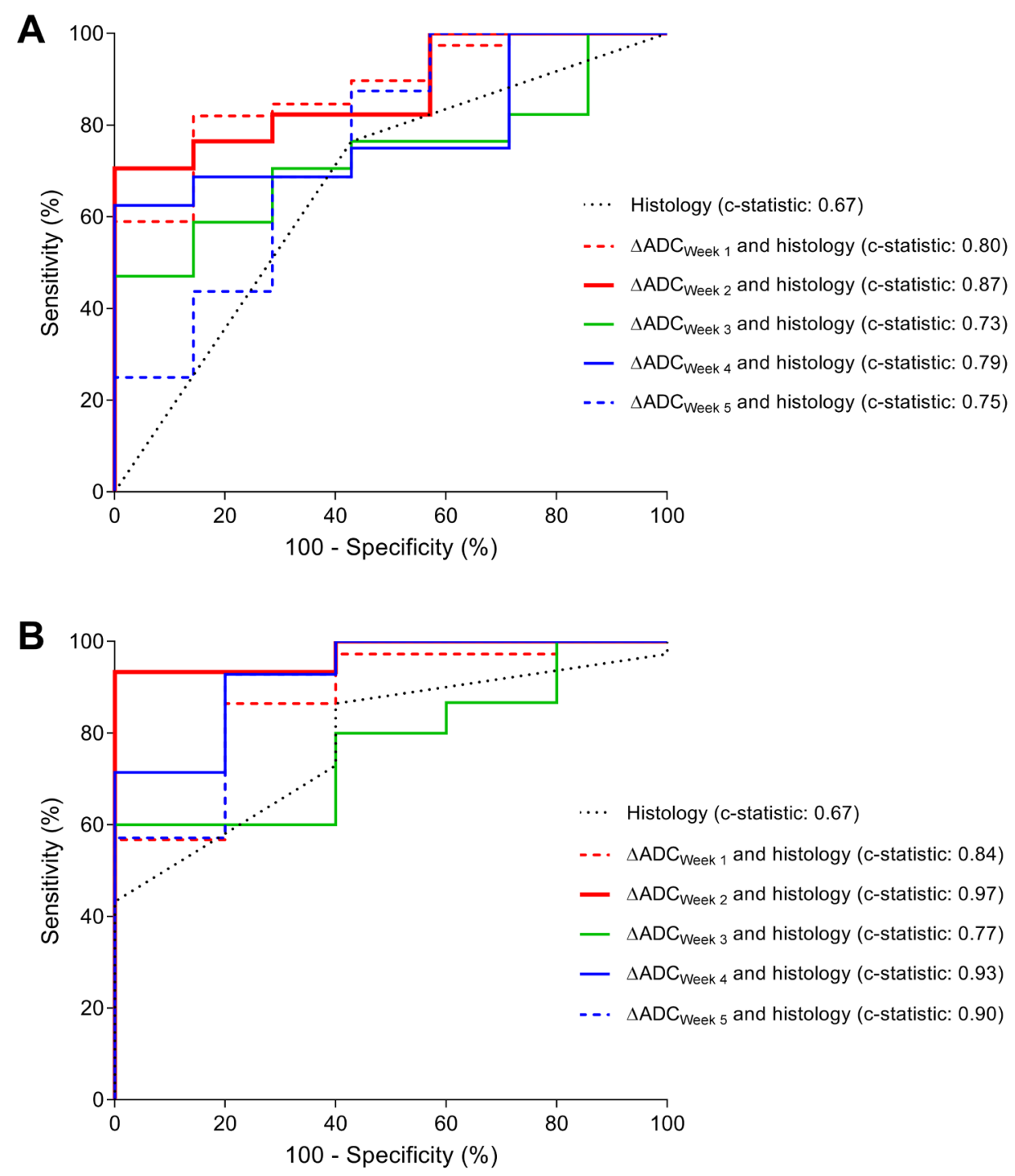

Figure 3. Receiver-operating-characteristic curve analysis for the regression models with relative changes in ADC per week, as well as histopathological tumor type, for discriminating between pCR and non-pCR patients in the full cohort (A) as well as in the sensitivity analysis (B).

$\triangle \mathrm{ADC}(\%)$ for response prediction to $\mathrm{nCRT} .14,15,18,40,41$ The majority of these studies reported significant differences between responders and non-responders in $\triangle \mathrm{ADC}(\%)$ from baseline to during $\mathrm{nCRT},{ }^{14,15,24,41}$ whereas others report significant differences in $\triangle \mathrm{ADC}(\%)$ from baseline to follow-up after nCRT. ${ }^{18,41}$ Nevertheless, not all studies found the same predictive 
Table 3. Ridge regression analyses demonstrating the discriminatory value of DW-MRI parameters per week with pathologic complete response (TRG 1) as outcome variable.

\begin{tabular}{|c|c|c|c|c|c|c|}
\hline \multirow[t]{2}{*}{ Intercept and predictors } & \multicolumn{3}{|c|}{ Full cohort $(n=24)$} & \multicolumn{3}{|c|}{ Sensitivity analyses $(n=20)^{*}$} \\
\hline & $\boldsymbol{\beta}$ & OR & c-statistic & $\beta$ & OR & c-statistic \\
\hline \multicolumn{7}{|c|}{ Histology } \\
\hline Intercept & -1.00 & & 0.67 & -1.28 & & 0.67 \\
\hline Squamous cell carcinoma ${ }^{¥}$ & 0.33 & 1.39 & & 0.49 & 1.63 & \\
\hline \multicolumn{7}{|c|}{ Week 1} \\
\hline Intercept & -1.74 & & 0.80 & -2.30 & & 0.84 \\
\hline$\Delta \mathrm{ADC}_{\text {week } 1}(\%)$ & 0.04 & 1.04 & & 0.06 & 1.06 & \\
\hline Squamous cell carcinoma ${ }^{¥}$ & 1.29 & 3.63 & & 1.44 & 4.24 & \\
\hline \multicolumn{7}{|c|}{ Week 2} \\
\hline Intercept & -3.45 & & 0.87 & -5.17 & & 0.97 \\
\hline$\Delta \mathrm{ADC}_{\text {week } 2}(\%)$ & 0.09 & 1.09 & & 0.15 & 1.16 & \\
\hline Squamous cell carcinoma ${ }^{¥}$ & 0.89 & 2.44 & & 0.32 & 1.37 & \\
\hline \multicolumn{7}{|c|}{ Week 3} \\
\hline Intercept & -1.09 & & 0.73 & -1.76 & & 0.77 \\
\hline$\Delta \mathrm{ADC}_{\text {week3 }}(\%)$ & 0.00 & 1.00 & & 0.02 & 1.02 & \\
\hline Squamous cell carcinoma ${ }^{¥}$ & 0.20 & 1.23 & & 0.44 & 1.56 & \\
\hline \multicolumn{7}{|c|}{ Week 4} \\
\hline Intercept & -1.61 & & 0.79 & -4.76 & & 0.93 \\
\hline$\Delta \mathrm{ADC}_{\text {week } 4}(\%)$ & 0.01 & 1.01 & & 0.08 & 1.08 & \\
\hline Squamous cell carcinoma ${ }^{¥}$ & 0.50 & 1.65 & & 0.16 & 1.18 & \\
\hline \multicolumn{7}{|c|}{ Week 5} \\
\hline Intercept & -1.57 & & 0.75 & -4.03 & & 0.90 \\
\hline$\Delta \mathrm{ADC}_{\text {week } 5}(\%)$ & 0.01 & 1.01 & & 0.06 & 1.06 & \\
\hline Squamous cell carcinoma ${ }^{*}$ & 0.69 & 1.99 & & 0.64 & 1.90 & \\
\hline
\end{tabular}

$A D C$ apparent diffusion coefficient; $c$-statistic concordance statistic; OR odds ratio; $p C R$ pathologic complete response

Note. Due to rounding, the reported odds ratios might not precisely correspond with the reported beta regression coefficients.

* After post-hoc exclusion of additional 4 patients based on baseline tumor volume delineated on DW-MRI $<7$ $\mathrm{mL}(\mathrm{n}=3)$ and tumor histology other than adenocarcinoma or squamous cell carcinoma as based on the resection specimen $(n=1)$.

$¥$ Adenocarcinoma was used as reference category.

effect size, nor did they all report a significant relation between $\triangle \mathrm{ADC}(\%)$ and response. ${ }^{42,43}$ Several factors may account for these differences. First, ADCs were calculated based on different b-values varying from 0 and $1000^{41}, 0$ and $600^{24}$ to 0,200 and $800 .^{14,15}$ The choice of b-values, number of signal averages (NSA's) per b-value, as well as the calculation of ADC values by various modeling strategies, are known to impact tumor ADC estimates. ${ }^{21}$ Second, delineation methods for determining the volume of interest differ. Some studies consider the entire tumor volume as volume of interest, ${ }^{14,15,18}$ whereas others only delineated the tumor on the most representative tumor slice. ${ }^{41}$ Lastly, the nCRT regimen of choice varied between the studies. 


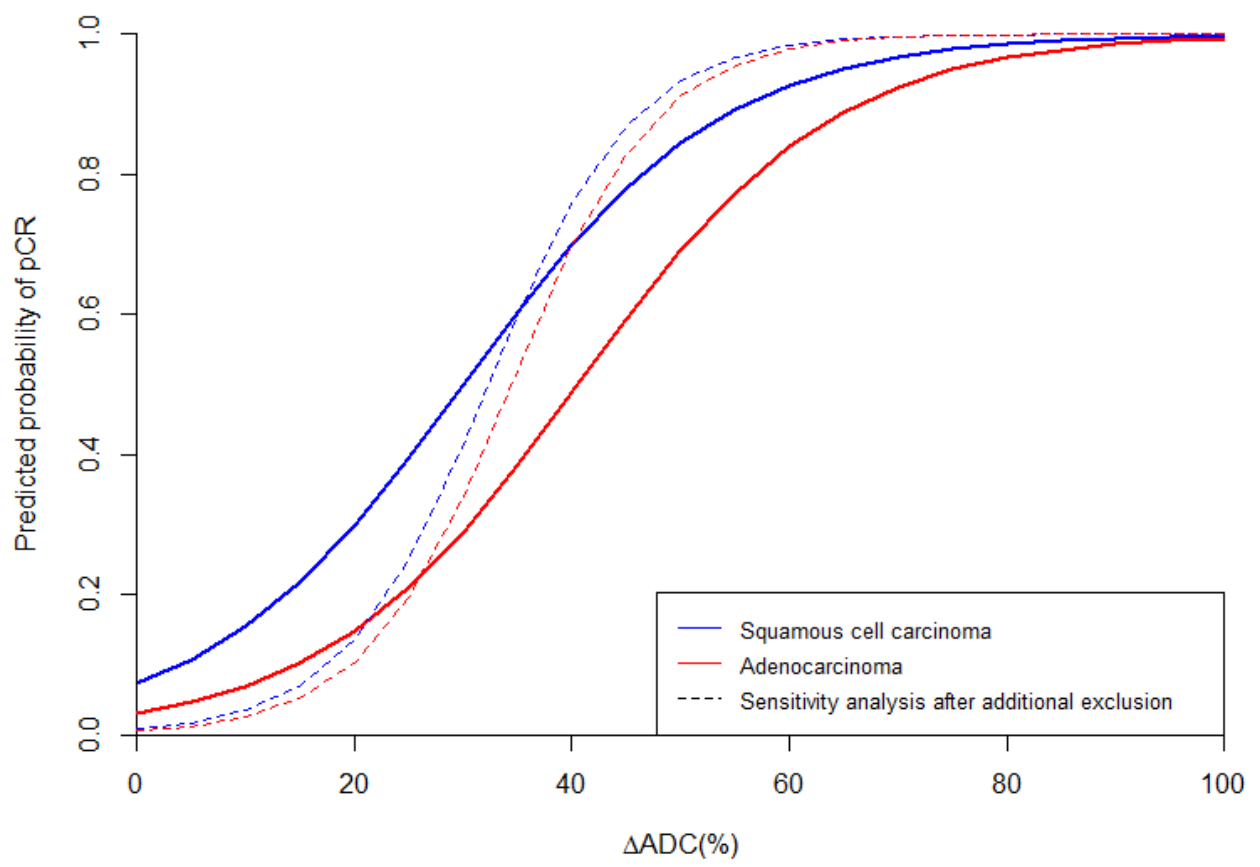

Figure 4. Predictive probability plot for pathologic complete response (pCR) based on the fitted regression model including relative changes in $\mathrm{ADC}(\triangle \mathrm{ADC}(\%))$ from baseline to week 2 for squamous cell carcinomas (blue) and adenocarcinomas (red). The dashed lines represent the probability plot for the sensitivity analysis after additional exclusion of 4 patients.

Similar to our study, Wang et al. ${ }^{24}$ performed weekly DW-MRI scanning during CRT in esophageal cancer patients to determine the optimal timing of response evaluation with DW-MRI. They also demonstrated DW-MRI scanning in the second or third week of CRT to be optimal for response assessment. As their study included only esophageal squamous cell carcinomas, used imaging response criteria as a reference standard (i.e. RECIST criteria $^{44}$ that assessed $52 \%$ of the patients to be a complete responder) instead of histopathology, used a different nCRT regimen (chemotherapy consisting of cisplatin with either 5-fluorouracil or paclitaxel, and radiotherapy consisting of $60 \mathrm{~Gy}$ in 30 fractions) and did not use $\triangle \mathrm{ADC}(\%)$ but only single time point $\mathrm{ADC}$ values as measured, similar findings might not have been expected. Additionally, earlier studies by our group as well as the University of Texas MD Anderson Cancer Center that performed DW-MRI in the second or third week found these $\triangle \mathrm{ADC}(\%)$ values to be highly predictive of response to nCRT. ${ }^{14,15}$ Together, this supports the robustness of the findings from the current study. The interobserver reproducibility of tumor delineation on DW-MRI and ADC measurements in esophageal cancer was shown to be very good by two previous studies, especially for the 
semi-automated volumetric measurement method (intraclass correlation coefficient: 0.96, 95\% CI: 0.91-0.98, p<0.001), which was also applied in the current study. ${ }^{15,43}$ One of these studies compared manual delineation of a region of interest on the most representative tumor slice to semi-automatic delineation of the whole tumor volume, and found negligible differences in mean ADC measurements (between - $0.25 \%$ and $0.31 \%) .{ }^{15}$ A voxel-based analytical method, where changes in individual voxels can be monitored, may even provide more reliable results. ${ }^{43}$ However, such an approach is challenging, since tumor regression is observed during nCRT and the esophagus is a moving organ ${ }^{28}$, making spatial registration of DW-MRI obtained before and after start of nCRT complicated.

To improve external validity of our results, no cut-off values for ADC or $\triangle \mathrm{ADC}$ measurements for classification of complete responders versus non responders are reported in our study. Cut-off values are likely to be highly influenced by delineation techniques and determination of a $2 \mathrm{D}$ or $3 \mathrm{D}$ region of interest, as well as the b-values on which ADCs are calculated. To demonstrate this, we highlighted previously reported significant cut-off values for $\triangle \mathrm{ADC}(\%), \mathrm{ADC}_{\text {mean }}$ and $\mathrm{ADC}_{\text {median }}$ for discrimination of responders versus nonresponders in the data of the current study in Supplementary Figure 3.

A recent meta-analysis demonstrated a pooled AUC of 0.91 (95\% CI: 0.89-0.94) of $\triangle \mathrm{ADC}(\%)$ values for treatment response prediction in esophageal cancer based on 4 studies. ${ }^{40}$ We were not able to reproduce these results in our full cohort, but this cohort included 3 patients with small tumor volumes $(<7 \mathrm{~mL})$ and 1 patient who had a mixed adenoneuroendocrine carcinoma (MANEC) upon histopathological evaluation of the resection specimen. As MANECs are known to respond well to nCRT during treatment (which is reflected by an increase in ADC in the first weeks) but progress rapidly after completion, the inclusion of this patient clearly influenced the obtained results in this relatively small cohort. ${ }^{45,46}$ Exclusion of the aforementioned patients dramatically improved the performance of $\triangle \mathrm{ADC}(\%)$ for $\mathrm{pCR}$ prediction, resulting in a c-statistic of 0.97 for week 2. This also demonstrated that the predictive value of relative changes in ADC seems decreased for small tumors, which might be explained by the respiratory movement of the tumor during the DW-MRI scan. Typically, respiratory motion amplitudes of $1-2 \mathrm{~cm}$ are observed during scanning, ${ }^{28,47}$ which might negatively impact the quantitative ADC assessment especially in small tumors. Improvement in pCR prediction in future studies may be obtained by incorporating motion management techniques.

Previous work has also demonstrated additional value of DCE-MRI, as well as PET-CT scanning in the prediction of treatment response in esophageal cancer patients. ${ }^{15,48-52}$ Furthermore, emerging biomarkers such as circulating tumor DNA might further improve the predictive performance and might contribute to the safe investigation of an organsparing approach for predicted pCR to nCRT.

Significant strengths of the current study include the consistent use of one nCRT regimen 
for all patients, the presence of a histopathologic reference standard in all patients, the inclusion of both squamous cell carcinomas and adenocarcinomas, as well as the consistent delineation by semi-automatic contouring. However, the specific hardware characteristics of the MR scanner and scan sequences, as well as the applied delineation technique and calculation of the ADC map by a mono-exponential model based on 3 b-values may limit the generalizability of the results. In addition, whole-tumor summary statistics (such as the mean ADC) are easily applicable, but fail to fully address the important issue of tumor heterogeneity. ${ }^{21}$ Furthermore, the relatively small study population might have led to falsenegative results (type II error) for differences in $\triangle \mathrm{ADC}(\%)$ between responders and nonresponders from baseline to the other weeks than week 2. Moreover, patients were included during a rather long study period of 2.5 years, since many eligible patients refused participation in this demanding and time-consuming study with weekly MRI scanning. However, the patients generally tolerated the MRI scans well (only 3 patients cancelled 1 MRI scan during their treatment in the entire cohort of 32 patients) and no adverse events occurred. Lastly, DW-MRI scanning is currently not routinely used in the staging of patients with esophageal cancer, which challenges the direct implementation of the results in clinical practice.

Future comparative studies should focus on further improving response evaluation after nCRT. In this regard, the recently started Dutch multicenter PRIDE study will further investigate the findings of the DW-MRI pilot studies in a larger patient cohort and aims at developing a multimodal clinically applicable prediction model..$^{53}$

In conclusion, treatment-induced change in tumor $\mathrm{ADC}$ as measured on DW-MRI during the second week is most predictive for pCR to nCRT in esophageal squamous cell carcinoma and adenocarcinoma. 


\section{References}

1. Lordick F, Mariette C, Haustermans K, et al. Oesophageal cancer: ESMO Clinical Practice Guidelines for diagnosis, treatment and follow-up. Ann Oncol. 2016;27:v50-v57.

2. van Hagen P, Hulshof MCCM, van Lanschot JJB, et al. Preoperative Chemoradiotherapy for Esophageal or Junctional Cancer. N Engl J Med. 2012;366:2074-2084.

3. Sjoquist KM, Burmeister BH, Smithers BM, et al. Survival after neoadjuvant chemotherapy or chemoradiotherapy for resectable oesophageal carcinoma: an updated meta-analysis. Lancet Oncol. 2011;12:681-92.

4. Shapiro J, Lanschot JJB Van, Hulshof MCCM, et al. Neoadjuvant chemoradiotherapy plus surgery versus surgery alone for oesophageal or junctional cancer (CROSS): long-term results of a randomised controlled trial. Lancet Oncol. 2015;16:1090-1098.

5. Steffen T, Dietrich D, Schnider A, et al. Recurrence Patterns and Long-Term Results After Induction Chemotherapy, Chemoradiotherapy, and Curative Surgery in Patients With Locally Advanced Esophageal Cancer. Ann Surg. 2019;269:83-87.

6. Walsh TN, Noonan N, Hollywood D, et al. A Comparison of Multimodal Therapy and Surgery for Esophageal Adenocarcinoma. N Engl J Med. 1996;335:462-467.

7. Burmeister BH, Smithers BM, Gebski V, et al. Surgery alone versus chemoradiotherapy followed by surgery for resectable cancer of the oesophagus: a randomised controlled phase III trial. Lancet Oncol. 2005;6:659_ 668.

8. Djärv T, Lagergren J, Blazeby JM, Lagergren P. Long-term health-related quality of life following surgery for oesophageal cancer. Br J Surg. 2008;95:1121-1126.

9. Mc Cormack O, Zaborowski A, King S, et al. New-onset Atrial Fibrillation Post-surgery for Esophageal and Junctional Cancer Incidence, Management, and Impact on Short-and Long-term Outcomes. Ann Surg. 2014;260:772-778.

10. Kassis ES, Kosinski AS, Ross P, et al. Predictors of anastomotic leak after esophagectomy: An analysis of the society of thoracic surgeons general thoracic database. Ann Thorac Surg. 2013;96:1919-1926.

11. Busweiler LAD, Wijnhoven BPL, van Berge Henegouwen MI, et al. Early outcomes from the Dutch Upper Gastrointestinal Cancer Audit. Br J Surg. 2016;103:1855-1863.

12. Schandl A, Lagergren J, Johar A, Lagergren P. Health-related quality of life 10 years after oesophageal cancer surgery. Eur J Cancer. 2016;69:43-50.

13. Noordman BJ, Wijnhoven BPL, Lagarde SM, et al. Active surveillance in clinically complete responders after neoadjuvant chemoradiotherapy for esophageal or junctional cancer. Dis Esophagus. 2017;30:1-8.

14. van Rossum PS, van Lier AL, van Vulpen M, et al. Diffusion-weighted magnetic resonance imaging for the prediction of pathologic response to neoadjuvant chemoradiotherapy in esophageal cancer. Radiother Oncol. 2015;115:163-170.

15. Fang P, Musall BC, Son JB, et al. Multimodal Imaging of Pathologic Response to Chemoradiation in Esophageal Cancer. Int J Radiat Oncol Biol Phys. 2018;102:996-1001.

16. Jacobs L, Intven M, van Lelyveld N, et al. Diffusion-weighted MRI for Early Prediction of Treatment Response on Preoperative Chemoradiotherapy for Patients With Locally Advanced Rectal Cancer. Ann Surg. 2016;263:522-528.

17. Lambrecht M, Vandecaveye V, De Keyzer F, et al. Value of Diffusion-Weighted Magnetic Resonance Imaging for Prediction and Early Assessment of Response to Neoadjuvant Radiochemotherapy in Rectal Cancer: Preliminary Results. Int J Radiat Oncol. 2012;82:863-870.

18. De Cobelli F, Giganti F, Orsenigo E, et al. Apparent diffusion coefficient modifications in assessing gastrooesophageal cancer response to neoadjuvant treatment: Comparison with tumour regression grade at histology. Eur Radiol. 2013;23:2165-2174.

19. Harry VN. Novel imaging techniques as response biomarkers in cervical cancer. Gynecol Oncol. 2010;116:253-261.

20. Partridge SC, Zhang Z, Newitt DC, et al. Diffusion-weighted MRI Findings Predict Pathologic Response in Neoadjuvant Treatment of Breast Cancer: The ACRIN 6698 Multicenter Trial. Radiology. 2018; 289:618-627

21. Padhani AR, Liu G, Koh DM, et al. Diffusion-weighted magnetic resonance imaging as a cancer biomarker: consensus and recommendations. Neoplasia. 2009;11:102-25 
22. Thoeny HC, Ross BD. Predicting and monitoring cancer treatment response with diffusion-weighted MRI. J Magn Reson Imaging. 2010;32:2-16.

23. Galbán CJ, Hoff BA, Chenevert TL, Ross BD. Diffusion MRI in early cancer therapeutic response assessment. NMR Biomed. 2017;30.

24. Wang L, Liu L, Han C, et al. The diffusion-weighted magnetic resonance imaging (DWI) predicts the early response of esophageal squamous cell carcinoma to concurrent chemoradiotherapy. Radiother Oncol. 2016;121:246-251.

25. Wang L, Han C, Zhu S, et al. Investigation of using diffusion-weighted magnetic resonance imaging to evaluate the therapeutic effect of esophageal carcinoma treatment. Oncol Res Treat. 2014;37:112-6.

26. Giganti F, Salerno A, Ambrosi A, et al. Prognostic utility of diffusion-weighted MRI in oesophageal cancer: is apparent diffusion coefficient a potential marker of tumour aggressiveness? Radiol Med. 2016;121:17380 .

27. Aoyagi T, Shuto K, Okazumi S, et al. Apparent diffusion coefficient values measured by diffusion-weighted imaging predict chemoradiotherapeutic effect for advanced esophageal cancer. Dig Surg. 2011;28:252-7.

28. Heethuis SE, Borggreve AS, Goense L, et al. Quantification of variations in intra-fraction motion of esophageal tumors over the course of neoadjuvant chemoradiotherapy based on cine-MRI. Phys Med Biol. 2018;63:145019

29. Boekhoff M, Kotte A, Mook S, et al. PO-0951: What CTV-to-PTV margins are required for esophageal cancer radiotherapy? Radiother Oncol. 2018;127:S519-S520.

30. Sobin L, Gospodarowicz MWC. TNM Classification of Malignant Tumours (7th edition). Wiley-Liss, New York. 2009.

31. Mandard AM, Dalibard F, Mandard JC, et al. Pathologic assessment of tumor regression after preoperative chemoradiotherapy of esophageal carcinoma. Clinicopathologic correlations. Cancer. 1994;73:2680-6

32. Musall B. Quantitative DWI as an Early Imaging Biomarker of the Response to Chemoradiation in Esophageal Cancer. UT GSBS Dissertations and Theses (Open Access). 2017. Available via: https:// digitalcommons.library.tmc.edu/utgsbs_dissertations/805.

33. Yushkevich PA, Piven J, Hazlett HC, et al. User-guided 3D active contour segmentation of anatomical structures: Significantly improved efficiency and reliability. Neuroimage. 2006;31:1116-1128.

34. Bol GH, Kotte ANTJ, van der Heide UA, Lagendijk JJW. Simultaneous multi-modality ROI delineation in clinical practice. Comput Methods Programs Biomed. 2009;96:133-140.

35. Van Buuren S, Groothuis-Oudshoorn K. Multivariate Imputation by Chained Equations. J Stat Softw. 2011;45:1-67.

36. Friedman J, Hastie T, Tibshirani R. Regularization Paths for Generalized Linear Models via Coordinate Descent. J Stat Softw. 2010;33:1-22

37. Frank E Harrell Jr with contributions from Charles Dupont and many others. Hmisc: Harrell Miscellaneous. R package version 4.1-1. 2018.

38. Perneger TV. What's wrong with Bonferroni adjustments. BMJ. 1998;316:1236-8.

39. Goense L, Borggreve AS, Heethuis SE, et al. Patient perspectives on repeated MRI and PET/CT examinations during neoadjuvant treatment of oesophageal cancer. Br J Radiol. 2018;91:20170710.

40. Cheng B, Yu J. Predictive value of diffusion-weighted MR imaging in early response to chemoradiotherapy of esophageal cancer: a meta-analysis. Dis Esophagus. 2019;32(4).

41. Imanishi S, Shuto K, Aoyagi T, et al. Diffusion-weighted magnetic resonance imaging for predicting and detecting the early response to chemoradiotherapy of advanced esophageal squamous cell carcinoma. Dig Surg. 2013;30:240-8.

42. Li Q-W, Qiu B, Wang B, et al. Prediction of pathologic responders to neoadjuvant chemoradiotherapy by diffusion-weighted magnetic resonance imaging in locally advanced esophageal squamous cell carcinoma: a prospective study. Dis Esophagus. 2018;31(2).

43. Kwee RM, Dik AK, Sosef MN, et al. Interobserver Reproducibility of Diffusion-Weighted MRI in Monitoring Tumor Response to Neoadjuvant Therapy in Esophageal Cancer. PLoS One. 2014;9:e92211.

44. Eisenhauer EA, Therasse P, Bogaerts J, et al. New response evaluation criteria in solid tumours: Revised RECIST guideline (version 1.1). Eur J Cancer. 2009;45:228-247.

45. Ilett E, Langer S, Olsen I, et al. Neuroendocrine Carcinomas of the Gastroenteropancreatic System: A Comprehensive Review. Diagnostics. 2015;5:119-176. 
46. van der Veen A, Seesing MFJ, Wijnhoven BPL, et al. Management of resectable esophageal and gastric (mixed adeno)neuroendocrine carcinoma: A nationwide cohort study. Eur J Surg Oncol. 2018;44:19551962.

47. Lever FM, Lips IM, Crijns SPM, et al. Quantification of Esophageal Tumor Motion on Cine-Magnetic Resonance Imaging. Int J Radiat Oncol Biol Phys. 2014;88:419-424.

48. Heethuis SE, van Rossum PSN, Lips IM, et al. Dynamic contrast-enhanced MRI for treatment response assessment in patients with oesophageal cancer receiving neoadjuvant chemoradiotherapy. Radiother Oncol. 2016;120:128-135.

49. Goense L, Heethuis SE, van Rossum PSN, et al. Correlation between functional imaging markers derived from diffusion-weighted MRI and 18F-FDG PET/CT in esophageal cancer. Nucl Med Commun. 2018;39:60-67.

50. Heethuis SE, Goense L, van Rossum PSN, et al. DW-MRI and DCE-MRI are of complementary value in predicting pathologic response to neoadjuvant chemoradiotherapy for esophageal cancer. Acta Oncol. 2018;57:1201-1208.

51. Findlay JM, Dickson E, Fiorani C, et al. Temporal validation of metabolic nodal response of esophageal cancer to neoadjuvant chemotherapy as an independent predictor of unresectable disease, survival, and recurrence. Eur Radiol. 2019;29:6717-6727.

52. Jin X, Zheng X, Chen D, et al. Prediction of response after chemoradiation for esophageal cancer using a combination of dosimetry and CT radiomics. Eur Radiol. 2019;29:6080-6088

53. Borggreve AS, Mook S, Verheij M, et al. Preoperative image-guided identification of response to neoadjuvant chemoradiotherapy in esophageal cancer (PRIDE): a multicenter observational study. BMC Cancer. 2018;18:1006. 


\section{SUPPLEMENTARY MATERIAL}

\section{Methods}

\section{Radiotherapy}

All patients were treated with volumetric modulated arc radiotherapy. The gross target volume (GTV) was contoured based on information from the endoscopy and ${ }^{18} \mathrm{~F}-\mathrm{FDG}$ PET/CT scan. The extension of the GTV to the clinical target volume (CTV) was typically $3 \mathrm{~cm}$ cranially and caudally, and $0.5 \mathrm{~cm}$ circumferential without violation of anatomic boundaries. The planning target volume (PTV) expansion was $1 \mathrm{~cm}$ isotropically.

\section{Image acquisition details}

All images were acquired on a 1.5T Philips Ingenia (Best, the Netherlands), using anterior/ posterior (28-channel) receive coils. Patients were positioned in supine position with both arms next to the body. Respiratory-triggered transverse anatomical T2-weighted scans (tT2W) were acquired with a multi-slice turbo spin echo sequence $(\mathrm{TR} / \mathrm{TE}=1604 / 100 \mathrm{~ms}$, resolution $\left.=0.67 \times 0.67 \times 6.48 \mathrm{~mm}^{3}\right)$. DW-MRI scans with $3 \mathrm{~b}$-values $\left(0,200\right.$ and $\left.800 \mathrm{~s} / \mathrm{mm}^{2}\right)$ were acquired in coronal planes (number of squared averages [NSA] 4, 3 and 4 for $b=0$, 200 and $800 \mathrm{~s} / \mathrm{mm}^{2}$, respectively), using SPIR fat suppression and EPI read-out (resolution $=3.25 \times 3.25 \times 4 \mathrm{~mm}^{3}$, bandwidth per pixel $=22.9 \mathrm{~Hz}$ ) (see Supplementary Table 1). Images were obtained in coronal planes to mitigate the effects of respiratory motion on image quality, as it was demonstrated before that movement of the esophagus primarily occurs in the cranio-caudal direction. ${ }^{1}$ As such, no out-of-plane motion will occur between the diffusion preparation and the image read-out.

\section{Statistical analyses}

Ridge regression is a penalized regression method, enabling reduction of model overfitting in a situation with few events per variable. ${ }^{2}$ The applied overall penalty $\left(\lambda_{\min }\right)$ represents the minimum mean cross-validated error, which was obtained using 10 -fold cross validation. Missing ADC values were imputed with using multiple imputation (multivariate imputation by chained equations, 20 imputed datasets with a maximum number of 20 iterations for each imputation). ${ }^{3,4}$ All variables in Table 1 as well as the ADC statistics (mean, SD) of all weeks were used as predictor variables for the imputation. Subsequently, $\triangle \mathrm{ADC}(\%)$ values were calculated and the ridge regression model was fitted on all imputed datasets. Model coefficients and the c-statistic were pooled using Rubin's rules. ${ }^{5}$ 
Supplementary Table 1. Image acquisition details.

\begin{tabular}{|c|c|c|}
\hline \multicolumn{3}{|c|}{ General scanner characteristics } \\
\hline \multicolumn{2}{|c|}{ Magnetic field strength } & $1.5 \mathrm{~T}$ \\
\hline \multicolumn{2}{|l|}{ Vendor } & Philips \\
\hline \multicolumn{2}{|l|}{ Type } & Ingenia \\
\hline \multicolumn{3}{|c|}{ Protocol } \\
\hline \multirow[t]{8}{*}{$\mathrm{tT} 2 \mathrm{~W}$} & Scan plane & transverse \\
\hline & Scan mode & Multi-Slice \\
\hline & Repetition time (ms) & 1604 \\
\hline & Echo time (ms) & 100 \\
\hline & Voxel size $(\mathrm{mm})$ & 0.67 \\
\hline & Slice thickness (mm) & 6.48 \\
\hline & Flip angle & 90 \\
\hline & Navigator & Yes \\
\hline \multirow[t]{11}{*}{ DWI } & Scan plane & Coronal \\
\hline & Scan mode & Multi-Slice \\
\hline & Repetition time (ms) & 4299 \\
\hline & Echo time (ms) & 70 \\
\hline & Voxel size $(\mathrm{mm})$ & 3.25 \\
\hline & Slice thickness (mm) & 4.0 \\
\hline & Flip angle & 90 \\
\hline & b-values & $0 / 200 / 800$ \\
\hline & Number of squared averages & $4 / 3 / 4$ \\
\hline & Fat suppresion & SPIR \\
\hline & Navigator & No \\
\hline
\end{tabular}

Supplementary Table 2. Ridge regression analyses demonstrating the discriminatory value of DW-MRI parameters per week with pathologic complete response (TRG 1) as outcome variable after multiple imputation (20 datasets) of the missing ADC values in week 4 and 5.

\begin{tabular}{|c|c|c|c|c|c|c|c|}
\hline \multicolumn{4}{|l|}{ Full cohort $(n=24)$} & \multicolumn{4}{|l|}{ Sensitivity analyses $(n=20)^{\star}$} \\
\hline Intercept and predictors & $\beta$ & OR & $\begin{array}{l}\text { c- } \\
\text { statistic }\end{array}$ & Intercept and predictors & $\beta$ & OR & $\begin{array}{l}\text { c- } \\
\text { statistic }\end{array}$ \\
\hline \multicolumn{8}{|c|}{ Week 4} \\
\hline Intercept & -1.88 & & 0.80 & Intercept & -4.64 & & 0.93 \\
\hline$\Delta \mathrm{ADC}_{\text {week } 4}(\%)$ & 0.02 & 1.02 & & $\Delta \mathrm{ADC}_{\text {week } 4}(\%)$ & 0.08 & 1.08 & \\
\hline Squamous cell carcinoma ${ }^{q}$ & 0.65 & 1.92 & & Squamous cell carcinoma ${ }^{*}$ & 0.21 & 1.23 & \\
\hline \multicolumn{8}{|c|}{ Week 5} \\
\hline Intercept & -1.69 & & 0.72 & Intercept & -3.24 & & 0.87 \\
\hline$\Delta \mathrm{ADC}_{\text {week } 5}(\%)$ & 0.01 & 1.01 & & $\Delta \mathrm{ADC}_{\text {week } 5}(\%)$ & 0.04 & 1.04 & \\
\hline Squamous cell carcinoma ${ }^{*}$ & 0.66 & 1.94 & & Squamous cell carcinoma ${ }^{*}$ & 0.36 & 1.43 & \\
\hline
\end{tabular}

$A D C$ apparent diffusion coefficient; $c$-statistic concordance statistic; OR odds ratio; $p C R$ pathologic complete response

Note. Due to rounding, the reported odds ratios might not precisely correspond with the reported beta regression coefficients.

* After post-hoc exclusion of additional 4 patients based on baseline tumor volume delineated on DW-MRI $<7 \mathrm{ml}$ $(\mathrm{n}=3)$ and tumor histology other than adenocarcinoma or squamous cell carcinoma as based on the resection specimen $(n=1)$.

$¥$ Adenocarcinoma was used as reference category. 


\begin{tabular}{|c|c|c|c|c|c|c|c|c|c|c|c|c|c|c|c|c|c|c|}
\hline \multirow[b]{2}{*}{ Time in weeks } & \multicolumn{13}{|c|}{$\begin{array}{c}\text { Neoadjuvant } \\
\text { chemoradiotherapy }\end{array}$} & \multicolumn{5}{|c|}{$\begin{array}{l}\text { Surgical } \\
\text { resection }\end{array}$} \\
\hline & 0 & 1 & 2 & 3 & 4 & 5 & 6 & 7 & 8 & 9 & 10 & 11 & 12 & 13 & 14 & 15 & 16 & 17 \\
\hline
\end{tabular}

Supplementary Figure 1. Study design.

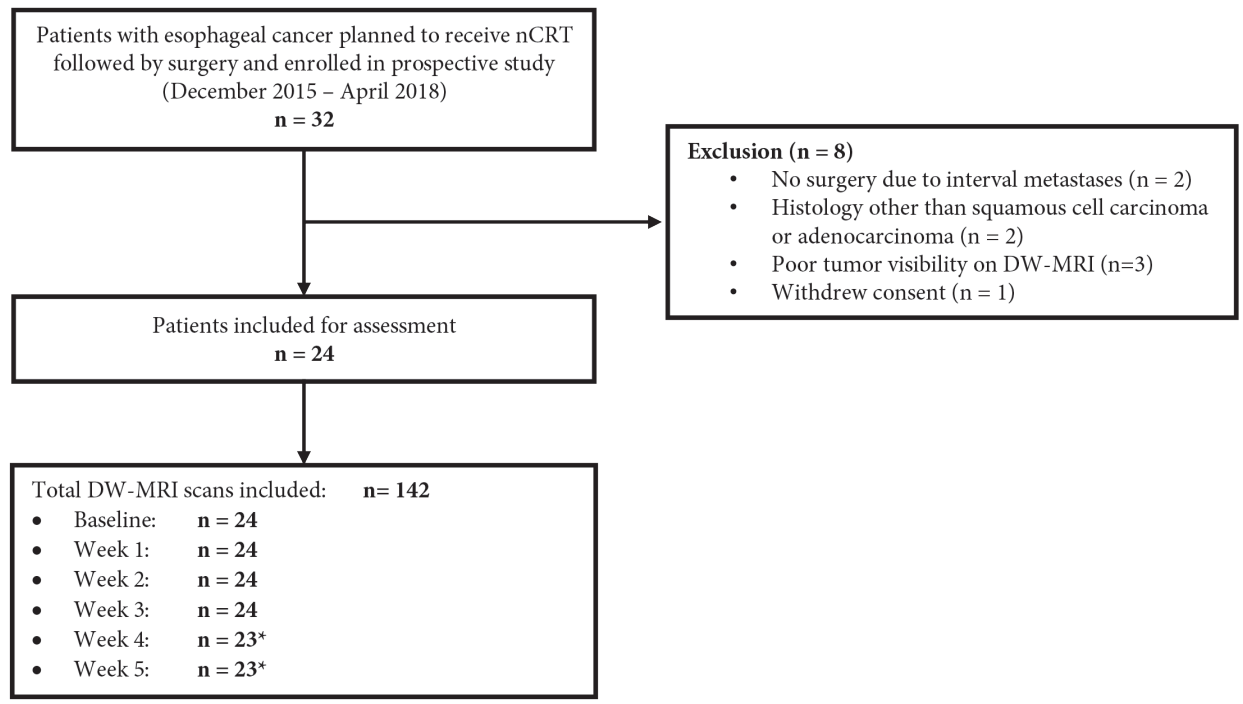

* Not available due to image acquisition problems $(\mathrm{n}=1)$ or patients' refusal $(\mathrm{n}=1)$

Supplementary Figure 2. Flowchart. 

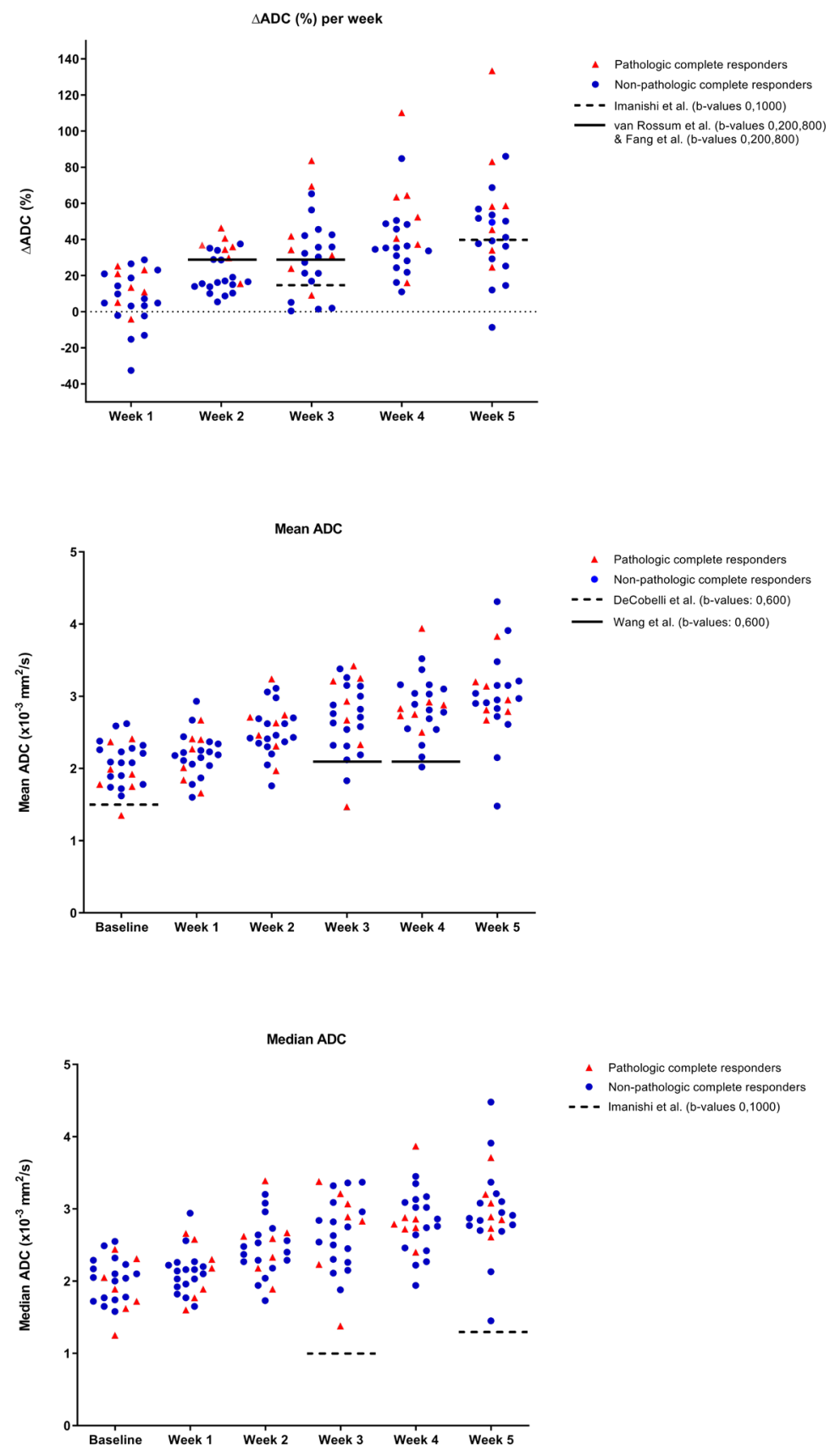

Supplementary Figure 3. Graphic illustration of $\triangle \mathrm{ADC}(\%)$ and $\mathrm{ADC}$ cut-off points derived from the available literature (represented by the dashed and solid horizontal lines) on DW-MRI in pathologic complete response assessment for esophageal cancer and applied to the current data. 


\section{References}

1. Lever FM, Lips IM, Crijns SPM, et al. Quantification of Esophageal Tumor Motion on Cine-Magnetic Resonance Imaging. Int J Radiat Oncol Biol Phys. 2014;88:419-424.

2. Pavlou M, Ambler G, Seaman SR, et al. How to develop a more accurate risk prediction model when there are few events. BMJ. 2015;351:h3868.

3. Sterne JAC, White IR, Carlin JB, et al. Multiple imputation for missing data in epidemiological and clinical research: potential and pitfalls. BMJ. 2009;338:b2393.

4. White IR, Royston P, Wood AM. Multiple imputation using chained equations: Issues and guidance for practice. Stat Med. 2011;30:377-399.

5. Rubin D. Multiple Imputation for Nonresponse in Surveys. John Wiley \& Sons Inc, Hoboken, New Jersey. 1987. 


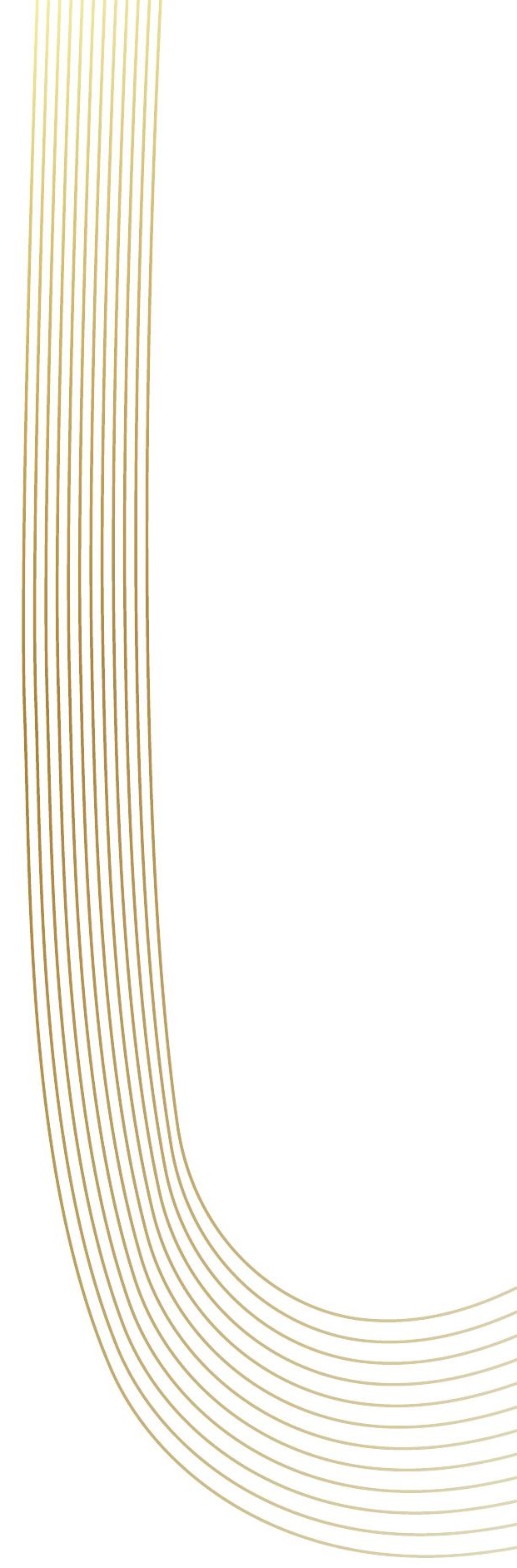




\section{Preoperative image-guided identification of response to neoadjuvant chemoradiotherapy in esophageal cancer (PRIDE): a multicenter observational study}

BMC Cancer 2018;18(1):1006.

A.S. Borggreve, S. Mook, M. Verheij, V.E.M. Mul, J.J. Bergman, A. Bartels-Rutten, L.C. ter Beek, R.G.H. Beets-Tan, R.J. Bennink, M.I. van Berge Henegouwen, L.A.A. Brosens, I.L. Defize, J.M. van Dieren, H. Dijkstra, R. van Hillegersberg, M.C. Hulshof, H.W.M. van Laarhoven, M.G.E.H. Lam, A.L.H.M.W. van Lier, C.T. Muijs, W.B. Nagengast, A.J. Nederveen, W. Noordzij, J.T.M. Plukker, P.S.N. van Rossum, J.P. Ruurda, J.W. van Sandick, B.L.A.M. Weusten, F.E.M. Voncken, D. Yakar, G.J. Meijer, on behalf of the PRIDE study group. 


\section{Abstract}

\section{Background}

Nearly one third of patients undergoing neoadjuvant chemoradiotherapy (nCRT) for locally advanced esophageal cancer have a pathologic complete response (pCR) of the primary tumor upon histopathological evaluation of the resection specimen. The primary aim of this study is to develop a model that predicts the probability of pCR to nCRT in esophageal cancer, based on diffusion-weighted magnetic resonance imaging (DW-MRI), dynamic contrastenhanced magnetic resonance imaging (DCE-MRI) and ${ }^{18} \mathrm{~F}$-fluorodeoxyglucose positron emission tomography with computed tomography $\left({ }^{18} \mathrm{~F}-\mathrm{FDG}\right.$ PET-CT). Accurate response prediction could lead to a patient-tailored approach with omission of surgery in the future in case of predicted pCR or additional neoadjuvant treatment in case of non-pCR.

\section{Methods}

The PRIDE study is a prospective, single arm, observational multicenter study designed to develop a multimodal prediction model for histopathological response to nCRT for esophageal cancer. A total of 200 patients with locally advanced esophageal cancer - of which at least 130 patients with adenocarcinoma and at least 61 patients with squamous cell carcinoma - scheduled to receive nCRT followed by esophagectomy will be included. The primary modalities to be incorporated in the prediction model are quantitative parameters derived from MRI and ${ }^{18} \mathrm{~F}-\mathrm{FDG}$ PET-CT scans, which will be acquired at fixed intervals before, during and after nCRT. Secondary modalities include blood samples for analysis of the presence of circulating tumor DNA (ctDNA) at 3 time-points (before, during and after nCRT), and an endoscopy with (random) bite-on-bite biopsies of the primary tumor site and other suspected lesions in the esophagus as well as an endoscopic ultrasonography (EUS) with fine needle aspiration of suspected lymph nodes after finishing nCRT. The main study endpoint is the performance of the model for pCR prediction. Secondary endpoints include progression-free and overall survival.

\section{Discussion}

If the multimodal PRIDE concept provides high predictive performance for $\mathrm{pCR}$, the results of this study will play an important role in accurate identification of esophageal cancer patients with a pCR to nCRT. These patients might benefit from a patient-tailored approach with omission of surgery in the future. Vice versa, patients with non-pCR might benefit from additional neoadjuvant treatment, or ineffective therapy could be stopped.

Trial registration: The article reports on a health care intervention on human participants and was prospectively registered on March 22, 2018 under ClinicalTrials.gov Identifier: NCT03474341. 


\section{Background}

Esophageal cancer is the ninth most common type of cancer and the sixth most leading cause of cancer related death. ${ }^{1}$ Surgical resection has long been the standard curative treatment for locally advanced esophageal cancer. However, the poor survival rates of surgery alone prompted many researchers to explore neoadjuvant therapy approaches to improve survival. Randomized clinical trials have demonstrated a consistent prognostic benefit of neoadjuvant chemotherapy or chemoradiotherapy followed by surgery over surgery alone for locally advanced esophageal cancer. ${ }^{2-4}$ In the Netherlands, this resulted in the adoption of neoadjuvant chemoradiotherapy (nCRT) according to the CROSS regimen followed by surgery as standard of care. ${ }^{4}$

Nearly one third of all esophageal cancer patients (29\%) treated with nCRT have no viable tumor cells detected at the primary tumor site at histopathological evaluation of the resection specimen, referred to as pathologic complete response (pCR). ${ }^{4}$ It has been argued that in patients who achieve a $\mathrm{pCR}$, surgery may be omitted without substantially reducing survival outcomes. In fact, as an esophagectomy is associated with substantial morbidity, mortality (up to 3-5\%) and impaired quality of life ${ }^{5-9}$, it can be speculated that surgery may have a detrimental effect on these patients. Consequently, proper identification of pathologic complete responders prior to surgery could yield an organ-preserving regimen avoiding esophagectomy and its postoperative complications.

Reversely, $18 \%$ of patients have more than $50 \%$ vital residual tumor cells in the primary tumor bed at histopathology after nCRT and surgery, referred to as non-responders. ${ }^{4}$ The CROSS regimen is associated with grade $\geq 3$ toxicity events according to the Common Terminology Criteria for Adverse Events (CTCAE) in up to $13 \%$ of patients. ${ }^{4}$ Thus, these non-responders are exposed to side effects of nCRT probably without the benefits. Therefore, early identification of the non-responders during nCRT may be beneficial, as alternative treatment strategies could be explored for this group, such as additional neoadjuvant treatment, or ineffective therapy could be stopped.

Several diagnostic strategies have been proposed to predict response and ultimately omit surgery in selected patients. Computed tomography (CT) is used preferably in initial staging of esophageal cancer, especially with regard to the presence of distant metastases, but does not satisfactorily restage after nCRT (accuracies ranging from 51-75\%). ${ }^{10-12}$ Remaining tumor tissue is difficult to distinguish from therapy-induced peritumoral fibrosis and inflammation. As such, CT tends to overstage the preoperative tumor status.

Endoscopic ultrasonography (EUS) with or without biopsy has not yielded satisfactory results either. Systematic reviews pointed out that the accuracy rates of EUS for evaluating response to nCRT in esophageal cancer were moderate to poor (27-78\%). ${ }^{10,12,13}$ The pooled sensitivity of EUS after nCRT for detection of residual primary tumor in a meta-analysis 
including 11 studies was $96.4 \%$ (95\%-CI: 91.7\%-98.5\%), with a pooled specificity of only 10.9\% (95\%-CI: 3.5\%-29.0\%). ${ }^{14}$ Endoscopic biopsy after chemoradiotherapy for esophageal cancer on the other hand was a very specific (pooled estimate $91.0 \%, 95 \%-\mathrm{CI}$ : 85.6\%-94.5\%), but not a sensitive method (pooled estimate 34.5\%, 95\%-CI: 26.0\%-44.1\%) for detection of residual primary tumor after nCRT, as reported in a recent meta-analysis including 12 studies. ${ }^{14}$ Results from the recently published preSANO study revealed that with bite-on-bite biopsies, the sensitivity for the detection of residual disease increased substantially compared to regular biopsies (an increase from 54\% [95\%-CI: 38\%-70\%] to $74 \%$ [95\%-CI: $64 \%-83 \%]) .{ }^{15}$

Moreover, promising results for response prediction were obtained using repeated integrated ${ }^{18} \mathrm{~F}$-fluorodeoxyglucose positron emission tomography and computed tomography $\left({ }^{18} \mathrm{~F}-\mathrm{FDG}\right.$ PET-CT), with accuracies ranging from $76 \%$ to $85 \% .{ }^{10,16-18}$ The change in ${ }^{18} \mathrm{~F}$-FDG uptake during nCRT, reflecting a change in glucose metabolism by cancer cells, may be used to identify these responders. ${ }^{16} \mathrm{~A}$ systematic review on the value of these quantitative ${ }^{18} \mathrm{~F}$-FDG PET(-CT) measurements including 20 studies, showed that response could be predicted with sensitivities ranging from $33 \%$ to $100 \%$ (pooled estimate of $67 \%$ ) and specificities ranging from $30 \%$ to $100 \%$ (pooled estimate of $68 \%$ ). ${ }^{16}$

This supports the concept that functional imaging could play an important role in accurate response prediction. In this light, magnetic resonance imaging (MRI) has recently shown great potential for response prediction to nCRT for esophageal cancer. ${ }^{19-22}$ Diffusionweighted MRI (DW-MRI) is a functional imaging modality that allows for tissue characterization by deriving image contrast from restriction in the free diffusion (i.e. random mobility or Brownian motion) of water molecules, which is related to microstructural tissue organization. An apparent diffusion coefficient (ADC) map can be derived from the DW-MRI images to quantify the diffusion restriction in a certain volume of interest. The ADC is inversely correlated with tissue cellularity. As chemoradiotherapy can result in the loss of cell membrane integrity, tumor response can be detected as an increase in tumor $\mathrm{ADC}$. In two exploratory studies, the treatment-induced relative changes in $\mathrm{ADC}$ over time $(\triangle \mathrm{ADC})$, during $\mathrm{nCRT}$, appeared highly predictive of histopathological response. ${ }^{21,23}$ Using repeated DW-MRI only, a high area under the receiver operating curve $\left(\mathrm{AUC}_{\mathrm{ROC}}\right)$ was attained for identifying pathologic complete responders. ${ }^{21,23}$

Dynamic contrast-enhanced magnetic resonance imaging (DCE-MRI), the acquisition of serial MR images while intravenously administering a contrast agent, provides further insight into the nature of the tissue properties related to perfusion. Based on these images, quantitative parameters such as the transfer constant $\left(\mathrm{K}^{\text {trans }}\right)$ and blood-normalized initialarea-under-the-gadolinium-concentration curve (AUC) can be calculated. The AUC reflects blood flow, vascular permeability and the fraction of interstitial space. ${ }^{24}$ In a pilot study, all pathologic complete responders showed a decrease in AUC of $25 \%$ or more over the entire 
treatment course ( $\triangle \mathrm{AUC})$, whereas an increase in AUC during treatment was observed for those patients who did not obtain a pCR $(p=0.003){ }^{25}$

In addition to functional imaging, circulating tumor cells and corresponding circulating tumor DNA (ctDNA) have been proposed as noninvasive and real-time biomarkers for predicting patient prognosis in esophageal carcinomas. ${ }^{26-29}$ Circulating tumor cells and ctDNA are present in the blood vessels adjacent to the tumor, and are subsequently transported throughout the body via the circulation. ${ }^{28}$ As such, ctDNA reflects the presence of disease and could provide valuable information on the response to treatment. Since ctDNA can be detected from regular peripheral blood samples, the detection of ctDNA could be a promising, minimally invasive addition to the evaluation of treatment response and prognosis in esophageal cancer patients.

\section{Study aim}

As the aforementioned modalities do not individually fulfill the requirements to justify treatment decision making, the primary aim of the current study is to develop a multimodal prediction model that predicts the patients' individual probability of a pCR after nCRT for esophageal cancer. Accurate prediction of the response to nCRT could lead to a patienttailored approach with omission of surgery in the future in case of predicted PCR, potentially improving quality of life and reducing health care costs. Furthermore, additional neoadjuvant treatment could be offered to patients in case of non-pCR.

\section{Methods}

\section{Objectives}

The primary objective of the study is to develop a multimodal prediction model that predicts a patients' individual probability of a pathologic complete response to nCRT in esophageal cancer by integrating DW-MRI, DCE-MRI and ${ }^{18} \mathrm{~F}-\mathrm{FDG}$ PET-CT scans acquired prior to, during and after administration of nCRT.

The secondary objectives are as follows:

- To evaluate the accuracy of the multimodal prediction model as developed under the primary objective for the prediction of a pathologic good response (i.e. tumor regression grade [TRG] 1 or TRG2).

- To evaluate the effectiveness and efficacy of an endoscopic and endosonographic assessment after nCRT for the detection of residual disease, in relation to the response classification as predicted by the model developed under the primary objective.

- To evaluate the presence of, and changes in, ctDNA during nCRT as a biomarker for a patients' response to nCRT, the detection of residual disease after nCRT and progressionfree and overall survival. 
- To evaluate the accuracy of the multimodal prediction model as developed under the primary objective with addition of the endoscopic and endosonographic assessment, and the ctDNA measurements for the prediction of $\mathrm{pCR}$ and pathologic good response (i.e. TRG1 or TRG2).

- To evaluate the accuracy of a visual assessment for the detection of residual disease after nCRT based on MRI and ${ }^{18} \mathrm{~F}-\mathrm{FDG}$ PET-CT.

- To evaluate the performance of MRI and ${ }^{18} \mathrm{~F}$-FDG PET-CT imaging parameters for the prediction of progression-free and overall survival.

\section{Study design}

The PRIDE study is a prospective, multi-center observational study with participation of 4 high-volume centers in the Netherlands (University Medical Center Utrecht, The Netherlands Cancer Institute - Antoni van Leeuwenhoek Hospital, University Medical Center Groningen and Amsterdam University Medical Centers). Patients will be informed and included at the outpatient department at one of these investigational centers. The study has been approved by the Medical Ethics Review Committee of the University Medical Center Utrecht (17-941, NL62881.041.17). All participating hospitals gave their consent after assessment of local feasibility. Written, voluntary, informed consent to participate in the study will be obtained from all patients.

\section{Study population}

In order to be eligible to participate in this study, a patient must be scheduled to receive nCRT for a potentially resectable, locally advanced (cT1b-4aN0-3M0) esophageal or gastroesophageal junction tumor, either squamous cell carcinoma or adenocarcinoma. Neoadjuvant chemoradiotherapy will be delivered according to the CROSS-regimen ${ }^{4}$, consisting of weekly administration of carboplatin (doses titrated to achieve an area under the curve of $2 \mathrm{mg}$ per milliliter per minute) and paclitaxel (50 mg per square meter of body-surface area) for 5 weeks and concurrent radiotherapy (41.4 Gy in 23 fractions, delivered 5 days per week on workdays with intensity modulated radiotherapy) followed by esophagectomy after 8-10 weeks. Primary diagnosis and staging will be based on endoscopy, EUS, ${ }^{18} \mathrm{~F}-\mathrm{FDG}$ PET-CT and histopathological evaluation of a tumor biopsy.

Patients who meet exclusion criteria for MRI or for intravenous gadolinium-based contrast, patients with a blood plasma glucose concentration $>10 \mathrm{mmol} / \mathrm{L}$ or poorly controlled diabetes mellitus, patients with a status after endoscopic mucosal resection (EMR) or endoscopic submucosal dissection (ESD) of the primary tumor prior to the start of nCRT, patients younger than 18 years and pregnant or breast-feeding patients are not eligible. 


\section{Study protocol}

A schematic representation of the study protocol is depicted in Figure 1. Patients will undergo standard diagnostic work-up and staging for esophageal cancer, including a baseline ${ }^{18} \mathrm{~F}$-FDG PET-CT (PET-CT ${ }_{\text {pre }}$ ). After informed consent and before the start of nCRT, a baseline MRI (MRI $\left.{ }_{\text {pre }}\right)$ is performed. A second MRI (MRI $\left.{ }_{\text {per }}\right)$ and ${ }^{18} \mathrm{~F}-\mathrm{FDG}$ PET-CT $\left(\right.$ PET-CT $_{\text {per }}$ ) will be performed during the third week of nCRT (after 10-15 fractions of radiotherapy). A third MRI (MRI ${ }_{\text {post }}$ ) will be performed 6-8 weeks after the completion of nCRT, and no sooner than within 2 weeks before the intended date of surgery. The third ${ }^{18} \mathrm{~F}-\mathrm{FDG}$ PET-CT (PET-CT $\mathrm{Post}_{\text {) }}$ is usual care in all participating centers, and will be performed within the same timeframe as the MRI $I_{\text {post }}$. Blood samples will be acquired at 3 time points, i.e. before, during and after nCRT, to evaluate the presence of, and changes in circulating tumor DNA (ctDNA). Furthermore, patients will be asked to undergo an additional endoscopic assessment after nCRT, PET-CT $\mathrm{p}_{\text {post }}$ and $\mathrm{MRI}_{\text {post }}$, within 2 weeks prior to surgery. Surgical resection will be performed 8-10 weeks after completion of nCRT.

In summary, for study purposes patients will undergo 3 additional MRI scans (MRI ${ }_{\text {pre' }}$ MRI $_{\text {per }}$ MRI $\left.{ }_{\text {post }}\right), 1$ additional ${ }^{18} \mathrm{~F}$-FDG PET-CT scan (PET-CT ${ }_{\text {per }}$ ), blood samples at 3 time points and 1 postchemoradiation endoscopic and endosonographic assessment. The ${ }^{18} \mathrm{~F}-\mathrm{FDG}$ PET-CT scans before start of nCRT and after nCRT (PET-CT $\mathrm{pre}_{\text {and PET-CT }}$ post are standard of care in all participating centers and will also be used for study purposes. All study related procedures will take place before surgery.

\section{MRI}

Patients will undergo anatomical (T2-weighted [T2W]) and functional MRI (DWI and DCE) in one scanning session every time. Two DWI series and one DCE scan will be acquired. The sagittal DWI series (sagittal intravoxel incoherent motion [sIVIM] with 13 b-values: $0,10,20,30,40,50,75,100,200,350,500,650$ and $800 \mathrm{~s} / \mathrm{mm}^{2}$ ) will be used for quantitative analyses but also for the visual assessment. Transversal DWI series (high resolution tDWI, b-values: 0 and $800 \mathrm{~s} / \mathrm{mm}^{2}$ ) will mostly be used for visual assessments. The DCE-MRI scans will be acquired with a temporal resolution of 3 seconds and the injection of a gadolinium-based contrast agent. ADC and AUC values of the various time points will be used as quantitative measures of the DWI and DCE series, respectively. Extensive effort has been put in the standardization of MRI scan sequences by imaging experts and the exchange of test scans.

\section{${ }^{18}$ F-FDG PET-CT}

The PET-CT examinations will be performed according to EARL guidelines (European Association of Nuclear Medicine $)^{30} .{ }^{18} \mathrm{~F}-\mathrm{FDG}$ is the tracer that will be used for the assessment of abnormal glucose metabolism in the tumor. On the ${ }^{18} \mathrm{~F}-\mathrm{FDG}$ PET-CT scans, standardized 


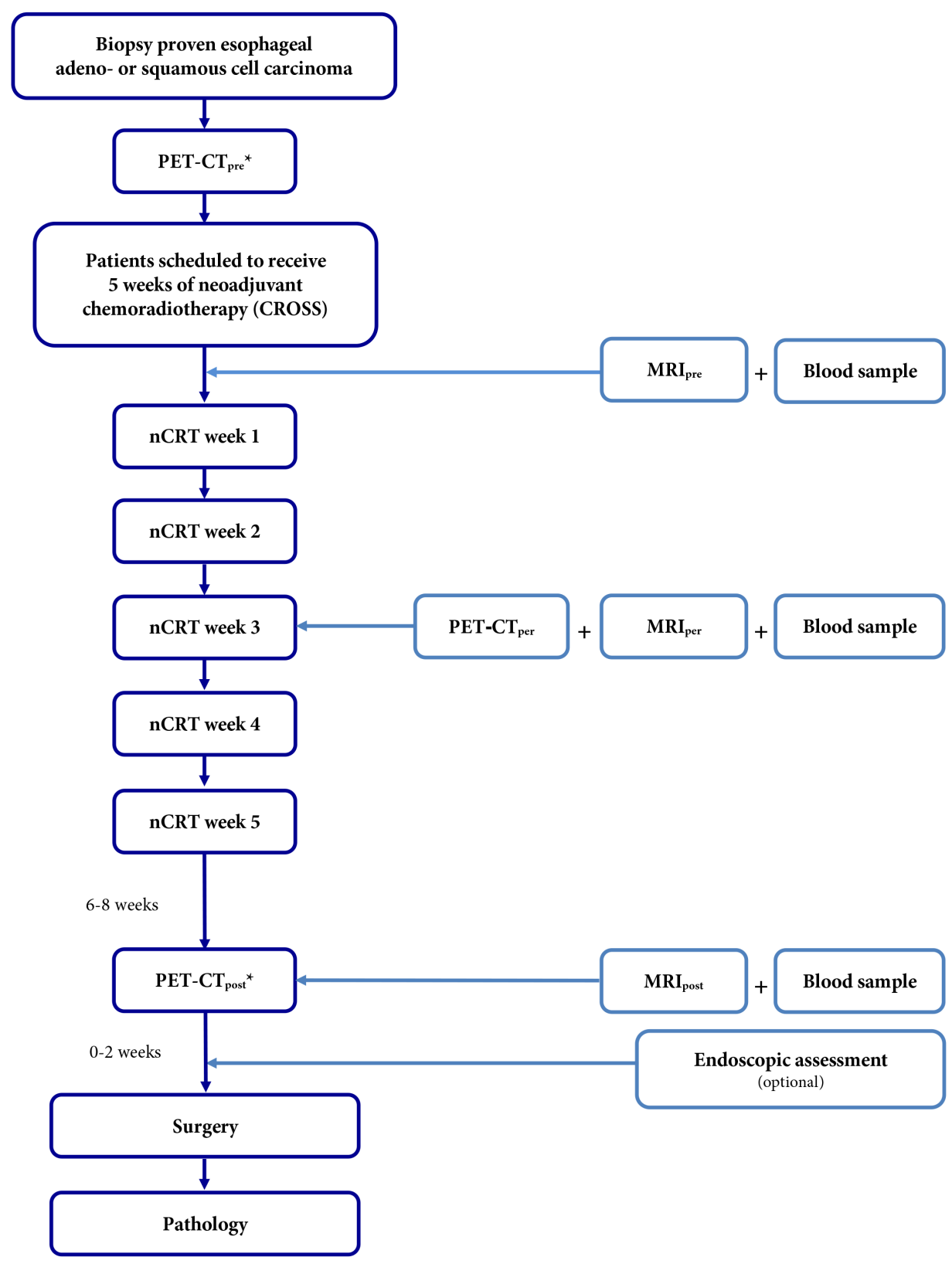

* PET-CT pre $_{\text {and PET-CT }}$ post are usual care but will be used for study purposes

Figure 1. Study design 
uptake volumes $\left(\mathrm{SUV}_{\max }, \mathrm{SUV}_{\text {mean }}\right.$ ) and the total lesion glycolysis (TLG) will be measured to quantify changes over time in the glucose metabolism of the tumor.

\section{Postchemoradiation endoscopic assessment}

Patients will be asked to undergo a postchemoradiation endoscopic assessment, consisting of an additional endoscopy with (random) bite-on-bite biopsies of the primary tumor site and other suspected lesions in the esophagus, as well as endosonography with fine needle aspiration of suspected lymph nodes after completion of nCRT. This is an optional study procedure and patients can choose to opt-out for this additional procedure.

The endoscopic reevaluation will be performed by 1 or 2 experts in each of the 4 centers, to ensure high quality and uniform procedures and to reduce the impact of operator dependency. Furthermore, video recordings of all patients with negative biopsies that showed visual abnormalities of any kind during the endoscopic procedure will be reevaluated by an expert panel that will be blinded for the pathological outcome of the resection specimen in order to investigate whether a qualitative assessment of an expert team can help to correctly identify residual tumor in patients with a negative biopsy.

\section{Blood samples}

Blood samples will be used to evaluate the presence of ctDNA and changes in ctDNA concentrations since the release of ctDNA within a patient during the course of chemoradiotherapy has recently been demonstrated to be a dynamic process. ${ }^{31}$ To allow molecular analysis of liquid biopsies, blood will be collected in cell-free DNA collection tubes. The plasma will be aliquoted after 2 centrifugation steps and will be stored at $-80^{\circ} \mathrm{C} .{ }^{32}$ This will allow isolation of ctDNA and subsequent mutation analysis by means of Next Generation Sequencing (NGS) at a later stage.

\section{Surgery}

A transthoracic or transhiatal esophagectomy will be performed in all patients, depending on patient characteristics, tumor localization, and local preference. Open, hybrid and completely minimally invasive techniques are allowed. Resection of the primary tumor and regional lymph nodes will be carried out according to the current requirements for esophageal cancer surgery in The Netherlands..$^{33}$ For correct TNM-staging, the lymph node dissection should contain at least 15 nodes derived from both the mediastinum and upper abdomen.

\section{Histopathological assessment}

The resection specimen will be evaluated meticulously according to a standardized protocol (tumor type and extension, lymph nodes, resection margins) by a dedicated pathologist with gastrointestinal subspecialty in each center. The pathologist will be blinded for the 
results of the MRI and PET-CT exams. The most recent edition of the UICC (International Union Against Cancer) protocol will be used for TNM-classification and stage grouping. ${ }^{34}$ Special attention will be given to reporting the effects of nCRT in the resection specimen. The (estimated) location of the primary lesion plus surrounding areas and other suspected lesions in the esophagus will be embedded in order to adequately judge the presence of residual tumor and treatment effects. The percentage of viable tumor cells will be scored microscopically (ranging from 0-100\%), which directly corresponds to a stage in either of the two most often used grading systems: 'TRG 1 to $4^{35}$ or the 'Mandard score 1 to $5^{336}$. Therapy effects include necrosis, inflammation with multinucleated giant cells, fibrosis and calcifications. Fibrosis is the most remarkable effect and is used to estimate the extension of the tumor before treatment. Lastly, all resection specimens with TRG 1-2 will be revised by a second expert pathologist.

\section{Follow-up}

Patients will remain in follow-up for five years after surgery, according to local follow-up policies. The general follow-up guideline in the Netherlands consists of routine follow-up visits every 3 months during the first year after surgery. In the second year, follow-up takes place every 6 months, and then yearly until 5 years after surgery. Diagnostic investigations are generally only performed on indication. ${ }^{33}$

\section{Study outcomes}

The primary outcome of this study is the performance of the multimodal prediction model for the correct prediction of a patients' individual probability of a pCR to nCRT based on DW-MRI, DCE-MRI and ${ }^{18}$ F-FDG PET-CT scans acquired prior to, during and after administration of nCRT. Secondary outcome parameters include the performance of the model for good response (i.e. TRG1 and TRG2), the effectiveness and efficacy of a postchemoradiation endoscopic and endosonographic assessment for pCR prediction, the value of ctDNA as a biomarker for a patients' response to nCRT, progression-free and overall survival, the performance of the model including results from the endoscopic and endosonographic assessment and ctDNA measurements for pCR and good response prediction, the performance of a visual assessment for the detection of $\mathrm{pCR}$ after nCRT based on MRI and ${ }^{18} \mathrm{~F}-\mathrm{FDG}$ PET-CT, and lastly the performance of the model for prediction of progression-free and overall survival.

\section{Statistical analysis}

Data analysis of primary study objective

The analysis regarding the primary objective of this project will have pCR as the predicted outcome of interest. Statistical analysis and reporting will be performed in accordance with 
the Standards for the Reporting of Diagnostic accuracy studies (STARD) statement, and the Transparent Reporting of a multivariable prediction model for Individual Prognosis or Diagnosis (TRIPOD) statement ${ }^{37,38}$. The assessor(s) of the MRI and PET-CT images will be blinded for the histopathological outcome.

A multivariable logistic regression model will be developed with $\mathrm{pCR}$ as dichotomous outcome measure. Many of the imaging parameters are likely highly correlated and provide similar (non-additional) information, particularly within one modality. The most valuable imaging parameters within one imaging modality (i.e. within DW-MRI, DCE-MRI and PET-CT imaging parameters) will be entered in the model, based on the results of previous knowledge. To determine whether the imaging modalities provide complementary value in the prediction of pCR, models will be compared based on Akaike's Information Criterion (AIC).

Model discrimination and calibration results will be evaluated for the multivariable logistic regression models using receiver operating characteristic (ROC) curve analysis with areaunder-the-curve $\left(\mathrm{AUC}_{\mathrm{ROC}}\right)$ estimates and visual inspection of model calibration plots, respectively. Internal validation using the bootstrap method with 1000 repetitions will be carried out to provide insight into potential over-fitting and optimism in model performance. Bootstrapping will allow for calculation of bias-corrected c-indexes of the prediction model, and provides shrinkage factors that can be used to adjust the estimated regression coefficients in the model for overfitting and miscalibration. Sensitivity analyses will be performed excluding one participating center each time to study the influence of the multicenter study design on the model performance.

\section{Data analysis of secondary study objectives}

ROC curve analysis with $\mathrm{AUC}_{\mathrm{ROC}}$ estimates will be used to determine the additional value of the postchemoradiation endoscopic and endosonographic assessment and the ctDNA measurements to the model as developed under the primary objective, as well as the accuracy of the multimodal prediction model for the prediction of pathologic good response (i.e. combined TRG1 and TRG2).

The performance of a visual assessment for the detection of pCR after nCRT will be analyzed by calculation of diagnostic performance measures such as sensitivity, specificity, positive predictive value, negative predictive value and accuracy (including corresponding 95\% confidence intervals). This also applies to the individual performance of the endoscopic and endosonographic assessment for the detection residual disease, as well as for the performance of ctDNA measurements.

Multivariable Cox regression models will be used to analyze the performance of the prediction model as developed under the primary objective, MRI and ${ }^{18} \mathrm{~F}$-FDG PET-CT imaging parameters, and ctDNA measurements for the prediction of progression-free and overall survival. 


\section{Sample size calculation}

It is conservatively assumed that adenocarcinoma and squamous cell carcinoma need separate modeling and a priori stratification. Based on 3 independent imaging predictors (e.g. a DW-MRI imaging parameter such as $\triangle \mathrm{ADC}$, a DCE-MRI imaging parameter such as $\triangle \mathrm{AUC}$ and a ${ }^{18} \mathrm{~F}$-FDG PET-CT imaging parameter such as $\triangle \mathrm{SUV}_{\max }$ ), this requires 30 events for both histopathological subtypes, according to the ' 1 predictor per $\sim 10$ events' rule-of-thumb in logistic regression analysis. ${ }^{39,40}$ The CROSS-trial demonstrated a pCR rate of $23 \%$ and $49 \%$ after nCRT for patients with adenocarcinomas and squamous-cell carcinomas respectively. ${ }^{4}$ According to the 1 in 10 rule, this translates in a total accrual of at least 130 adenocarcinoma patients and at least 61 squamous-cell carcinoma patients. In case of an unexpected aberrant distribution of patients that leads to decreased pCR rates, the aim is an accrual of 200 patients.

\section{Discussion}

Currently, groups of patients with esophageal cancer fit in certain protocolled treatment approaches, but the treatment is rarely a perfect fit for the individual patient. The PRIDE study investigates whether a multimodal image-guided model can be developed that accurately predicts a patients' individual histopathological response to nCRT. Such a model would enable personalized treatment for patients with esophageal cancer. Recent studies indicate that an organ-sparing approach might be feasible in selected patients with esophageal cancer who have a pCR after nCRT. ${ }^{41-43}$ However, satisfactory diagnostic strategies to select these pathologic complete responders are lacking up to now. Therefore, surgical resection after nCRT remains the most optimal curative treatment in terms of survival in patients with locally advanced esophageal cancer. If the PRIDE concept provides high predictive performance for $\mathrm{PCR}$, this could potentially lead to a new standard of care with direct benefits to esophageal cancer patients. Furthermore, accurate identification of the non-responders may be beneficial, as these patients might benefit from alternative treatment strategies, such as additional neoadjuvant treatment, or ineffective therapy could be stopped in this group.

In the current study protocol, strict time points are chosen for the MRI and ${ }^{18} \mathrm{~F}-\mathrm{FDG}$ PETCT imaging, as well as for the blood samples and endoscopic assessment. This way, a homogeneous cohort will be created, in which measurement variability will be reduced as much as possible. This is also reflected in the extensive effort of the participating centers to standardize the imaging protocols.

Since therapy effects continue to develop after treatment, previous studies have underlined that MRI and PET-CT imaging during nCRT as well as after nCRT for esophageal cancer 
can function as predictors for pCR. ${ }^{10,18,25,21,44,45}$ As such, the MRI ${ }_{\text {post }}$ and PET-CT post scans should be as close to the histopathological assessment of the outcome (pCR) as possible, in order to make sure that the findings on the MRI ${ }_{\text {post }}$ and PET-CT ${ }_{\text {post }}$ will represent the histopathology accurately. This will likely also prevent false positive results caused by transient radiation-induced esophagitis, which is known to decrease over time after nCRT. Therefore, the chosen time points in our study include scans before the start of nCRT

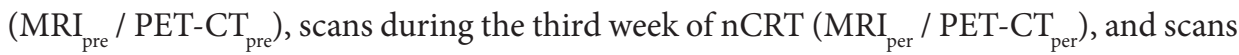
within 2 weeks before surgery $\left(\mathrm{MRI}_{\text {post }} / \mathrm{PET}^{-\mathrm{CT}_{\text {post }}}\right)$. In patients undergoing an additional endoscopic and endosonographic assessment, this will be intended after the ${ }^{18} \mathrm{~F}-\mathrm{FDG}$ PET$\mathrm{CT}_{\text {post }}$ and the MRI $\mathrm{post}$, but prior to surgery. If an organ-sparing approach is eventually implemented in clinical practice for predicted complete responders, an endoscopic confirmation without signs of residual tumor will most likely be required.

Of note, two studies, namely the Dutch SANO trial ${ }^{46}$ and the French ESOSTRATE trial (ClinicalTrials.gov identifier NCT02551458), are currently studying active surveillance strategies after nCRT for patients with a clinical complete response. For the SANO trial, a clinical complete response is based on ${ }^{18} \mathrm{~F}$-FDG PET-CT and endoscopy with at least 8 (random) bite-on-bite biopsies. These studies together will include a total of 600 patients (300 within each trial) and the primary outcome of these studies is survival. In contrast to these trials, the current study involves the careful development of an accurate image-guided response evaluation strategy to predict $\mathrm{pCR}$ in an observational study, without the simultaneous implementation of postponed surgical resection in clinical complete responders that might harm the patient. The results of this study will therefore play an important role in the accurate identification of esophageal cancer patients with a pCR to nCRT who could benefit from an organ-sparing approach in the future. Ultimately, the results of the three trials together could lead to a patient-tailored wait-and-see approach with omission of surgery in the appropriate patients. 


\section{References}

1. Fitzmaurice C, Dicker D, Pain A, et al. The Global Burden of Cancer 2013. JAMA Oncol. 2015;1(4):505.

2. Cunningham D, Allum WWH, Stenning SSP, et al. Perioperative chemotherapy versus surgery alone for resectable gastroesophageal cancer. N Engl J Med. 2006;355(1):11-20.

3. Ychou M, Boige V, Pignon J, et al. Perioperative chemotherapy compared with surgery alone for resectable gastroesophageal adenocarcinoma: an FNCLCC and FFCD multicenter phase III trial. J Clin Oncol. 2011;29(13):1715.

4. Hagen P, Hulshof MCCMC, Lanschot JJ, et al. Preoperative Chemoradiotherapy for Esophageal or Junctional Cancer. N Engl J Med. 2012;366(22):2074-2084.

5. Djärv T, Lagergren J, Blazeby JM, Lagergren P. Long-term health-related quality of life following surgery for oesophageal cancer. Br J Surg. 2008;95(9):1121-1126.

6. Mc Cormack O, Zaborowski A, King S, et al. New-onset Atrial Fibrillation Post-surgery for Esophageal and Junctional Cancer Incidence, Management, and Impact on Short-and Long-term Outcomes. Ann Surg. 2014;260(5):772-778.

7. Kassis ES, Kosinski AS, Ross P, Koppes KE, Donahue JM, Daniel VC. Predictors of anastomotic leak after esophagectomy: An analysis of the society of thoracic surgeons general thoracic database. Ann Thorac Surg. 2013;96(6):1919-1926.

8. Busweiler LAD, Wijnhoven BPL, van Berge Henegouwen MI, et al. Early outcomes from the Dutch Upper Gastrointestinal Cancer Audit. Br J Surg. 2016;103(13):1855-1863.

9. Schandl A, Lagergren J, Johar A, Lagergren P. Health-related quality of life 10 years after oesophageal cancer surgery. Eur J Cancer. 2016;69:43-50.

10. Westerterp M, van Westreenen HL, Reitsma JB, et al. Esophageal Cancer: CT, Endoscopic US, and FDG PET for Assessment of Response to Neoadjuvant Therapy-Systematic Review. Radiology. 2005;236(3):841851.

11. van Heijl M, Phoa SSKS, van Berge Henegouwen MI, et al. Accuracy and reproducibility of 3D-CT measurements for early response assessment of chemoradiotherapy in patients with oesophageal cancer. Eur J Surg Oncol. 2011;37(12):1064-1071.

12. Yip C, Cook GJR, Landau DB, Davies A, Goh V. Performance of different imaging modalities in assessment of response to neoadjuvant therapy in primary esophageal cancer. Dis Esophagus. 2016;29(2):116-130.

13. Ngamruengphong S, Sharma VK, Nguyen B, Das A. Assessment of response to neoadjuvant therapy in esophageal cancer: an updated systematic review of diagnostic accuracy of endoscopic ultrasonography and fluorodeoxyglucose positron emission tomography. Dis Esophagus. 2010;23(3):216-231.

14. van Rossum PSN, Goense L, Meziani J, et al. Endoscopic biopsy and EUS for the detection of pathologic complete response after neoadjuvant chemoradiotherapy in esophageal cancer: a systematic review and meta-analysis. Gastrointest Endosc. 2016;83(5):866-879.

15. Noordman BJ, Spaander MCW, Valkema R, et al. Detection of residual disease after neoadjuvant chemoradiotherapy for oesophageal cancer (preSANO): a prospective multicentre, diagnostic cohort study. Lancet Oncol.2018;19(7):965-974.

16. Kwee RM. Prediction of Tumor Response to Neoadjuvant Therapy in Patients with Esophageal Cancer with Use of ${ }^{18}$ F FDG PET: A Systematic Review. Radiology. 2010;254(3):707-717.

17. Roedl JB, Halpern EF, Colen RR, Sahani D V, Fischman AJ, Blake MA. Metabolic tumor width parameters as determined on PET/CT predict disease-free survival and treatment response in squamous cell carcinoma of the esophagus. Mol Imaging Biol. 2009;11(1):54-60.

18. van Heijl M, Omloo JM, van Berge Henegouwen MI, et al. Fluorodeoxyglucose Positron Emission Tomography for Evaluating Early Response During Neoadjuvant Chemoradiotherapy in Patients With Potentially Curable Esophageal Cancer. Ann Surg. 2011;253(1):56-63.

19. Aoyagi T, Shuto K, Okazumi S, Shimada H, Kazama T, Matsubara H. Apparent diffusion coefficient values measured by diffusion-weighted imaging predict chemoradiotherapeutic effect for advanced esophageal cancer. Dig Surg. 2011;28(4):252-257.

20. De Cobelli F, Giganti F, Orsenigo E, et al. Apparent diffusion coefficient modifications in assessing gastrooesophageal cancer response to neoadjuvant treatment: Comparison with tumour regression grade at histology. Eur Radiol. 2013;23(8):2165-2174. 
21. van Rossum PS, van Lier AL, van Vulpen M, et al. Diffusion-weighted magnetic resonance imaging for the prediction of pathologic response to neoadjuvant chemoradiotherapy in esophageal cancer. Radiother Oncol. 2015;115(2):163-170.

22. Wang L, Liu L, Han C, et al. Chemoradiotherapy of esophageal cancer The diffusion-weighted magnetic resonance imaging (DWI) predicts the early response of esophageal squamous cell carcinoma to concurrent chemoradiotherapy. Radiother Oncol. 2016;121:246-251.

23. Fang P, Musall BC, Son JB, et al. Multimodal Imaging of Pathologic Response to Chemoradiation in Esophageal Cancer. Int J Radiat Oncol. 2018;102(4):996-1001.

24. Evelhoch JL, LoRusso PM, He Z, et al. Magnetic resonance imaging measurements of the response of murine and human tumors to the vascular-targeting agent ZD6126. Clin Cancer Res. 2004;10(11):36503657.

25. Heethuis SE, van Rossum PSN, Lips IM, et al. Dynamic contrast-enhanced MRI for treatment response assessment in patients with oesophageal cancer receiving neoadjuvant chemoradiotherapy. Radiother Oncol. 2016;120(1):128-135.

26. Sclafani F, Smyth E, Cunningham D, Chau I, Turner A, Watkins D. A pilot study assessing the incidence and clinical significance of circulating tumor cells in esophagogastric cancers. Clin Colorectal Cancer. 2014;13(2):94-99.

27. Reeh M, Effenberger KE, Koenig AM, et al. Circulating Tumor Cells as a Biomarker for Preoperative Prognostic Staging in Patients With Esophageal Cancer. Ann Surg. 2015;261(6):1124-1130.

28. Gopalan V, Lam AK. Circulatory Tumor Cells in Esophageal Adenocarcinoma. Methods Mol Biol. 2018; 1756:177-186.

29. Creemers A, Krausz S, Strijker M, et al. Clinical value of ctDNA in upper-GI cancers: A systematic review and meta-analysis. Biochim Biophys Acta. 2017;1868(2):394-403.

30. Boellaard R, Delgado-Bolton R, Oyen WJG, et al. FDG PET/CT: EANM procedure guidelines for tumour imaging: version 2.0. Eur J Nucl Med Mol Imaging. 2015;42(2):328-354.

31. Corradetti M, Hatch J, Torok J, et al. Dynamic Changes in Cell-Free DNA During Chemoradiation for Non-small Cell Lung Cancer. Int J Radiat Oncol. 2017;99(2):S114-S115.

32. van Ginkel JH, van den Broek DA, van Kuik J, et al. Preanalytical blood sample workup for cell-free DNA analysis using Droplet Digital PCR for future molecular cancer diagnostics. Cancer Med. 2017;6(10):22972307.

33. National guideline esophageal cancer (version 3.1). 2015. http://www.oncoline.nl/oesofaguscarcinoom. Accessed March 21, 2018.

34. Washington K. 7th Edition of the AJCC Cancer Staging Manual: Stomach. Ann Surg Oncol. 2010;17(12):30773079.

35. Chirieac LR, Swisher SG, Ajani JA, et al. Posttherapy pathologic stage predicts survival in patients with esophageal carcinoma receiving preoperative chemoradiation. Cancer. 2005;103(7):1347-1355.

36. Mandard AM, Dalibard F, Mandard JC, et al. Pathologic assessment of tumor regression after preoperative chemoradiotherapy of esophageal carcinoma. Clinicopathologic correlations. Cancer. 1994;73(11):26802686.

37. Bossuyt PM, Reitsma JB, Bruns DE, et al. STARD 2015: an updated list of essential items for reporting diagnostic accuracy studies. BMJ. 2015;351:h5527.

38. Collins GS, Reitsma JB, Altman DG, Moons KGM. Transparent reporting of a multivariable prediction model for individual prognosis or diagnosis (TRIPOD): The TRIPOD Statement. BMJ. 2015;350:g7594.

39. Austin PC, Steyerberg EW. Events per variable (EPV) and the relative performance of different strategies for estimating the out-of-sample validity of logistic regression models. Stat Methods Med Res. 2017;26(2):796808.

40. Peduzzi P, Concato J, Kemper E, Holford T, Feinstein A. A simulation study of the number of events per variable in logistic regression analysis. J Clin Epidemiol. 1996;49(12):1373-1379.

41. Taketa T, Correa AM, Suzuki A, et al. Outcome of Trimodality-Eligible Esophagogastric Cancer Patients Who Declined Surgery after Preoperative Chemoradiation. Oncology. 2012;83(5):300-304.

42. Noordman BJ, Wijnhoven BPL, Lagarde SM, et al. Active surveillance in clinically complete responders after neoadjuvant chemoradiotherapy for esophageal or junctional cancer. Dis Esophagus. 2017;30(12):1-8.

43. Semenkovich TR, Meyers BF. Surveillance versus esophagectomy in esophageal cancer patients with a clinical complete response after induction chemoradiation. Ann Transl Med. 2018;6(4). 
PART II | Chapter 7

44. Wieder HA, Ott K, Lordick F, et al. Prediction of tumor response by FDG-PET: comparison of the accuracy of single and sequential studies in patients with adenocarcinomas of the esophagogastric junction. Eur J Nucl Med Mol Imaging. 2007;34(12):1925-1932.

45. van Rossum PSN, Fried D V, Zhang L, et al. The Incremental Value of Subjective and Quantitative Assessment of 18F-FDG PET for the Prediction of Pathologic Complete Response to Preoperative Chemoradiotherapy in Esophageal Cancer. J Nucl Med. 2016;57(5):691-700.

46. Noordman BJ, Wijnhoven BPL, Lagarde SM, et al. Neoadjuvant chemoradiotherapy plus surgery versus active surveillance for oesophageal cancer: a stepped-wedge cluster randomised trial. BMC Cancer. 2018;18(1):142. 


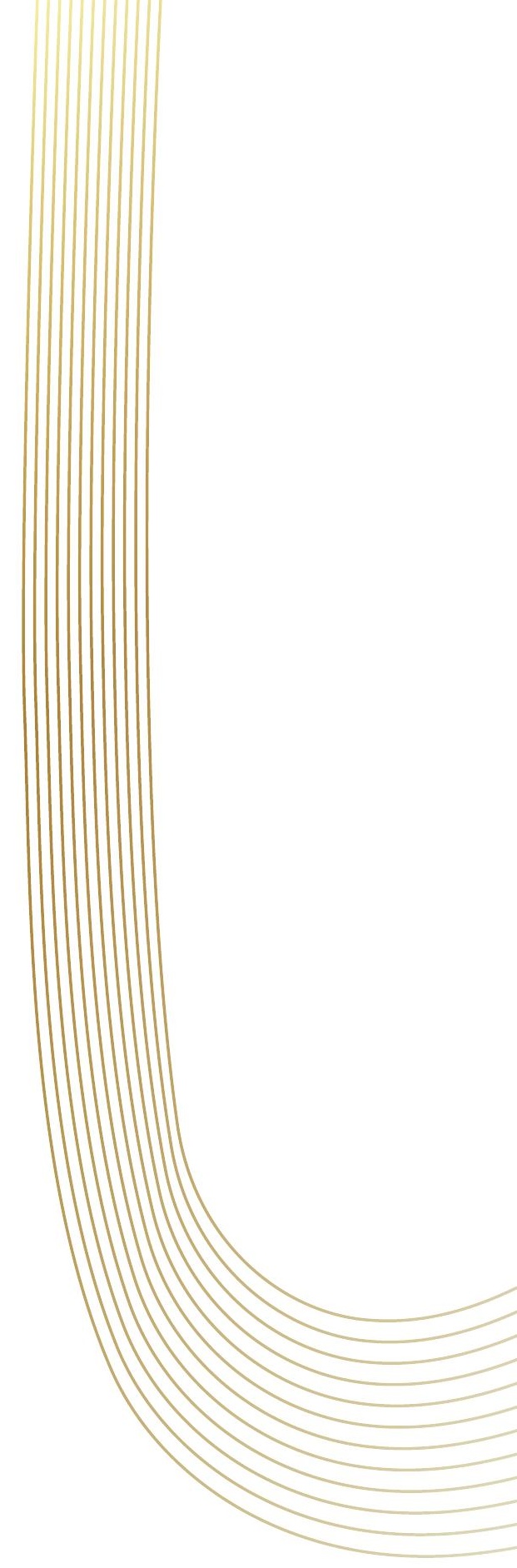


Chapter 8

\section{Patient perspectives on repeated MRI and PET/CT examinations during neoadjuvant treatment of esophageal cancer}

British Journal of Radiology 2018;91(1086):20170710.

L. Goense, A.S. Borggreve, S.E. Heethuis, A.L.H.M.W. van Lier, R. van Hillegersberg, S. Mook, G.J. Meijer, P.S.N. van Rossum, J.P. Ruurda 


\begin{abstract}

\section{Objective}

The perceived burden of diagnostic tests by patients during the assessment of esophageal cancer warrants attention with the current increase in repeated imaging for purposes of disease monitoring during and after treatment. The purpose of this prospective study was to evaluate the experienced burden associated with repeated MRI and PET/CT examinations during neoadjuvant treatment for esophageal cancer from the perspective of the patient.
\end{abstract}

\title{
Methods
}

In 27 patients receiving neoadjuvant chemoradiotherapy (nCRT) for esophageal cancer MRI and PET/CT examinations were performed before nCRT, during nCRT and before surgery. The experienced burden during repeated MRI and PET/CT examinations was evaluated with a self-report questionnaire addressing discomfort, pain, anxiety, and embarrassment, each measured on a 5 -point Likert scale ( $1=$ none; up to $5=$ very much). In addition, a comparative assessment was used to rank MRI, PET/CT and baseline endoscopy.

\section{Results}

All scans were performed without the occurrence of an adverse event. Few patients experienced discomfort (mean score \pm SD: $1.9 \pm 1.0$ for MRI versus $2.0 \pm 1.0$ for PET/CT, $p=0.586)$, pain (1.1 \pm 0.4 for MRI versus $1.3 \pm 0.7$ for PET/CT, $p=0.059)$, anxiety $(1.0 \pm 0.2$ for MRI versus $1.0 \pm 0.2$ for PET/CT, $p=1.000)$ and embarrassment ( $1.0 \pm 0$ for MRI versus $1.0 \pm 0.2$ for PET/CT, $p=0.317)$ during both MRI and PET/CT. Patients preferred MRI over PET/CT (67\% versus 22\%, respectively, $p=0.023$ ), and MRI over endoscopy (59\% versus $19 \%$, respectively, $p=0.027)$. In the comparison between PET/CT and endoscopy, 59\% of patients preferred PET/CT and 26\% preferred endoscopy $(p=0.093)$.

\section{Conclusion}

Repeated imaging with both MRI and PET/CT is generally well-tolerated for the assessment of response to treatment in esophageal cancer patients. Shorter acquisition times and altered body positioning during scanning will likely improve patient experience. 


\section{Introduction}

Esophageal cancer affects more than 450000 people yearly, and is the sixth most common cause of cancer-related deaths worldwide. ${ }^{1}$ Esophageal cancer is currently diagnosed by endoscopy with biopsy combined with multimodality imaging for staging. Since its clinical introduction, whole-body ${ }^{18} \mathrm{~F}$-fluorodeoxyglucose $\left({ }^{18} \mathrm{~F}-\mathrm{FDG}\right)$ positron emission tomography with integrated computed tomography (PET/CT) has become an important part of the standard work-up in esophageal cancer staging. ${ }^{2,3}$ Currently, high-resolution magnetic resonance imaging (MRI) is emerging as an advanced imaging technique for the staging of different types of cancer, including esophageal cancer. ${ }^{4-8}$

In general, diagnostic performance is the main motivation for implementing any diagnostic test, however, acceptability to patients is also an essential consideration. This is particularly relevant in esophageal cancer, given the increase in diagnostic testing for purposes of disease monitoring during and after treatment (e.g. response assessment, restaging, and recurrence detection). ${ }^{4,9,10}$ Patient experience is determined by several elements, including physical discomfort, the risk of side effects due to the investigation, anxiety, embarrassment and diagnostic accuracy. ${ }^{11-14}$

Although many reports are available that have assessed the diagnostic ability of different tests in the (re)staging of patients with esophageal cancer, little is known about the perceived burden of these tests by patients. In order to improve patient-friendliness of disease monitoring and (re)staging procedures, it is necessary to evaluate diagnostic procedures from the perspective of the patient. For this reason the imaging community recently emphasized that more research should be performed in this field. ${ }^{15}$ Therefore, the purpose of this prospective study was to assess the experienced burden for the patient associated with repeated MRI and PET/CT scanning during preoperative treatment for esophageal cancer, as determined by a questionnaire.

\section{Methods}

Data were collected in a prospective study evaluating the distinct and combined value of MRI (i.e. anatomical as well as functional diffusion-weighted and dynamic contrast enhanced MRI) and PET/CT to predict treatment response to neoadjuvant chemoradiotherapy (nCRT) in patients with esophageal cancer. This prospective study was approved by the institutional review board and written informed consent was provided by all patients. The study was registered with ClinicalTrials.gov, number NCT02125448. The current study concerns an ancillary study within the prospective study, evaluating the burden of additional MRI and PET/CT examinations using a questionnaire. 


\section{Study population}

Patients with newly diagnosed biopsy-proven esophageal cancer planned to receive neoadjuvant chemoradiotherapy according to the CROSS regimen ${ }^{16}$ followed by surgery were eligible for inclusion. Exclusion criteria included a history of thoracic radiotherapy and contraindications for MRI or PET/CT imaging (NCT02125448).

\section{Diagnostic procedures}

Initial diagnostic work-up consisted of endoscopy with biopsy, endoscopic ultrasound, cervical ultrasonography, and an integrated PET/CT for clinical staging. In addition to the initial clinical work-up, patients underwent additional MRI examinations before the start of nCRT, during nCRT after the first 9-15 days (median: 10 days) from the initiation of treatment, and 4-8 weeks (median: 7 weeks) after completion of nCRT but before surgery. Additional PET/CT examinations were performed during nCRT after the first 9-15 days (median: 10 days) from the initiation of treatment, and 4-8 weeks (median: 7 weeks) after completion of nCRT.

\section{Magnetic resonance imaging}

Patients underwent MRI scanning with anatomical (T2-weighted) and functional (diffusionweighted and dynamic contrast-enhanced) MRI sequences. The MRI examinations were performed on a 1.5 Tesla scanner equipped with a 16 or 28 -element phased-array receive coil for thoracic imaging (Achieva or Ingenia, Philips Medical Systems, Best, the Netherlands). The MRI scan protocol was specifically developed for esophageal cancer patients. ${ }^{2}$ Patients were scanned in supine position with arms parallel to the body without administration of anti-peristaltic agents. Sagittal and transverse T2-weighted images were obtained with a navigator that monitors the position of the diaphragm using a fast 1D-MRI acquisition, in order to trigger scanning exclusively during the end of the expiration. ${ }^{17} \mathrm{~A}$ dynamic contrast-enhanced MRI (DCE-MRI) series was obtained using the contrast agent gadobutrol (Gd-BT-DO3A, Gadovist; Schering AG, Berlin, Germany), injected at a dose of $0.1 \mathrm{mmol} / \mathrm{kg}$ of body weight with an automatic syringe pump at a flow rate of $1 \mathrm{~mL} / \mathrm{s}$ followed by saline injection. All MR images were obtained under free breathing conditions. All participants used the same earplugs (E.A.R. Soft FX; 3M BV) with a reported single number rating of $36 \mathrm{~dB}$, combined with headphones. Patients were allowed to select the music they listened to during the MRI examination. The MRI scanning time took approximately 40 minutes per examination.

\section{Positron emission tomography}

The ${ }^{18} \mathrm{~F}-\mathrm{FDG}$ PET scans were performed on a dedicated PET/CT system (mCT, Siemens, Erlangen, Germany). Patients underwent injection of ${ }^{18} \mathrm{~F}-\mathrm{FDG}$ after fasting for at least six 
hours. Before injection of FDG, blood glucose levels were checked in every patient to exclude hyperglycemia. The dose of intravenously administered ${ }^{18} \mathrm{~F}-\mathrm{FDG}$ ranged between 190-370 MBq. Imaging started 60-90 minutes after administration of ${ }^{18} \mathrm{~F}-\mathrm{FDG}$ with a CT for attenuation correction. The scanning time took approximately 30 minutes per examination. Following CT, PET scanning was performed from thigh to the base of skull in three-dimensional (3D) acquisition mode with 2-5 minutes per bed position. Patients were scanned in radiation treatment position (supine position with arms extended above the head).

\section{Data collection}

The experienced burden for the patient associated with the repeated MRI and PET/CT examinations during the clinical work-up was evaluated by means of a questionnaire at the end of the third MRI and PET/CT examination. A questionnaire was handed out after the final tests and patients were requested to complete the questionnaire at that moment. The questionnaire consisted of three modules. First, a standard formatted 5-point Likert scoring module ${ }^{18}$ was used to assess items concerning discomfort, pain, anxiety and embarrassment during the examinations that has previously been used in several other imaging studies (Table 2). ${ }^{11-14}$ Second, a comparative module was used, forcing patients to rank different tests (MRI vs. PET-CT vs. conventional staging [i.e. endoscopy]) from least to most inconvenient. Finally, a behavioral intent module was used by asking patients whether or not they, if opportune, would be willing to undergo the specific tests again from a scale of 1 (certainly not) to 5 (certainly yes). Patients were free to write additional comments for each module. The different modules were collated into one comprehensive questionnaire (see Online Supplement 1).

\section{Statistical analysis}

The responses of the patients to the different questions for MRI and PET/CT were described as counts with percentages, mean with standard deviation (SD) or median with range as appropriate. Likert scores for MRI and PET/CT were statistically compared using the Wilcoxon signed rank tests in case pairwise comparisons were applicable. For comparison of patient preferences the non-parametric sign test was used. A p-value of less than 0.05 was considered statistically significant. Statistical analyses were performed using SPSS 23.0 (IBM Corp, Armonk, NY, USA). 


\section{Results}

Between November 2013 and August 2015, a total of 32 consecutive patients with newly diagnosed esophageal cancer who underwent standard diagnostic work-up signed informed consent. Five patients were excluded from further analyses. Three patients withdrew from study participation, one had unexpected distant metastatic disease during nCRT and did not finish the study, and one did not return the questionnaire. The remaining 27 patients were eligible for further analysis. The study population had a mean age of 63.6 years (SD: 7.6 years), and 23 (85\%) of the patients were male. Histologic tumor types included adenocarcinoma $(n=21,78 \%)$, squamous cell carcinoma $(n=4,15 \%)$ or other types $(n=2$, $7 \%$ ). All scans of the 27 patients were performed without the occurrence of an adverse event.

The results of the questionnaires and the difference in MRI and PET/CT are partially demonstrated in Table 2 and Figure 1. Few patients experienced anxiety or embarrassment during MRI and PET/CT scanning, and no statistically significant difference between the tests was observed. With regard to pain, most patients described both MRI (mean: 1.1, SD: 0.4, range: $1-3$ ) and PET/CT (mean: 1.3 , SD: 0.7, range: $1-4$ ) as not or little painful, and no significant difference in pain was experienced between the two examinations $(p=0.059)$. In general, patients experienced little discomfort during both MRI (mean 1.9, SD: 1.0, range: 1-5) and PET/CT (mean: 2.0, SD: 1.0, range: 1-5) scanning, and no statistical difference in discomfort was observed between the two examinations $(p=0.586)$. However, a few patients reported high scores of discomfort during either MRI $(n=2)$ or PET/CT $(n=2)$. This was caused by either the position of the body during scanning (MRI $n=1$, PET/CT $n=2$ ) or the noise caused by the MRI scanner $(n=1)$.

When specifically asked what part of the procedure caused the most discomfort (Table 3 ), patients reported that the main cause of discomfort was the body position in the scanner during MRI $(n=6,22 \%)$ and PET/CT $(n=14,52 \%)$. The scanning time of both MRI $(22 \%)$ and PET/CT (15\%) was also considered a considerable burden. The necessary waiting time before PET/CT scanning was considered as a burden by 5 (19\%) of the 27 patients. The noise caused by the MRI scans was indicated to be unpleasant by 7 (26\%) of the patients.

Table 1. Characteristics of the three diagnostic tests

\begin{tabular}{llllll}
\hline Procedure & Fasting & IV line & Sedation & Pharmaceutical & Duration $(\mathbf{m i n})$ \\
\hline MRI & No & Yes & No & Gadobutrol (Gadovist) & $\approx 30$ \\
PET/CT & Yes & Yes & No & ${ }^{18}$ F-FDG & $\approx 90-120$ \\
& & & & & $\begin{array}{l}\text { (of which } 30 \text { minutes actual } \\
\text { scanning time) }\end{array}$ \\
Endoscopy & Yes & Yes $^{*}$ & Yes $^{*}$ & Midazolam & \\
\hline
\end{tabular}

$I V$ intravenous; * optional 
Table 2. Reported burden scores during MRI and PET/CT scanning for discomfort, pain, anxiety, embarrassment and repeated tests, as well as reported willingness to undergo similar tests in the future

\begin{tabular}{|c|c|c|c|c|c|c|c|}
\hline \multirow[t]{2}{*}{ Item } & \multicolumn{3}{|c|}{ MRI } & \multicolumn{3}{|c|}{$\mathrm{PET} / \mathrm{CT}$} & \multirow[b]{2}{*}{ p-value } \\
\hline & Mean $( \pm \mathrm{SD})$ & Median & Range & Mean $( \pm \mathrm{SD})$ & Median & Range & \\
\hline Discomfort * & $1.9(1.0)$ & 2 & $1-5$ & $2.0(1.0)$ & 2 & $1-5$ & 0.586 \\
\hline Pain * & $1.1(0.4)$ & 1 & $1-3$ & $1.3(0.7)$ & 1 & $1-4$ & 0.059 \\
\hline Anxiety* & $1.0(0.2)$ & 1 & $1-2$ & $1.0(0.2)$ & 1 & $1-2$ & 1.000 \\
\hline Embarrassment * & $1.0(0.0)$ & 1 & 1 & $1.0(0.2)$ & 1 & $1-2$ & 0.317 \\
\hline Additional burden of tests* & $1.3(0.5)$ & 1 & $1-3$ & $1.4(0.6)$ & 1 & $1-3$ & 0.132 \\
\hline $\begin{array}{l}\text { Willingness to undergo } \\
\text { similar tests in the future }\end{array}$ & $4.3(0.7)$ & 4 & $3-5$ & $4.2(0.8)$ & 4 & $2-5$ & 0.317 \\
\hline
\end{tabular}

*: $1=$ none; $2=$ little; $3=$ quite; $4=$ very; $5=$ very much

†: 1 = absolutely not; 2 = probably not; $3=$ neutral; $4=$ probably yes; $5=$ absolutely yes

Only a small number of patients considered the insertion of the intravenous line (MRI: $\mathrm{n}=1$, PET/CT: $\mathrm{n}=1$ ) or being in a small room during the tests (MRI: $\mathrm{n}=1$, PET/CT: $\mathrm{n}=1$ ) as a cause of discomfort. One patient noted that fasting for at least 6 hours prior to the PET/ CT examination had been very uncomfortable while losing weight due to disease and treatment burden. Another patient reported that it could get uncomfortably warm inside the MR scanner during the scanning.

In the comparative module patients were asked to rank different tests regarding comfort (Figure 2). Eighteen out of 27 patients (67\%) preferred MRI over PET/CT, 6 (22\%) preferred PET/CT over MRI, and $3(11 \%)$ did not express a preference $(p=0.023)$. In the comparison between PET/CT and endoscopy, 16 (59\%) patients preferred PET/CT, 7 (26\%) endoscopy and $4(15 \%)$ did not express a preference $(p=0.093)$. When asked to choose between MRI and endoscopy, 16 (59\%) patients preferred MRI, 5 (19\%) endoscopy and 6 (22\%) did not express a preference $(p=0.027)$.

Overall the additional burden of the MRI (mean: 1.3, SD: 0.5, range: 1-3) or PET/CT (mean: 1.4, SD: 0.6 , range 1-3) on top of treatment and other diagnostic procedures was regarded as being acceptable and comparable between the two modalities $(p=0.132)$. When patients were asked whether or not they, if opportune, would be willing to undergo the MRI or PET/ CT scans again, most answered 'probably yes' ([mean: 4.3, SD: 0.7, range: $3-5]$ versus [mean: 4.2 , SD: 0.8 , range: $2-5], p=0.317$, respectively). Some patients noted that it gave them a good feeling being able to contribute to scientific research $(n=2)$. 


\section{Perceived burden during MRI and PET/CT scanning}

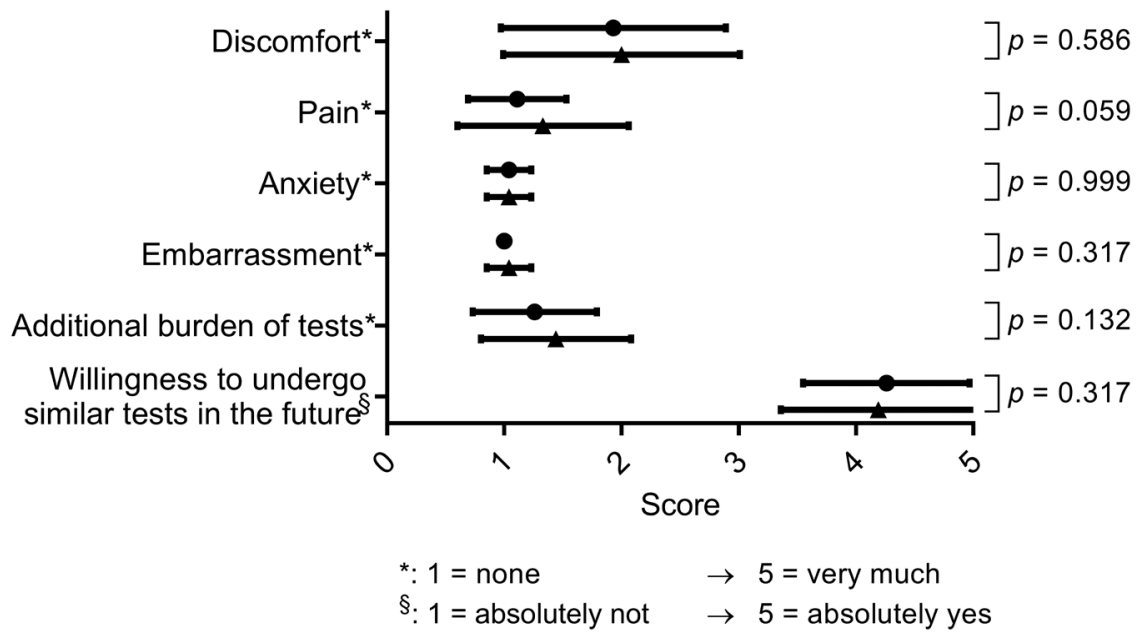

Figure 1. Perceived burden by the patients during MRI and PET/CT scanning

Table 3. Part of test procedure that caused most discomfort

\begin{tabular}{|c|c|c|c|c|}
\hline \multirow[t]{2}{*}{ Item } & \multicolumn{2}{|c|}{ MRI } & \multicolumn{2}{|c|}{ PET/CT } \\
\hline & $\mathbf{n}$ & $\%$ & $\mathbf{n}$ & $\%$ \\
\hline \multicolumn{5}{|l|}{ Most stressful part of test } \\
\hline Insertion of intravenous line & 1 & 4 & 1 & 4 \\
\hline Insertion of contrast (or FDG) & 0 & 0 & 0 & 0 \\
\hline Waiting time before scanning & NA & NA & 5 & 19 \\
\hline Scan time & 6 & 22 & 4 & 15 \\
\hline Body position in the scanner & 6 & 22 & 14 & 52 \\
\hline Noise of the scanner & 7 & 26 & 0 & 0 \\
\hline Being in a small room & 1 & 4 & 1 & 4 \\
\hline Non specifically & 6 & 22 & 2 & 7 \\
\hline
\end{tabular}

NA not applicable

\section{Discussion}

In this single center prospective study, patient experience of repeated MRI and PET/CT scanning during preoperative treatment for esophageal cancer was evaluated. The results of the current study show that patients experience MRI and PET/CT scans on top of treatment and other diagnostic examinations as a reasonable burden. When asked to rank the different tests, patients preferred MRI over PET/CT and endoscopy.

Several studies have shown that MRI is a feasible technique in esophageal cancer imaging, 


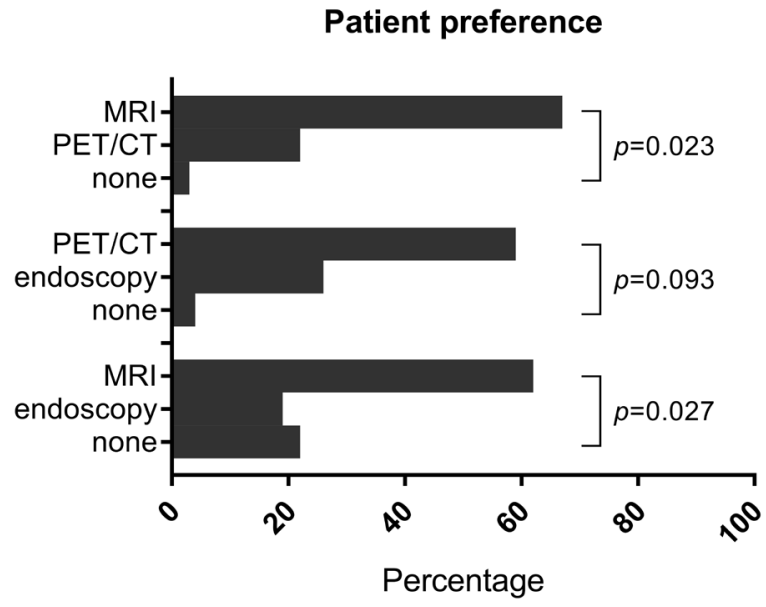

Figure 2. Comparative module forcing patients to rank the different tests from least to most inconvenient

and is a promising tool for response to treatment assessment ${ }^{4,6-8}$. Especially due to the unsatisfactory results in treatment response assessment of conventional diagnostic techniques (i.e. endoscopic ultrasonography ${ }^{19}$, endoscopy with biopsy ${ }^{19}$, and PET/CT ${ }^{20-22}$ ), the results of MRI for response assessment in esophageal cancer is currently of high clinical interest. From a patient perspective, the acceptable experience of MRI imaging in the current study supports its further utilization in clinical practice. Also, it is reassuring that patients find the additional burden of MRI and PET/CT examinations - on top of treatment and other diagnostic studies - acceptable, given the increase in repeated diagnostic examinations over the course of treatment and follow-up in esophageal cancer. ${ }^{2,45}$

Although the results of the current study indicate a slight overall patient preference of MRI over PET/CT, a large range of determinants influenced patient preferences with clear interindividual differences. The assumption, therefore, that MRI is better tolerated and preferred to PET/CT by all patients would not be valid. Patients place different personal weightings on the importance of test characteristics, which in turn influences overall test preference. However, the results of the current study do indicate actions that could improve patient experience during either MRI or PET/CT imaging. First, the fact that body position, the need to lie still, and duration of scanning were the main causes for discomfort during both MRI and PET/CT acquisition indicates that a more comfortable position and faster scanning may improve the acceptance of both MRI and PET/CT imaging. Second, the noise caused by the MRI scans was indicated to be unpleasant by 7 (26\%) of the 27 patients. Although earplugs combined with headphones with patient's preferred music were available, this was apparently not sufficient to reduce the noise to an acceptable level for all patients. 
The intrusive nature of the MRI scanner noise - despite measures to reduce the noise levels - has been previously noted by several studies assessing patient perception of MRI. ${ }^{23,24}$ To this regard, more noise reduction than provided in the current study should be made available, which may be a simple measure to improve patient perception. Third, careful patient preparation, including detailed verbal information and recognition for patients' emotions during the imaging exam, will likely improve patient compliance required for recording adequate images. ${ }^{25}$

Against the common expectation that the least invasive diagnostic test would have the patients' preference, 5 (19\%) and 7 (26\%) of the patients preferred endoscopy over MRI and PET/CT, respectively. These findings are most likely caused by the difference in administration of sedation and the timing of measurement of patient preferences between the examinations. In contrast to MRI and PET/CT, sedatives were administered to most patients during endoscopy which has shown to improve the acceptance of gastrointestinal endoscopy. ${ }^{26}$ Furthermore, the questionnaire administration was directly performed after the last MRI and PET/CT examination, while the last endoscopy was performed 10 to 15 weeks prior to the questionnaire. This difference in timing may have influenced patient experience of endoscopy over time. ${ }^{27}$ In future endeavors to compare patient preferences between endoscopy and imaging modalities, these factors should be taken into account in the study design.

Several limitations of the current study must be considered. First, patients did not receive information on the diagnostic performance of MRI and PET/CT for the assessment of response to treatment in esophageal cancer. Previous studies on patient perception of diagnostic modalities, however, have shown that diagnostic performance is a major aspect of overall patient preference. ${ }^{28}$ Second, it has been indicated that patients that volunteer to participate in prospective research are likely different from the initial target populations. ${ }^{29}$ In that regard, the burden of the additional scans may be underestimated by the current study, as patients who were informed about the study and found the additional scans too burdensome have probably refused to participate. Third, all MRI scans were acquired directly after PET/CT acquisition, which may have biased the results when considering the total duration of both procedures combined. Furthermore, MRI is a developing technique in the field of esophageal cancer imaging, with a multitude of available different acquisition protocols. Some of these protocols require intravenous contrast injection and they all have a specific sound level. Our results may therefore not be directly generalizable to other MRI acquisition techniques.

In conclusion, this study indicates that repeated imaging with both MRI and PET/CT is generally well-tolerated for the assessment of response to treatment in esophageal cancer patients. Shorter acquisition times and altered body positioning during scanning will likely improve patient experience during MRI and PET/CT acquisition. 


\section{References}

1. Pennathur A, Gibson MK, Jobe BA, et al. Oesophageal carcinoma. Lancet. 2013;381:400-12.

2. van Rossum PSN, van Lier ALHMW, Lips IM, et al. Imaging of oesophageal cancer with FDG-PET/CT and MRI. Clin Radiol. 2015;70:81-95.

3. Wong R, Walker-Dilks C, Raifu A. Evidence-based guideline recommendations on the use of positron emission tomography imaging in oesophageal cancer. Clin Oncol. 2012;24:86-104.

4. van Rossum PS, van Lier AL, van Vulpen M, et al. Diffusion-weighted magnetic resonance imaging for the prediction of pathologic response to neoadjuvant chemoradiotherapy in esophageal cancer. Radiother Oncol. 2015;115:163-170.

5. Heethuis SE, van Rossum PSN, Lips IM, et al. Dynamic contrast-enhanced MRI for treatment response assessment in patients with oesophageal cancer receiving neoadjuvant chemoradiotherapy. Radiother Oncol. 2016;120:128-135.

6. Sakurada A, Takahara T, Kwee TC, et al. Diagnostic performance of diffusion-weighted magnetic resonance imaging in esophageal cancer. Eur Radiol. 2009;19:1461-1469.

7. Kwee RM, Dik AK, Sosef MN, et al. Interobserver Reproducibility of Diffusion-Weighted MRI in Monitoring Tumor Response to Neoadjuvant Therapy in Esophageal Cancer. PLoS One. 2014;9:e92211.

8. Aoyagi T, Shuto K, Okazumi S, et al. Evaluation of the clinical staging of esophageal cancer by using diffusion-weighted imaging. Exp Ther Med. 2010;1:847-851.

9. Bruzzi JF, Swisher SG, Truong MT, et al. Detection of interval distant metastases: clinical utility of integrated CT-PET imaging in patients with esophageal carcinoma after neoadjuvant therapy. Cancer. 2007;109:12534.

10. Goense L, Van Rossum PSN, Reitsma JB, et al. Diagnostic performance of ${ }^{18}$ F-FDG PET and PET/CT for the detection of recurrent esophageal cancer after treatment with curative intent: A systematic review and meta-analysis. J Nucl Med. 2015;56:995-1002

11. Meléndez JC, McCrank E. Anxiety-related reactions associated with magnetic resonance imaging examinations. JAMA. 1993;270:745-7.

12. van Gelder RE, Birnie E, Florie J, et al. CT Colonography and Colonoscopy: Assessment of Patient Preference in a 5-week Follow-up Study. Radiology. 2004;233:328-337.

13. MacKenzie R, Sims C, Owens RG, et al. Patients' perceptions of magnetic resonance imaging. Clin Radiol. 1995;50:137-43.

14. Deutekom M, Terra MP, Dijkgraaf MGW, et al. Patients' perception of tests in the assessment of faecal incontinence. Br J Radiol. 2006;79:94-100.

15. Gunderman RB, Tillack AA. Empathy's Vital Role in Putting Patients First. Radiology. 2013;269:315-317.

16. van Hagen P, Hulshof MC, van Lanschot JJ, et al. Preoperative chemoradiotherapy for esophageal or junctional cancer. N Engl J Med. 2012;366:2074-2084.

17. Lever FM, Lips IM, Crijns SPM, et al. Quantification of Esophageal Tumor Motion on Cine-Magnetic Resonance Imaging. Int J Radiat Oncol. 2014;88:419-424.

18. Sullivan GM, Artino AR. Analyzing and Interpreting Data From Likert-Type Scales. J Grad Med Educ. 2013;5:541-542.

19. van Rossum PSN, Goense L, Meziani J, et al. Endoscopic biopsy and EUS for the detection of pathologic complete response after neoadjuvant chemoradiotherapy in esophageal cancer: a systematic review and meta-analysis. Gastrointest Endosc. 2016;83:866-79.

20. Chen Y, Pan X, Tong L, et al. Can ${ }^{18} \mathrm{~F}$-fluorodeoxyglucose positron emission tomography predict responses to neoadjuvant therapy in oesophageal cancer patients? A meta-analysis. Nucl Med Commun. 2011;32:1005-10.

21. Kwee RM. Prediction of tumor response to neoadjuvant therapy in patients with esophageal cancer with use of 18F FDG PET: a systematic review. Radiology. 2010;254:707-17.

22. van Rossum PSN, Fried D V, Zhang L, et al. The Incremental Value of Subjective and Quantitative Assessment of 18F-FDG PET for the Prediction of Pathologic Complete Response to Preoperative Chemoradiotherapy in Esophageal Cancer. J Nucl Med. 2016;57:691-700.

23. McJury M, Shellock FG. Auditory noise associated with MR procedures: a review. J Magn Reson Imaging. 2000;12:37-45. 
24. Hafeez R, Wagner C V, Smith S, et al. Patient experiences of MR colonography and colonoscopy: a qualitative study. Br J Radiol. 2012;85:765-9.

25. Youssefzadeh S, Eibenberger K, Helbich T, et al. Reduction of adverse events in MRI of the breast by personal patient care. Clin Radiol. 1997;52:862-4.

26. Cohen LB, Wecsler JS, Gaetano JN, et al. Endoscopic Sedation in the United States: Results from a Nationwide Survey. Am J Gastroenterol. 2006;101:967-974.

27. Brédart A, Razavi D, Robertson C, et al. Timing of patient satisfaction assessment: effect on questionnaire acceptability, completeness of data, reliability and variability of scores. Patient Educ Couns. 2002;46:131-6.

28. von Wagner C, Halligan S, Atkin WS, et al. Choosing between CT colonography and colonoscopy in the diagnostic context: a qualitative study of influences on patient preferences. Heal Expect. 2009;12:18-26.

29. Stuart EA, Bradshaw CP, Leaf PJ. Assessing the Generalizability of Randomized Trial Results to Target Populations. Prev Sci. 2015;16:475-485. 



\section{PART III}

Surgical treatment 


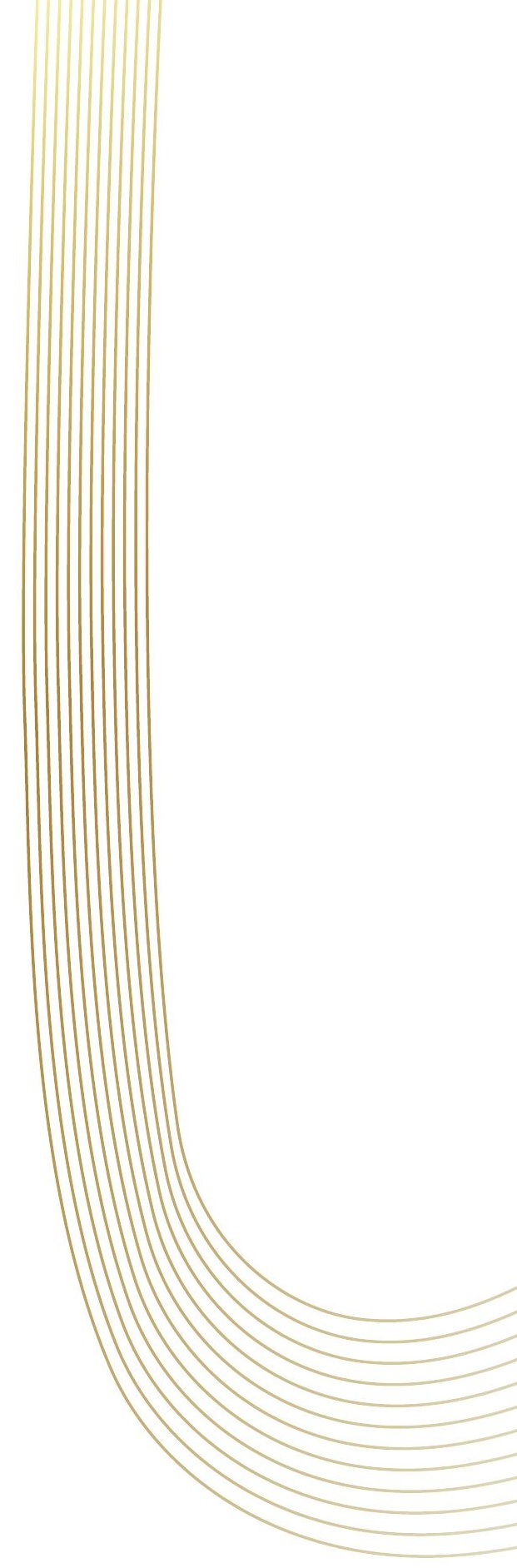


Chapter 9

\section{Surgical treatment in the era of multimodality management of esophageal cancer}

Annals of the New York Academy of Sciences 2018;1434(1):192-209.

A.S. Borggreve ${ }^{\star}$, B.F. Kingma ${ }^{\star}$, S.A. Domrachev, M.A. Koshkin, J.P. Ruurda, R. van Hillegersberg, F.R. Takeda, L. Goense

* Joint first authorship 


\begin{abstract}
Over the last decades, the treatment of resectable esophageal cancer has evolved into a multidisciplinary process in which all players are essential to be successful. Medical oncologists and radiation oncologists are increasingly involved since the implementation of neoadjuvant therapy, which has shown to improve survival. Although esophagectomy is still considered the cornerstone of curative treatment for locally advanced esophageal cancer, it remains associated with considerable postoperative morbidity, despite promising results of minimally invasive techniques. In this light, both physical status and response to neoadjuvant therapy may be important factors for selecting patients who will benefit from surgery. Furthermore, it is important to optimize the entire perioperative trajectory: from the initial outpatient clinic visit to postoperative discharge. Enhanced Recovery After Surgery (ERAS) is increasingly recognized for esophagectomy and emphasizes perioperative aspects such as nutrition, physiotherapy, and pain management. To date, several facets of esophageal cancer treatment remain topic of debate, such as the preferred neoadjuvant treatment, anastomotic technique, extent of lymphadenectomy, organization of postoperative care and the role of surgery beyond locally advanced disease. This review aims to describe the current and future perspectives in the surgical treatment of patients with esophageal cancer in the context of available literature.
\end{abstract}




\section{Introduction}

The management of esophageal cancer is complex, and highly variable between countries and centers. Multimodality treatment, which implies neoadjuvant chemoradiotherapy or perioperative chemotherapy in combination with esophageal resection, is increasingly applied over the world, since it has shown a survival benefit over surgery alone. ${ }^{1-4}$ Treatment teams nowadays not only consist of a surgeon, but are truly multidisciplinary with the participation of medical oncologists, radiation oncologists, gastroenterologists, dieticians and physiotherapists. Although the role of perioperative treatment has significantly evolved with the introduction of neoadjuvant and adjuvant treatment strategies, there is general international consensus that surgery remains a fundamental part of the curative treatment of locally advanced esophageal cancer. ${ }^{1}$

This review summarizes the advances in surgical treatment in the era of multimodality management of esophageal cancer. The first section gives an overview of patient selection for surgery and perioperative therapy. The second section addresses commonly used surgical techniques for esophageal cancer, with a particular focus on minimally invasive surgery, anastomotic techniques, the extent of lymphadenectomy, and the role of surgery in advanced disease. Finally, the introduction of enhanced recovery protocols after esophagectomy is discussed.

\section{Patient selection for surgery}

Esophagectomy is a highly complex surgical procedure that is associated with relatively high morbidity, mortality and recurrence rates. ${ }^{1}$ The eligibility of a patient for surgical resection strongly depends on the extent of the disease, as well as on the general condition of the patient. Decisions on the initial treatment approach of esophageal cancer are based on clinical staging, which should be carried out with the utmost accuracy. ${ }^{2}$ Adequate staging at least includes a complete clinical examination and a computed tomography (CT) scan of the neck, chest and abdomen. ${ }^{1,5}$ Furthermore, to determine whether lymphatic or distant metastatic disease is present in patients with more advanced tumors that are candidates for surgical resection, endoscopic ultrasound (EUS) and ${ }^{18} \mathrm{~F}$ fluorodeoxyglucose positron emission tomography-CT (PET-CT) should be performed. ${ }^{1,2,6,7}$ A multidisciplinary team is then necessary for choosing the appropriate treatment for each patient individually, not only based on the previously mentioned tumor stage, but also depending on tumor location, histological subtype, comorbidities and age. In general, patients with locally advanced disease (cT1N+ and cT2-4aN0-3), are potential candidates for neoadjuvant treatment followed by surgical resection. 
In patients with early esophageal cancer (high-grade dysplasia [Tis], mucosal [T1a], and submucosal cancer [T1b]), endoscopic therapy (endoscopic mucosal resection as well as endoscopic submucosal dissection) is highly successful and functions as the preferred therapeutic approach. ${ }^{2,8,9}$ Depending on several histopathological parameters of the resected specimen, such as irradicality, the presence of lymfovascular invasion, deeper submucosal tumor invasion or poor differentiation, subsequent surgical resection is indicated. ${ }^{1,10,11}$ In patients not eligible for surgery, endoscopic therapy followed by chemotherapy or chemoradiotherapy might be considered as alternative treatment.

In addition to tumor stage, the location of the esophageal tumor influences a patients' eligibility for surgical resection. Especially patients with proximal esophageal tumors are usually poor candidates for surgery because of limitations in surgical techniques (e.g., confined working space and poor overview). ${ }^{12}$ Furthermore, due to its anatomical proximity to the hypopharynx, surgery includes a combined pharyngo-laryngo-esophagectomy in some cases, resulting in permanent tracheostomy and impacting the quality of life of these patients enormously. ${ }^{13}$ Thus, these patients frequently depend on definitive chemoradiotherapy with encouraging 3 -year outcomes and acceptable toxicity. ${ }^{13}$

\section{Comorbidity}

Accurate patient selection for surgery does not only depend on tumor characteristics, but also on assessment of comorbidities, a patients' nutritional status and cardiopulmonary function.

Results of a large population based study showed that a substantial percentage of patients diagnosed with esophageal cancer has a history of hypertension (56\%), congestive heart failure (21\%), smoking (71\%), and diabetes mellitus (18\%). ${ }^{14}$ This is reflected in the majority of patients having an American Society of Anesthesiologists (ASA) score of 3 (65\%). ${ }^{14}$ From a cohort of 1057 patients that underwent transthoracic esophagectomy, only $31.6 \%(n=334)$ fulfilled the criteria of a low comorbidity status (ASA score $\leq 2$, WHO/ECOG score $\leq 1$, age $\leq 65$ years, body mass index (BMI) $\left.19-29 \mathrm{~kg} / \mathrm{m}^{2}\right) .{ }^{15}$

The previously mentioned comorbidities are well known risk factors for several postoperative complications. For example, anastomotic leakage is associated with pre-existent cardiovascular disease such as heart failure, coronary artery disease, peripheral vascular disease and hypertension. ${ }^{14}$ In the context of risk factor evaluation, a patients' vascular status can be objectively evaluated by arterial calcification on a computed tomography (CT) scan. Patients with arterial calcifications have a higher risk of developing anastomotic leakage and esophageal conduit necrosis after esophagectomy compared to patients without calcifications. ${ }^{16-19}$ Preoperative identification of these high-risk patients could aid in selecting patients that would potentially benefit from ischemic conditioning of the gastric conduit to reduce anastomotic leakage rates. ${ }^{20,21}$ 
Some studies suggest that diabetes mellitus is associated with impaired anastomotic healing and therewith increases the risk of anastomotic leakage after esophagectomy. However, this relation has not been consistently described in literature. A recent review and meta-analysis including 16 observational studies concluded that diabetes mellitus was significantly associated with an increased risk of anastomotic leakage after esophagectomy (OR: 1.63; 95\% CI: $1.25-2.12 ; \mathrm{p}<0.001) .{ }^{22}$ Interestingly, in subgroup analyses, this association was mainly found in surgical populations from Europe and America (OR: 1.42; 95\% CI: 1.22 1.65; $\mathrm{p}<0.001$ ) but not in Asian populations (OR: 2.27; 95\% CI: 0.86-6.05; $\mathrm{p}=0.1$ ). ${ }^{22}$ The authors hypothesized that the limited number of included studies - with only 1540 Asian patients - was more likely to result in a weakened persuasive power of their pooled estimates. $^{22}$

Preoperative pulmonary function (reflected in the forced expiratory volume for 1 second expressed as percentage of the forced vital capacity percent (FEV1\%) and peak expiratory flow) is also associated with major complications after esophagectomy. ${ }^{23,24}$ Pulmonary complications can be reduced by minimally invasive surgery, thoracic epidural analgesia and early enteral nutrition. ${ }^{25}$ Other strategies to improve preoperative condition of the patient include physical therapy to increase cardiorespiratory function. ${ }^{26,27}$ A systematic review including 12 studies evaluating the effects of preoperative exercise therapy on postoperative complication rate and length of hospital stay concluded that preoperative exercise therapy can be effective for reducing postoperative complication rates and length of hospital stay after cardiac or abdominal surgery. ${ }^{28}$ The effect of preoperative inspiratory muscle training on the incidence of postoperative pneumonia in patients undergoing esophagectomy was studied in a multicenter randomized controlled trial (the PREPARE study), of which the results will be published in the near future. ${ }^{29}$

As far as patients that are not surgical candidates are concerned, definitive chemoradiotherapy functions as a suitable alternative to surgical resection. ${ }^{1}$

\section{Age}

Age has historically functioned as a selection criterion for treatment and management in cancer patients. The association of age with severity of complications after esophagectomy is demonstrated by an adjusted OR of 1.02 per year increase in age (95\% CI: 1.00-1.04, $\mathrm{p}=0.014) .{ }^{23}$ However, age itself might not influence postoperative outcomes as much as assumed. Age $\geq 80$ years is currently related to a decreased likelihood of esophagectomy compared to definitive chemotherapy (OR: 0.79, 95\% CI 0.65-0.96, p<0.015), as well as a decreased likelihood of palliative therapy versus no treatment (OR: 0.73, 95\% CI 0.65-0.81, $\mathrm{p}<0.001)$ and a decreased likelihood of trimodality therapy compared to definitive chemoradiation (OR: $0.15,95 \%$ CI 0.12-0.18, p<0.001) using taking persons between 70 and 79 years of age as a reference category. ${ }^{30}$ In this same series, all therapies were associated 
with improved survival in older age. Thus, old age should not be the only reason to exclude patients from surgical treatment that might improve survival. ${ }^{31}$ Treatment decisions should be made individually and with appropriate caution in older patients. A preoperative geriatric assessment is advisable to obtain a holistic view of the patient, which can give insight in the risk of postoperative complications, such as delirium. ${ }^{32}$

\section{Perioperative treatment for locally advanced esophageal cancer}

For locally advanced disease (cT1N+ and cT2-4aN0-3), treatment practices vary around the globe. However, there is a worldwide consensus that surgery alone should no longer be the standard of care for treatment of these tumors. ${ }^{33}$ Over the last decades, numerous strategies have been evaluated to improve the treatment results by adding perioperative or neoadjuvant chemo(radio)therapy to surgical resection. The MAGIC study ${ }^{4}$ and French FNCLCC/FFCD 9703 study $^{34}$ were the first to show an overall survival benefit for perioperative chemotherapy in patients with lower esophageal, GEJ and gastric adenocarcinoma. After these results, the CROSS study combined carboplatin/paclitaxel with 41.4 Gy radiation and demonstrated a $14 \%$ increase in 5 year overall survival for patients with esophageal cancer (both squamous cell carcinoma and adenocarcinoma) treated with neoadjuvant chemoradiotherapy followed by surgery compared to surgery alone. ${ }^{3,35}$ However, the recently presented phase III FLOT 4 trial has shown that the FLOT regimen (docetaxel, oxaliplatin, leucovorin, and 5-FU) improves the outcome of patients with esophageal adenocarcinoma and locoregional disease as compared to the ECF/ECX triplet (epirubicin, cisplatin, and 5-FU or capecitabine) ${ }^{36}$ Currently, several randomized trials comparing perioperative chemotherapy and neoadjuvant chemoradiotherapy are being undertaken. The NeoAegis study ${ }^{37}$ compares CROSS versus MAGIC and the German ESOPEC study compares FLOT versus CROSS in patients with esophageal adenocarcinoma ${ }^{38}$. The Chinese CMISG1701 study compares overall survival between neoadjuvant chemotherapy (cisplatin/paclitaxel) with neoadjuvant chemoradiotherapy (cisplatin/ paclitaxel with 40 Gy radiation), both followed by esophagectomy for locally advanced resectable esophageal squamous cell carcinoma. ${ }^{39}$ The NEOSCOPE study investigates whether induction chemotherapy followed by either oxaliplatin/capecitabine or paclitaxel/ carboplatin, both combined with 45 Gy radiation, is superior as pre-operative regimen in pathological complete response of resectable esophageal adenocarcinoma. ${ }^{40}$ Lastly, the PROTECT-1402 study compares 3 cycles of FOLFOX combined with concurrent radiotherapy $(41.4 \mathrm{~Gy})$ or carboplatin and paclitaxel with the same radiation regimen for esophageal and junctional cancer. ${ }^{41}$ An overview of these studies (including ClinicalTrials. gov identifiers) is presented in Table 1. 
Besides chemotherapy and radiation treatment, targeted therapies are emerging. Patients with esophageal adenocarcinoma should be tested for overexpression of the human epidermal growth factor receptor 2 (HER2) protein on the tumor biopsy. ${ }^{1}$ If a high level of HER2 expression is identified, HER2 antibodies such as trastuzumab and pertuzumab could be added to perioperative or neoadjuvant therapy (RTOG 1010 phase III study NCT01196390, PETRARCA phase II/III study NCT02581462, INNOVATION phase II study NCT02205047, TRAP phase Ib study NCT02120911). ${ }^{33}$ Another targeted therapy includes the addition of a vascular endothelial growth factor receptor (VEGFR)-2 antibody, ramucirumab, that interacts with the angiogenic pathway in tumorgenesis. ${ }^{33}$ Its value is currently assessed in patients with HER2-negative GEJ and gastric adenocarcinoma (RAMSES phase II/III study NCT02661971). Moreover, the addition of immunotherapeutic strategies to neoadjuvant chemoradiation or perioperative chemotherapy is currently evaluated, such as programmed death ligand 1 (PD-L1) targeted therapy with atezolizumab or pembrolizumab (PERFECT phase II study NCT03087864, KEYNOTE-590 phase III study NCT03189719).

From the adjuvant perspective, chemotherapy and radiotherapy have not been integral parts of the treatment of resectable esophageal cancer. A recent network meta-analysis demonstrated that, in contrast to surgeryi combined with neoadjuvant therapy, surgery combined with adjuvant therapies appeared lesser effective and showed no survival advantage when compared with surgery alone (HR: 0.87, 95\% CI 0.67-1.14, p=0.321). ${ }^{42}$ Furthermore, adjuvant chemotherapy after neoadjuvant chemoradiotherapy followed by surgery did not provide additional benefit for esophageal cancer patients without residual disease (ypT0N0, HR: 0.82, 95\% CI 0.50-1.35) or without non-nodal residual disease (ypT+N0, HR: 0.93, 95\% CI 0.74-1.17). ${ }^{43}$ Yet, in patients with residual nodal disease (ypTanyN+), adjuvant chemotherapy may provide a survival benefit (HR: 0.70, 95\% CI 0.57-0.85). ${ }^{43}$ However, since this has not been investigated in a formal randomized controlled trial, further research is needed before routine application in clinical practice.

\section{Complete responders to neoadjuvant therapy}

As a pathologic complete response to neoadjuvant therapy is observed in a substantial part of patients (up to $23 \%$ of patients with an adenocarcinoma and $49 \%$ of patients with a squamous cell carcinoma following neoadjuvant chemoradiotherapy ${ }^{3}$ ), it is argued that these patients may not benefit from subsequent surgery. ${ }^{44}$ Patients with a pathologic complete response have a favorable prognosis with a lower risk of developing a local regional recurrence and an increased survival compared to other subgroups. ${ }^{35,45-48}$ Several studies have been initiated to preoperatively identify these patients for an organ-preserving approach, avoiding unnecessary surgery related morbidity, followed by active surveillance. 


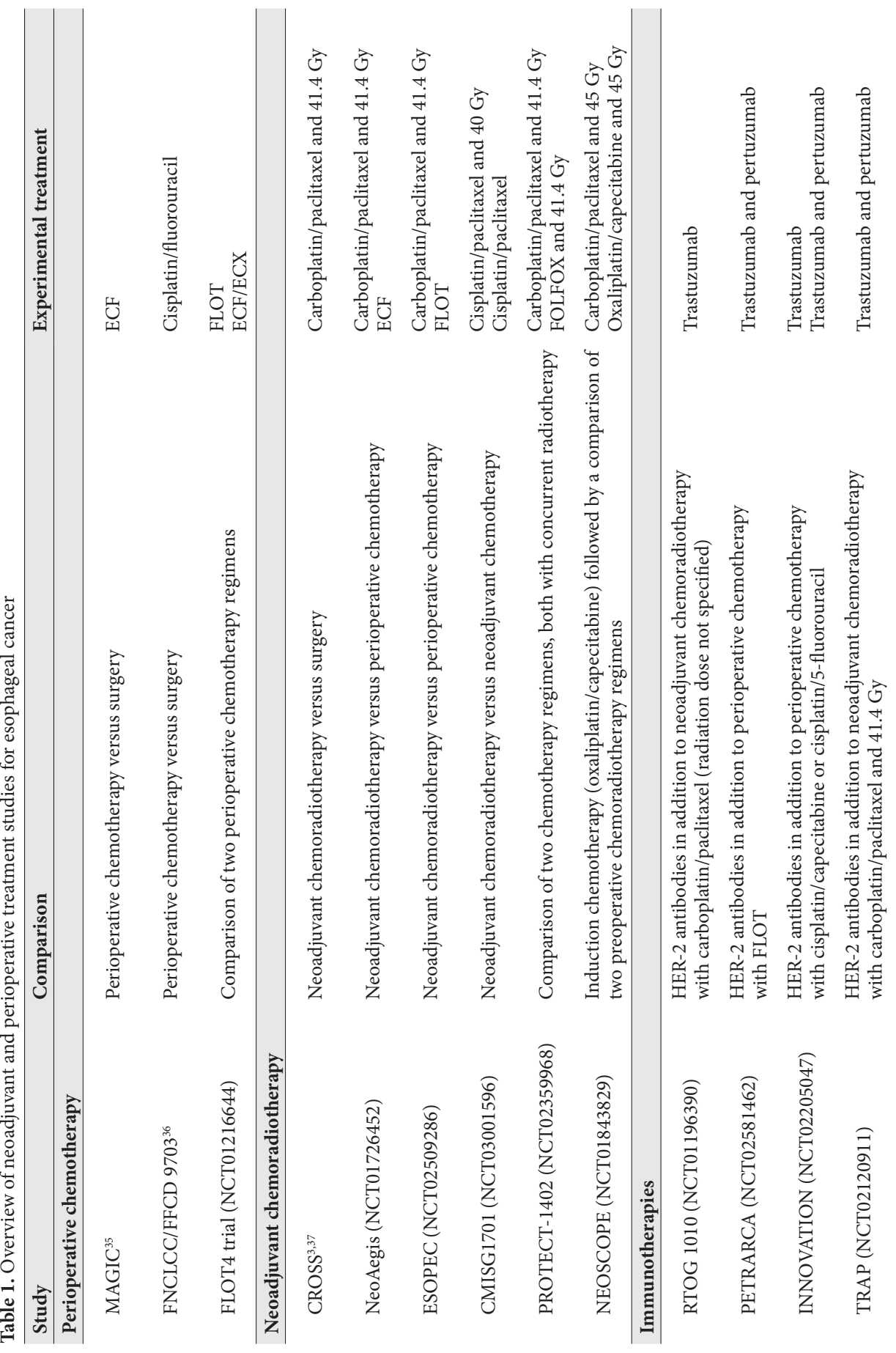




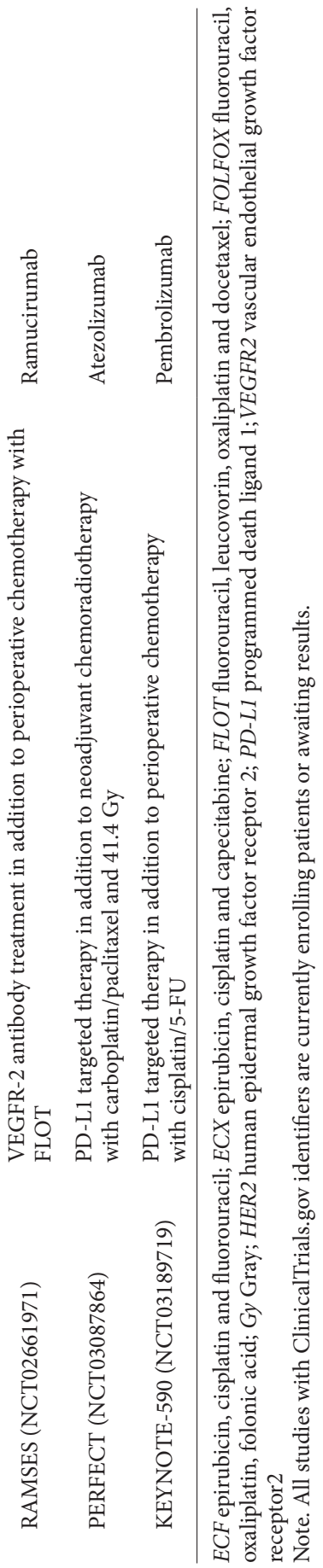


In this light, various diagnostic strategies such as endoscopic biopsies and/or EUS, ${ }^{49}$ 18F-FDG PET(-CT), ${ }^{50,51}$ diffusion weighted (DW) $)^{52-55}$ and dynamic contrast enhanced $(\mathrm{DCE})^{56}$ magnetic resonance imaging (MRI) are being researched to predict response and ultimately avoid surgery in selected patients. The MUNICON $-1^{57}$ and MUNICON-2 trials ${ }^{58}$ have shown that PET-based therapy individualization could be a feasible approach for GEJ adenocarcinomas. Furthermore, at least four large trials (recruiting $\geq 200$ patients) are currently studying the accuracy of various diagnostic modalities for pathologic complete response assessment and/or the safety of active surveillance after a complete clinical response (the French ESOSTRATE trial [NCT02551458], the Korean ESOPRESSO trial [NCT01740375], the Dutch PRIDE study and the Dutch SANO trial [Netherlands Trial Register ID NTR6803]).

\section{Enhanced Recovery After Esophagectomy in the preoperative setting}

The principles of Enhanced Recovery After Surgery (ERAS) were developed in 2001 by a group of surgeons who later established the ERAS Society. ${ }^{59}$ Since then, the ERAS Society has published various evidence-based multidisciplinary guidelines that aim to minimize the surgical stress response (minimally invasive techniques, carbohydrate loading), facilitate early postoperative mobilization (minimum number of drains, regional analgesia), and stimulate early postoperative resumption of oral intake. Although ERAS guidelines were published for several gastro-intestinal surgical procedures including colonic surgery, pancreaticoduodenectomy, rectal surgery, bariatric surgery, and gastrectomy, consensus is lacking regarding the optimal approach to patients undergoing esophagectomy. ${ }^{60-65}$ While the composition of the optimal perioperative care pathway remains subject of debate for

Table 2. Essential elements of Enhanced Recovery After Esophagectomy

\begin{tabular}{ll}
\hline At the outpatient clinic & $\begin{array}{l}\text { Structured multidisciplinary counseling } \\
\text { Evaluation and optimization of the nutritional state } \\
\text { Stimulation of smoking and alcohol cessation } \\
\text { Possibly inspiratory muscle training }\end{array}$ \\
Shortly before surgery & $\begin{array}{l}\text { Avoidance of prolonged fasting } \\
\text { Carbohydrate loading }\end{array}$ \\
During surgery & Minimally invasive surgical techniques \\
After surgery & $\begin{array}{l}\text { Stimulation of early postoperative mobilization } \\
\text { Adequate pain management }\end{array}$ \\
& $\begin{array}{l}\text { Limitation of drains, lines, and catheters } \\
\text { Possibly early resumption of oral intake }\end{array}$ \\
\hline
\end{tabular}


esophagectomy, several enhanced recovery protocols have been suggested with some reporting a median length of hospital stay of 8 days. ${ }^{66,67}$ Key elements of an Enhanced Recovery After Esophagectomy program can be found in Table 2.

\section{Outpatient clinic}

In the pre-admission phase, structured counseling by a multidisciplinary team is considered key to preparing patients for surgery. First, this increases information retention and offers the possibility correct potential misconceptions about disease and treatment, which can reduce patient anxiety ${ }^{68,69}$ Second, the time between diagnosis and surgery can be used to evaluate and monitor the nutritional state, which is at risk of deteriorating in esophageal cancer patients. At time of diagnosis, most esophageal cancer patients are malnourished due to local tumor effects causing dysphagia, anorexia, and cancer related cachexia. ${ }^{70}$ Weight loss and nutritional deficiencies are further increased by toxicities related to neoadjuvant therapy. Since malnutrition is in turn associated with an increased risk of postoperative infectious complications, nutritional screening is advised in all patients prior to esophagectomy. ${ }^{66,71-76}$

According to the latest guidelines of the European Society for Clinical Nutrition and Metabolism (ESPEN), nutritional intervention is indicated for patients who are at severe nutritional risk (i.e., $>10-15 \%$ weight loss within 6 months, BMI $<18.5 \mathrm{~kg} / \mathrm{m}^{2}$, Subjective Global Assessment Grade C, or serum albumin $<30 \mathrm{~g} / \mathrm{L}$ without evidence of hepatic or renal dysfunction). ${ }^{77,78}$ Nutritional intervention should involve nutrition support with sip feeds, enteral tube feeding, or parenteral feeding, in that order of preference. ${ }^{77,78}$

Various methods for nutritional intervention during neoadjuvant therapy prior to esophagectomy are summarized in a recently published review. ${ }^{70}$ All included studies showed that esophageal stents can be successfully used to palliate dysphagia, although the overall stent migration rate in these patients is $29.9 \% .{ }^{70}$ Instead of stenting to allow oral intake, enteral feeding can be initiated through a nasoduodenal, nasojejunal, or jejunostomy tube. Since long periods of nasoduodenal or nasojejunal tube feeding can be discomforting for patients, some surgeons advocate laparoscopic placement of a feeding jejunostomy at an early stage prior to esophagectomy. ${ }^{79}$ Studies on laparoscopically inserted jejunostomies were all of retrospective nature, and demonstrated an increase in weight ranging from 0.4 to $11.8 \mathrm{~kg} .{ }^{70}$ Only one comparative study was included in the previously mentioned review that compared esophageal stenting to jejunostomy feeding, which reported no significant difference between the two groups with respect to complication rates ( $22 \%$ for esophageal stenting versus $14 \%$ for jejunostomy, $\mathrm{p}=0.11$ ) or weight gain ( $4.4 \mathrm{~kg}$ for esophageal stenting versus $4.2 \mathrm{~kg}$ for jejunostomy, $\mathrm{p}=0.59) .^{70}$ The optimal nutritional approach for patients with resectable esophageal cancer undergoing neoadjuvant treatment prior to esophagectomy has thus to be found. 
Lastly, if applicable, patients should be encouraged and supported to cease alcohol abuse and smoking during the pre-admission phase, which reduces the risk of postoperative complications..$^{80,81}$

\section{Before surgery}

Shortly before esophagectomy, ERAS aims to minimize the surgical stress response and thereby decrease insulin resistance, which may reduce postoperative morbidity and length of hospital stay. ${ }^{82-84}$ In this context, ingestion of an oral carbohydrate solution is advised prior to esophagectomy as it is believed to cause an initial increase in baseline insulin sensitivity and in that way dampen surgery-induced insulin resistance. ${ }^{84}$ Although randomized controlled trials were inconsistent, a recent meta-analysis of studies including a variety of surgical procedures indicated that carbohydrate loading may be associated with a reduced length of hospital stay when compared to placebo or fasting. ${ }^{85,86}$ While the reported carbohydrate doses are varying, an amount of $400 \mathrm{~mL} 12.5 \%$ carbohydrate solution may be advised. ${ }^{78}$ With regard to the latter, it must be noted that prolonged preoperative fasting should be avoided to reduce patient discomfort and prevent the body from entering a catabolic state that aggravates the surgical stress response. ${ }^{87,88}$ The traditional practice of fasting from midnight before surgery was based on the doctrine that this would reduce the risk of aspiration during the induction anesthesia, which was based on old and sparse literature. ${ }^{82,89,90}$ Convincing evidence has emerged that ingestion of solid foods until 6 hours and clear liquids until 2 hours before elective surgery is safe, which led to adaptation of the ASA guidelines. ${ }^{91,92}$ These eased limitations can most likely also be applied to esophagectomy, although cautiousness is warranted in patients with dysphagia as they may have an increased risk of aspiration. ${ }^{66,78}$

\section{Minimally invasive surgery}

Traditional open esophagectomy (OE) is a highly invasive procedure that is associated with considerable morbidity. ${ }^{93-95}$ Over the last decades, minimally invasive techniques are increasingly adopted for esophageal surgery. ${ }^{96}$ An important aim of minimally invasive surgery is to reduce the physiological stress response to trauma, which can in turn diminish postoperative immunosuppression and susceptibility to infections. ${ }^{97,98}$ Additionally, laparoscopic and thoracoscopic procedures are generally associated with less pain when compared to their open alternatives, which promotes recovery. ${ }^{99-101}$ In this context, minimally invasive esophagectomy (MIE) was introduced in the 1990's and found to be a safe alternative to OE in various studies. ${ }^{102,103}$ The term MIE is used to describe an esophagectomy procedure that avoids the invasiveness of thoracotomy and laparotomy by 
performing thoracoscopy and laparoscopy, respectively. Although the worldwide implementation of MIE is at an advanced stage, only one randomized controlled trial was conducted to compare MIE to OE so far (i.e., the TIME trial). ${ }^{96,104}$ Patients were included from 5 high-volume centers (>30 esophagectomies per year) and MIE was significantly associated with fewer postoperative pulmonary infections during hospitalization (12\% versus 34\%) and within 2 weeks after surgery (9\% versus 29\%), less blood loss (median 200 versus $475 \mathrm{~mL}$ ), shorter hospitalization (median 11 versus 14 days), lower pain scores during the first 10 postoperative days (median Visual Analogue Scale 2 versus 3), and better shortterm quality of life scores when compared to OE. ${ }^{104}$ As no differences were found regarding 3 -year overall and disease-free survival, the results from this study suggest that MIE provides short-term advantages while maintaining oncological standards. ${ }^{104,105}$

Despite promising results for MIE, recent population-based studies did not unequivocally support its superiority over OE. ${ }^{106-109}$ While the length of hospitalization was repeatedly shown to be shorter for MIE, pulmonary complications and overall morbidity were generally equal. ${ }^{106-109}$ Moreover, a higher re-operation rate was consistently reported for MIE in these studies. ${ }^{106-109}$ The authors postulated that this might be attributable to a learning effect, as MIE is a relatively novel and technically complex procedure that is still in its implementation phase. ${ }^{107} \mathrm{~A}$ recent retrospective multicenter study investigated the learning curve for MIE and found that a plateau is reached after 119 cases, based on the evaluation of anastomotic leakage rates when making a transition from a cervical to an intrathoracic anastomosis. ${ }^{110}$ As the mean anastomotic leakage rate decreased from $18.8 \%$ (first time quintile) to $4.5 \%$ (fifth time quintile) during this time period, it appears that patients are exposed to additional risks during the learning phase. ${ }^{110}$

The thoracoscopic part of MIE is particularly difficult to master, especially when performing an intrathoracic anastomosis. A hybrid procedure, which avoids the need for a technically challenging thoracoscopic phase, has been proposed and investigated in a multicenter trial (i.e., the MIRO trial). ${ }^{111}$ Patients were randomly allocated to receive either hybrid (laparoscopy and thoracotomy) or open (laparotomy and thoracotomy) esophagectomy with an intrathoracic anastomosis. ${ }^{111}$ Although the full results were not made available yet, major pulmonary complications were reported to be significantly less frequent in the hybrid group $(30.1 \%$ versus $17.7 \%)$ in a recently published abstract by the American Society of Clinical Oncology. ${ }^{112}$ In combination with several case-matched studies, these findings suggest that morbidity may already be reduced by only performing the abdominal phase minimally invasive. ${ }^{113,114}$ In this light, hybrid esophagectomy might be an option for surgeons in low-volume centers that would face challenges in completing their thoracoscopic learning phase due to insufficient caseload. However, the additional value of avoiding an open thoracic phase has not yet properly been investigated and therefore comparative studies for full versus hybrid MIE are warranted. A pilot trial was conducted to investigate the 
feasibility of a randomized three-armed study that compares open versus hybrid versus full MIE. ${ }^{115}$ As patient recruitment was achieved rapidly, this trial is currently continued into a definitive randomized controlled trial.

In attempt to overcome the technical challenges of MIE, robot-assisted minimally invasive esophagectomy (RAMIE) was introduced in 2003. ${ }^{116}$ Robotic assistance allows for more natural hand movements and provides an enhanced three-dimensional vision of the surgical field. ${ }^{117}$ These adjustments increase accuracy and enable surgeons to perform more complex dissections, such as a meticulous mediastinal lymphadenectomy, or a hand-sewn intrathoracic anastomosis in the context of esophagectomy. Since its introduction, RAMIE has been shown to be a safe and oncologically adequate alternative to OE and conventional MIE. ${ }^{116-118}$ Furthermore, with its technical advantages, RAMIE may be an option for patients who were traditionally deemed inoperable due to a tumor or lymph node metastasis in the upper mediastinum. In a recent prospective study, 31 patients with an upper mediastinal tumor (cranial border at $18-24 \mathrm{~cm}$ from the incisors) or lymph node metastasis (level 2 and/or 4) underwent RAMIE and the authors concluded that the technique is feasible for this patient group, although it was associated with relatively high mortality (i.e., 10\%). ${ }^{119}$ More research is needed regarding the clinical merits and possibly extended operability criteria of RAMIE. The ROBOT-trial, which compared RAMIE to OE in terms of shortterm surgical and oncological outcomes, will be published soon. ${ }^{120}$

\section{Anastomotic techniques}

After esophagectomy for cancer, the gastrointestinal continuity is most commonly restored by gastric tube reconstruction with an esophagogastric anastomosis. Several anastomotic techniques are available to construct an esophagogastrostomy. In this section, postoperative morbidity and mortality, functional results, and oncologic outcomes of various commonly used anastomotic techniques are described.

\section{Cervical versus intrathoracic anastomosis}

During esophagectomy for cancer either the lowest part of the thoracic esophagus is resected followed by an intrathoracic esophagogastric anastomosis high in the chest, or the whole thoracic esophagus is resected combined with a part of the cervical esophagus followed by a cervical esophagogastric anastomosis. Both intrathoracic and cervical anastomoses are commonly performed worldwide, although the first is increasingly applied..$^{96}$

So far, 4 randomized trials have assessed clinical outcomes after either an intrathoracic or cervical anastomosis. ${ }^{121-124}$ In one of these clinical trials, the intrathoracic anastomosis was 
significantly associated with a lower anastomotic leakage rate when compared to the cervical anastomosis. ${ }^{121}$ Although the other three clinical trials did not report a statistical significant difference in leakage rates between the two methods ${ }^{122-124}$, two of these suggested a nonsignificant difference in leakage rates in favor of an intrathoracic anastomosis. ${ }^{123,124}$ This difference was confirmed by two meta-analyses - including all four clinical trials - that concluded that an intrathoracic anastomosis could be associated with lower anastomotic leakage rates (pooled leakage risk: $13.6 \%$ versus $2.9 \%{ }^{125},{ }^{126}$ for the cervical versus intrathoracic anastomosis, respectively). However, the evidence of the currently available clinical trials is limited because they all included a limited number of patients (32-92 patients per study) with few events (2-13 events per study), leading to uncertain estimates. ${ }^{121,124,127,128}$ In this regard, three large population based studies - including $2086^{129}$, $2944^{130}$ and $2969^{14}$ patients - confirmed that an intrathoracic anastomosis is associated with lower leakage rates and shorter hospital stay. Reported factors that may explain the lower leakage rate associated with an intrathoracic anastomosis include less tissue ischemia due to the shorter distance the blood supply must travel and less risk of tension on the anastomosis. ${ }^{14,126}$

Although leakage rates from the intrathoracic anastomosis may be lower, some surgeons prefer an cervical anastomosis because the sequelae of cervical anastomotic leakage are claimed to be less severe. ${ }^{131}$ It is hypothesized that cervical anastomotic leakage can be easily diverted by opening the neck wound and therewith prevent mediastinal contamination. However, the studies that support this hypothesis are rare, and included a limited number of patients. ${ }^{132,133}$ Furthermore, it should not be underestimated that cervical anastomotic leakage can also lead to severe intrathoracic complications (pleural empyema and mediastinal abscess) occurring in up to $62 \%$ of patients. ${ }^{134,135}$ To this regard, a previously mentioned meta-analysis and two large population based studies found no difference regarding in-hospital mortality between cervical and intrathoracic anastomoses. ${ }^{126,129,136}$ However, one of these studies indicated that length of intensive care stay and length of hospital stay was longer in patients after an intrathoracic anastomotic leakage. ${ }^{129}$ This suggests that the clinical course of anastomotic leakage is indeed more severe for intrathoracic anastomoses, but that this does not increase the risk of postoperative mortality.

Prevention of adverse events (i.e., recurrent laryngeal nerve palsy and stricture formation) leading to subsequent functional complaints (i.e., impaired swallowing and dysphagia) after esophagectomy is important because it can impair quality of life. In this context, a meta-analysis of clinical trials and a large population-based study assessed recurrent laryngeal nerve palsy rates after esophagectomy, and found that an intrathoracic anastomosis is associated with a statistically significant lower incidence of recurrent nerve trauma. ${ }^{126}$ This is likely explained by the close anatomical relation of the recurrent laryngeal nerve 
with the esophagus in the cervical region. Regarding benign strictures requiring dilatation, none of the 4 conducted clinical trials and the two subsequent meta-analysis reported a difference in the incidence between the cervical and intrathoracic anastomotic approach. ${ }^{121-126}$ It should be noted, however, that fair comparison of trials is restricted due to the lack of consensus regarding definition, diagnostic criteria, and threshold of treatment for anastomotic strictures.

Oncologic outcomes after esophageal surgery may also be influenced by the choice of anatomic location of the anastomosis, as a cervical anastomosis allows for a higher proximal resection margin. In this regard, one trial reported a statistically significantly higher irradical resection rate in in patients after an intrathoracic anastomosis compared to those receiving a cervical anastomosis (33\% versus $10 \%$, respectively; $\mathrm{p}<.05) .{ }^{124}$ Two other trials and a large population based study that also reported on this finding did not find a difference in radical resection rates. ${ }^{121,122,129}$ Patient selection remains important in this context, as patients with supracarinal tumors are likely not eligible for an intrathoracic anastomosis. However, owing to neoadjuvant chemoradiotherapy, an intrathoracic anastomosis is likely safe in patients with infracarinal tumors. ${ }^{137}$

In summary, based on the current available evidence an intrathoracic anastomosis is associated with reduced anastomotic leakage rates and recurrent nerve paresis rates compared to a cervical anastomosis. However, the currently available clinical studies are limited by their moderate quality and relatively small sample size. Currently a randomized controlled trial is underway in order to demonstrate whether a minimally invasive cervical or intrathoracic anastomosis should be preferred for patients with infracarinal esophageal tumors (the ICAN trial ${ }^{138}$ ).

\section{Hand-sewn versus stapled anastomosis}

Hand-sewn anastomosis and stapled anastomosis with a mechanical device are the most commonly used methods to construct an esophagogastric anastomosis. At present, the choice of a hand-sewn or stapled anastomosis mostly depends on the preference of the surgeon. So far, 10 clinical trials have been published that compared the hand sewing technique with the stapling technique for the esophagogastric anastomosis. ${ }^{124,128,139-146}$ In none of these clinical trials a statistically significant difference in the incidence of anastomotic leakage was reported. ${ }^{139-146}$ This finding was confirmed by two meta-analyses that did not find a difference in postoperative anastomotic leakage rates, other complications and postoperative mortality between hand-sewn or stapled anastomosis. ${ }^{147,148}$ Although, it has been suggested in a subgroup analysis of 302 patients that the stapled approach may reduce anastomotic leakage rates compared to a single-layer hand-sewn esophagogastric anastomosis (RR: 0.37, 95\% CI: 0.18-0.76, p<0.01). ${ }^{147}$

In two of the 10 clinical trials that compared the stapled and hand-sewn method, a 
statistically significant higher anastomotic stricture rate was found after stapled anastomotic construction. ${ }^{142,146}$ The other eight clinical trials did not report a difference in anastomotic strictures rates, ${ }^{139-141,143-145}$ although one study reported a non-significant difference towards more strictures in the stapled-group. ${ }^{143}$ A meta-analysis which included 7 of the 10 clinical trials found that a stapled anastomosis was associated with a higher anastomotic stricture rate (OR: 1.76, 95\% CI, 1.09-2.86; $\mathrm{p}=0.02) .{ }^{148}$ A possible explanation for this higher stricture rate is that the compression of tissue in the stapling device results in tissue ischemia between the staples, leading to fibrosis.

In summary, the current evidence suggests that hand-sewn and stapled anastomoses are equally safe with regard to leakage rates, other complications, and postoperative mortality. However, stapled anastomoses are likely associated with more stricture formation when compared to hand-sewn anastomoses.

\section{Lymphadenectomy for esophageal cancer}

Lymph node status is an important prognostic factor in esophageal carcinoma and an independent predictor of survival. ${ }^{149,150}$ Therefore, lymph node dissection is considered to be an integral part of oncological surgery. For esophagectomy, a transthoracic approach is generally considered to be optimal in oncological terms, as it allows for two-field lymphadenectomy with upper mediastinal dissection. ${ }^{94,151}$ The alternative, transhiatal esophagectomy, was designed to reduce post-operative morbidity and mortality by avoiding thoracotomy and is therefore often reserved for patients with considerable comorbidity and high risk of complications following a potential thoracotomy. ${ }^{152}$ A prospective randomized study comparing a transhiatal resection with an extended transthoracic approach for mid and distal esophageal adenocarcinomas showed that median overall, disease-free, and quality-adjusted survival did not differ statistically between the groups. ${ }^{93,153}$ However, there was a trend reported towards improved long-term survival at 5 years with the extended transthoracic approach (51\% for transthoracic approach versus $37 \%$ for the transhiatal approach in type I esophageal adenocarcinoma). ${ }^{93,153}$ Especially patients with 1 to 8 positive lymph nodes in the resection specimen showed a 5-year locoregional diseasefree survival advantage if operated via the transthoracic route compared to the transhiatal approach (23\% versus $64 \%, \mathrm{p}=0.02) .{ }^{153}$

A higher lymph node yield was associated with improved survival in an international cohort of 2303 esophageal cancer patients that underwent surgery alone. ${ }^{154}$ In contrast, two studies that performed post-hoc analyses on lymph node yield in patients treated with chemoradiotherapy followed by esophagectomy, suggested that lymph node yield was not associated with survival. ${ }^{155,156}$ It should be noted however, that these findings have to be 
interpreted with caution and the debate on the benefit of extensive lymphadenectomy has not yet been settled. It is well known that more lymph nodes are resected with a transthoracic approach compared to a transhiatal approach in patients that undergo surgery alone as well as neoadjuvant chemoradiation followed by surgery. ${ }^{157}$ In a recently published post-hoc analysis of a randomized controlled trial, a more favorable prognosis for the transthoracic approach was found in patients who underwent surgery alone, especially in patients with a limited number of positive lymph nodes. ${ }^{157}$ This benefit was absent in patients who underwent nCRT ${ }^{157}$, however this might be due to a lack of power for this post-hoc analysis. A recent population-based study from The Netherlands including 2698 esophageal cancer patients that underwent neoadjuvant chemoradiotherapy contradicted these results, and found a significant association between lymph node yield during esophagectomy and increased overall survival. ${ }^{158}$ In subgroup analyses of patients with squamous cell carcinoma and adenocarcinoma, $\mathrm{cN0}$ and $\mathrm{cN}+$, transthoracic and transhiatal approaches, and ypN0 and ypN+, a high lymph node yield remained significantly associated with increased overall survival. ${ }^{158}$

Moreover, a more extensive lymphadenectomy reflected by a higher lymph node yield during esophagectomy does not seem to decrease patients' short- and long-term health related quality of life, as reported by a Swedish population-based study including a total of 616 patients. ${ }^{159}$

Taken together, there is no evidence to date that justifies reduction in the quality or the extent of lymphadenectomy in esophageal cancer after neoadjuvant therapy. A transthoracic lymphadenectomy allows for optimal staging and locoregional disease control, and an inadequate lymphadenectomy may lead to positive lymph nodes being missed. ${ }^{160}$ Data regarding the lymph node status of individual lymph node stations would be of great importance before deciding to modify the extent of lymphadenectomy. The distribution of lymph node metastases is currently researched in a multinational observational cohort study with 58 participating centers (i.e, the TIGER study, NCT03222895).

\section{The role of surgery beyond locally advanced disease}

Since multimodality treatment for esophageal cancer is increasingly applied and can induce high degrees of tumor regression, even tumors that invade other adjacent structures such as aorta, vertebral body or trachea (T4b tumors) may be considered for resection in selected cases. ${ }^{161}$ Furthermore, it has been reported feasible and beneficial in terms of survival to perform a radical resection combined with off-pump descending aorta replacement and adjuvant chemotherapy in 47 patients with a T4b esophageal tumor invading the aorta. ${ }^{162}$ Also, there is evidence that patients with cT4b tumors can be safely treated with RAMIE 
after long-course chemoradiotherapy and restaging with PET-CT. ${ }^{161}$ A radical resection rate of $90 \%$ could be achieved in a case series of 10 patients. ${ }^{161}$

The selection of patients with oligometastases ( $<5$ metastases) for surgery is of particular interest. The therapeutic approach to these patients is currently undergoing a shift towards a more aggressive treatment including surgical resection. ${ }^{163} \mathrm{~A}$ radical surgical resection of the primary tumor and the metastases can be performed in patients who respond well to multimodal chemotherapy. The recently published results of the FLOT 3 study show that patients with limited metastatic disease after 4 cycles of neoadjuvant chemotherapy followed by routine surgical resection have a favorable survival compared to patients that received 8 cycles of chemotherapy and only a surgical intervention for a palliative indication. ${ }^{164}$ Patient selection for surgical resection was based on a realistic chance for R0 resection of the primary tumor, and the possibility of at least a macroscopic complete resection of the metastatic lesions as evaluated by endoscopy and CT or MRI scans. ${ }^{164}$ To identify patients that would benefit from such an aggressive treatment, response to systemic treatment and the 'test of time' (stable disease during follow-up) are currently the only useful markers. ${ }^{165}$ Ongoing randomized studies mainly focus on oligometastatic disease in patients with GEJ and gastric adenocarcinoma. The ongoing German FLOT 5 study (NCT02578368) compares chemotherapy followed by surgery versus chemotherapy alone in patients with GEJ or

gastric adenocarcinoma on overall survival. The German RENAISSANCE study ${ }^{166}$ (NCT02578368) includes patients with limited metastatic disease, defined as either retroperitoneal lymph node metastases only, or a maximum of one organ site that is potentially resectable or locally controllable with or without retroperitoneal lymph nodes. All patients will receive 4 cycles of FLOT (combined with trastuzumab if HER-2-positive), and are randomized to receive additional chemotherapy cycles or surgical resection of primary tumor and metastases followed by adjuvant chemotherapy. Overall survival will be used as primary endpoint. Lastly, the GASTRIPEC study (NCT02158988) evaluates the value of intraoperative hyperthermic intraperitoneal chemotherapy (HIPEC) in addition to perioperative chemotherapy and gastrectomy for patients with GEJ and gastric cancer with synchronous peritoneal carcinomatosis. ${ }^{167}$

\section{Enhanced Recovery After Esophagectomy in the postoperative setting}

After surgery, patients should first be able to mobilize as freely as possible to facilitate quick return to an acceptable activity level. To this end, the number of drains is preferably minimized and pain management should be effectively organized ${ }^{66}$ For gastrectomy for instance, routine placement of drains and nasogastric tubes is no longer recommended since their use has no proven benefit. ${ }^{60}$ However, esophagectomy usually involves an 
extensive thoracic procedure and temporary chest drainage remains to be considered necessary to prevent pulmonary compression by accumulation of air or fluid. ${ }^{66}$ Although direct evidence is lacking for esophagectomy, literature on open pulmonary surgery suggests that the use of a single drain is safe and that drains can be removed when producing less than 200 milliliters per day ${ }^{66,168,169}$ However, due to the sparsity of literature specifically addressing chest drainage after esophagectomy, postoperative drainage protocols vary widely and more research is needed to achieve consensus.

Besides limiting the number of drains that potentially hamper mobilization, adequate pain management is essential to facilitate fast recovery. ${ }^{170}$ Epidural analgesia is currently the gold standard after esophagectomy, as it was shown to be superior to systemic analgesia after open esophagectomy in terms of pain relief and pulmonary complications. ${ }^{171,172}$ Nonetheless, this technique can suffer from several potential problems such as failed placements, catheter occlusions or dislocations, and epidural related complications (e.g., hypotension, nausea, abscess, hematoma). ${ }^{173,174}$ With the rise of MIE, alternative techniques are increasingly investigated and applied ${ }^{96,175}$ In a recent systematic review and meta-analysis, paravertebral analgesia was suggested to be a serious option as it may provide equal pain control while possibly causing less complications such as hypotension, nausea and urinary retention when compared to epidural analgesia. ${ }^{175}$ However, well-designed prospective studies are warranted to provide more clarity on the optimal pain management modality.

Early resumption of oral intake is part of ERAS protocols to stimulate the return of bowel function. However, esophagectomy is somewhat unique due to the construction of a gastric conduit and a necessary vagotomy, which leads to delayed gastric emptying in $10-15 \%$ of patients. ${ }^{176}$ Previous studies suggested that it may be safe to allow clear liquids from postoperative day $1,{ }^{177}$ but feeding protocols vary widely. ${ }^{178,179}$ Currently, a randomized trial comparing early versus delayed start of oral intake after minimally invasive and hybrid esophagectomy is in its inclusion phase (i.e., the NUTRIENT II trial). ${ }^{180}$ The results of this study are expected to provide more clarity regarding the role of early oral intake in the context of enhanced recovery after esophagectomy.

\section{Conclusion and future directions}

With the implementation and favorable results of neoadjuvant therapy, curation is possible for an increased number of esophageal cancer patients. However, adequate staging and thorough examination of the general condition are essential to select patients who are eligible for, and would benefit from, esophagectomy. Over the last decades, minimally invasive techniques are increasingly used and have shown promising results. However, not only the surgical procedure itself, but the entire perioperative care pathway should be 
effectively organized in order to optimize surgical outcome. With this in mind, treatment of esophageal cancer with curative intent should be performed by a multidisciplinary team that is experienced in providing this type of care. 


\section{References}

1. Lagergren J, Smyth E, Cunningham D, Lagergren P. Oesophageal cancer. Lancet. 2017;390(10110):23832396.

2. Lordick F, Mariette C, Haustermans K, Obermannová R, Arnold D. Oesophageal cancer: ESMO Clinical Practice Guidelines for diagnosis, treatment and follow-up. Ann Oncol. 2016;27(suppl_5):v50-v57.

3. van Hagen P, Hulshof MCCM, van Lanschot JJB, et al. Preoperative Chemoradiotherapy for Esophageal or Junctional Cancer. N Engl J Med. 2012;366(22):2074-2084.

4. Cunningham D, Allum WWH, Stenning SSP, et al. Perioperative chemotherapy versus surgery alone for resectable gastroesophageal cancer. N Engl J Med. 2006;355(1):11-20.

5. Goense L, van Rossum PSN, Kandioler D, et al. Stage-directed individualized therapy in esophageal cancer. Ann N Y Acad Sci. 2016;1381(1):50-65.

6. Rice TW, Blackstone EH. Esophageal Cancer Staging. Thorac Surg Clin. 2013;23(4):461-469.

7. Findlay JM, Bradley KM, Maile EJ, et al. Pragmatic staging of oesophageal cancer using decision theory involving selective endoscopic ultrasonography, PET and laparoscopy. Br J Surg. 2015;102(12):1488-1499.

8. Zhang Y-Q, Chen T, Zhang C, et al. Endoscopic Submucosal Dissection for Superficial Proximal Esophageal Neoplasia is Highly Successful. Ann Surg. 2017;266(6):995-999.

9. Yang D, Zou F, Xiong S, Forde JJ, Wang Y, Draganov PV. Endoscopic submucosal dissection for early Barrett's neoplasia: a meta-analysis. Gastrointest Endosc. 2018;87(6):1383-1393.

10. Schölvinck D, Künzli H, Meijer S, et al. Management of patients with T1b esophageal adenocarcinoma: a retrospective cohort study on patient management and risk of metastatic disease. Surg Endosc. 2016;30(9):4102-4113.

11. Molena D, Schlottmann F, Boys JA, et al. Esophagectomy Following Endoscopic Resection of Submucosal Esophageal Cancer: a Highly Curative Procedure Even with Nodal Metastases. J Gastrointest Surg. 2017;21(1):62-67.

12. van der Horst S, van Hillegersberg R. Esophagectomy for Patients With Esophageal Cancer and Cervical Lymph Node Metastases (Node). https://clinicaltrials.gov/ct2/show/NCT02426879. Published 2015.

13. Herrmann E, Mertineit N, De Bari B, et al. Outcome of proximal esophageal cancer after definitive combined chemo-radiation: a Swiss multicenter retrospective study. Radiat Oncol. 2017;12(1):97.

14. Kassis ES, Kosinski AS, Ross P, Koppes KE, Donahue JM, Daniel VC. Predictors of anastomotic leak after esophagectomy: An analysis of the society of thoracic surgeons general thoracic database. Ann Thorac Surg. 2013;96(6):1919-1926.

15. Schmidt HM, Gisbertz SS, Moons J, et al. Defining Benchmarks for Transthoracic Esophagectomy: A Multicenter Analysis of Total Minimally Invasive Esophagectomy in Low Risk Patients. Ann Surg. 2017;266(5):814-821.

16. Goense L, van Rossum PSN, Weijs TJ, et al. Aortic Calcification Increases the Risk of Anastomotic Leakage After Ivor-Lewis Esophagectomy. Ann Thorac Surg. 2016;102(1):247-252.

17. van Rossum P, Haverkamp L, Verkooijen HM, van Leeuwen M, van Hillergersberg R, Ruurda J. Calcification of Arteries Supplying the Gastric Tube: A New Risk Factor for Anastomotic Leakage after Esophageal Surgery. Radiology. 2015;274(1):124-132.

18. Lainas P, Fuks D, Gaujoux S, et al. Preoperative imaging and prediction of oesophageal conduit necrosis after oesophagectomy for cancer. Br J Surg. 2017;104(10)1346-1354.

19. Zhao L, Zhao G, Li J, et al. Calcification of arteries supplying the gastric tube increases the risk of anastomotic leakage after esophagectomy with cervical anastomosis. J Thorac Dis. 2016;8(12):3551-3562.

20. Kechagias A, van Rossum PSN, Ruurda JP, van Hillegersberg R. Ischemic Conditioning of the Stomach in the Prevention of Esophagogastric Anastomotic Leakage After Esophagectomy. Ann Thorac Surg. 2016;101(4):1614-1623.

21. Schröder W, Hölscher AH, Bludau M, Vallböhmer D, Bollschweiler E, Gutschow C. Ivor-Lewis Esophagectomy With and Without Laparoscopic Conditioning of the Gastric Conduit. World J Surg. 2010;34(4):738-743.

22. Li S-J, Wang Z-Q, Li Y-J, et al. Diabetes mellitus and risk of anastomotic leakage after esophagectomy: a systematic review and meta-analysis. Dis Esophagus. 2017;30(6):1-12. 
23. Lagarde SM, Reitsma JB, Maris A-KD, et al. Preoperative Prediction of the Occurrence and Severity of Complications After Esophagectomy for Cancer With Use of a Nomogram. Ann Thorac Surg. 2008;85:19381946.

24. Wei R, Dong W, Shen H, et al. Predictive Effects of Lung function test on Postoperative Pneumonia in Squamous Esophageal Cancer. Sci Rep. 2016;6(1):23636.

25. Weijs TJ, Ruurda JP, Nieuwenhuijzen GA, Hillegersberg R van, Luyer MD. Strategies to reduce pulmonary complications after esophagectomy. World J Gastroenterol. 2013;19(39):6509.

26. Inoue J, Ono R, Makiura D, et al. Prevention of postoperative pulmonary complications through intensive preoperative respiratory rehabilitation in patients with esophageal cancer. Dis Esophagus. 2013;26(1):68-74.

27. van Adrichem EJ, Meulenbroek RL, Plukker JTM, Groen H, van Weert E. Comparison of two preoperative inspiratory muscle training programs to prevent pulmonary complications in patients undergoing esophagectomy: a randomized controlled pilot study. Ann Surg Oncol. 2014;21(7):2353-2360.

28. Valkenet K, van de Port IG, Dronkers JJ, de Vries WR, Lindeman E, Backx FJ. The effects of preoperative exercise therapy on postoperative outcome: a systematic review. Clin Rehabil. 2011;25(2):99-111.

29. Valkenet K, Trappenburg JC, Gosselink R, et al. Preoperative inspiratory muscle training to prevent postoperative pulmonary complications in patients undergoing esophageal resection (PREPARE study): study protocol for a randomized controlled trial. Trials. 2014;15(1):144.

30. Vlacich G, Samson PP, Perkins SM, et al. Treatment utilization and outcomes in elderly patients with locally advanced esophageal carcinoma: a review of the National Cancer Database. Cancer Med. 2017;6(12):28862896.

31. Nienhueser H, Kunzmann R, Sisic L, et al. Surgery of gastric cancer and esophageal cancer: Does age matter? J Surg Oncol. 2015;112(4):387-395.

32. Yamamoto M, Yamasaki M, Sugimoto K, et al. Risk Evaluation of Postoperative Delirium Using Comprehensive Geriatric Assessment in Elderly Patients with Esophageal Cancer. World J Surg. 2016;40(11):2705-2712.

33. Al-Batran S-E, Lorenzen S, Lilly E, Lorenzen S. Management of Locally Advanced Gastroesophageal Cancer Still a Multidisciplinary Global Challenge? Hematol Oncol Clin N Am. 2017;31:441-452.

34. Ychou M, Boige V, Pignon J, et al. Perioperative chemotherapy compared with surgery alone for resectable gastroesophageal adenocarcinoma: an FNCLCC and FFCD multicenter phase III trial. J Clin Oncol. 2011;29(13):1715.

35. Shapiro J, Lanschot JJB Van, Hulshof MCCM, Hagen P Van, Henegouwen MIVB, Wijnhoven BPL. Neoadjuvant chemoradiotherapy plus surgery versus surgery alone for oesophageal or junctional cancer (CROSS): long-term results of a randomised controlled trial. Lancet Oncol. 2015;16(9):1090-1098.

36. Al-Batran S, Homann N, Schmalenberg H, et al. Perioperative chemotherapy with docetaxel, oxaliplatin, and fluorouracil/leucovorin (FLOT) versus epirubicin, cisplatin, and fluorouracil or capecitabine (ECF/ ECX) for resectable gastric or gastroesophageal junction (GEJ) adenocarcinoma (FLOT4-AIO). J Clin Oncol. 2017;35(15_suppl):4004-4004.

37. Reynolds J, Preston S, O'Neill B, et al. ICORG 10-14: NEOadjuvant trial in Adenocarcinoma of the oEsophagus and oesophagoGastric junction International Study (Neo-AEGIS). BMC Cancer. 2017;17(1):401.

38. Hoeppner J, Lordick F, Brunner T, et al. ESOPEC: prospective randomized controlled multicenter phase III trial comparing perioperative chemotherapy (FLOT protocol) to neoadjuvant chemoradiation (CROSS protocol) in patients with adenocarcinoma of the esophagus (NCT02509286). BMC Cancer. 2016;16(1):503.

39. Tang H, Tan L, Shen Y, et al. CMISG1701: a multicenter prospective randomized phase III clinical trial comparing neoadjuvant chemoradiotherapy to neoadjuvant chemotherapy followed by minimally invasive esophagectomy in patients with locally advanced resectable esophageal squamous cell carcinoma (cT34aN0-1M0) (NCT03001596). BMC Cancer. 2017;17(1):450.

40. Mukherjee S, Hurt CN, Gwynne S, et al. NEOSCOPE: a randomised Phase II study of induction chemotherapy followed by either oxaliplatin/capecitabine or paclitaxel/carboplatin based chemoradiation as pre-operative regimen for resectable oesophageal adenocarcinoma. BMC Cancer. 2015;15(1):48.

41. Messager M, Mirabel X, Tresch E, et al. Preoperative chemoradiation with paclitaxel-carboplatin or with fluorouracil-oxaliplatin-folinic acid (FOLFOX) for resectable esophageal and junctional cancer: the PROTECT-1402, randomized phase 2 trial. BMC Cancer. 2016;16(1):318. 
42. Pasquali S, Guang Yim Ã, Vohra RS, et al. Survival After Neoadjuvant and Adjuvant Treatments Compared to Surgery Alone for Resectable Esophageal Carcinoma A Network Meta-analysis. Ann Surg. 2017;265(3):481-491.

43. Burt BM, Groth SS, Sada YH, et al. Utility of Adjuvant Chemotherapy After Neoadjuvant Chemoradiation and Esophagectomy for Esophageal Cancer. Ann Surg. 2017;266(2):297-304.

44. Noordman BJ, Wijnhoven BPL, Lagarde SM, et al. Active surveillance in clinically complete responders after neoadjuvant chemoradiotherapy for esophageal or junctional cancer. Dis Esophagus. 2017;30(12):1-8.

45. Oppedijk V, van der Gaast A, van Lanschot JJ, et al. Patterns of recurrence after surgery alone versus preoperative chemoradiotherapy and surgery in the CROSS trials. J Clin Oncol. 2014;32(5):385-391.

46. Alnaji RM, Du W, Gabriel E, et al. Pathologic Complete Response Is an Independent Predictor of Improved Survival Following Neoadjuvant Chemoradiation for Esophageal Adenocarcinoma. J Gastrointest Surg. 2016;20(9):1541-1546.

47. Donahue JM, Nichols FC, Li Z, et al. Complete Pathologic Response After Neoadjuvant Chemoradiotherapy for Esophageal Cancer Is Associated With Enhanced Survival. Ann Thorac Surg. 2009;87(2):392-399.

48. Berger AC, Farma J, Scott WJ, et al. Complete Response to Neoadjuvant Chemoradiotherapy in Esophageal Carcinoma Is Associated With Significantly Improved Survival. J Clin Oncol. 2005;23(19):4330-4337.

49. van Rossum PSN, Goense L, Meziani J, et al. Endoscopic biopsy and EUS for the detection of pathologic complete response after neoadjuvant chemoradiotherapy in esophageal cancer: a systematic review and meta-analysis. Gastrointest Endosc. 2016;83(5):866-879.

50. Kwee RM. Prediction of Tumor Response to Neoadjuvant Therapy in Patients with Esophageal Cancer with Use of ${ }^{18}$ F FDG PET: A Systematic Review. Radiology. 2010;254(3):707-717.

51. van Rossum PSN, Fried D V, Zhang L, et al. The Incremental Value of Subjective and Quantitative Assessment of 18F-FDG PET for the Prediction of Pathologic Complete Response to Preoperative Chemoradiotherapy in Esophageal Cancer. J Nucl Med. 2016;57(5):691-700.

52. Aoyagi T, Shuto K, Okazumi S, Shimada H, Kazama T, Matsubara H. Apparent diffusion coefficient values measured by diffusion-weighted imaging predict chemoradiotherapeutic effect for advanced esophageal cancer. Dig Surg. 2011;28(4):252-257.

53. De Cobelli F, Giganti F, Orsenigo E, et al. Apparent diffusion coefficient modifications in assessing gastrooesophageal cancer response to neoadjuvant treatment: Comparison with tumour regression grade at histology. Eur Radiol. 2013;23(8):2165-2174.

54. van Rossum PSN, van Lier ALHMW, van Vulpen M, et al. Diffusion-weighted magnetic resonance imaging for the prediction of pathologic response to neoadjuvant chemoradiotherapy in esophageal cancer. Radiother Oncol. 2015;115(2):163-170.

55. Wang L, Liu L, Han C, et al. The diffusion-weighted magnetic resonance imaging (DWI) predicts the early response of esophageal squamous cell carcinoma to concurrent chemoradiotherapy. Radiother Oncol. 2016;121:246-251.

56. Heethuis SE, van Rossum PSN, Lips IM, et al. Dynamic contrast-enhanced MRI for treatment response assessment in patients with oesophageal cancer receiving neoadjuvant chemoradiotherapy. Radiother Oncol. 2016;120(1):128-135.

57. Lordick F, Ott K, Krause B-J, et al. PET to assess early metabolic response and to guide treatment of adenocarcinoma of the oesophagogastric junction: the MUNICON phase II trial. Lancet Oncol. 2007;8(9):797-805.

58. Meyer zum Buschenfelde C, Herrmann K, Schuster T, et al. 18F-FDG PET-Guided Salvage Neoadjuvant Radiochemotherapy of Adenocarcinoma of the Esophagogastric Junction: The MUNICON II Trial. J Nucl Med. 2011;52(8):1189-1196.

59. Ljungqvist O, Young-Fadok T, Demartines N. The History of Enhanced Recovery After Surgery and the ERAS Society. J Laparoendosc Adv Surg Tech A. 2017;27(9):860-862.

60. Gustafsson UO, Scott MJ, Schwenk W, et al. Guidelines for perioperative care in elective colonic surgery: Enhanced Recovery After Surgery (ERAS(R)) Society recommendations. Clin Nutr. 2012;31(6):783-800.

61. Lassen K, Coolsen MM, Slim K, et al. Guidelines for perioperative care for pancreaticoduodenectomy: Enhanced Recovery After Surgery (ERAS(R)) Society recommendations. Clin Nutr. 2012;31(6):817-830.

62. Nygren J, Thacker J, Carli F, et al. Guidelines for perioperative care in elective rectal/pelvic surgery: Enhanced Recovery After Surgery (ERAS((R))) Society recommendations. World J Surg. 2013;37(2):285305. 
63. Nygren J, Thacker J, Carli F, et al. Guidelines for perioperative care in elective rectal/pelvic surgery: Enhanced Recovery After Surgery (ERAS(R)) Society recommendations. Clin Nutr. 2012;31(6):801-816.

64. Mortensen K, Nilsson M, Slim K, et al. Consensus guidelines for enhanced recovery after gastrectomy: Enhanced Recovery After Surgery (ERAS(R)) Society recommendations. Br J Surg. 2014;101(10):12091229.

65. Thorell A, MacCormick AD, Awad S, et al. Guidelines for Perioperative Care in Bariatric Surgery: Enhanced Recovery After Surgery (ERAS) Society Recommendations. World J Surg. 2016;40(9):2065-2083.

66. Findlay JM, Gillies RS, Millo J, Sgromo B, Marshall RE, Maynard ND. Enhanced recovery for esophagectomy: a systematic review and evidence-based guidelines. Ann Surg. 2014;259(3):413-431.

67. Markar SR, Schmidt H, Kunz S, Bodnar A, Hubka M, Low DE. Evolution of standardized clinical pathways: refining multidisciplinary care and process to improve outcomes of the surgical treatment of esophageal cancer. J Gastrointest Surg. 2014;18(7):1238-1246.

68. Aarts MA, Okrainec A, Glicksman A, Pearsall E, Victor JC, McLeod RS. Adoption of enhanced recovery after surgery (ERAS) strategies for colorectal surgery at academic teaching hospitals and impact on total length of hospital stay. Surg Endosc. 2012;26(2):442-450.

69. Younis J, Salerno G, Fanto D, Hadjipavlou M, Chellar D, Trickett JP. Focused preoperative patient stoma education, prior to ileostomy formation after anterior resection, contributes to a reduction in delayed discharge within the enhanced recovery programme. Int J Colorectal Dis. 2012;27(1):43-47.

70. Huddy JR, Huddy FMS, Markar SR, Tucker O. Nutritional optimization during neoadjuvant therapy prior to surgical resection of esophageal cancer-a narrative review. Dis Esophagus. October 2017.

71. Grotenhuis BA, Wijnhoven BP, Grune F, van Bommel J, Tilanus HW, van Lanschot JJ. Preoperative risk assessment and prevention of complications in patients with esophageal cancer. J Surg Oncol. 2010;101(3):270-278.

72. Zhong JX, Kang K, Shu XL. Effect of nutritional support on clinical outcomes in perioperative malnourished patients: a meta-analysis. Asia Pac J Clin Nutr. 2015;24(3):367-378.

73. Nozoe T, Kimura Y, Ishida M, Saeki H, Korenaga D, Sugimachi K. Correlation of pre-operative nutritional condition with post-operative complications in surgical treatment for oesophageal carcinoma. Eur J Surg Oncol. 2002;28(4):396-400.

74. Waitzberg DL, Saito H, Plank LD, et al. Postsurgical infections are reduced with specialized nutrition support. World J Surg. 2006;30(8):1592-1604.

75. Mariette C, De Botton ML, Piessen G. Surgery in esophageal and gastric cancer patients: what is the role for nutrition support in your daily practice? Ann Surg Oncol. 2012;19(7):2128-2134.

76. Mueller C, Compher C, Ellen DM. A.S.P.E.N. clinical guidelines: Nutrition screening, assessment, and intervention in adults. J Parenter Enter Nutr. 2011;35(1):16-24.

77. Arends J, Bachmann P, Baracos V, et al. ESPEN guidelines on nutrition in cancer patients. Clin Nutr. 2017;36(1):11-48.

78. Kingma BF, Steenhagen E, Ruurda JP, van Hillegersberg R. Nutritional aspects of enhanced recovery after esophagectomy with gastric conduit reconstruction. J Surg Oncol. 2017;116(5):623-629.

79. Jenkins TK, Lopez AN, Sarosi GA, Ben-David K, Thomas RM. Preoperative enteral access is not necessary prior to multimodality treatment of esophageal cancer. Surgery. 2018;163(4):770-776.

80. Yoshida N, Baba Y, Hiyoshi Y, et al. Duration of Smoking Cessation and Postoperative Morbidity After Esophagectomy for Esophageal Cancer: How Long Should Patients Stop Smoking Before Surgery? World J Surg. 2016;40(1):142-147.

81. Mantziari S, Hübner M, Demartines N, Schäfer M. Impact of preoperative risk factors on morbidity after esophagectomy: is there room for improvement? World J Surg. 2014;38(11):2882-2890.

82. Steenhagen E. Enhanced Recovery After Surgery: It's Time to Change Practice! Nutr Clin Pract. 2016;31(1):18-29.

83. Smith MD, McCall J, Plank L, Herbison GP, Soop M, Nygren J. Preoperative carbohydrate treatment for enhancing recovery after elective surgery. Cochrane database Syst Rev. 2014;(8):CD0091(8):CD009161.

84. Ljungqvist O. Modulating postoperative insulin resistance by preoperative carbohydrate loading. Best Pract Res Anaesthesiol. 2009;23(4):401-409.

85. Amer MA, Smith MD, Herbison GP, Plank LD, McCall JL. Network meta-analysis of the effect of preoperative carbohydrate loading on recovery after elective surgery. Br J Surg. 2017;104(3):187-197. 
86. Awad S, Varadhan KK, Ljungqvist O, Lobo DN. A meta-analysis of randomised controlled trials on preoperative oral carbohydrate treatment in elective surgery. Clin Nutr. 2013;32(1):34-44.

87. Ljungqvist O. ERAS--enhanced recovery after surgery: moving evidence-based perioperative care to practice. J Parenter Enter Nutr. 2014;38(5):559-566.

88. Pimenta GP, de Aguilar-Nascimento JE. Prolonged preoperative fasting in elective surgical patients: why should we reduce it? Nutr Clin Pract. 2014;29(1):22-28.

89. Maltby JR. Fasting from midnight--the history behind the dogma. Best Pract Res Anaesthesiol. 2006;20(3):363-378.

90. Mendelson CL. The aspiration of stomach contents into the lungs during obstetric anesthesia. Am J Obstet Gynecol. 1946;52:191-205.

91. Brady M, Kinn S, Stuart P. Preoperative fasting for adults to prevent perioperative complications. Cochrane database Syst Rev. 2003;(4)(4):CD004423.

92. Committee AS of A. Practice guidelines for preoperative fasting and the use of pharmacologic agents to reduce the risk of pulmonary aspiration: application to healthy patients undergoing elective procedures: an updated report by the American Society of Anesthesiologists Com. Anesthesiology. 2011;114(3):495-511.

93. Hulscher JBF, van Sandick JW, de Boer AGEM, et al. Extended Transthoracic Resection Compared with Limited Transhiatal Resection for Adenocarcinoma of the Esophagus. N Engl J Med. 2002;347(21):1662-1669.

94. McKeown KC. Total three-stage oesophagectomy for cancer of the oesophagus. Br J Surg. 1976;63(4):259262.

95. Lewis I. The surgical treatment of carcinoma of the oesophagus; with special reference to a new operation for growths of the middle third. Br J Surg. 1946;34:18-31.

96. Haverkamp L, Seesing MF, Ruurda JP, Boone J, V Hillegersberg R. Worldwide trends in surgical techniques in the treatment of esophageal and gastroesophageal junction cancer. Dis Esophagus. 2017;30(1):1-7.

97. Lennard TW, Shenton BK, Borzotta A, et al. The influence of surgical operations on components of the human immune system. Br J Surg. 1985;72(10):771-776.

98. Maas KW, Biere SS, van Hoogstraten IM, van der Peet DL, Cuesta MA. Immunological changes after minimally invasive or conventional esophageal resection for cancer: a randomized trial. World J Surg. 2014;38(1):131-137.

99. Veldkamp R, Kuhry E, Hop WC, et al. Laparoscopic surgery versus open surgery for colon cancer: shortterm outcomes of a randomised trial. Lancet Oncol. 2005;6(7):477-484.

100. Sauerland S, Jaschinski T, Neugebauer EA. Laparoscopic versus open surgery for suspected appendicitis. Cochrane database Syst Rev. 2010;(10):CD001(10):CD001546.

101. Bendixen M, Jorgensen OD, Kronborg C, Andersen C, Licht PB. Postoperative pain and quality of life after lobectomy via video-assisted thoracoscopic surgery or anterolateral thoracotomy for early stage lung cancer: a randomised controlled trial. Lancet Oncol. 2016;17(6):836-844.

102. Smithers BM, Gotley DC, Martin I, Thomas JM. Comparison of the outcomes between open and minimally invasive esophagectomy. Ann Surg. 2007;245(2):232-240.

103. Luketich JD, Alvelo-Rivera M, Buenaventura PO, et al. Minimally invasive esophagectomy: outcomes in 222 patients. Ann Surg. 2003;238(4):485-486.

104. Biere SS, van Berge Henegouwen MI, Maas KW, et al. Minimally invasive versus open oesophagectomy for patients with oesophageal cancer: a multicentre, open-label, randomised controlled trial. Lancet. 2012;379(9829):1887-1892.

105. Straatman J, van der Wielen N, Cuesta MA, et al. Minimally Invasive Versus Open Esophageal Resection: Three-year Follow-up of the Previously Reported Randomized Controlled Trial: the TIME Trial. Ann Surg. 2017;266(2):232-236.

106. Sihag S, Kosinski AS, Gaissert HA, Wright CD, Schipper PH. Minimally Invasive Versus Open Esophagectomy for Esophageal Cancer: A Comparison of Early Surgical Outcomes From The Society of Thoracic Surgeons National Database. Ann Thorac Surg. 2016;101(4):1281-1289.

107. Seesing MFJ, Gisbertz SS, Goense L, et al. A Propensity Score Matched Analysis of Open Versus Minimally Invasive Transthoracic Esophagectomy in the Netherlands. Ann Surg. August 2017.

108. Takeuchi H, Miyata H, Ozawa S, et al. Comparison of Short-Term Outcomes Between Open and Minimally Invasive Esophagectomy for Esophageal Cancer Using a Nationwide Database in Japan. Ann Surg Oncol. 2017;24(7):1821-1827.

109. Mamidanna R, Bottle A, Aylin P, Faiz O, Hanna GB. Short-term outcomes following open versus 
minimally invasive esophagectomy for cancer in England: a population-based national study. Ann Surg. 2012;255(2):197-203.

110. van Workum F, Stenstra MHBC, Berkelmans GHK, et al. Learning Curve and Associated Morbidity of Minimally Invasive Esophagectomy: A Retrospective Multicenter Study. Ann Surg. August 2017.

111. Briez N, Piessen G, Bonnetain F, et al. Open versus laparoscopically-assisted oesophagectomy for cancer: a multicentre randomised controlled phase III trial - the MIRO trial. BMC Cancer. 2011;11:310.

112. American Society of Clinical Oncology. ESMO 2017: MIRO Trial: 3-Year Outcomes Favor Laparoscopic Surgery for Esophageal Cancer. http://www.ascopost.com/News/58020. Published 2017. Accessed December 15, 2017.

113. Glatz T, Marjanovic G, Kulemann B, Sick O, Hopt UT, Hoeppner J. Hybrid minimally invasive esophagectomy vs. open esophagectomy: a matched case analysis in 120 patients. Langenbeck's Arch Surg. 2017;402(2):323-331.

114. Rinieri P, Ouattara M, Brioude G, et al. Long-term outcome of open versus hybrid minimally invasive Ivor Lewis oesophagectomy: a propensity score matched studydagger. Eur J cardio-thoracic Surg. 2017;51(2):223229.

115. Metcalfe C, Avery K, Berrisford R, et al. Comparing open and minimally invasive surgical procedures for oesophagectomy in the treatment of cancer: the ROMIO (Randomised Oesophagectomy: Minimally Invasive or Open) feasibility study and pilot trial. Health Technol Assess (Rockv). 2016;20(48):1-68.

116. van Hillegersberg R, Boone J, Draaisma WA, Broeders IA, Giezeman MJ, Borel Rinkes IH. First experience with robot-assisted thoracoscopic esophagolymphadenectomy for esophageal cancer. Surg Endosc. 2006;20(9):1435-1439.

117. Ruurda JP, van der Sluis PC, van der Horst S, van Hilllegersberg R. Robot-assisted minimally invasive esophagectomy for esophageal cancer: A systematic review. J Surg Oncol. 2015;112(3):257-265.

118. van der Sluis PC, Ruurda JP, Verhage RJ, et al. Oncologic Long-Term Results of Robot-Assisted Minimally Invasive Thoraco-Laparoscopic Esophagectomy with Two-Field Lymphadenectomy for Esophageal Cancer. Ann Surg Oncol. 2015;22 Suppl 3:S1350-6.

119. van der Horst S, Weijs TJ, Ruurda JP, et al. Robot-assisted minimally invasive thoraco-laparoscopic esophagectomy for esophageal cancer in the upper mediastinum. J Thorac Dis. 2017;9(Suppl 8):S834-S842.

120. van der Sluis PC, Ruurda JP, van der Horst S, et al. Robot-assisted minimally invasive thoraco-laparoscopic esophagectomy versus open transthoracic esophagectomy for resectable esophageal cancer, a randomized controlled trial (ROBOT trial). Trials. 2012;13:230.

121. Chasseray VM, Kiroff GK, Buard JL, Launois B. Cervical or thoracic anastomosis for esophagectomy for carcinoma. Surg Gynecol Obstet. 1989;169(1):55-62.

122. Walther B, Johansson J, Johnsson F, von Holstein CS, Zilling T. Cervical or Thoracic Anastomosis After Esophageal Resection and Gastric Tube Reconstruction. Ann Surg. 2003;238(6):803-814.

123. Okuyama M, Motoyama S, Suzuki H, Saito R, Maruyama K, Ogawa J-I. Hand-sewn cervical anastomosis versus stapled intrathoracic anastomosis after esophagectomy for middle or lower thoracic esophageal cancer: a prospective randomized controlled study. Surg Today. 2007;37(11):947-952. $124 . \quad$ R i b e t M, Debrueres B, Lecomte-Houcke M. Resection for advanced cancer of the thoracic esophagus: cervical or thoracic anastomosis? Late results of a prospective randomized study. J Thorac Cardiovasc Surg. 1992;103(4):784-789.

125. Markar SR, Arya S, Karthikesalingam A, Hanna GB. Technical Factors that Affect Anastomotic Integrity Following Esophagectomy: Systematic Review and Meta-analysis. Ann Surg Oncol. 2013;20(13):4274-4281.

126. Biere SSAYS, Maas KWW, Cuesta MAA, van der Peet DLL. Cervical or thoracic anastomosis after esophagectomy for cancer: a systematic review and meta-analysis. Dig Surg. 2011;28(1):29-35.

127. Walther B, Johansson J, Johnsson F, Von Holstein CS, Zilling T. Cervical or thoracic anastomosis after esophageal resection and gastric tube reconstruction: a prospective randomized trial comparing sutured neck anastomosis with stapled intrathoracic anastomosis. Ann Surg. 2003;238(6):803-804.

128. Okuyama M, Motoyama S, Suzuki H, Saito R, Maruyama K, Ogawa J. Hand-sewn cervical anastomosis versus stapled intrathoracic anastomosis after esophagectomy for middle or lower thoracic esophageal cancer: a prospective randomized controlled study. Surg Today. 2007;37(11):947-952.

129. Gooszen JAH, Goense L, Gisbertz SS, Ruurda JP, van Hillegersberg R, van Berge Henegouwen MI. Intrathoracic versus cervical anastomosis and predictors of anastomotic leakage after oesophagectomy for cancer. Br J Surg. 2018;105(5):552-560. 
130. Gronnier C, Trechot B, Duhamel A, et al. Impact of neoadjuvant chemoradiotherapy on postoperative outcomes after esophageal cancer resection: results of a European multicenter study. Ann Surg. 2014;260(5):761-764.

131. Klink CD, Binnebosel M, Otto J, et al. Intrathoracic versus cervical anastomosis after resection of esophageal cancer: a matched pair analysis of 72 patients in a single center study. World J Surg Oncol. 2012;10:159.

132. Gockel I, Heckhoff S, Messow CM, Kneist W, Junginger T. Transhiatal and transthoracic resection in adenocarcinoma of the esophagus: does the operative approach have an influence on the long-term prognosis? World J Surg Oncol. 2005;3:40.

133. Boyle MJ, Franceschi D, Livingstone AS. Transhiatal versus transthoracic esophagectomy: complication and survival rates. Am Surg. 1999;65(12):1132-1137.

134. van Heijl M, van Wijngaarden AK, Lagarde SM, Busch OR, van Lanschot JJ, van Berge Henegouwen MI. Intrathoracic manifestations of cervical anastomotic leaks after transhiatal and transthoracic oesophagectomy. Br J Surg. 2010;97(5):726-731.

135. van Rossum PSN, Haverkamp L, Carvello M, Ruurda JP, van Hillegersberg R. Management and outcome of cervical versus intrathoracic manifestation of cervical anastomotic leakage after transthoracic esophagectomy for cancer. Dis Esophagus. 2017;30(1):1-8.

136. Degisors S, Pasquer A, Renaud F, et al. Are Thoracotomy and/or Intrathoracic Anastomosis Still Predictors of Postoperative Mortality After Esophageal Cancer Surgery?: A Nationwide Study. Ann Surg. 2017;266(5):854-862.

137. Gronnier C, Trechot B, Duhamel A, et al. Impact of neoadjuvant chemoradiotherapy on postoperative outcomes after esophageal cancer resection: results of a European multicenter study. Ann Surg. 2014;260(5):761-764.

138. van Workum F, Bouwense SAW, Luyer MDP, et al. Intrathoracic versus Cervical ANastomosis after minimally invasive esophagectomy for esophageal cancer: study protocol of the ICAN randomized controlled trial. Trials. 2016;17(1):505.

139. Craig SR, Walker WS, Cameron EW, Wightman AJ. A prospective randomized study comparing stapled with handsewn oesophagogastric anastomoses. J R Coll Surg Edinb. 1996;41(1):17-19.

140. Hsu HH, Chen JS, Huang PM, Lee JM, Lee YC. Comparison of manual and mechanical cervical esophagogastric anastomosis after esophageal resection for squamous cell carcinoma: a prospective randomized controlled trial. Eur J Cardiothorac Surg. 2004;25(6):1097-1101.

141. Laterza E, de’Manzoni G, Veraldi GF, Guglielmi A, Tedesco P, Cordiano C. Manual compared with mechanical cervical oesophagogastric anastomosis: a randomised trial. Eur J Surg. 1999;165(11):10511054.

142. Law S, Fok M, Chu KM, Wong J. Comparison of hand-sewn and stapled esophagogastric anastomosis after esophageal resection for cancer: a prospective randomized controlled trial. Ann Surg. 1997;226(2):169-173.

143. Luechakiettisak P, Kasetsunthorn S. Comparison of hand-sewn and stapled in esophagogastric anastomosis after esophageal cancer resection: a prospective randomized study. J Med Assoc Thai. 2008;91(5):681-685.

144. Saluja SS, Ray S, Pal S, et al. Randomized trial comparing side-to-side stapled and hand-sewn esophagogastric anastomosis in neck. J Gastrointest Surg. 2012;16(7):1287-1295.

145. Valverde A, Hay JM, Fingerhut A, Elhadad A. Manual versus mechanical esophagogastric anastomosis after resection for carcinoma: a controlled trial. French Associations for Surgical Research. Surgery. 1996;120(3):476-483.

146. Zhang YS, Gao BR, Wang HJ, et al. Comparison of anastomotic leakage and stricture formation following layered and stapler oesophagogastric anastomosis for cancer: a prospective randomized controlled trial. J Int Med Res. 2010;38(1):227-233.

147. Liu QX, Min JX, Deng XF, Dai JG. Is hand sewing comparable with stapling for anastomotic leakage after esophagectomy? A meta-analysis. World J Gastroenterol. 2014;20(45):17218-17226.

148. Markar SR, Karthikesalingam A, Vyas S, Hashemi M, Winslet M. Hand-Sewn Versus Stapled Oesophagogastric Anastomosis: Systematic Review and Meta-analysis. J Gastrointest Surg. 2011;15(5):876-884

149. Rice TW, Chen L-Q, Hofstetter WL, et al. Worldwide Esophageal Cancer Collaboration: pathologic staging data. Dis Esophagus. 2016;29(7):724-733.

150. Mariette C, Piessen G, Briez N, Triboulet JP. The Number of Metastatic Lymph Nodes and the Ratio Between Metastatic and Examined Lymph Nodes Are Independent Prognostic Factors in Esophageal Cancer Regardless of Neoadjuvant Chemoradiation or Lymphadenectomy Extent. Ann Surg. 2008;247(2):365-371. 
151. Visbal AL, Allen MS, Miller DL, Deschamps C, Trastek VF, Pairolero PC. Ivor Lewis esophagogastrectomy for esophageal cancer. Ann Thorac Surg. 2001;71(6):1803-1808.

152. Orringer MB. Transhiatal esophagectomy without thoracotomy for carcinoma of the thoracic esophagus. Ann Surg. 1984;200(3):282-288.

153. Omloo JMT, Lagarde SM, Hulscher JBF, et al. Extended Transthoracic Resection Compared With Limited Transhiatal Resection for Adenocarcinoma of the Mid/Distal Esophagus. Ann Surg. 2007;246(6):992-1001.

154. Peyre CG, Hagen JA, DeMeester SR, et al. The number of lymph nodes removed predicts survival in esophageal cancer: an international study on the impact of extent of surgical resection. Ann Surg. 2008;248(4):549-556.

155. Robb W, Dahan L, Mornex F, et al. Impact of neoadjuvant chemoradiation on lymph node status in esophageal cancer: post hoc analysis of a randomized controlled trial. Ann Surg. 2015;261(5):902-908.

156. Talsma K, Wijnhoven B, van Lanschot J, van Berge Henegouwen M. Impact of Neoadjuvant Chemoradiation on Lymph Node Status in Esophageal Cancer. Ann Surg. 2015;266(6):e52-e53.

157. Noordman BJ, Van Klaveren D, van Berge Henegouwen MI, et al. Impact of Surgical Approach on Longterm Survival in Esophageal Adenocarcinoma Patients With or Without Neoadjuvant Chemoradiotherapy. Ann Surg. 2017;267(5):892-897.

158. Visser E, van Rossum PSN, Ruurda JP, van Hillegersberg R. Impact of Lymph Node Yield on Overall Survival in Patients Treated With Neoadjuvant Chemoradiotherapy Followed by Esophagectomy for Cancer. Ann Surg. 2017;266(5):1.

159. Schandl A, Johar A, Lagergren J, Lagergren P. Lymphadenectomy and health-related quality of life after oesophageal cancer surgery: a nationwide, population-based cohort study. BMJ Open. 2016;6(8):e012624.

160. Robb WB, Maillard E, Mariette C. Lymph Node Status After Neoadjuvant Chemoradiotherapy for Esophageal Cancer. Ann Surg. 2017;266(6):e53-e54..

161. van Hillegersberg R, Seesing MFJ, Brenkman HJF, Ruurda JP. Robot-assisted minimally invasive esophagectomy. Der Chir. 2017;88(S1):7-11. 162. Cong Z, Diao Q, Yi J, et al. Esophagectomy Combined With Aortic Segment Replacement for Esophageal Cancer Invading the Aorta. Ann Thorac Surg. 2014;97(2):460-466.

163. Schmidt T, Mönig SP. Therapeutisches Vorgehen beim oligometastasierten Magen- und Ösophaguskarzinom. Der Chir. November 2017:1-9.

164. Al-Batran S-E, Homann N, Pauligk C, et al. Effect of Neoadjuvant Chemotherapy Followed by Surgical Resection on Survival in Patients With Limited Metastatic Gastric or Gastroesophageal Junction Cancer. JAMA Oncol. 2017;3(9):1237.

165. Chiapponi C, Berlth F, Plum PS, et al. Oligometastatic Disease in Upper Gastrointestinal Cancer - How to Proceed? Visc Med. 2017;33:31-34.

166. Mueller D, Moenig S, Vogel A, et al. The "RENAISSANCE" Trial: Effect of chemotherapy alone vs. chemotherapy followed by surgical resection on survival and quality of life in patients with limitedmetastatic adenocarcinoma of the stomach or esophagogastric junction. J Clin Oncol. 2017;35(15_ suppl):TPS4140.

167. Rau B, Loeffler M, Rau H, et al. Perioperative chemotherapy and cytoreductive surgery with versus without HIPEC in gastric cancer with limited peritoneal metastases: A randomized phase III study (GASTRIPEC). J Clin Oncol. 2015;33(15_suppl):TPS4132.

168. Johansson J, Lindberg CG, Johnsson F, von Holstein CS, Zilling T, Walther B. Active or passive chest drainage after oesophagectomy in 101 patients: a prospective randomized study. Br J Surg. 1998;85(8):11431146.

169. Gomez-Caro A, Roca MJ, Torres J, et al. Successful use of a single chest drain postlobectomy instead of two classical drains: a randomized study. Eur J cardio-thoracic Surg. 2006;29(4):562-566.

170. Richardson J, Sabanathan S, Mearns AJ, Evans CS, Bembridge J, Fairbrass M. Efficacy of pre-emptive analgesia and continuous extrapleural intercostal nerve block on post-thoracotomy pain and pulmonary mechanics. J Cardiovasc Surg (Torino). 1994;35(3):219-228.

171. Davies RG, Myles PS, Graham JM. A comparison of the analgesic efficacy and side-effects of paravertebral vs epidural blockade for thoracotomy--a systematic review and meta-analysis of randomized trials. $\mathrm{Br} \mathrm{J}$ Anaesth. 2006;96(4):418-426.

172. Cense HA, Lagarde SM, de Jong K, et al. Association of no epidural analgesia with postoperative morbidity and mortality after transthoracic esophageal cancer resection. J Am Coll Surg. 2006;202(3):395-400. 
173. Hermanides J, Hollmann MW, Stevens MF, Lirk P. Failed epidural: causes and management. Br J Anaesth. 2012;109(2):144-154.

174. Miller RD. Chapter 43: Spinal, Epidural, and Caudal Anesthesia. In: Brown DL, ed. 6th ed. Philadelphia, Pennsylvania, USA: Elsevier Churchill Livingstone; 2005:1653.

175. Visser E, Marsman M, van Rossum PSN, et al. Postoperative pain management after esophagectomy: a systematic review and meta-analysis. Dis Esophagus. 2017;30(10):1-11.

176. Poghosyan T, Gaujoux S, Chirica M, Munoz-Bongrand N, Sarfati E, Cattan P. Functional disorders and quality of life after esophagectomy and gastric tube reconstruction for cancer. J Visc Surg. 2011;148(5):e32735.

177. Weijs TJ, Berkelmans GH, Nieuwenhuijzen GA, et al. Immediate Postoperative Oral Nutrition Following Esophagectomy: A Multicenter Clinical Trial. Ann Thorac Surg. 2016;102(4):1141-1148.

178. Eberhard KE, Achiam MP, Rolff HC, Belmouhand M, Svendsen LB, Thorsteinsson M. Comparison of "Nil by Mouth" Versus Early Oral Intake in Three Different Diet Regimens Following Esophagectomy. World J Surg. January 2017.

179. Giacopuzzi S, Weindelmayer J, Treppiedi E, et al. Enhanced recovery after surgery protocol in patients undergoing esophagectomy for cancer: a single center experience. Dis Esophagus. 2017;30(4):1-6.

180. Berkelmans GH, Wilts BJ, Kouwenhoven EA, et al. Nutritional route in oesophageal resection trial II (NUTRIENT II): study protocol for a multicentre open-label randomised controlled trial. BMJ Open. 2016;6(8):e011979-2016-011979. 


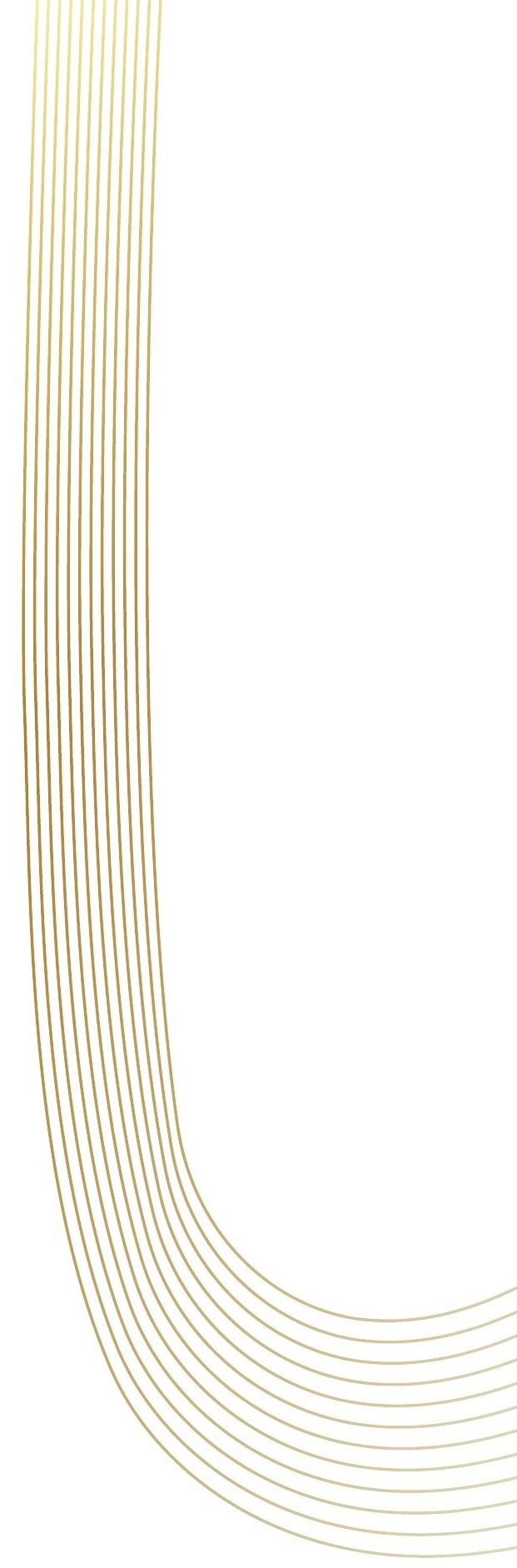


Frequency of surgical resection after starting neoadjuvant chemoradiotherapy in patients with esophageal cancer: a population-based cohort study

European Journal of Surgical Oncology 2019;45(10):1919-1925.

A.S. Borggreve, P.S.N. van Rossum, S. Mook, N. Haj Mohammad, R. van Hillegersberg, J.P. Ruurda 


\section{Abstract}

\section{Background}

Neoadjuvant chemoradiotherapy (nCRT) for resectable esophageal cancer is accompanied by the risk of treatment-related toxicity. The aim of this population-based cohort study was to provide insight in patients who do not proceed to surgical resection after starting nCRT.

\section{Methods}

Patients who started nCRT for primary esophageal cancer diagnosed in 2015 and 2016 were selected from the nationwide population-based cancer registry. Outcome measurements included omission from surgical resection, reasons for omission of surgical resection, mortality during nCRT ( $\leq 90$ days after ending $\mathrm{nCRT}$ ) and 1-year overall survival. Multivariable logistic regression analyses were performed to identify predictive factors for omission of surgical resection.

\section{Results}

A total of 1521 patients were included, of whom 215 (14.1\%) did not undergo surgical resection after starting nCRT. Age (OR: 1.04, 95\% CI: 1.01-1.06), BMI (OR: 0.95, 95\% CI: 0.90-0.99), WHO performance status (WHO 1: OR: 1.62, 95\% CI: 1.16-2.62 and WHO 2: OR: 3.53, 95\% CI: 1.68-7.41) and clinical N status (cN2: OR: 1.57, 95\% CI: 1.04-2.37 and cN3: OR: 2.52 , 95\% CI: 1.14-5.55) were significantly associated with omission from surgery. The most frequently reported reasons for omission from surgery were disease progression (44.3\%) and physical functioning (22.8\%). During nCRT or within the subsequent waiting period to surgery, 38 patients (2.5\%) deceased. One year overall survival of the patients who underwent nCRT followed by surgical resection was $94.9 \%$, and $73.5 \%$ in the patients who did not undergo surgical resection following nCRT.

\section{Conclusions}

One in 7 patients who started nCRT for esophageal cancer do not proceed to surgical resection and have a decreased one year overall survival compared to patients who do proceed to surgical resection. Mortality during nCRT is considerable. 


\section{Introduction}

Esophagectomy with lymphadenectomy remains the cornerstone of curative treatment for locally advanced esophageal cancer. Improved quality measures ${ }^{1}$, such as centralization ${ }^{2-5}$ and enhanced perioperative care, ${ }^{6,7}$ have resulted in decreased morbidity and mortality rates following surgical resection. Neoadjuvant chemoradiotherapy (nCRT) has been increasingly recognized as an integral component of the curative treatment of esophageal cancer, improving overall survival compared to surgery alone. ${ }^{8-11}$ Despite its prognostic benefits, nCRT is nevertheless not without the risk of treatment-associated toxicities.

Several studies have focused on complications during neoadjuvant therapy and their effects on surgical morbidity and short- and long-term survival. ${ }^{12-14}$ This is especially relevant when these toxic effects prevent patients from undergoing surgical resection. Well-known randomized controlled intervention trials report omission from surgical resection after starting nCRT in up to $15 \%$ of patients $\left(1.8 \%{ }^{8}\right.$ to $\left.15.4 \%{ }^{11}\right)$ and mortality rates of $<1 \%$ during nCRT $\left(0 \%^{11}\right.$ to $\left.0.6 \%{ }^{8}\right)$. As trial populations are carefully selected based on strict inclusion criteria - e.g. patients with limited comorbidities -, the representativeness of these results for the general cancer population might be compromised ${ }^{15}$.

Therefore, the aim of this population-based cohort study was to describe the patients with esophageal cancer who do not proceed to surgical resection after starting nCRT, as well as to identify potential predictive factors for unplanned omission from surgical resection and to describe the reasons for unplanned omission from surgical resection. Better understanding of the factors placing patients at higher risk of not proceeding to surgical resection may ultimately improve clinical decision making. Moreover, the mortality rate during $\mathrm{nCRT}$, or in the subsequent waiting period to surgery for esophageal cancer was described.

\section{Material and methods}

\section{Study design}

This population-based observational cohort study included data from the Netherlands Cancer Registry (NCR). The NCR registers cases based on notification of all newly diagnosed malignancies in the Netherlands by the national automated pathological archive (PALGA). Additional sources are the national registry of hospital discharge diagnoses and radiotherapy institutions. The NCR stores data on patient, tumor and treatment characteristics. Data is routinely extracted from hospital records by trained data managers. Information on a patient's vital status is updated through an annual linkage with the municipal personal records database, in which all deceased and emigrated persons in the Netherlands are registered. 
This study was approved by the Privacy Review Board of the NCR. According to the Central Committee on Research involving Human Subjects, this type of study does not require approval from an ethics committee in the Netherlands.

\section{Study population}

For the purpose of this study, all patients with esophageal cancer in the Netherlands who started nCRT in 2015 and 2016 were selected. Patients with distant metastases before the start of nCRT (M1) and a histologic tumor type other than adenocarcinoma or squamous cell carcinoma were excluded. Data on patient and treatment-related characteristics, histopathological characteristics, and follow-up were extracted from the NCR. As exact chemotherapy and radiotherapy regimens, as well as initial treatment plans for individual patients have been registered by the NCR from 2015 onwards, patients could specifically be selected that started nCRT according to the CROSS regimen (carboplatin targeted at an area under the curve (AUC) of $2 \mathrm{mg} / \mathrm{ml} / \mathrm{min}$ and paclitaxel at a dose of $50 \mathrm{mg} / \mathrm{m}^{2}$, weekly during 5 weeks, and concurrent radiotherapy with a total radiation dose of $41.4 \mathrm{~Gy}$ in 23 fractions of $\left.1.8 \mathrm{~Gy}^{8}\right)$.

Patients were diagnosed according to the Dutch national guidelines for patients with esophageal cancer. ${ }^{16}$ The diagnostic work-up consisted of at least endoscopy with tumor biopsy and computed tomography (CT) with or without positron emission tomography (PET). Patients were staged according to the $7^{\text {th }}$ edition of the Union for International Cancer Control (UICC) TNM staging system. ${ }^{17}$

Surgery was defined as actual surgical resection, by means of a transhiatal or transthoracic esophagectomy, either open or minimally invasive, but always combined with a lymphadenectomy. The choice of technique was at the discretion of the treating surgeon. CROSS eligibility criteria were defined as age 18 - 75 years, a WHO performance status of $\leq 2$, clinical T status $\leq \mathrm{T} 3$, tumor length $\leq 8 \mathrm{~cm}$ and no history of other cancer. All patients in this cohort were M0. No data was available in the NCR to account for the remaining eligibility criteria of the CROSS trial (i.e. lost $10 \%$ or less of body weight, no previous radiotherapy or chemotherapy, adequate hematologic, renal, hepatic, and pulmonary function). ${ }^{8}$

\section{Outcome measures}

Whether or not surgical resection was performed was routinely registered by the NCR. Reasons for omission from surgical resection in patients who started nCRT were registered based on standardized categories for patients diagnosed in 2015 only.

Mortality during nCRT was defined as all-cause mortality during nCRT, or within the subsequent waiting time to surgery ( $\leq 90$ days after the end date of nCRT), and always before the intended surgical resection. 
Follow-up time was measured from start of nCRT, and was completed for all patients until January $1^{\text {st }}, 2018$. Survival time was defined as time from diagnosis to death or until January $1^{\text {st }}, 2018$ for patients who were still alive.

\section{Statistical analyses}

Patient and treatment-related characteristics were described as counts with percentages or mean ( \pm standard deviation $[S D]$ ). The number of patients who did not undergo surgical resection after starting nCRT and the corresponding reasons for not undergoing surgical resection (only of the patients diagnosed in 2015), as well as the mortality during nCRT or the subsequent waiting period was described.

To be able to identify potential predictive factors for omission from surgical resection after starting nCRT, missing values were imputed using multiple imputation (Multivariate Imputation by Chained Equations, 30 imputed datasets with a maximum number of 20 iterations for each imputation). ${ }^{18,19}$ Variables to be entered in the multivariable logistic regression model with omission from surgery as outcome were based on clinical reasoning and literature review, and consisted of age, sex, body mass index (BMI), performance status, number of comorbidity categories, clinical T status, clinical N status, tumor histology and whether or not patients met the eligibility criteria of the CROSS trial. No further predictor selection was performed. Model coefficients were pooled using Rubin's rules.

Survival curves were obtained using the Kaplan-Meier method.

Statistical analyses were performed using R open-source software ('mice' and 'glm' packages, version 3.5.1) and GraphPad Prism version 7.04 (La Jolla, California, USA). For the multivariable logistic regression model, the p-value was deemed to be less than or equal to 0.05 , and the effect was said to be significant, if the confidence interval failed to include 1 .

\section{Results}

\section{Study population}

The study cohort included 1521 patients who started nCRT for potentially resectable esophageal cancer in the Netherlands in 2015 and 2016. A complete overview of patient and treatment-related characteristics is presented in Table 1 . The majority of patients was male $(78.3 \%, 1191 / 1521)$ with a mean age of 65.1 years $(\mathrm{SD} \pm 8.8)$ and a WHO performance status at diagnosis of $0(52.5 \%, 798 / 1521)$ or $1(30.4 \%, 462 / 1521)$. Most tumors were distal esophageal adenocarcinomas with clinical T2-3 status and accompanied by at least 1 lymph node metastasis (clinical N1-3).

A total of 457 patients (30.0\%) exceeded the original eligibility criteria of the CROSS trial based on one or a combination of the following characteristics: age $\geq 75$ years $(n=211)$, cT4 
Table 1. Baseline characteristics of patients who started neoadjuvant chemoradiotherapy (nCRT) for esophageal cancer in the Netherlands in 2015 and 2016

\begin{tabular}{|c|c|c|c|c|c|c|}
\hline \multirow[t]{2}{*}{ Characteristics } & \multicolumn{2}{|c|}{ All } & \multicolumn{2}{|c|}{$\begin{array}{c}\text { Omission from } \\
\text { surgical resection }\end{array}$} & \multicolumn{2}{|c|}{$\begin{array}{c}\text { Deceased during } \\
\text { nCRT }^{*}\end{array}$} \\
\hline & $\mathrm{n}=1521$ & $(\%)$ & $\mathrm{n}=215$ & $(\%)$ & $\mathrm{n}=38$ & $(\%)$ \\
\hline Age, years $($ mean $\pm S D)$ & \multicolumn{2}{|c|}{$65.1 \pm 8.8$} & \multicolumn{2}{|c|}{$67.6 \pm 9.4$} & \multicolumn{2}{|c|}{$68.0 \pm 8.3$} \\
\hline \multicolumn{7}{|l|}{ Sex } \\
\hline Male & 1191 & $78.3 \%$ & 169 & $78.6 \%$ & 34 & $89.5 \%$ \\
\hline Female & 330 & $21.7 \%$ & 46 & $21.4 \%$ & 4 & $10.5 \%$ \\
\hline $\mathbf{B M I}, \mathbf{k g} / \mathbf{m}^{2}($ mean $\pm \mathrm{SD})$ & \multicolumn{2}{|c|}{$26.1 \pm 4.3$} & \multicolumn{2}{|c|}{$25.1 \pm 4.2$} & \multicolumn{2}{|c|}{$25.9 \pm 4.7$} \\
\hline Unknown & 483 & $31.8 \%$ & 126 & $58.6 \%$ & 15 & $39.5 \%$ \\
\hline \multicolumn{7}{|l|}{ WHO performance status } \\
\hline 0 & 798 & $52.5 \%$ & 86 & $40.0 \%$ & 12 & $31.6 \%$ \\
\hline 1 & 462 & $30.4 \%$ & 81 & $37.7 \%$ & 15 & $39.5 \%$ \\
\hline 2 & 38 & $2.5 \%$ & 13 & $6.0 \%$ & 5 & $13.2 \%$ \\
\hline 3 & 1 & $0.1 \%$ & 1 & $0.5 \%$ & 0 & $0.0 \%$ \\
\hline Unknown & 222 & $14.6 \%$ & 34 & $15.8 \%$ & 6 & $15.8 \%$ \\
\hline \multicolumn{7}{|l|}{ Comorbidity categories $^{\dagger}$} \\
\hline None & 419 & $27.5 \%$ & 45 & $20.9 \%$ & 4 & $10.5 \%$ \\
\hline One category & 448 & $29.5 \%$ & 54 & $25.1 \%$ & 7 & $18.4 \%$ \\
\hline Two or more categories & 516 & $33.9 \%$ & 94 & $43.7 \%$ & 22 & $57.9 \%$ \\
\hline Unknown & 138 & $9.1 \%$ & 22 & $10.2 \%$ & 5 & $13.2 \%$ \\
\hline \multicolumn{7}{|l|}{ Specific comorbidities } \\
\hline Hypertension & 497 & $32.7 \%$ & 78 & $36.3 \%$ & 17 & $44.7 \%$ \\
\hline $\begin{array}{l}\text { Cardiovascular comorbidities } \\
\text { (other than hypertension) }\end{array}$ & 398 & $26.2 \%$ & 76 & $35.3 \%$ & 19 & $50.0 \%$ \\
\hline Diabetes mellitus & 204 & $13.4 \%$ & 40 & $18.6 \%$ & 8 & $21.1 \%$ \\
\hline Pulmonary comorbidities & 199 & $13.1 \%$ & 35 & $16.3 \%$ & 7 & $18.4 \%$ \\
\hline History of cancer & 169 & $11.1 \%$ & 24 & $11.2 \%$ & 5 & $13.2 \%$ \\
\hline Gastrointestinal comorbidities & 84 & $5.5 \%$ & 14 & $6.5 \%$ & 4 & $10.5 \%$ \\
\hline Renal & 22 & $1.4 \%$ & 9 & $4.2 \%$ & 1 & $2.6 \%$ \\
\hline Liver & 17 & $1.1 \%$ & 1 & $0.5 \%$ & 0 & $0.0 \%$ \\
\hline \multicolumn{7}{|l|}{ Tumor location } \\
\hline Proximal esophagus & 14 & $0.9 \%$ & 6 & $2.8 \%$ & 2 & $5.6 \%$ \\
\hline Middle esophagus & 176 & $11.6 \%$ & 29 & $13.5 \%$ & 3 & $7.9 \%$ \\
\hline Distal esophagus & 1162 & $76.4 \%$ & 146 & $67.9 \%$ & 25 & $65.8 \%$ \\
\hline Esophagus not otherwise specified & 52 & $3.4 \%$ & 13 & $6.0 \%$ & 3 & $7.9 \%$ \\
\hline Cardia & 117 & $7.7 \%$ & 21 & $9.8 \%$ & 5 & $13.2 \%$ \\
\hline \multicolumn{7}{|l|}{ Histology } \\
\hline Adenocarcinoma & 1202 & $79.0 \%$ & 159 & $74.0 \%$ & 30 & $78.9 \%$ \\
\hline Squamous cell carcinoma & 319 & $21.0 \%$ & 56 & $26.0 \%$ & 8 & $21.1 \%$ \\
\hline \multicolumn{7}{|l|}{ Tumor differentiation } \\
\hline Well & 51 & $3.4 \%$ & 8 & $3.7 \%$ & 1 & $2.6 \%$ \\
\hline Moderate & 628 & $41.3 \%$ & 72 & $33.5 \%$ & 8 & $21.1 \%$ \\
\hline Poor & 533 & $35.0 \%$ & 72 & $33.5 \%$ & 13 & $34.2 \%$ \\
\hline Unknown & 309 & $20.3 \%$ & 63 & $29.3 \%$ & 16 & $42.1 \%$ \\
\hline
\end{tabular}


Table 1. Continued

\begin{tabular}{|c|c|c|c|c|c|c|}
\hline \multirow[t]{2}{*}{ Characteristics } & \multicolumn{2}{|c|}{ All } & \multicolumn{2}{|c|}{$\begin{array}{l}\text { Omission from } \\
\text { surgical resection }\end{array}$} & \multicolumn{2}{|c|}{$\begin{array}{c}\text { Deceased during } \\
\text { nCRT }^{*}\end{array}$} \\
\hline & $\mathrm{n}=1521$ & $(\%)$ & $\mathrm{n}=215$ & $(\%)$ & $\mathrm{n}=38$ & $(\%)$ \\
\hline \multicolumn{7}{|l|}{ Clinical T status } \\
\hline $\mathrm{T} 1$ & 22 & $1.4 \%$ & 3 & $1.4 \%$ & 0 & $0.0 \%$ \\
\hline $\mathrm{T} 2$ & 458 & $30.1 \%$ & 52 & $24.2 \%$ & 11 & $28.9 \%$ \\
\hline $\mathrm{T} 3$ & 981 & $64.5 \%$ & 145 & $67.4 \%$ & 25 & $65.8 \%$ \\
\hline $\mathrm{T} 4$ & 22 & $1.4 \%$ & 6 & $2.8 \%$ & 1 & $2.6 \%$ \\
\hline Unknown & 38 & $2.5 \%$ & 9 & $4.2 \%$ & 1 & $2.6 \%$ \\
\hline \multicolumn{7}{|l|}{ Clinical N status ${ }^{*}$} \\
\hline No & 602 & $39.6 \%$ & 73 & $34.0 \%$ & 7 & $18.4 \%$ \\
\hline N1 & 575 & $37.8 \%$ & 76 & $35.3 \%$ & 16 & $42.1 \%$ \\
\hline $\mathrm{N} 2$ & 282 & $18.5 \%$ & 52 & $24.2 \%$ & 12 & $31.6 \%$ \\
\hline N3 & 40 & $2.6 \%$ & 10 & $4.7 \%$ & 3 & $7.9 \%$ \\
\hline Unknown & 22 & $1.4 \%$ & 4 & $1.9 \%$ & 0 & $0.0 \%$ \\
\hline \multicolumn{7}{|l|}{ Year of diagnosis } \\
\hline 2015 & 732 & $48.1 \%$ & 79 & $36.7 \%$ & 14 & $36.8 \%$ \\
\hline 2016 & 789 & $51.9 \%$ & 136 & $63.3 \%$ & 24 & $63.2 \%$ \\
\hline $\begin{array}{l}\text { Exceeded original CROSS eligibility } \\
\text { criteria }{ }^{\S}\end{array}$ & 457 & $30.0 \%$ & 82 & $38.1 \%$ & 13 & $34.2 \%$ \\
\hline \multicolumn{7}{|c|}{$\begin{array}{l}\text { BMI body mass index at diagnosis; } n C R T \text { neoadjuvant chemoradiotherapy; WHO World Health Organization } \\
\text { * Please note: the patients who deceased during neoadjuvant chemoradiotherapy are also included in the patients } \\
\text { who did not undergo surgical resection. } \\
\text { † Closely related comorbidities were considered as one category. } \\
\text { * Clinical T status and N status are based on UICC TNM } 7 \text { th edition. } \\
{ }^{\circ} \text { CROSS eligibility criteria were defined as age } 18-74 \text { years, clinical T status } \leq \mathrm{T} 3 \text {, no history of other cancer, } \\
\text { tumor length } \leq 8 \mathrm{~cm} \text { and WHO } \leq 2 \text { (Note: all patients in this cohort were } \mathrm{cM} 0 \text { ). }\end{array}$} \\
\hline
\end{tabular}

status $(n=22)$, tumor length $>8 \mathrm{~cm}(\mathrm{n}=125)$, history of other cancer $(\mathrm{n}=169)$ and WHO performance status of $>2(n=1)$.

Patients in the cohort who underwent a surgical resection of the primary tumor, underwent this resection at a median of 62 days after the last day of nCRT (interquartile range 52-77 days).

\section{Omission from surgical resection}

A total of 215 patients (14.1\%) did not undergo surgical resection after starting nCRT. These patients had a mean age of $67.6(\mathrm{SD} \pm 9.4)$ years and mostly a moderate to poorly differentiated adenocarcinoma of the distal esophagus. Furthermore, 38.1\% (82/215) of these patients exceeded the original eligibility criteria of the CROSS trial.

Multivariable logistic regression analysis with omission from surgical resection as outcome identified age (pooled odds ratio [OR]: 1.04, 95\% CI: 1.01-1.06), BMI (pooled OR: 0.95, 
95\% CI: 0.90-0.99), WHO performance status (WHO 1: pooled OR: 1.62, 95\% CI: 1.16-2.62 and WHO 2: pooled OR: 3.53, 95\% CI: 1.68-7.41) and clinical N2 or N3 status (pooled OR: 1.57, 95\% CI: $1.04-2.37$ and pooled OR: 2.52 , 95\% CI: $1.14-5.55$, respectively) to be significantly associated with omission from surgical resection (Table 2). Exceeding the original eligibility criteria of the CROSS trial was not independently associated with a higher odds for omission of surgery (pooled OR: 0.99, 95\% CI: 0.70-1.40).

Table 2. Multivariable logistic regression analyses with omission from surgery as outcome variable

\begin{tabular}{|c|c|c|c|c|}
\hline \multirow[t]{2}{*}{ Predictors } & \multicolumn{2}{|c|}{$\begin{array}{c}\text { Complete case analysis } \\
\qquad(\mathrm{n}=833)\end{array}$} & \multicolumn{2}{|c|}{$\begin{array}{l}\text { Multiple imputation } \\
\text { (30 datasets) }\end{array}$} \\
\hline & Odds ratio & 95\% CI & Odds ratio & 95\% CI \\
\hline Age, years & 1.02 & $0.99-1.05$ & 1.04 & $1.01-1.06$ \\
\hline \multicolumn{5}{|l|}{ Sex } \\
\hline Male & reference & & reference & \\
\hline Female & 1.07 & $0.60-1.86$ & 0.81 & $0.55-1.20$ \\
\hline BMI, $\mathrm{kg} / \mathrm{m}^{2}$ & 0.95 & $0.90-1.00$ & 0.95 & $0.90-0.99$ \\
\hline \multicolumn{5}{|l|}{ WHO performance status } \\
\hline 0 & reference & & reference & \\
\hline 1 & 1.83 & $1.15-2.89$ & 1.62 & $1.16-2.62$ \\
\hline 2 & 3.54 & $1.05-10.46$ & 3.53 & $1.68-7.41$ \\
\hline \multicolumn{5}{|l|}{ Comorbidity categories $\dagger$} \\
\hline None & reference & & reference & \\
\hline One category & 0.73 & $0.40-1.31$ & 0.91 & $0.59-1.41$ \\
\hline Two or more categories & 0.88 & $0.50-1.57$ & 1.39 & $0.90-2.16$ \\
\hline \multicolumn{5}{|l|}{ Histology } \\
\hline Adenocarcinoma & reference & & reference & \\
\hline Squamous cell carcinoma & 1.38 & $0.79-2.37$ & 1.28 & $0.87-1.87$ \\
\hline \multicolumn{5}{|l|}{ Clinical T status ${ }^{*}$} \\
\hline $\mathrm{T} 1-\mathrm{T} 2$ & reference & & reference & \\
\hline $\mathrm{T} 3-\mathrm{T} 4$ & 1.50 & $0.87-2.68$ & 1.21 & $0.85-1.72$ \\
\hline \multicolumn{5}{|l|}{ Clinical N status } \\
\hline No & reference & & reference & \\
\hline N1 & 1.63 & $0.94-2.87$ & 1.07 & $0.75-1.54$ \\
\hline N2 & 2.92 & $1.60-5.40$ & 1.57 & $1.04-2.37$ \\
\hline N3 & 4.85 & $1.56-13.68$ & 2.52 & $1.14-5.55$ \\
\hline Exceeded original CROSS eligibility criteria ${ }^{\natural}$ & 1.17 & $0.71-1.91$ & 0.99 & $0.70-1.40$ \\
\hline
\end{tabular}

$B M I$ body mass index at diagnosis; WHO World Health Organization

${ }^{\dagger}$ Closely related comorbidities were considered as one category.

${ }^{*}$ Clinical $\mathrm{T}$ status and $\mathrm{N}$ status are based on UICC TNM 7th edition.

${ }^{\varsigma}$ CROSS eligibility criteria were defined as age 18-74 years, cT status $\leq \mathrm{T} 3$, no history of other cancer, tumor length $\leq 8 \mathrm{~cm}$ and $\mathrm{WHO} \leq 2$ (Note: all patients in this cohort were $\mathrm{cM} 0$ ).

Note. All variables included in this table were used for multiple imputation of the missing variables, as well as tumor location, tumor differentiation and the outcome. One patient with a WHO performance of 3 was excluded from the complete case analysis as well as the multiple imputation analysis. Bold values reflect significant odds ratio's. The intercept of the model of the complete case analysis was -3.28 and for the model after multiple imputation -3.46. 
The most frequently reported reasons for not proceeding to surgical resection in 2015 were disease progression $(43.0 \%, \mathrm{n}=34)$, physical functioning $(22.8 \%, \mathrm{n}=18)$ and patient preference $(16.5 \%, \mathrm{n}=13)$ (Table 3$)$.

\section{Mortality during neoadjuvant therapy}

A total of 38 patients $(2.5 \%)$ deceased during nCRT or the subsequent waiting period to surgery. The majority of the deceased patients were male $(89.5 \%)$, with a mean age of 68.0 $(\mathrm{SD} \pm 8.3)$ years. Most deceased patients had a WHO performance status of $1(39.5 \%$, $15 / 38$ ), comorbidities in $\geq 2$ categories (57.9\%, 22/38), of which cardiovascular comorbidities and hypertension were the most frequently present (50.0\% [19/38] and 44.7\% [17/38], respectively). Furthermore, $81.6 \%$ (31/38) of patients had at least 1 lymph node metastasis at clinical staging (clinical N1-3). Among these deceased patients, 34.2\% ( $\mathrm{n}=13)$ exceeded the original eligibility criteria of the CROSS trial. A detailed description of these patient demographics is included in Table 1. The patients who deceased during nCRT are also included in the patients who did not undergo surgical resection.

Table 3. Reasons for omission from surgery in patients who started neoadjuvant chemoradiotherapy (nCRT) for esophageal cancer in 2015

\begin{tabular}{|c|c|c|}
\hline \multirow[t]{2}{*}{ Reasons } & \multirow[b]{2}{*}{$\mathrm{n}$} & \multirow[b]{2}{*}{$(\%)$} \\
\hline & & \\
\hline $\begin{array}{l}\text { All patients who started nCRT but did not proceed } \\
\text { to surgery in } 2015\end{array}$ & 79 & $100 \%$ \\
\hline Disease progression & 34 & $43.0 \%$ \\
\hline Impaired physical functioning & 18 & $22.8 \%$ \\
\hline Patient preference & 13 & $16.5 \%$ \\
\hline Comorbidities & 2 & $2.5 \%$ \\
\hline Complications or toxicity of previous therapy & 3 & $3.8 \%$ \\
\hline Low tumor load & 1 & $1.3 \%$ \\
\hline Other/unknown & 8 & $10.1 \%$ \\
\hline $\begin{array}{l}\text { Subgroup of patients who did not proceed to surgery and deceased } \\
\text { during nCRT or subsequent waiting period to surgery in } 2015\end{array}$ & 15 & $100 \%$ \\
\hline Disease progression & 4 & $26.7 \%$ \\
\hline Impaired physical functioning & 3 & $20.0 \%$ \\
\hline Patient preference & 1 & $6.7 \%$ \\
\hline Comorbidities & 1 & $6.7 \%$ \\
\hline Complications or toxicity of previous therapy & 2 & $13.3 \%$ \\
\hline Low tumor load & 0 & $0.0 \%$ \\
\hline Other/unknown & 4 & $26.7 \%$ \\
\hline
\end{tabular}




\section{Survival}

Kaplan-Meier survival curves for 1-year overall survival of all patients with esophageal cancer that started nCRT per treatment group (i.e. nCRT followed by surgery and nCRT without surgery) are depicted in Figure 1. No patients were censored, as follow-up time included at least 365 days from start of nCRT for all patients. One-year overall survival of the patients who underwent nCRT followed by surgical resection was $94.9 \%$. One-year overall survival of the patients who did not undergo surgical resection was $73.5 \%$.

1-Year Overall Survival

All patients who started $\mathrm{nCRT}$

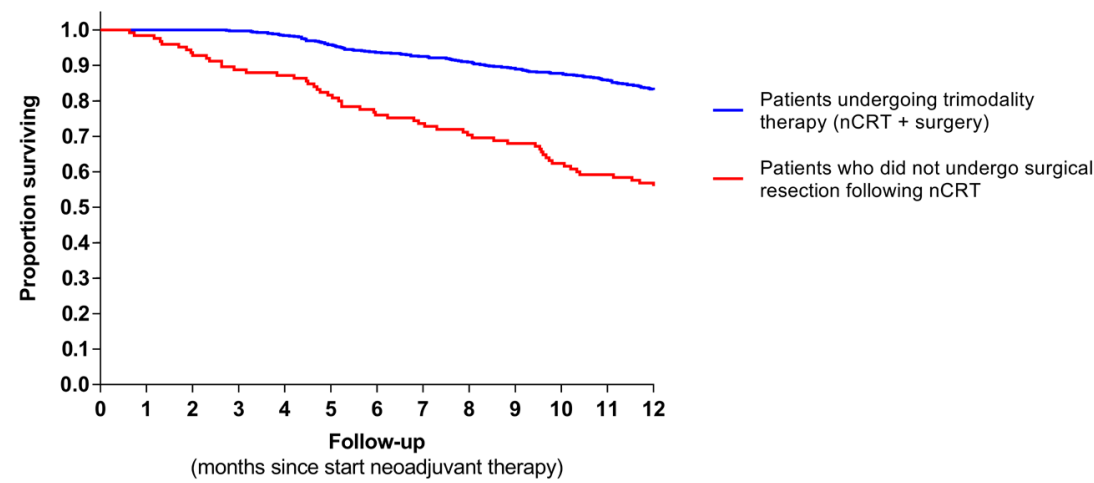

\begin{tabular}{|llllll|}
\hline Number at risk & & & & & \\
nCRT + surgery & 697 & 696 & 654 & 622 & 581 \\
nCRT without surgery & 125 & 112 & 96 & 86 & 71 \\
\hline
\end{tabular}

Figure 1. Kaplan-Meier estimates of 1-year overall survival for patients with esophageal cancer that started neoadjuvant chemoradiotherapy (nCRT) in the Netherlands in 2015 and 2016. Please note that the categorization of patients by treatment, results in immortal time bias from the start of neoadjuvant therapy until the date of surgery for patients who underwent nCRT followed by surgery (blue curve).

\section{Discussion}

This population-based observational cohort study aimed to gain insight in unplanned omission from surgical resection after starting nCRT for esophageal cancer, arising from patient preference and disease progression as reasons for not proceeding to surgical resection, as well as mortality during nCRT. It demonstrated that 1 in 7 (14.1\%) patients did not undergo the planned surgical resection and 1 in $40(2.5 \%)$ of patients who started nCRT for esophageal cancer deceased during nCRT or in the subsequent waiting period to surgery. 
Surgery following nCRT in patients with locally advanced esophageal cancer is associated with significantly improved survival compared to chemoradiotherapy alone (i.e. definitive CRT), especially for esophageal adenocarcinoma. ${ }^{20,21}$ Furthermore, salvage surgery appears to be associated with poorer short-term outcomes in patients with persistent or recurrent disease after definitive CRT, when compared to planned esophagectomy following nCRT. ${ }^{22}$ As such, curative treatment should aim to include surgical resection and patients and multidisciplinary tumor boards should be made aware of these differences in outcomes. ${ }^{22}$ However, in our cohort, $14.1 \%$ of patients who were deemed eligible for nCRT followed by esophagectomy did not undergo surgical resection, which is higher than the percentage reported in the randomized controlled CROSS trial (7.6\%). The main reason for not undergoing surgical resection in the cohort from 2015 was disease progression. Disease progression, including systemic interval metastases, was reported as reason for not undergoing surgical resection in $4.8 \%$ of the entire cohort from 2015 (35/732). Contrarily, studies reporting on the value of a standard restaging PET-CT after nCRT in detecting interval metastases observed interval metastases in $8 \%$ of cases. ${ }^{23,24}$ The discrepancy between these results and the results obtained in the present study is most likely to be explained by the fact that a restaging PET-CT was not routinely performed in the Netherlands in 2015. As such, interval metastases could have remained unnoticed and the $4.8 \%$ disease progression found in our study is most likely under-reported. Disease progression as reason for omission from surgical resection might furthermore be reflected in the finding that clinical N2 or N3 status was significantly associated with a higher odds of omission of surgical resection, as it has been described that positive $\mathrm{N}$ status at diagnosis increases the probability of developing interval metastases. ${ }^{24}$

Physical functioning, the reduction thereof reported as a reason for not undergoing surgical resection in $22.8 \%$ of patients who did not undergo surgical resection in 2015 , could be increased by structured counseling by a multidisciplinary team of physical therapists and dieticians. ${ }^{25}$ The time between diagnosis and nCRT, as well as the interval between nCRT and surgical resection, is highly suitable for interventions to stimulate a patients physical functioning. This might subsequently allow these patients to undergo surgical resection after all.

Patient preference was reported as the reason for not undergoing surgical resection in $16.5 \%$ of these patients, which underlines the importance of counseling and shared decision making. Besides survival, quality of life is highly relevant to patients in treatment decisionmaking. ${ }^{26}$ As esophagectomy has a lasting impact on quality of life, this might be another reason for patients to opt for not undergoing surgical resection. ${ }^{27,28}$ A shared decisionmaking tool, which aims to transparently discuss both the benefits and drawbacks from all treatment strategies including survival and quality of life between the patient and their physician, might further aid informed shared decision-making. 
This patient preference might furthermore be driven by increasing attention for patients with a clinical complete response after nCRT, in whom an active surveillance approach might be feasible. ${ }^{29}$ However, to date survival is significantly better for patients with a clinical complete response to nCRT who underwent surgery compared to active surveillance. ${ }^{21,30}$ This is most likely influenced by the lack of reliable diagnostic tests to accurately select patients with a pathological complete response. As such, it is felt that an accurate model should first be developed, before pursuing an active surveillance approach in clinical practice. Given the patient selection criteria that are employed in randomized controlled trials, the applicability of nCRT to all patients with esophageal cancer might be questionable. ${ }^{12}$ The results of the present study show that the eligibility criteria for nCRT in clinical practice have been extended in one third of the patients, which might have led to a higher mortality rate during $\mathrm{nCRT}$ or in the subsequent waiting period to surgery than in the randomized controlled CROSS trial (2.5\% versus $0.6 \%$, respectively). Previous single center studies however have demonstrated that extension of the CROSS eligibility criteria for nCRT did not affect nCRT associated toxicity or survival. ${ }^{31,32}$ The difference between the single center studies and the current population-based study might be explained by hospital variation, as hospital variation is a known important factor to influence treatment for esophageal cancer. ${ }^{33}$ As such, hospital variation might also influence the support vulnerable patients who undergo nCRT receive. Exceeding the original eligibility criteria of the CROSS trial was not significantly associated with unplanned omission from surgical resection in the current cohort, even though the patients who did not undergo surgical resection following nCRT was double that of the CROSS trial. This difference might not only explained by the selection bias caused by employing strict inclusion criteria, but also by the selection bias of patients who are willing to participate in a randomized controlled trial. Furthermore, no data was available in the registry to account for weight loss, previous radiotherapy or chemotherapy as well as hematologic, renal, hepatic, and pulmonary function, which are the remaining eligibility criteria of the CROSS trial. These patient-related factors might also have contributed to more patients who did not proceed to surgical resection in the current study, but could not be accounted for.

Our results show age, BMI at diagnosis and performance status to be associated with unplanned omission from surgery. It is important to realize that our descriptive study merely describes current clinical practice. Hence, no causal conclusions can be drawn whether older patients or patients with an impaired performance status should undergo nCRT before esophagectomy based on the current data, nor based on the (conflicting) results reported by previous literature on this matter. ${ }^{34-39}$ Overall, advanced age should not be considered as a strict contraindication to either nCRT or esophagectomy. Whether or not nCRT should function as selection mechanism for patients who are eligible for surgery, or patients should be selected for nCRT remains unresolved. Nevertheless, even though factors like age and performance status are not modifiable, our results could aid physicians 
in the identification of patients who might benefit from increased counseling in the preoperative trajectory, i.e. patients with higher age, lower BMI and impaired performance status. These patients could be offered supervised prehabilitation for surgery, a preoperative geriatric assessment to obtain a holistic view of the patient or more frequent follow-up visits with their own oncologist or surgeon to motivate them and their caregivers for surgery. Insights into these factors will help treating clinicians and their patients predict the balance of harms and benefit and may ultimately improve clinical decision making.

Baseline differences between patient groups are furthermore likely to influence the observed differences in the 1-year overall survival curves for the different treatment groups (as depicted in Figure 1). Since the study data is obtained from routine clinical practice, confounding by indication as form of selection bias for these treatment strategies will likely influence the observed results. Therefore, these results warrant careful interpretation and no statistical analyses were performed for this comparison.

The population-based design with virtually complete inclusion of all eligible patients in the Netherlands is a significant strength of the study, along with the fact that we had detailed information on the nCRT regimen and all patients underwent the same regimen. It should be noted that there are also limitations associated with population-based observational studies such as this, for instance the lack of knowledge on the cause of death and hospital information.

In summary, mortality during nCRT for esophageal cancer is considerable. Furthermore, 1 in 7 patients who started nCRT eventually does not proceed to surgical resection. These patients have a lower overall survival compared to the patients who undergo nCRT followed by surgical resection. This could be a starting point for further research, which should aim to investigate whether this patient group can be selected prior to treatment, and whether patient-individualized treatment, centralization of oncological care and counseling will result in a larger proportion of patients who will undergo surgery. 


\section{References}

1. Busweiler LAD, Wijnhoven BPL, van Berge Henegouwen MI, et al. Early outcomes from the Dutch Upper Gastrointestinal Cancer Audit. Br J Surg. 2016;103(13):1855-1863.

2. Brusselaers N, Mattsson F, Lagergren J. Hospital and surgeon volume in relation to long-term survival after oesophagectomy: systematic review and meta-analysis. Gut. 2014;63(9):1393-1400.

3. Coupland VH, Lagergren J, Lüchtenborg $\mathrm{M}$, et al. Hospital volume, proportion resected and mortality from oesophageal and gastric cancer: a population-based study in England, 2004-2008. Gut. 2013;62(7):961966.

4. Derogar M, Sadr-Azodi O, Johar A, Lagergren P, Lagergren J. Hospital and Surgeon Volume in Relation to Survival After Esophageal Cancer Surgery in a Population-Based Study. J Clin Oncol. 2013;31(5):551-557.

5. Anderson $\mathrm{O}, \mathrm{Ni} \mathrm{Z}$, Møller $\mathrm{H}$, et al. Hospital volume and survival in oesophagectomy and gastrectomy for cancer. Eur J Cancer. 2011;47(16):2408-2414.

6. Findlay JM, Gillies RS, Millo J, Sgromo B, Marshall RE, Maynard ND. Enhanced recovery for esophagectomy: a systematic review and evidence-based guidelines. Ann Surg. 2014;259(3):413-431.

7. Markar SR, Schmidt H, Kunz S, Bodnar A, Hubka M, Low DE. Evolution of standardized clinical pathways: refining multidisciplinary care and process to improve outcomes of the surgical treatment of esophageal cancer. J Gastrointest Surg. 2014;18(7):1238-1246.

8. van Hagen P, Hulshof MCCM, van Lanschot JJB, et al. Preoperative Chemoradiotherapy for Esophageal or Junctional Cancer. N Engl J Med. 2012;366(22):2074-2084.

9. Shapiro J, Lanschot JJB Van, Hulshof MCCM, et al. Neoadjuvant chemoradiotherapy plus surgery versus surgery alone for oesophageal or junctional cancer (CROSS): long-term results of a randomised controlled trial. Lancet Oncol. 2015;16(9):1090-1098.

10. Sjoquist KM, Burmeister BH, Smithers BM, et al. Survival after neoadjuvant chemotherapy or chemoradiotherapy for resectable oesophageal carcinoma: an updated meta-analysis. Lancet Oncol. 2011;12(7):681-692.

11. Mariette C, Dahan L, Mornex F, et al. Surgery alone versus chemoradiotherapy followed by surgery for stage I and II esophageal cancer: final analysis of randomized controlled phase III trial FFCD 9901. J Clin Oncol. 2014;32(23):2416-2422.

12. Markar SR, Johar A, Maisey N, Lagergren P, Lagergren J. Complications during neoadjuvant therapy and prognosis following surgery for esophageal cancer. Dis Esophagus. 2018;31(5).

13. Robb WB, Messager M, Gronnier C, et al. High-Grade Toxicity to Neoadjuvant Treatment for Upper Gastrointestinal Carcinomas: What is the Impact on Perioperative and Oncologic Outcomes? Ann Surg Oncol. 2015;22(11):3632-3639.

14. Mariette C, Brouquet A, Tzanis D, et al. What is the impact of neoadjuvant chemoradiation on outcomes in gastro-intestinal cancer? J Visc Surg. 2017;154:185-195.

15. Kennedy-Martin T, Curtis S, Faries D, Robinson S, Johnston J. A literature review on the representativeness of randomized controlled trial samples and implications for the external validity of trial results. Trials. $2015 ; 16$.

16. National guideline esophageal cancer (version 3.1). 2015. http://www.oncoline.nl/oesofaguscarcinoom. Accessed March 21, 2018.

17. Washington K. 7th Edition of the AJCC Cancer Staging Manual: Stomach. Ann Surg Oncol. 2010;17(12):30773079 .

18. Sterne JAC, White IR, Carlin JB, et al. Multiple imputation for missing data in epidemiological and clinical research: potential and pitfalls. BMJ. 2009;338:b2393.

19. White IR, Royston P, Wood AM. Multiple imputation using chained equations: Issues and guidance for practice. Stat Med. 2011;30(4):377-399.

20. Hategan M, Cook N, Prewett S, Hindmarsh A, Qian W, Gilligan D. Trimodality therapy and definitive chemoradiotherapy for esophageal cancer: a single-center experience and review of the literature. Dis Esophagus. 2015;28(7):612-618.

21. Barbetta A, Hsu M, Tan KS, et al. Definitive chemoradiotherapy versus neoadjuvant chemoradiotherapy followed by surgery for stage II to III esophageal squamous cell carcinoma. J Thorac Cardiovasc Surg. 2018;155(6):2710-2721.e3. 
22. Markar SR, Karthikesalingam A, Penna M, Low DE. Assessment of Short-Term Clinical Outcomes following Salvage Esophagectomy for the Treatment of Esophageal Malignancy: Systematic Review and Pooled Analysis. Ann Surg Oncol. 2014;21(3):922-931.

23. Stiekema J, Vermeulen D, Vegt E, et al. Detecting Interval Metastases and Response Assessment Using 18F-FDG PET/CT After Neoadjuvant Chemoradiotherapy for Esophageal Cancer. Clin Nucl Med. 2014;39(10):862-867.

24. Goense L, Ruurda JP, Carter BW, et al. Prediction and diagnosis of interval metastasis after neoadjuvant chemoradiotherapy for oesophageal cancer using 18F-FDG PET/CT. Eur J Nucl Med Mol Imaging. 2018;45(10):1742-1751.

25. Borggreve ASAS, Kingma BFF, Domrachev SASA, et al. Surgical treatment of esophageal cancer in the era of multimodality management. Ann N Y Acad Sci. 2018;1434(1):192-209.

26. Noordman BJ, de Bekker-Grob EW, Coene PPLO, et al. Patients' preferences for treatment after neoadjuvant chemoradiotherapy for oesophageal cancer. Br J Surg. 2018;105(12):1630-1638.

27. Kauppila JH, Johar A, Lagergren P. Postoperative Complications and Health-related Quality of Life 10 Years After Esophageal Cancer Surgery. Ann Surg. July 2018.

28. Noordman BJ, Verdam MGE, Lagarde SM, et al. Impact of Neoadjuvant Chemoradiotherapy on Health Related Quality of Life In Long-Term Survivors of Esophageal or Junctional Cancer: Results from the Randomized Cross Trial. Ann Oncol. 2018;29(2):445-451.

29. Noordman BJ, Wijnhoven BPL, Lagarde SM, et al. Active surveillance in clinically complete responders after neoadjuvant chemoradiotherapy for esophageal or junctional cancer. Dis Esophagus. 2017;30(12):1-8.

30. Piessen G, Messager M, Mirabel X, et al. Is there a role for surgery for patients with a complete clinical response after chemoradiation for esophageal cancer? An intention-to-treat case-control study. Ann Surg. 2013;258(5):793-9-800.

31. de Heer EC, Hulshoff JB, Klerk D, et al. Effect of Extending the Original Eligibility Criteria for the CROSS Neoadjuvant Chemoradiotherapy on Toxicity and Survival in Esophageal Cancer. Ann Surg Oncol. 2017;24(7):1811-1820.

32. Toxopeus E, van der Schaaf M, van Lanschot J, et al. Outcome of Patients Treated Within and Outside a Randomized Clinical Trial on Neoadjuvant Chemoradiotherapy Plus Surgery for Esophageal Cancer: Extrapolation of a Randomized Clinical Trial (CROSS). Ann Surg Oncol. 2018;25(8):2441-2448.

33. van Putten M, Koëter M, van Laarhoven HWM, et al. Hospital of Diagnosis Influences the Probability of Receiving Curative Treatment for Esophageal Cancer. Ann Surg. 2018;267(2):303-310.

34. Lagarde SM, Reitsma JB, Maris A-KD, et al. Preoperative Prediction of the Occurrence and Severity of Complications After Esophagectomy for Cancer With Use of a Nomogram. Ann Thorac Surg. 2008;85:19381946.

35. Ruol A, Portale G, Castoro C, et al. Effects of Neoadjuvant Therapy on Perioperative Morbidity in Elderly Patients Undergoing Esophagectomy for Esophageal Cancer. Ann Surg Oncol. 2007;14(11):3243-3250.

36. Paulus E, Ripat C, Koshenkov V, et al. Esophagectomy for cancer in octogenarians: should we do it? Langenbeck's Arch Surg. 2017;402(3):539-545.

37. Vlacich G, Samson PP, Perkins SM, et al. Treatment utilization and outcomes in elderly patients with locally advanced esophageal carcinoma: a review of the National Cancer Database. Cancer Med. 2017;6(12):28862896.

38. Fogh SE, Yu A, Kubicek GJ, et al. Do Elderly Patients Experience Increased Perioperative or Postoperative Morbidity or Mortality When Given Neoadjuvant Chemoradiation Before Esophagectomy? Int J Radiat Oncol. 2011;80(5):1372-1376.

39. Nienhueser H, Kunzmann R, Sisic L, et al. Surgery of gastric cancer and esophageal cancer: Does age matter? J Surg Oncol. 2015;112(4):387-395. 


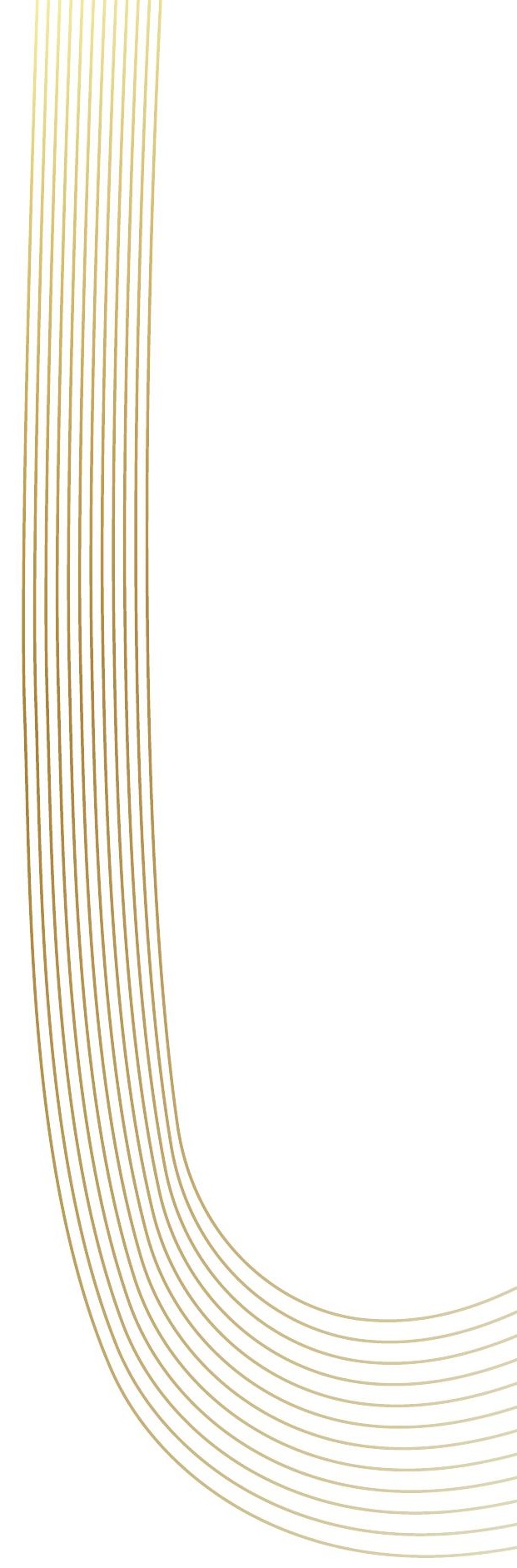


Chapter 11

Generalized cardiovascular disease on a preoperative CT scan is predictive for anastomotic leakage after esophagectomy

European Journal of Surgical Oncology 2018;44(5):587-593.

A.S. Borggreve*, L. Goense ${ }^{\star}$, P.S.N. van Rossum, R. van Hillegersberg, P.A. de Jong, J.P. Ruurda

${ }^{*}$ Joint first authorship. 


\begin{abstract}

\section{Background}

Recent studies demonstrated that calcification of arteries supplying the gastric tube is associated with anastomotic leakage after esophagectomy. However, it remains unclear whether this association only derives from local flow limitations, or generalized vascular disease as well. The purpose of this study was to determine whether calcification throughout the entire cardiovascular system is associated with anastomotic leakage.
\end{abstract}

\title{
Methods
}

Consecutive patients who underwent an esophagectomy with gastric tube reconstruction and cervical anastomosis for esophageal cancer were analyzed. Diagnostic CT images were scored for the presence of arterial calcification on 10 locations based on a visual grading system. The association with anastomotic leakage was studied using logistic regression analysis.

\section{Results}

A total of 406 patients were included for analysis of whom 104 developed anastomotic leakage (25.6\%). Presence of calcification in the coronary arteries (minor calcification: $36.5 \%$ leakage; no calcification: $18.1 \%, p=.001$ ), supra-aortic arteries (minor calcification: $30.9 \%$ leakage; major calcification: $35.3 \%$; no calcification: $16.1 \%, p=.007$ and $p<.001$, respectively) and thoracic aorta (major calcification: $33.3 \%$ leakage; no calcification: $19.4 \%$, $p=.011)$ was associated with leakage. In multivariable analysis, minor calcification of the coronary arteries (OR 2.29, 95\% CI: 1.28-4.12, $p=.005$ ) and calcification of the supra-aortic arteries (OR 2.48, 95\% CI: 1.30-4.74, $p=.006$ for minor calcification and OR 2.72, 95\% CI: $1.49-4.99, p=.001$ for major calcification) remained independently associated with leakage.

\section{Conclusions}

Calcification of the coronary and supra-aortic arteries on routine CT are predictive of cervical anastomotic leakage after esophagectomy. These results suggest that generalized cardiovascular disease is a strong indicator for the risk of leakage. 


\section{Introduction}

Anastomotic leakage after esophagectomy for patients with esophageal carcinoma is a frequently encountered complication (10-30\%), resulting in increased postoperative morbidity and mortality. ${ }^{1-9}$ Several studies have aimed to identify preoperative risk factors for anastomotic leakage after esophagectomy. ${ }^{2,10,11}$ Patient-related factors associated with anastomotic leakage include obesity, heart failure, coronary artery disease, peripheral vascular disease, hypertension, steroids, diabetes, renal insufficiency and tobacco use. ${ }^{2}$ The majority of these risk factors underline the current hypothesis that ischemia is one of the most important contributors to anastomotic leakage, since most of these factors negatively influence microvascular perfusion and thus compromise anastomotic healing. ${ }^{2,4}$ Preoperative identification of patients with esophageal cancer at high risk of anastomotic leakage may provide opportunities to modify these risk factors or more fully optimize patients to reduce their risk of anastomotic leakage. However, predicting anastomotic leakage based only on standard patient-related risk factors remains challenging, encouraging further research into prediction strategies. ${ }^{11}$

Radiographic findings such as atherosclerotic calcification on a computed tomography (CT) scan can be used to objectively evaluate a patients' vascular status and risk of cardiovascular events. ${ }^{12-14}$ Recent studies demonstrated that atherosclerotic calcification of the thoracic aorta and right postceliac arteries is associated with anastomotic leakage after esophagectomy. ${ }^{11,15}$ To date, however, it remains unclear whether the association between anastomotic leakage and calcification detected on a CT scan applies to local vascular disease (with accompanied local flow limitations) only, or to generalized vascular disease as well. Therefore, the purpose of this study was to determine whether the presence of atherosclerotic calcification throughout the entire cardiovascular system as determined on routine CT images is associated with anastomotic leakage after esophagectomy for cancer.

\section{Patients and Methods}

This study was approved by the Medical Ethics Committee of the University Medical Center Utrecht (number 15/624); the requirement for written informed consent was waived.

\section{Study population}

All consecutive patients who underwent elective esophagectomy for cancer with gastric tube reconstruction and handsewn cervical anastomosis from October 2003 to October 2015 at the University Medical Center Utrecht were considered for inclusion. Exclusion criteria included premature discontinuation of surgery due to the discovery of T4b or M1 disease during surgery, combined laryngeal resection, salvage surgery and insufficient quality of CT scan. 
Patient and treatment-related characteristics, and surgical outcome data (e.g. anastomotic leakage), were collected from a prospectively maintained database. Anastomotic leakage was defined by either visible loss of saliva through the cervical wound, extravasation of water-soluble contrast material during a contrast swallow study or CT scan, or visualization of anastomotic dehiscence or fistulae during endoscopy or surgical re-intervention.

\section{Image acquisition}

CT images of the neck, thorax and (upper) abdomen were routinely conducted during diagnostic workup with multidetector row CT scanners from various vendors at our own or referring institutions. Images were acquired with a tube potential varying from 100 to $140 \mathrm{kV}$, a minimum tube current of 8-500 mAs (median $73 \mathrm{mAs}$ ), a maximum tube current of 33-500 mAs (median $181 \mathrm{mAs}$ ) and typically with a field-of-view of $500 \mathrm{~mm}$. The scans were typically contrast-enhanced $(90.1 \%)$. All routine preoperative CT protocols with a maximum slice thickness $\leq 7 \mathrm{~mm}$ were considered suitable for inclusion. Median slice thickness was $3.0 \mathrm{~mm}$ (range $0.9-7.0 \mathrm{~mm}$, IQR $4.0 \mathrm{~mm}$ ), with a slice thickness of $5.0 \mathrm{~mm}$ or less in $98.8 \%(401 / 406)$ of patients. The interval between CT scanning and primary surgery ranged from 1 to 280 days, with a median of 114 days.

\section{Image evaluation}

A detailed visual grading system was developed in order to consistently score CT images on arterial calcification at ten different locations (Table 1). The selected locations included the supra-aortic arteries (i.e. the brachiocephalic trunk, left common carotid artery and left subclavian artery), coronary arteries, aortic valve, thoracic aorta (with special attention to a possible calcified ductus arteriosus that was not scored as a calcification), abdominal aorta, celiac axis, common iliac arteries (left and right) and external iliac arteries (left and right). Scores of 0,1 or 2 were assigned, corresponding with absence, minor presence or major presence of calcification, respectively. Examples of arterial calcification on CT images are presented in Figure 1.

Images were typically analyzed in the transverse plane with software from Sectra: PACS IDS7 $^{\mathrm{TM}}$ version 17.3. All CT images were scored independently by one reader (A.S.B.), trained and supervised by a radiologist with 10 years of experience in thoracic and abdominal CT evaluation (P.A.d.J.). In addition, a random sample of 30 patients without missing data was scored twice by one reader (A.S.B.) after a 12-month interval between readings, as well as scored independently by a second reader (L.G.) to determine intra- and interobserver reproducibility and agreement, respectively. In previous studies, this type of grading calcification has been shown to yield good to excellent intra- and interobserver reproducibility and agreement. ${ }^{12,15,16}$ The readers were blinded for patient and treatmentrelated factors, and outcome in terms of anastomotic leakage. 


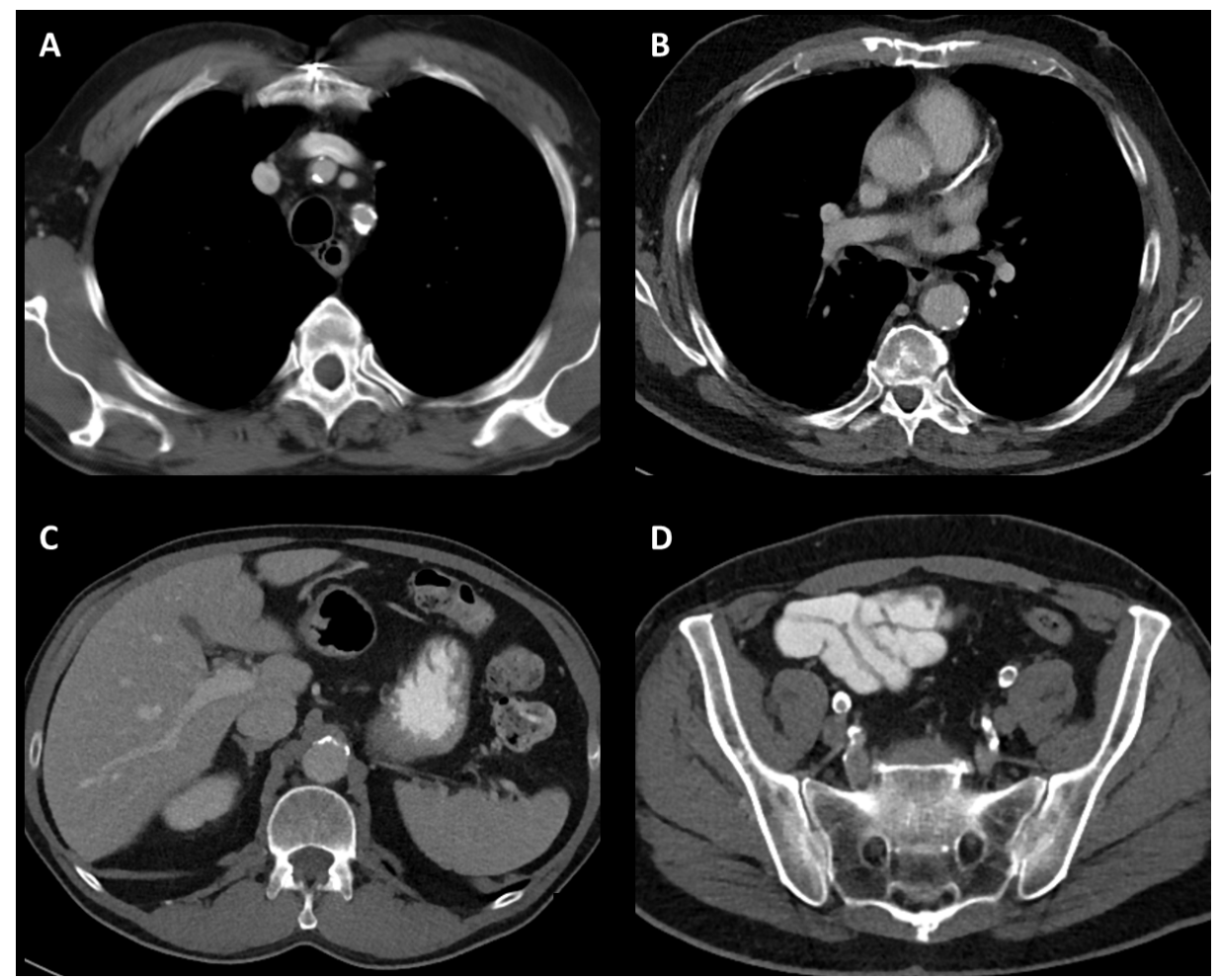

Figure 1. Examples of preoperative CT images of arterial calcification in patients with esophageal cancer.

A: Calcification of the brachiocephalic and left subclavian artery, resulting in a score of 2 for calcification of the supra-aortic arteries.

B: Calcification of a large segment of the left anterior descending artery, resulting in a score of 2 for calcification of coronary arteries.

C: Calcification of the celiac axis with a maximum cross-sectional diameter of $>10 \mathrm{~mm}$, resulting in a score of 2 for calcification of the celiac axis.

D: Multiple calcified foci of the internal and external iliac arteries, resulting in a score of 2 for calcification of the internal and external iliac arteries.

\section{Statistical analyses}

The association between patient and treatment-related characteristics and anastomotic leakage was studied univariably. Depending on the cell count, the $\chi^{2}$ or Fisher's exact test was used for categorical variables. The independent samples t-test or Mann-Whitney U test were used for normally or skewed distributed continuous variables, respectively.

The association between calcification scores and anastomotic leakage was studied per location in an univariable and multivariable logistic regression model. Variables to be entered into the multivariable logistic regression model along with the calcification score were based on clinical reasoning and literature review to be able to assess whether the calcification score 
Table 1. Definitions used to visually grade arterial calcification on preoperative CT images.

\begin{tabular}{|c|c|c|c|}
\hline \multirow[t]{2}{*}{ Anatomical location } & \multicolumn{3}{|c|}{ Calcification scores } \\
\hline & $\mathbf{0}$ & 1 & 2 \\
\hline Coronary arteries & absent & $\begin{array}{l}\text { multiple foci or } \\
1 \text { calcification extending } \\
\text { over } \geq 2 \text { slices }\end{array}$ & $\begin{array}{l}\text { calcified arteries covering a large } \\
\text { segment of a coronary branch }\end{array}$ \\
\hline Supra-aortic arteries & absent & $\begin{array}{l}\text { calcification in } 1 \text { supra-aortic } \\
\text { artery }\end{array}$ & $\begin{array}{l}\text { calcification in }>1 \text { supra-aortic } \\
\text { artery }\end{array}$ \\
\hline Aortic valve & absent & 1 small calcification on 1 leaflet & $>1$ small calcification on 1 leaflet \\
\hline $\begin{array}{l}\text { Thoracic aorta } \\
\text { (heart - celiac axis) }\end{array}$ & absent & $\begin{array}{l}\leq 9 \text { foci } \text { or } \\
\leq 3 \text { calcification extending } \\
\text { over } \geq 3 \text { slices }\end{array}$ & $\begin{array}{l}>9 \text { foci or } \\
>3 \text { calcification extending } \\
\text { over } \geq 3 \text { slices }\end{array}$ \\
\hline Celiac axis & absent & $\begin{array}{l}\text { single focus with MCSD } \leq 10 \mathrm{~mm} \\
\text { or } \\
\text { extending over }<3 \text { slices }\end{array}$ & $\begin{array}{l}\text { MCSD }>10 \text { mm or } \\
\text { extending over } \geq 3 \text { slices or } \\
\text { involving proximal (aortoceliac) } \\
\text { and distal (hepatosplenic) parts }\end{array}$ \\
\hline $\begin{array}{l}\text { Abdominal aorta } \\
\text { (celiac axis - bifurcation) }\end{array}$ & absent & $\begin{array}{l}\leq 9 \text { foci } \text { or } \\
\leq 3 \text { calcification extending } \\
\text { over } \geq 3 \text { slices }\end{array}$ & $\begin{array}{l}>9 \text { foci } \text { or } \\
>3 \text { calcification extending } \\
\text { over } \geq 3 \text { slices }\end{array}$ \\
\hline Common iliac arteries & absent & $\begin{array}{l}\leq 5 \text { foci or } \\
1 \text { calcification extending } \\
\text { over } \geq 3 \text { slices }\end{array}$ & $\begin{array}{l}>5 \text { foci } \text { or } \\
>1 \text { calcification extending } \\
\text { over } \geq 3 \text { slices }\end{array}$ \\
\hline External iliac arteries & absent & $\begin{array}{l}\leq 5 \text { foci or } \\
1 \text { calcification extending } \\
\text { over } \geq 3 \text { slices }\end{array}$ & $\begin{array}{l}>5 \text { foci } \text { or } \\
>1 \text { calcification extending } \\
\text { over } \geq 3 \text { slices }\end{array}$ \\
\hline
\end{tabular}

MCSD: maximum cross-sectional diameter.

was independently and significantly associated with the occurrence of anastomotic leakage. ${ }^{17,18}$ Odds ratios (ORs) with corresponding 95\% confidence intervals (CIs) and Wald test statistic p-values were calculated. Surgery-related factors were excluded from this model because this parameter would not be useful in preoperative risk assessment. ${ }^{19}$

Some CT scans did not include all regions of interest, so not all sites could be assessed for the presence of calcification $(1.2 \%$ [5/406], 6.7\% [27/406] and 9.1\% [37/406] of the CT scans for the abdominal aorta, common iliac arteries and the external iliac arteries, respectively). These missing data were considered missing at random (MAR). Multiple imputation of these missing sites was applied to replace these missing values for logistic regression analysis, using 20 imputed datasets. ${ }^{20,21}$

Reproducibility of the calcification scoring system within and between observers was assessed with reliability and agreement analyses. Overall proportions of agreement were 
calculated to determine the intra- and interobserver agreement of the calcification scoring model. Intra- and interobserver reliability for grading the calcification scores of all anatomical locations was assessed by Cohen's linearly weighted kappa $(\kappa) .{ }^{22}$ The weighted $\kappa$ statistic can be interpreted as follows: $\kappa$ of 0.81 to 1.00 indicates excellent reliability; $\kappa$ of 0.61 to 0.80 , good reliability; $\kappa$ of 0.41 to 0.60 , moderate reliability; $\kappa$ of 0.21 to 0.40 , fair reliability; and $\kappa$ of less than 0.20 , poor reliability. ${ }^{23}$

Statistical analysis was performed using SPSS 23.0 (IBM Corp., Armonk, NY). An online statistical tool was used to analyze reliability and agreement of the calcification scoring system (http://vassarstats.net/kappa.html). Probability values $<.05$ were considered to be statistically significant.

\section{Results}

A total of 497 patients underwent esophagectomy during the study period, of which 91 patients were excluded based because of benign disease $(n=18)$, non-elective surgery $(n$ $=7)$, reconstruction other than gastric tube $(n=7)$, intrathoracic anastomosis $(n=5)$ premature discontinuation of surgery due to the discovery of T4b or M1 disease during surgery $(n=10)$, combined laryngeal resection $(n=8)$, salvage surgery $(n=1)$ and insufficient quality of CT scan $(\mathrm{n}=34)$ (Figure 2). One patient experienced a major acute myocardial infarction within 3 days after esophagectomy and was excluded from further analysis.

The 406 patients that were included for analysis had a mean age of 64 years, and $73.9 \%$ (n $=300$ ) were male. In the majority of patients treatment consisted of neoadjuvant therapy (chemotherapy or concurrent chemoradiotherapy) followed by thoracolaparoscopic esophagectomy.

A total of 104 patients (25.6\%) experienced anastomotic leakage after a median of 7 days (range, 1 to 26 days) postoperatively. Patient and treatment-related characteristics and their univariable association with anastomotic leakage are presented in Table 2. Of these variables, $\operatorname{COPD}(p=.005)$ and a transhiatal surgical approach $(p=.024)$ were significantly associated with anastomotic leakage. Furthermore, patients with anastomotic leakage appeared slightly older (mean 65.5 versus 63.7 years, $p=.082$ ) and more frequently had a history of diabetes mellitus (19.2\% versus $12.3 \%, p=.077)$.

Distribution of arterial calcification among patients with and without anastomotic leakage is shown in Table 3. For all studied vascular locations, calcifications were more frequently observed in the anastomotic leakage group compared to the group without anastomotic leakage. Only 6\% (23/406) of patients did not have arterial calcifications. The absolute difference in the incidence of anastomotic leakage among patients that did not have 


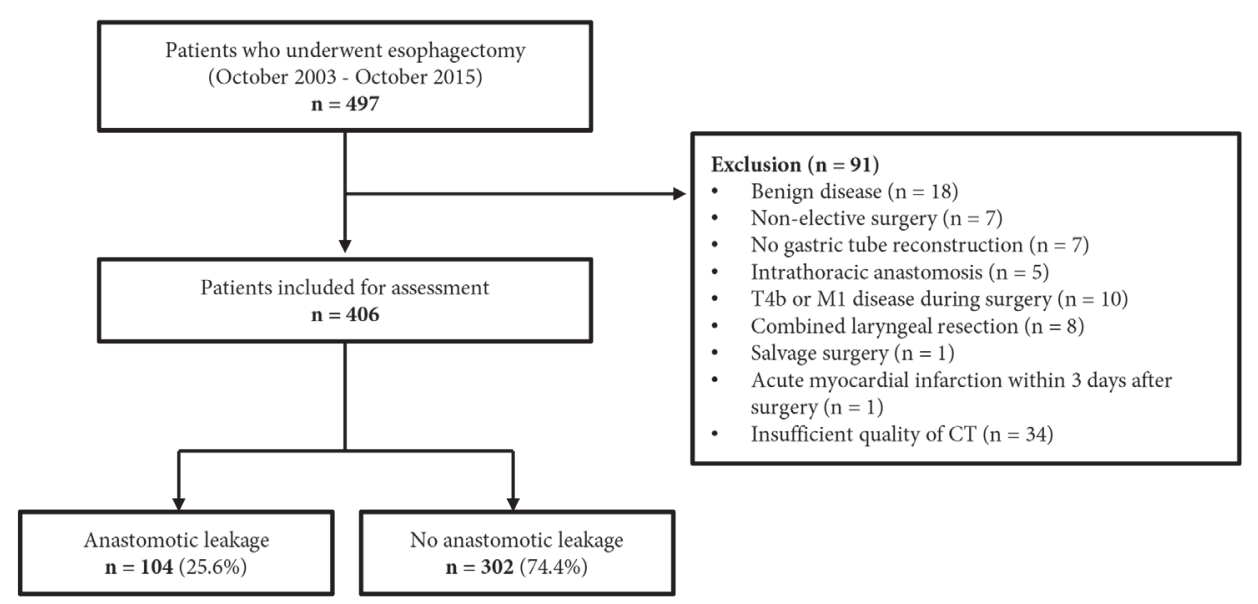

Figure 2. Flowchart of study design

calcifications at any location was 9\% (17\% (4/23) in patients without any calcifications versus $26 \%(100 / 383)$ in patients with calcifications).

Presence of calcification in the coronary arteries (minor calcification: $36.5 \%$ leakage [42/115], versus absence of calcification: $18.1 \%$ leakage [31/171], $p=.001$ ), supra-aortic arteries (minor calcification: 30.9\% leakage [25/81], major calcification: 35.3\% leakage [49/139], versus absence of calcification: 16.1\% leakage [30/186], $p=.007$ and $p<0.001$, respectively), and thoracic aorta (major calcification: 33.3\% leakage [40/120], versus absence of calcification: $19.4 \%$ leakage [28/144], $p=.011$ ), was significantly associated with an increased risk of anastomotic leakage in univariable logistic regression analysis. Although the prevalence of anastomotic leakage in patients with calcifications of the aortic valve, celiac axis, abdominal aorta, common and external iliac arteries appeared higher than in patients without calcification, these odds ratios were not statistically significant.

The calcification scores of the coronary arteries, supra-aortic arteries, thoracic aorta, abdominal aorta, celiac axis and external iliac arteries were entered per location into a multivariable logistic regression model, along with age, BMI, history of cardiovascular disease, COPD, diabetes mellitus and smoking (Table 3). Calcification of supra-aortic arteries remained significantly and independently associated with anastomotic leakage (OR 2.48, 95\% CI: 1.30-4.74, $p=.006$ for minor calcification and OR 2.72, 95\% CI: $1.49-4.99$, $p=.001$ for major calcification), as well as minor calcification of the coronary arteries (OR 2.29, 95\% CI: 1.28-4.12, $p=.005)$. As an illustration: the presence of minor calcification of supra-aortic arteries increased the odds of developing anastomotic leakage with $148 \%$ compared to patients without calcification of the supra-aortic arteries. 
Table 2. Baseline patient and treatment-related characteristics and their association with anastomotic leakage in univariable analysis.

\begin{tabular}{|c|c|c|c|}
\hline \multirow[t]{2}{*}{ Characteristic } & Anastomotic leakage & No anastomotic leakage & \multirow[t]{2}{*}{ p-value } \\
\hline & $\mathrm{n}=104(25.6 \%)$ & $\mathrm{n}=302(74.4 \%)$ & \\
\hline \multicolumn{4}{|l|}{ Patient-related } \\
\hline Age at diagnosis (years) & $65.5 \pm 8.8$ & $63.7 \pm 9.1$ & .082 \\
\hline Sex (male) & $82(78.8)$ & $218(72.2)$ & .182 \\
\hline BMI $\left(\mathrm{kg} / \mathrm{m}^{2}\right)$ & $25.8 \pm 4.3$ & $25.5 \pm 4.3$ & .538 \\
\hline ASA score & & & .156 \\
\hline 1 & $23(22.1)$ & $66(21.9)$ & \\
\hline 2 & $55(52.9)$ & $193(63.9)$ & \\
\hline 3 & $26(25.0)$ & $42(13.9)$ & \\
\hline 4 & $0(0.0)$ & $1(0.3)$ & \\
\hline \multicolumn{4}{|l|}{ Comorbidities } \\
\hline COPD & $24(23.1)$ & $37(12.3)$ & $.008^{*}$ \\
\hline Diabetes mellitus & $20(19.2)$ & $37(12.3)$ & .077 \\
\hline Cardiovascular $^{\mathrm{a}}$ & $46(44.2)$ & $110(36.4)$ & .158 \\
\hline Smoking status & & & 653 \\
\hline Current & $22(21.2)$ & $73(24.2)$ & \\
\hline Former & $44(42.3)$ & $113(37.4)$ & \\
\hline Never & $38(36.5)$ & $116(38.4)$ & \\
\hline Tumor histology & & & .742 \\
\hline Adenocarcinoma & $82(78.8)$ & $227(75.2)$ & \\
\hline Squamous cell carcinoma & $21(20.2)$ & $71(23.5)$ & \\
\hline Other $^{\mathrm{b}}$ & $1(1.0)$ & $4(1.3)$ & \\
\hline \multicolumn{4}{|l|}{ Treatment-related } \\
\hline Neoadjuvant treatment & & & .368 \\
\hline None & $38(36.5)$ & $93(30.8)$ & \\
\hline Chemotherapy & $26(25.0)$ & $96(31.8)$ & \\
\hline Chemoradiotherapy & $40(38.5)$ & $113(37.4)$ & \\
\hline Surgical approach & & & $.024^{*}$ \\
\hline Thoracolaparoscopic & $59(56.7)$ & $186(61.6)$ & \\
\hline Laparoscopic transhiatal & $24(23.1)$ & $42(13.9)$ & \\
\hline Thoracolaparotomic & $6(5.8)$ & $42(13.9)$ & \\
\hline Open transhiatal & $12(11.5)$ & $20(6.6)$ & \\
\hline Thoracoscopic-laparotomic & $3(2.9)$ & $12(4.0)$ & \\
\hline Anastomotic configuration & & & 649 \\
\hline End-to-side & $102(98.1)$ & $298(98.7)$ & \\
\hline End-to-end & $2(1.9)$ & $4(1.3)$ & \\
\hline Duration of surgery (minutes) & $343 \pm 115$ & $361 \pm 100$ & .121 \\
\hline Year of surgery & & & .280 \\
\hline $2003-2008$ & $25(24.0)$ & $56(18.5)$ & \\
\hline $2009-2010$ & $15(14.4)$ & $71(23.5)$ & \\
\hline 2011-2012 & $26(25.0)$ & $71(23.5)$ & \\
\hline 2013-2014 & $28(26.9)$ & $69(22.8)$ & \\
\hline 2015 & $10(9.6)$ & $35(11.6)$ & \\
\hline
\end{tabular}

Values are numbers of patients, with column-based percentages in parentheses or mean \pm SD.

* Significant difference based on $\chi^{2}$ test.

a Cardiovascular comorbidities defined as cardiac comorbidities (e.g. coronary artery bypass graft or valve replacement), vascular comorbidities (e.g. peripheral arterial disease), and/or hypertension.

b Adenosquamous carcinoma $(n=2)$, mixed adenoneuroendocrine carcinoma $(n=2)$ and carcinosarcoma $(n=1)$ 


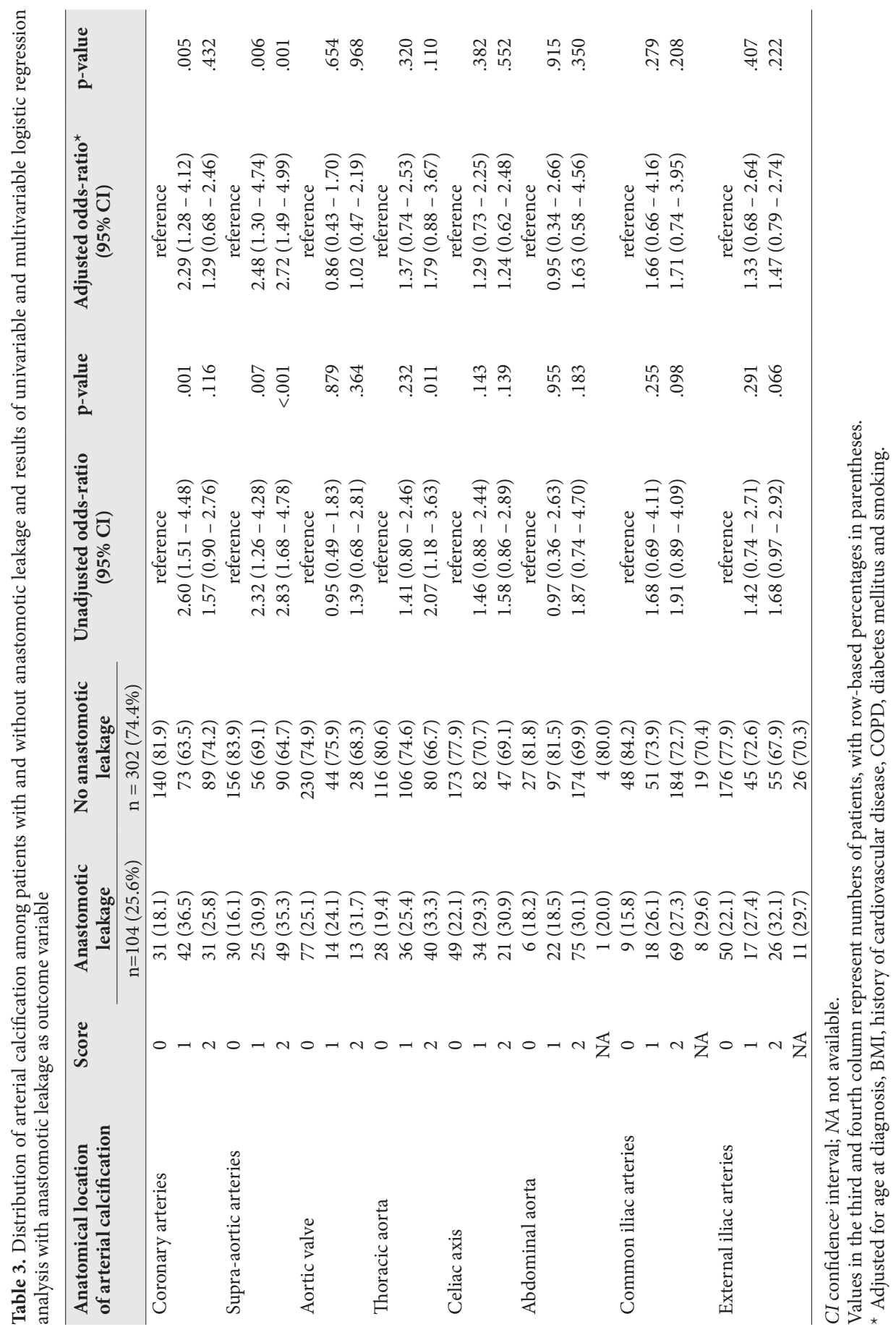




\section{Reproducibility of the calcification scoring system}

Reproducibility between and within observers was assessed in 30 randomly selected patients that appeared to be representative of the whole study group. The randomly selected patients had a mean age of 62 years \pm 11 (standard deviation) and $86 \%(26 / 30)$ were male. Anastomotic leakage occurred in 16.6\% (5/30).

The proportion of agreement for the calcification scores was high, ranging from $73.3 \%$ to $96.7 \%$ within one observer, and from $86.7 \%$ to $96.7 \%$ between observers for the different anatomical locations (Table 4).

Furthermore, the calcification score categories showed excellent intra- and interobserver reliability $(\kappa)$ for all anatomical locations, ranging from 0.63 to 0.96 and from 0.85 to 0.94 , respectively (Table 4$)$.

Table 4. Intra- and interobserver reliability and agreement of the arterial calcification scoring system per anatomical location

\begin{tabular}{|c|c|c|c|c|}
\hline \multirow{2}{*}{$\begin{array}{l}\text { Anatomical location of } \\
\text { arterial calcification }\end{array}$} & \multicolumn{2}{|c|}{ Intraobserver } & \multicolumn{2}{|c|}{ Interobserver } \\
\hline & $\begin{array}{l}\text { Reliability (к) } \\
(95 \% \text { CI })\end{array}$ & $\begin{array}{l}\text { Agreement }^{\mathrm{b}}(\%) \\
(95 \% \mathrm{CI})\end{array}$ & $\begin{array}{l}\text { Reliability }(\kappa)^{a} \\
(95 \% \text { CI })\end{array}$ & $\begin{array}{l}\text { Agreement }^{\mathrm{b}}(\%) \\
(95 \% \mathrm{CI})\end{array}$ \\
\hline Coronary arteries & $0.63(0.40-0.86)$ & $73.3(53.8-87.0)$ & $0.86(0.71-1.00)$ & $90.0(72.3-97.4)$ \\
\hline Supra-aortic arteries & $0.87(0.74-0.99)$ & $86.7(68.4-95.6)$ & $0.90(0.79-1.00)$ & $90.0(72.3-97.4)$ \\
\hline Aortic valve & $0.87(0.71-1.00)$ & $93.3(76.5-98.8)$ & $0.94(0.83-1.00)$ & $96.7(81.0-99.8)$ \\
\hline Thoracic aorta & $0.86(0.73-0.99)$ & $86.7(68.4-95.6)$ & $0.86(0.73-0.99)$ & 86.7 (68.4-95.6) \\
\hline Celiac axis & $0.81(0.62-0.99)$ & $86.7(68.4-95.6)$ & $0.90(0.72-1.00)$ & 96.7 (81.0-99.8) \\
\hline Abdominal aorta & $0.91(0.79-1.00)$ & $93.3(76.5-98.8)$ & $0.91(0.78-1.00)$ & $93.3(76.5-98.8)$ \\
\hline Common iliac arteries & $0.96(0.88-1.00)$ & $96.7(81.0-99.8)$ & $0.88(0.74-1.00)$ & $90.0(72.3-97.4)$ \\
\hline External iliac arteries & $0.96(0.89-1.00)$ & $96.7(81.0-99.8)$ & $0.85(0.71-0.99)$ & $86.7(68.4-95.6)$ \\
\hline
\end{tabular}

a Reliability was assessed by using Cohen's linearly weighted kappa $(\kappa)$. The weighted $\kappa$ statistic can be interpreted as follows: $\kappa$ of 0.81 to 1.00 indicates excellent reliability; $\kappa$ of 0.61 to 0.80 , good reliability; $\kappa$ of 0.41 to 0.60 , moderate reliability; $\kappa$ of 0.21 to 0.40 , fair reliability; and $\kappa$ of less than 0.20 , poor reliability. ${ }^{23}$

b Proportion of agreement between calcification scores. 


\section{Discussion}

Anastomotic leakage after esophagectomy remains one of the most devastating complications after esophagectomy, and has an adverse impact on prognosis. ${ }^{1-6,9}$ Many patient and surgery-related risk factors for anastomotic leakage have been reported in literature, but fail to accurately predict the risk of anastomotic leakage. The results of this cohort study of 406 patients did not show a significant association between anastomotic leakage and characteristics such as gender, age, BMI, ASA score, cardiovascular comorbidity, diabetes mellitus and smoking. A history of COPD was the only patient-related factor significantly associated with anastomotic leakage in univariable analysis in this cohort. The chronic use of steroids by these patients has been suggested as a possible explanation for impaired anastomotic healing. ${ }^{2,15}$ In multivariable analysis however, no standard patientrelated characteristics remained significantly associated with anastomotic leakage. This highlights the multifactorial nature of anastomotic leakage and the challenging prediction of this complication.

In this study, the presence of calcification in the coronary and supra-aortic arteries was proven to be independently associated with anastomotic leakage, irrespective of clinical and treatment-related characteristics. These calcifications may be a predisposing factor or consequence of diffuse arterial disease, and could therefore be helpful to identify patients at risk for anastomotic leakage. ${ }^{11}$ Recognition of the increased risk for developing anastomotic leakage without requiring additional imaging could aid the complex decisionmaking process of surgical treatment of patients with esophageal cancer. High risk patients (e.g. patients with supra-aortic calcification score $\geq 1$ have a 33.6\% (74/220) risk of anastomotic leakage) could be more fully optimized before surgery by means of preventive measures to reduce their risk of anastomotic leakage. Important preoperative interventions to enhance postoperative outcome may include physical therapy to increase cardiorespiratory function and improvement of nutritional status. ${ }^{24-26}$

To improve preoperative identification of patients at high risk for anastomotic leakage, the current study performed extensive measurements of cardiovascular features on routine preoperative CT images to explore their association with anastomotic leakage. The findings of the current study demonstrate that, not only locoregional vascular disease as demonstrated in previous studies, ${ }^{11,15}$ but also generalized vascular disease is indicative for the risk of anastomotic leakage after esophagectomy. This result adds to the current evidence of the association between atherosclerotic calcification and leakage of gastrointestinal anastomoses, which is presumably based on ischemia of the anastomosis. ${ }^{11,15,27-30}$

In line with the ischemia hypothesis as contributing factor to the development of anastomotic leakage, it has been suggested that patients at high risk of anastomotic leakage based on their vascular status could be included in ischemic conditioning trials. ${ }^{10,11}$ Ischemic 
conditioning increases perfusion of the gastric tube by partial gastric devascularization through arterial embolization or laparoscopic arterial ligation, followed by esophagectomy and anastomosis at a second stage. ${ }^{10}$ However, currently there is insufficient evidence available to support widespread implementation of ischemic conditioning of the stomach in order to decrease anastomotic leakage rates in clinical practice. ${ }^{10,31}$ Specifically selecting patients that are high at risk of developing anastomotic leakage (i.e. patients with minor or major arterial calcification) may improve the effect of this technique on anastomotic leakage rates. ${ }^{10,11}$

Various limitations apply to this study. First, no internal validation of our results was performed, and external validation of our results is warranted to test the generalizability of these results in other populations (e.g. with a different prevalence of leakage, use of other surgical techniques). Second, there are some potential limitations of the visual grading system. To facilitate an easy and fast visual calcification grading system, calcification was stratified between minor and major calcification based on the number of calcified foci within a trajectory in this study. As a result, the exact number of calcified foci and their extent were not separately analyzed and thus the calcification load could vary largely within one score. Moreover, the cut off points for the number of calcifications within one score are not adjusted for a patient's individual body height and varying anatomy of the arteries. Therefore, this simplified system could have over- or underestimated the calcification load within a patient.

Despite these potential limitations, several advantages apply to this approach. The grading system used in this study is easy to use and allows for a fast differentiation of calcification burden. This is confirmed by the high reproducibility of the calcification grading system, shown by the excellent intra- and interobserver agreement of the current and previous related studies. ${ }^{12,15,16}$ Furthermore, for the prediction of cardiovascular events this grading system has proven to be equally efficient as more challenging scoring systems that are based on plaques or irregularities of the vascular wall. ${ }^{12}$ Second, the scoring system does not require any special CT protocol or calcium-scoring software. Last, a CT or a combined positron emission tomography (PET)/CT scan is the principle staging tool for esophageal cancer. Obtaining information on arterial calcification therefore does not require any additional tests or imaging.

In conclusion, this study demonstrates that calcification of the coronary and supra-aortic arteries is an independent predictor of anastomotic leakage following esophagectomy. These results demonstrate that generalized vascular disease is more indicative for the risk of leakage than local vascular disease. This new described risk factor for anastomotic leakage after esophagectomy may be used in future prediction models, and eventually aid to a more individualized identification of the risk of anastomotic leakage following esophagectomy. 


\section{References}

1. van Hagen P, Hulshof MCCM, van Lanschot JJB, et al. Preoperative Chemoradiotherapy for Esophageal or Junctional Cancer. N Engl J Med. 2012;366(22):2074-84.

2. Kassis ES, Kosinski AS, Ross P, Koppes KE, Donahue JM, Daniel VC. Predictors of anastomotic leak after esophagectomy: An analysis of the society of thoracic surgeons general thoracic database. Ann Thorac Surg. 2013;96(6):1919-26.

3. Biere SSAY, Maas KW, Cuesta MA, van der Peet DL. Cervical or Thoracic Anastomosis after Esophagectomy for Cancer: A Systematic Review and Meta-Analysis. Dig Surg. 2011;28(1):29-35.

4. Urschel J. Esophagogastrostomy anastomotic leaks complicating esophagectomy: A review. Am J Surg. 1995;169(6):634-40.

5. Raymond DP, Seder CW, Wright CD, et al. Predictors of Major Morbidity or Mortality After Resection for Esophageal Cancer: A Society of Thoracic Surgeons General Thoracic Surgery Database Risk Adjustment Model. Ann Thorac Surg. 2016;102(1):207-14.

6. Kataoka K, Takeuchi H, Mizusawa J, et al. Prognostic Impact of Postoperative Morbidity After Esophagectomy for Esophageal Cancer: Exploratory Analysis of JCOG9907. Ann Surg. 2017;265(6):1152-7.

7. Maas K, Cuesta M, van Berge Henegouwen M, et al. Quality of Life and Late Complications After Minimally Invasive Compared to Open Esophagectomy: Results of a Randomized Trial. World J Surg. 2015;39(8):198693.

8. Schuchert M, Abbas G, Nason K, et al. Impact of anastomotic leak on outcomes after transhiatal esophagectomy. Surgery. 2010;148(4):831-40.

9. Markar S, Gronnier C, Duhamel A, et al. The Impact of Severe Anastomotic Leak on Long-term Survival and Cancer Recurrence After Surgical Resection for Esophageal Malignancy. Ann Surg. 2015;262(6):97280.

10. Kechagias A, van Rossum PSN, Ruurda JP, van Hillegersberg R. Ischemic Conditioning of the Stomach in the Prevention of Esophagogastric Anastomotic Leakage After Esophagectomy. Ann Thorac Surg. 2016;101(4):1614-23.

11. Goense L, van Rossum PSN, Weijs TJ, et al. Aortic Calcification Increases the Risk of Anastomotic Leakage After Ivor-Lewis Esophagectomy. Ann Thorac Surg. 2016;102(1):247-52.

12. Gondrie M, Mali W, Jacobs P, Oen A, van der Graaf Y, PROVIDI study group. Cardiovascular disease: prediction with ancillary aortic findings on chest CT scans in routine practice. Radiology. 2010;257(2):54959.

13. de Jong P, Gondrie M, Buckens C, Jacobs P, Mali W, van der Graaf Y. Prediction of cardiovascular events by using non-vascular findings on routine chest CT. PLoS One. 2011;6(10):e26036.

14. Jairam P, Gondrie M, Grobbee D, Mali W, Jacobs P, van der Graaf Y. Unrequested imaging findings from routine chest CT identify subjects at high risk of future cardiovascular events. Radiology. 2014;272(3):7008.

15. van Rossum P, Haverkamp L, Verkooijen HM, van Leeuwen M, van Hillergersberg R, Ruurda J. Calcification of Arteries Supplying the Gastric Tube: A New Risk Factor for Anastomotic Leakage after Esophageal Surgery. Radiology. 2015;274(1):124-32.

16. Jacobs PCA, Prokop M, Oen AL, van der Graaf Y, Grobbee DE, Mali WPTM. Semiquantitative assessment of cardiovascular disease markers in multislice computed tomography of the chest: interobserver and intraobserver agreements. J Comput Assist Tomogr. 2010;34(2):279-84.

17. Sun G-W, Shook TL, Kay GL. Inappropriate use of bivariable analysis to screen risk factors for use in multivariable analysis. J Clin Epidemiol. 1996;49(8):907-16.

18. Moons KGM, Altman DG, Reitsma JB, et al. Transparent Reporting of a multivariable prediction model for Individual Prognosis Or Diagnosis (TRIPOD): Explanation and Elaboration. Ann Intern Med. 2015;162(1):W1.

19. Moons KGM, Royston P, Vergouwe Y, Grobbee DE, Altman DG. Prognosis and prognostic research: what, why, and how? BMJ. 2009;338(feb23 1):b375.

20. Moons K, Donders R, Stijnen T, Harrell FJ. Using the outcome for imputation of missing predictor values was preferred. J Clin Epidemiol. 2006;59(10):1092-110.

21. Vergouwe Y, Royston P, Moons K, Altman D. Development and validation of a prediction model with missing predictor data: a practical approach. J Clin Epidemiol. 2010;63(2):205-14. 
22. Cohen J. Weighted kappa: nominal scale agreement with provision for scaled disagreement or partial credit. Psychological Bulletin. 1968;70(4):213-20.

23. Landis J, Koch G. The measurement of observer agreement for categorical data. Biometrics. 1977;33(1):15974 .

24. Inoue J, Ono R, Makiura D, et al. Prevention of postoperative pulmonary complications through intensive preoperative respiratory rehabilitation in patients with esophageal cancer. Dis Esophagus. 2013;26(1):68-74.

25. Bower MR, Martin RCG. Nutritional management during neoadjuvant therapy for esophageal cancer. $J$ Surg Oncol. 2009;100(1):82-7.

26. van Adrichem EJ, Meulenbroek RL, Plukker JTM, Groen H, van Weert E. Comparison of two preoperative inspiratory muscle training programs to prevent pulmonary complications in patients undergoing esophagectomy: a randomized controlled pilot study. Ann Surg Oncol. 2014;21(7):2353-60.

27. Komen N, Klitsie P, Dijk JW, et al. Calcium score: a new risk factor for colorectal anastomotic leakage. Am J Surg. 2011;201(6):759-65.

28. Pierie J, De GraafP, Poen H, Van der Tweel I, Obertop H. Impaired healing of cervical oesophagogastrostomies can be predicted by estimation of gastric serosal blood perfusion by laser Doppler flowmetry. Eur J Surg. 1994;160(11):599-603.

29. Ikeda Y, Niimi M, Kan S, Shatari T, Takami H, Kodaira S. Clinical significance of tissue blood flow during esophagectomy by laser Doppler flowmetry. J Thorac Cardiovasc Surg. 2001;122:1101-6.

30. Zehetner J, DeMeester S, Alicuben E, et al. Intraoperative assessment of perfusion of the gastric graft and correlation with anastomotic leaks after esophagectomy. Ann Surg. 2015;262(1):74-8.

31. Markar SR, Arya S, Karthikesalingam A, Hanna GB. Technical Factors that Affect Anastomotic Integrity Following Esophagectomy: Systematic Review and Meta-analysis. Ann Surg Oncol. 2013;20(13):4274-81. 


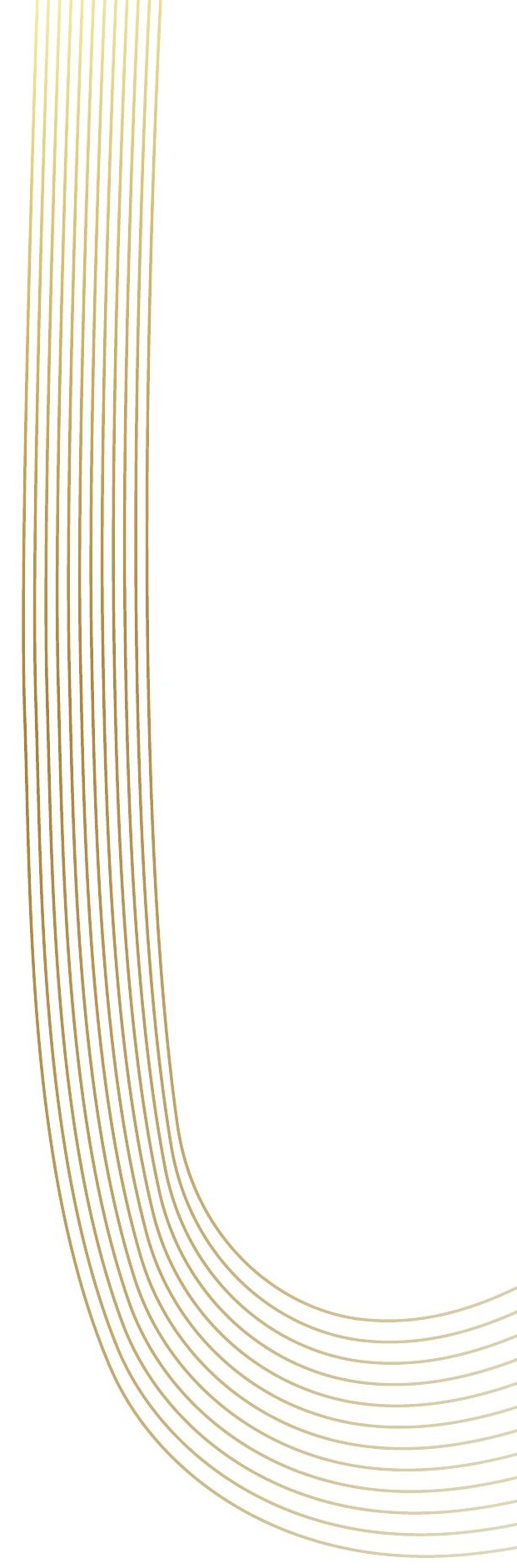


Safety and feasibility of minimally invasive surgical interventions for esophageal and gastric cancer in the acute setting: a nationwide cohort study

Submitted

A.S. Borggreve, B.F. Kingma, J.P. Ruurda, R. van Hillegersberg, Dutch Upper GI Cancer Audit (DUCA) group 


\begin{abstract}
Background

Minimally invasive esophagectomy and gastrectomy are increasingly performed and might be superior to their open equivalents in an elective setting. The aim of this study was to evaluate whether minimally invasive approaches can be safely applied in the acute setting as well.
\end{abstract}

\title{
Methods
}

All patients who underwent an acute surgical intervention for primary esophageal or gastric cancer between 2011-2017 were identified from the nationwide database of the Dutch Upper GI Cancer Audit (DUCA). Conversion rates, postoperative complications, reinterventions, postoperative mortality, hospital stay and oncological outcomes (radical resection rates and median lymph node yield) were evaluated.

\section{Results}

Between 2011-2017, surgery for esophagogastric cancer was performed in an acute setting in $2 \%(190 / 8861)$ in The Netherlands. A total of 14 acute resections for esophageal cancer were performed, which included 7 minimally invasive esophagectomies and 7 open esophagectomies. As these numbers were very low, no comparison between minimally invasive and open esophagectomies was made.

A total of 122 acute resections for gastric cancer were performed, which included 39 minimally invasive gastrectomies and 83 open gastrectomies. Conversion occurred in 9 patients (23\%). Minimally invasive gastrectomy was at least comparable to open gastrectomy regarding postoperative complications (36\% versus 51\%), median hospital stay (9 days [IQR: 7-16 days] versus 11 days [IQR: 7-17 days]), readmissions (8\% versus 11\%) and oncological outcomes (radical resection rate: $87 \%$ versus $66 \%$, median lymph node yield: 21 [IQR: 15-32] versus 16 [IQR: 11-24]).

\section{Conclusions}

Minimally invasive surgery for gastric cancer is safe and feasible in the acute setting, with at least comparable postoperative clinical and short-term oncological outcomes compared to open surgery but a relatively high conversion rate. 


\section{Introduction}

Minimally invasive surgical techniques are increasingly being applied in the surgical treatment of esophageal and gastric cancer. ${ }^{1,2}$ Evidence from randomized controlled trials and nationwide studies suggests that these techniques might provide benefits over the traditional open approaches, especially regarding short-term outcomes in terms of postoperative morbidity and length of hospital stay. ${ }^{3-12}$ In contrast, higher reintervention rates were observed after minimally invasive esophagectomies in population-based studies. ${ }^{5-8}$ As the aforementioned studies only included patients who underwent an elective surgical resection, the generalizability of these results to the acute setting might be limited. Acute surgery for esophageal and gastric cancer is relatively rare and is usually only performed in case tumors are actively bleeding or have perforated. These cases are different from the usual elective patient population and might therefore be more difficult to treat with minimally invasive surgical techniques. More research is warranted to investigate the role of minimally invasive surgical techniques for patients with esophageal and gastric cancer who have an acute indication for a resection. Therefore, the aim of this nationwide cohort study was to describe the postoperative outcomes of minimally invasive as compared to open acute surgery for esophageal and gastric cancer.

\section{Methods}

\section{Study design}

This nationwide observational cohort study was conducted with data from the Dutch Upper GI Cancer Audit (DUCA), a nationwide registration of all patients undergoing surgery for esophageal and gastric cancer since $2011 .{ }^{13}$ Registration of cases in the DUCA is mandatory for each hospital and includes patient characteristics, treatment details including the timing of surgery, postoperative outcomes (until 30 days after surgery), and pathological outcomes. The scientific committee of the DICA and DUCA approved this study. No ethical approval or informed consent was required under Dutch law.

\section{Study population and treatment}

All patients who underwent acute surgery with the intention to perform an esophagectomy or gastrectomy for cancer between 2011-2017 were selected from the DUCA registry. Patients were diagnosed according to the Dutch national guidelines for diagnosis, treatment, follow up, and guidance of patients for patients with esophageal and gastric cancer. ${ }^{14,15}$ Acute surgical interventions were either defined as emergent (i.e. surgery scheduled $<12$ hours after presentation with an acute indication) or urgent (surgery scheduled $>12$ hours 
- but not electively - after presentation with an acute indication). Both were included in the current study. Exclusion criteria were prophylactic surgical indications (i.e. no proven malignancy), insufficient data regarding the tumor or surgical approach, and surgery for cancer recurrence.

\section{Outcome measures}

The outcome measures included the rates of conversion to an open procedure, postoperative complications, reinterventions, postoperative mortality (i.e. mortality during initial hospital admission or within 30 days after surgery), length of postoperative stay on the Intensive Care Unit (ICU) and in hospital, as well as readmissions ( $<30$ days after discharge). Complications were defined according to standards of the DUCA, and included pulmonary complications (clinically proven pneumonia, pleural effusion leading to drainage, pleural empyema and/or acute respiratory distress syndrome), anastomotic leakage (either by clinical or radiological diagnosis), cardiac complications (supraventricular and ventricular arrhythmia, myocardial infarction and/or heart failure), thromboembolic complications (pulmonary embolism, deep venous thrombosis, stroke and/or thrombophlebitis), neurologic complications (recurrent laryngeal nerve injury and/or acute delirium), urologic complications (urinary tract infection, urinary retention and/or renal insufficiency), intraabdominal abscess, chyle leakage, fascia dehiscence and wound infections. Furthermore, oncological outcomes in terms of radicality and total lymph node yield were analyzed. A radical resection (i.e. R0) was defined as the absence of tumor cells within the resection margins of the resection specimen.

\section{Statistical analyses}

Patient and treatment characteristics were described as counts with percentages, mean ( \pm standard deviation $[S D]$ ) or median (interquartile range [IQR]). The postoperative outcomes were separately described for patients who underwent minimally invasive surgery and open group. No tests for statistical significance of differences between groups were performed because of inability to adequately correct for confounding bias due to small group sizes. Statistical analyses were performed using SPSS 23.0 (IBM Corp., Armonk, NY, USA).

\section{Results}

\section{Study population}

Between 2011-2017, a total of 8861 patients who underwent surgery for esophageal or gastric cancer in the Netherlands were registered in the DUCA. Surgery was performed in 


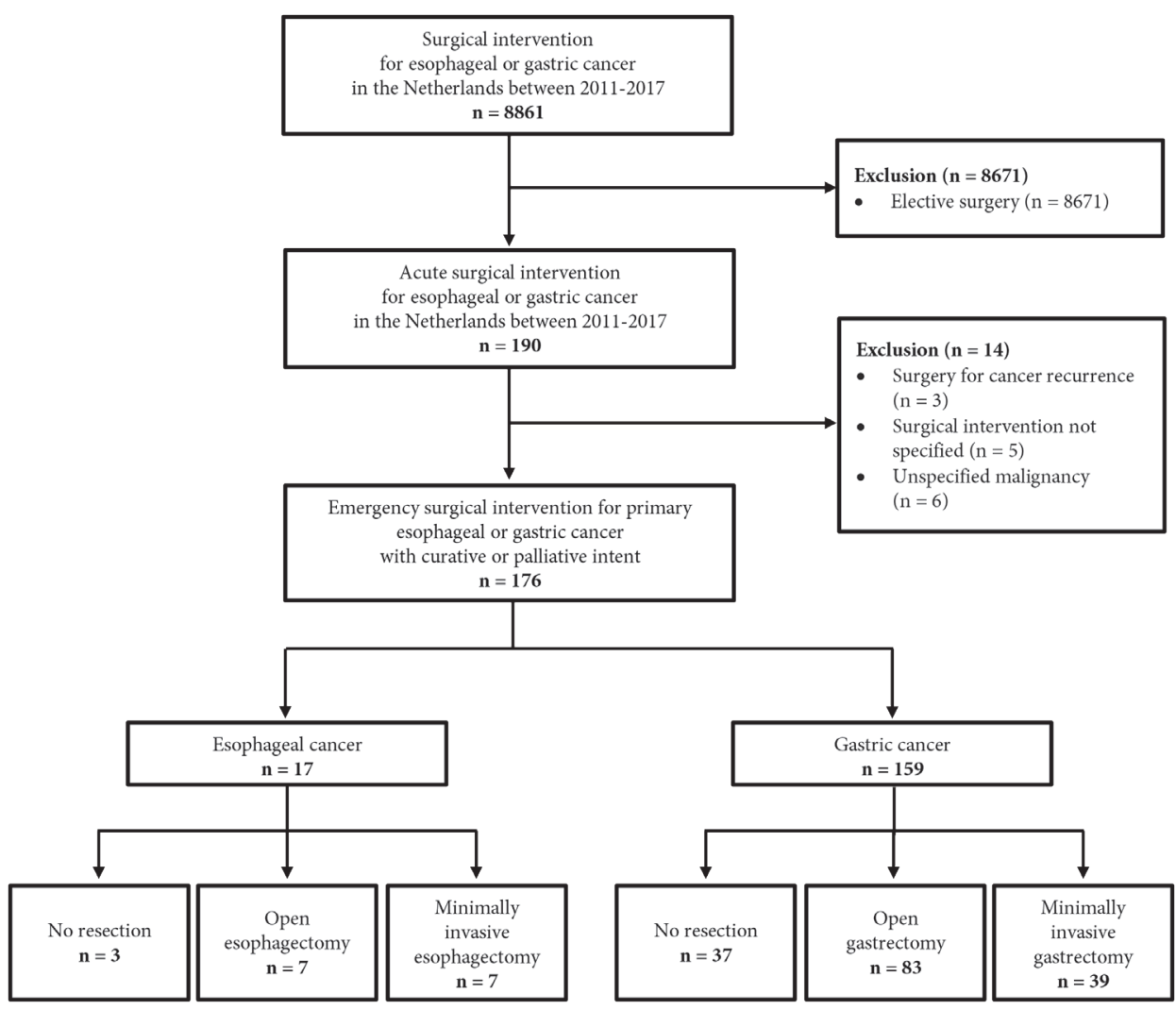

Figure 1. Study flowchart

an acute setting in 190 out of these 8861 patients (2\%). Patients who underwent an unspecified surgical intervention $(n=5)$, a surgical procedure for cancer recurrence $(n=3)$, or a surgical procedure for an unspecified malignancy $(n=6)$ were excluded. Hence, a total of 176 patients were included, of whom 17 patients with esophageal cancer and 159 patients with gastric cancer (Figure 1).

\section{Acute surgical interventions for esophageal cancer}

Acute surgical interventions for esophageal cancer were performed in 17 patients and were with curative intent in all of these patients. The median number of days between the diagnostic biopsy and surgery was 70 days (IQR 46 - 121 days). In 2 patients (12\%), no histologic diagnosis was present prior to surgery. The reason for surgery was a perforation in 7 patients (41\%), bleeding in 5 patients (29\%) and other (not specified) in the remaining 5 cases $(29 \%)$. 
Table 1. Baseline characteristics of patients who underwent an acute surgical intervention for esophageal cancer in the Netherlands between 2011 and 2017.

\begin{tabular}{|c|c|c|}
\hline \multirow[t]{2}{*}{ Characteristics } & \multicolumn{2}{|c|}{ All } \\
\hline & $\mathrm{n}=17$ & $\%$ \\
\hline \multicolumn{3}{|l|}{ Patiënt-related characteristics } \\
\hline Age, years $($ mean $\pm S D)$ & \multicolumn{2}{|c|}{$64 \pm 11$} \\
\hline \multicolumn{3}{|l|}{ Sex } \\
\hline Male & 15 & $88 \%$ \\
\hline Female & 2 & $12 \%$ \\
\hline BMI, $\mathbf{k g} / \mathbf{m}^{2}($ mean $\pm \mathrm{SD})$ & \multicolumn{2}{|c|}{$26 \pm 5$} \\
\hline \multicolumn{3}{|l|}{ ASA classification } \\
\hline I & 2 & $12 \%$ \\
\hline II & 7 & $41 \%$ \\
\hline III & 7 & $41 \%$ \\
\hline IV & 1 & $6 \%$ \\
\hline \multicolumn{3}{|l|}{ Comorbidities } \\
\hline Cardiac & 2 & $12 \%$ \\
\hline Vascular & 8 & $47 \%$ \\
\hline Diabetes & 5 & $29 \%$ \\
\hline Pulmonary & 3 & $18 \%$ \\
\hline Previous abdominal or thoracic surgery & 7 & $41 \%$ \\
\hline \multicolumn{3}{|l|}{ Tumor location } \\
\hline Middle esophagus & 1 & $6 \%$ \\
\hline Distal esophagus & 9 & $53 \%$ \\
\hline Gastro-esophageal junction & 5 & $29 \%$ \\
\hline Unknown & 2 & $12 \%$ \\
\hline \multicolumn{3}{|l|}{ Histology } \\
\hline Adenocarcinoma & 15 & $88 \%$ \\
\hline Squamous cell carcinoma & 1 & $6 \%$ \\
\hline Other & 1 & $6 \%$ \\
\hline \multicolumn{3}{|l|}{ cT status ${ }^{\star}$} \\
\hline $\mathrm{T} 1$ & 3 & $18 \%$ \\
\hline $\mathrm{T} 2$ & 2 & $12 \%$ \\
\hline T3 & 5 & $29 \%$ \\
\hline $\mathrm{T} 4$ & 5 & $29 \%$ \\
\hline $\mathrm{Tx}$ & 2 & $12 \%$ \\
\hline \multicolumn{3}{|l|}{ cN status* } \\
\hline No & 6 & $35 \%$ \\
\hline $\mathrm{N}+$ & 8 & $47 \%$ \\
\hline $\mathrm{Nx}$ & 3 & $18 \%$ \\
\hline \multicolumn{3}{|l|}{ cM status* } \\
\hline M0 & 13 & $76 \%$ \\
\hline M1 & 2 & $12 \%$ \\
\hline $\mathrm{Mx}$ & 2 & $12 \%$ \\
\hline
\end{tabular}


Table 1. Continued

\begin{tabular}{|c|c|c|}
\hline \multirow[t]{2}{*}{ Characteristics } & \multicolumn{2}{|c|}{ All } \\
\hline & $\mathbf{n}=17$ & $\%$ \\
\hline \multicolumn{3}{|l|}{ Treatment-related characteristics } \\
\hline \multicolumn{3}{|l|}{ Neoadjuvant therapy ${ }^{\dagger}$} \\
\hline None & 10 & $59 \%$ \\
\hline Chemoradiotherapy & 4 & $24 \%$ \\
\hline Chemotherapy & 2 & $12 \%$ \\
\hline Radiotherapy & 1 & $6 \%$ \\
\hline \multicolumn{3}{|l|}{ Setting } \\
\hline Emergent ( $<12$ hours) & 10 & $59 \%$ \\
\hline Urgent ( $>12$ hours) & 8 & $41 \%$ \\
\hline \multicolumn{3}{|l|}{ Reason for emergency surgery } \\
\hline Bleeding & 5 & $29 \%$ \\
\hline Perforation & 7 & $41 \%$ \\
\hline Other/unknown & 5 & $29 \%$ \\
\hline \multicolumn{3}{|l|}{ Surgical approach } \\
\hline Minimally invasive & 9 & $53 \%$ \\
\hline Open & 8 & $47 \%$ \\
\hline \multicolumn{3}{|l|}{ Surgical procedure } \\
\hline Transhiatal esophagectomy & 9 & $53 \%$ \\
\hline Transthoracic esophagectomy & 5 & $29 \%$ \\
\hline Diagnostic thoracotomy & 1 & $6 \%$ \\
\hline Diagnostic laparoscopy & 2 & $12 \%$ \\
\hline \multicolumn{3}{|l|}{ Reconstruction } \\
\hline Gastric conduit reconstruction & 12 & $71 \%$ \\
\hline No reconstruction & 4 & $24 \%$ \\
\hline Unknown & 1 & $6 \%$ \\
\hline \multicolumn{3}{|l|}{ Location of anastomosis } \\
\hline Cervical & 10 & $59 \%$ \\
\hline Intrathoracic & 2 & $12 \%$ \\
\hline Not applicable & 5 & $29 \%$ \\
\hline Lymph node dissection & 13 & $76 \%$ \\
\hline \multicolumn{3}{|l|}{ Year of surgery } \\
\hline $2011-2013$ & 9 & $53 \%$ \\
\hline $2014-2017$ & 8 & $47 \%$ \\
\hline
\end{tabular}

ASA American Society of Anesthesiologists; BMI body mass index at diagnosis; SD standard deviation Note. Data are numbers of patients with column-based percentages in parentheses, unless otherwise stated.

* Clinical T status and N status are based on AJCC TNM 7th edition.

† The standard regimen for neoadjuvant treatment for esophageal cancer patients in the Netherlands consists of carboplatin and paclitaxel, weekly during 5 weeks, and concurrent radiotherapy with a total radiation dose of 41.4 Gy in 23 fractions of 1.8 Gy. For gastro-esophageal junction or gastric adenocarcinoma, peri-operative treatment generally consists of chemotherapy regimens similar to the MAGIC-trial (epirubicin, cisplatin and capecitabine). 
Table 2. Short-term outcomes for patients who underwent an acute esophagectomy for esophageal cancer in the Netherlands between 2011 and 2017.

\begin{tabular}{|c|c|c|}
\hline & \multicolumn{2}{|c|}{ Total } \\
\hline & $\mathrm{n}=14$ & $\%$ \\
\hline \multicolumn{3}{|l|}{ Peroperative outcomes } \\
\hline Conversion $^{*}$ & 1 & $7 \%$ \\
\hline \multicolumn{3}{|l|}{ Postoperative complications } \\
\hline All & 7 & $50 \%$ \\
\hline Pulmonary $\dagger$ & 6 & $43 \%$ \\
\hline Anastomotic leakage $\ddagger$ & 4 & $29 \%$ \\
\hline Cardiac $\$$ & 1 & $7 \%$ \\
\hline Chyle leakage & 0 & $0 \%$ \\
\hline Reinterventions & $1^{* *}$ & $7 \%$ \\
\hline \multicolumn{3}{|l|}{ Recovery } \\
\hline ICU duration (median, IQR) & 3 & $(1-6)$ \\
\hline Length of stay (median, IQR) & 13 & $(11-26)$ \\
\hline Postoperative mortality & 1 & $7 \%$ \\
\hline Readmission to hospital \# & 0 & $0 \%$ \\
\hline \multicolumn{3}{|l|}{ Pathological outcomes } \\
\hline \multicolumn{3}{|l|}{ Radicality } \\
\hline R0 & 9 & $64 \%$ \\
\hline $\mathrm{R} 1$ & 5 & $36 \%$ \\
\hline Lymph node yield (median, IQR) & 21 & $(12-26)$ \\
\hline Positive lymph nodes harvested (median, IQR) & 3 & $(1-10)$ \\
\hline
\end{tabular}

$I Q R$ interquartile range, $N A$ not applicable

Note. There were no missing values for the variables described in this table.

* Conversion $>30$ minutes after start of surgery because of peroperative bleeding.

+ Pneumonia, pleural effusion, respiratory failure, pneumothorax and/or acute respiratory distress syndrome.

* Any clinically or radiologically proven anastomotic leakage.

s Supraventricular and ventricular arrhythmia, myocardial infarction and/or heart failure.

s Death during initial hospital admission or within 30 days after surgery.

\# Readmission to hospital within 30 days after initial discharge.

${ }^{* *}$ Re-operation for anastomotic leakage.

Esophagectomy was performed in 14 out of these 17 patients (82\%); by a minimally invasive transhiatal approach in 4 patients, an open transhiatal approach in 5 patients, a minimally invasive transthoracic approach in 3 patients, and an open transthoracic approach in 2 patients. The remaining 3 patients (18\%) underwent a surgical procedure without resection, which involved a diagnostic laparoscopy in 2 patients and a diagnostic thoracotomy in 1 patient. A complete overview of patient and treatment characteristics of the esophageal cancer patients is presented in Table 1. 
Perioperative and oncological outcomes of the 14 patients who underwent esophagectomy are shown in Table 2 . The outcomes were not reported for minimally invasive esophagectomy and open esophagectomy separately, as the number of patients for both groups was only 7 . Postoperative complications occurred in 50\% (7/14). Pulmonary complications were most common $(43 \%, 6 / 14)$, followed by anastomotic leakage $(29 \%, 4 / 14)$. The median length of hospital stay was 13 days (IQR: 11-26 days). No patients were readmitted to the hospital within 30 days after discharge.

Histopathological evaluation of the resection specimen showed that a radical resection was achieved in 64\% (9/14). The median lymph node yield was 21 (IQR: 12-26).

\section{Acute surgical interventions for gastric cancer}

An acute surgical intervention for gastric cancer was performed in 159 patients, with an upfront curative intent in the majority of patients (70\%). The median time between diagnostic biopsy and acute surgery was 23 days (IQR 11 - 36 days). Histological confirmation of gastric cancer was not present prior to surgery in 25 patients (16\%). The reason for surgery was a bleeding in 68 patients (43\%), perforation in 19 patients (12\%) and other (not specified) in the remaining 73 patients (46\%). Gastrectomy was performed in 122 patients (77\%), which was by a minimally invasive approach in 39 patients and by an open approach in 83 patients. The remaining 37 patients (23\%) underwent a surgical procedure without resection, which involved a gastroenterostomy in 26 patients, diagnostic laparoscopy in 6 patients and a diagnostic laparotomy in 5 patients. Most minimally invasive procedures were observed in the more recent years (Figure 2). A complete overview of patient and treatment-related characteristics of the gastric cancer patients is presented in Table 3.

Perioperative and oncological outcomes of the 122 patients who underwent a gastrectomy are shown in Table 4. Conversion to an open procedure occurred in 9 of the 39 minimally invasive gastrectomies (23\%), mostly because of the extent of the tumor and poor exposure (Table 4). Postoperative complications occurred in 14 of the 39 patients who underwent a minimally invasive gastrectomy (36\%) and in 42 of the 83 patients who underwent an open gastrectomy $(51 \%)$. When comparing minimally invasive gastrectomy versus open gastrectomy, pulmonary complications occurred in $13 \%$ versus $18 \%$, anastomotic leakage in $8 \%$ versus $8 \%$, and wound infection in $0 \%$ versus $13 \%$, respectively. Re-interventions, mostly for anastomotic leakage in both groups, were performed in $23 \%$ after a minimally invasive gastrectomy versus $14 \%$ after an open gastrectomy. Postoperative mortality occurred in $8 \%$ after a minimally invasive gastrectomy versus $11 \%$ after an open gastrectomy. The median length of hospital stay was 9 days (IQR: 7-16 days) after minimally invasive gastrectomy versus 11 days (IQR: 7-17 days) after open gastrectomy. Readmission to the hospital was seen in $8 \%$ after minimally invasive gastrectomy versus $11 \%$ after open gastrectomy. 
Histopathological evaluation of the resection specimen showed that a radical resection was achieved in 34 of the minimally invasive gastrectomies (87\%) and in 55 of the open gastrectomies (66\%). The median lymph node yield was 21 (IQR: 15-32) after minimally invasive gastrectomy and 16 (IQR: 11-24) after open gastrectomy.

A

Surgical interventions for gastric cancer $(n=159)$

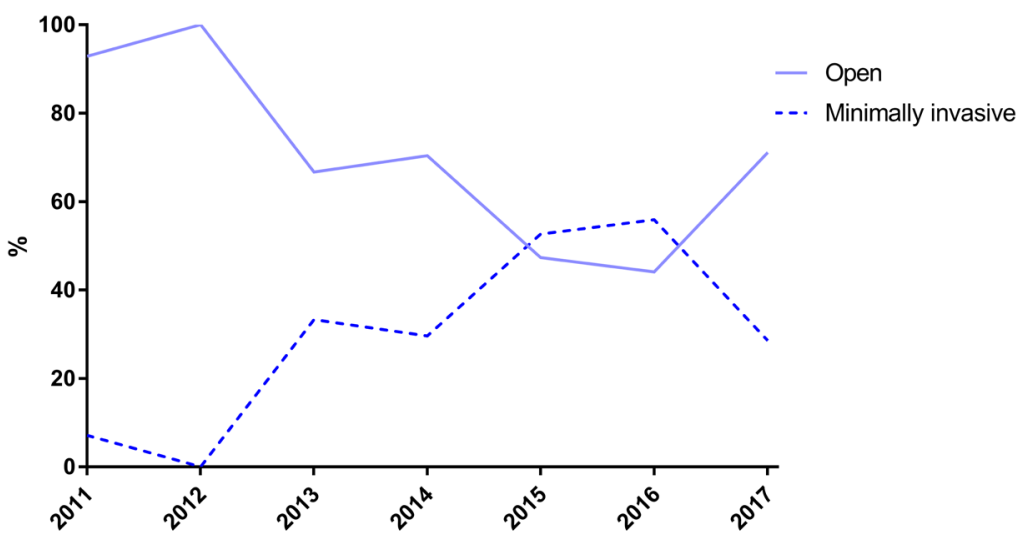

\begin{tabular}{|llllllll|}
\hline Numbers & & & & & & & \\
& 13 & 16 & 28 & 19 & 9 & 15 & 5 \\
Open & 1 & 0 & 14 & 8 & 10 & 19 & 2 \\
Minimally invasive & 1 &
\end{tabular}

B

Gastrectomies $(n=121)$
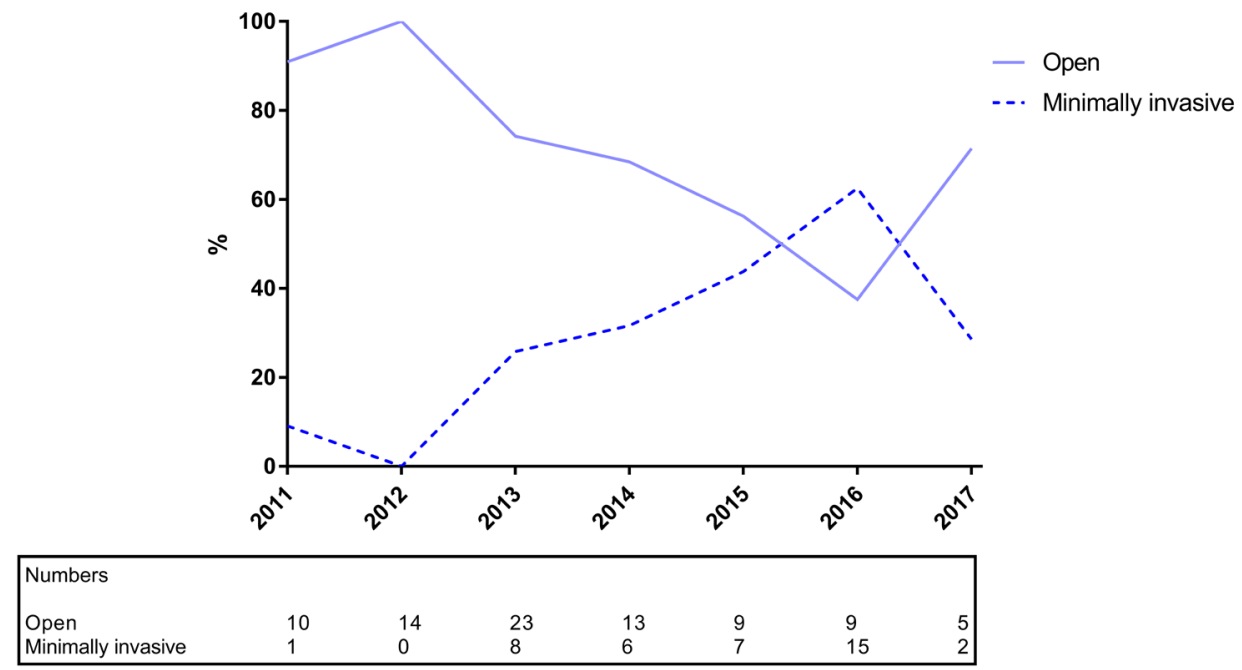

Figure 2. Minimally invasive and open surgical interventions (A) and gastrectomies (B) for acute presentation of gastric cancer in the study period. 
Table 3. Baseline characteristics of patients who underwent an acute surgical intervention for gastric cancer in the Netherlands between 2011 and 2017.

\begin{tabular}{|c|c|c|c|c|}
\hline \multirow[t]{2}{*}{ Characteristics } & \multicolumn{2}{|c|}{ Open } & \multicolumn{2}{|c|}{ Minimally invasive } \\
\hline & $\mathrm{n}=105$ & $\%$ & $\mathrm{n}=54$ & $\%$ \\
\hline \multicolumn{5}{|l|}{ Patient-related characteristics } \\
\hline Age, years $($ mean $\pm S D)$ & \multicolumn{2}{|c|}{$71 \pm 10$} & \multicolumn{2}{|c|}{$71 \pm 12$} \\
\hline \multicolumn{5}{|l|}{ Sex } \\
\hline Male & 79 & $75 \%$ & 33 & $61 \%$ \\
\hline Female & 26 & $25 \%$ & 21 & $39 \%$ \\
\hline BMI, $\mathbf{k g} / \mathbf{m}^{2}($ mean $\pm \mathrm{SD})$ & \multicolumn{2}{|c|}{$25 \pm 4$} & \multicolumn{2}{|c|}{$25 \pm 4$} \\
\hline \multicolumn{5}{|l|}{ ASA classification } \\
\hline I & 8 & $8 \%$ & 2 & $4 \%$ \\
\hline II & 39 & $37 \%$ & 25 & $46 \%$ \\
\hline III & 46 & $44 \%$ & 23 & $43 \%$ \\
\hline IV & 7 & $7 \%$ & 4 & $7 \%$ \\
\hline $\mathrm{V}$ & 1 & $1 \%$ & 0 & $0 \%$ \\
\hline Not specified & 4 & $4 \%$ & 0 & $0 \%$ \\
\hline \multicolumn{5}{|l|}{ Comorbidities } \\
\hline Cardiac & 35 & $33 \%$ & 24 & $44 \%$ \\
\hline Vascular & 42 & $40 \%$ & 27 & $50 \%$ \\
\hline Diabetes & 22 & $21 \%$ & 10 & $19 \%$ \\
\hline Pulmonary & 23 & $22 \%$ & 11 & $20 \%$ \\
\hline $\begin{array}{l}\text { Previous abdominal or thoracic } \\
\text { surgery }\end{array}$ & 37 & $35 \%$ & 18 & $33 \%$ \\
\hline \multicolumn{5}{|l|}{ Tumor location } \\
\hline Fundus & 6 & $6 \%$ & 3 & $6 \%$ \\
\hline Corpus & 28 & $27 \%$ & 12 & $22 \%$ \\
\hline Antrum & 48 & $46 \%$ & 22 & $41 \%$ \\
\hline Pylorus & 12 & $11 \%$ & 12 & $22 \%$ \\
\hline Stomach & 3 & $3 \%$ & 2 & $4 \%$ \\
\hline Other/not specified & 8 & $8 \%$ & 3 & $6 \%$ \\
\hline \multicolumn{5}{|l|}{ Histology } \\
\hline Adenocarcinoma & 88 & $84 \%$ & 49 & $91 \%$ \\
\hline Other & 8 & $8 \%$ & 4 & $7 \%$ \\
\hline Not specified & 9 & $8 \%$ & 1 & $2 \%$ \\
\hline \multicolumn{5}{|l|}{ cT status ${ }^{*}$} \\
\hline $\mathrm{T} 1$ & 4 & $4 \%$ & 2 & $4 \%$ \\
\hline $\mathrm{T} 2$ & 8 & $8 \%$ & 8 & $15 \%$ \\
\hline $\mathrm{T} 3$ & 32 & $30 \%$ & 20 & $37 \%$ \\
\hline $\mathrm{T} 4$ & 18 & $17 \%$ & 10 & $19 \%$ \\
\hline $\mathrm{Tx}$ & 43 & $41 \%$ & 13 & $24 \%$ \\
\hline \multicolumn{5}{|l|}{ cN status ${ }^{\star}$} \\
\hline N0 & 26 & $25 \%$ & 19 & $35 \%$ \\
\hline $\mathrm{N}+$ & 46 & $44 \%$ & 28 & $52 \%$ \\
\hline $\mathrm{Nx}$ & 33 & $31 \%$ & 7 & $13 \%$ \\
\hline
\end{tabular}


Table 3. Continued

\begin{tabular}{|c|c|c|c|c|}
\hline \multirow[t]{2}{*}{ Characteristics } & \multicolumn{2}{|c|}{ Open } & \multicolumn{2}{|c|}{ Minimally invasive } \\
\hline & $\mathrm{n}=105$ & $\%$ & $\mathrm{n}=54$ & $\%$ \\
\hline \multicolumn{5}{|l|}{ cM status $^{*}$} \\
\hline M0 & 76 & $72 \%$ & 44 & $81 \%$ \\
\hline M1 & 9 & $9 \%$ & 4 & $7 \%$ \\
\hline $\mathrm{Mx}$ & 20 & $19 \%$ & 6 & $11 \%$ \\
\hline \multicolumn{5}{|l|}{ Treatment-related characteristics } \\
\hline \multicolumn{5}{|l|}{ Neoadjuvant therapy ${ }^{\dagger}$} \\
\hline None & 93 & $89 \%$ & 52 & $96 \%$ \\
\hline Chemotherapy & 11 & $10 \%$ & 2 & $4 \%$ \\
\hline Chemoradiotherapy & 1 & $1 \%$ & 0 & $0 \%$ \\
\hline \multicolumn{5}{|l|}{ Setting } \\
\hline Emergent ( $<12$ hours) & 37 & $35 \%$ & 8 & $15 \%$ \\
\hline Urgent (>12 hours) & 68 & $65 \%$ & 46 & $85 \%$ \\
\hline \multicolumn{5}{|l|}{ Reason for acute surgery } \\
\hline Bleeding & 41 & $39 \%$ & 26 & $48 \%$ \\
\hline Perforation & 17 & $16 \%$ & 2 & $4 \%$ \\
\hline Other & 47 & $45 \%$ & 26 & $48 \%$ \\
\hline \multicolumn{5}{|l|}{ Surgical procedure } \\
\hline Total gastrectomy & 26 & $25 \%$ & 8 & $15 \%$ \\
\hline Partial gastrectomy & 57 & $54 \%$ & 31 & $57 \%$ \\
\hline Bypass (gastroenterostomy) & 17 & $16 \%$ & 9 & $17 \%$ \\
\hline Diagnostic laparotomy/laparoscopy & 5 & $5 \%$ & 6 & $11 \%$ \\
\hline \multicolumn{5}{|l|}{ Intention } \\
\hline Curative intent & 74 & $70 \%$ & 43 & $80 \%$ \\
\hline Palliative intent & 26 & $25 \%$ & 8 & $15 \%$ \\
\hline Not specified & 5 & $5 \%$ & 3 & $6 \%$ \\
\hline Lymph node dissection & 64 & $61 \%$ & 35 & $65 \%$ \\
\hline \multicolumn{5}{|l|}{ Year of surgery } \\
\hline $2011-2013$ & 57 & $54 \%$ & 15 & $28 \%$ \\
\hline $2014-2017$ & 48 & $46 \%$ & 39 & $72 \%$ \\
\hline
\end{tabular}

ASA American Society of Anesthesiologists; BMI body mass index at diagnosis; SD standard deviation Note. Data are numbers of patients with column-based percentages in parentheses, unless otherwise stated.

* Clinical T status and N status are based on AJCC TNM 7th edition.

${ }^{\dagger}$ The standard regimen for peri-operative treatment for gastro-esophageal junction or gastric adenocarcinoma generally consists of chemotherapy regimens similar to the MAGIC-trial (epirubicin, cisplatin and capecitabine). 
Table 4. Short-term outcomes for patients who underwent an acute gastrectomy for gastric cancer in the Netherlands between 2011 and 2017.

\begin{tabular}{|c|c|c|c|c|}
\hline & \multicolumn{2}{|c|}{ Open } & \multicolumn{2}{|c|}{ Minimally invasive } \\
\hline & $\mathrm{n}=\mathbf{8 3}$ & $\%$ & $\mathrm{n}=39$ & $\%$ \\
\hline \multicolumn{5}{|l|}{ Peroperative outcomes } \\
\hline Conversion & NA & & 9 & $23 \%$ \\
\hline Reasons for conversion & NA & & & \\
\hline Tumor extent & & & 4 & $10 \%$ \\
\hline Poor exposure & & & 4 & $10 \%$ \\
\hline Peroperative complication & & & 1 & $3 \%$ \\
\hline \multicolumn{5}{|l|}{ Postoperative complications } \\
\hline All $^{*}$ & 42 & $51 \%$ & 14 & $36 \%$ \\
\hline \multicolumn{5}{|l|}{ Medical complications } \\
\hline Pulmonary $\dagger$ & 15 & $18 \%$ & 5 & $13 \%$ \\
\hline Cardiac $\ddagger$ & 7 & $8 \%$ & 2 & $5 \%$ \\
\hline Thromboembolic\$ & 4 & $5 \%$ & 2 & $5 \%$ \\
\hline Neurologic & 4 & $5 \%$ & 2 & $5 \%$ \\
\hline Urologic\# & 4 & $5 \%$ & 1 & $3 \%$ \\
\hline \multicolumn{5}{|l|}{ Intra-abdominal complications } \\
\hline Anastomotic leakage ${ }^{\star \star}$ & 7 & $8 \%$ & 3 & $8 \%$ \\
\hline Abscess & 4 & $5 \%$ & 1 & $3 \%$ \\
\hline Chyle leakage & 0 & $0 \%$ & 0 & $0 \%$ \\
\hline \multicolumn{5}{|l|}{ Wound complications } \\
\hline Fascia dehiscence & 2 & $2 \%$ & 0 & $0 \%$ \\
\hline Wound infection $\dagger \dagger$ & 11 & $13 \%$ & 0 & $0 \%$ \\
\hline Other & 11 & $13 \%$ & 5 & $5 \%$ \\
\hline \multicolumn{5}{|l|}{ Reinterventions } \\
\hline All $^{*}$ & 12 & $14 \%$ & 9 & $23 \%$ \\
\hline Surgical & 11 & $13 \%$ & 6 & $15 \%$ \\
\hline Radiologic & 3 & $4 \%$ & 2 & $5 \%$ \\
\hline Endoscopic & 2 & $2 \%$ & 1 & $3 \%$ \\
\hline Not specified & 0 & $0 \%$ & 1 拉 & $3 \%$ \\
\hline \multicolumn{5}{|l|}{ Causes for reinterventions } \\
\hline Anastomotic leakage & 5 & $6 \%$ & 2 & $5 \%$ \\
\hline Fascia dehiscence & 1 & $1 \%$ & 0 & $0 \%$ \\
\hline $\begin{array}{l}\text { Complication of feeding } \\
\text { jejunostomy }\end{array}$ & 0 & $0 \%$ & 1 & $3 \%$ \\
\hline No complication encountered & 3 & $4 \%$ & 2 & $5 \%$ \\
\hline Other & 3 & $4 \%$ & 3 & $8 \%$ \\
\hline \multicolumn{5}{|l|}{ Recovery } \\
\hline ICU duration (median, IQR) & 1 & $(0-2)$ & 1 & $(0-1)$ \\
\hline Length of stay (median, IQR) & 11 & $(7-17)$ & 9 & $(7-16)$ \\
\hline Postoperative mortality $₫ \S$ & 9 & $11 \%$ & 3 & $8 \%$ \\
\hline Readmission to hospital $\mathbf{g g}$ & 9 & $11 \%$ & 3 & $8 \%$ \\
\hline
\end{tabular}


Table 4. Continued

\begin{tabular}{lrrrrr}
\hline & \multicolumn{2}{c}{ Open } & & \multicolumn{2}{c}{ Minimally invasive } \\
\cline { 5 - 6 } \cline { 5 - 5 } Oncological outcomes & $\mathbf{n}=\mathbf{8 3}$ & & & $\mathbf{n}=\mathbf{3 9}$ & \% \\
\hline Radicality & & & & \\
$\quad$ R0 & 55 & $66 \%$ & 34 & $87 \%$ \\
R1 & 14 & $17 \%$ & 3 & 0 & $0 \%$ \\
R2 & 11 & $13 \%$ & 21 & $(15-32)$ \\
Lymph node yield (median, IQR) & 16 & $(11-24)$ & 5 & $(1-10)$ \\
Positive lymph nodes harvested & 4 & $(0-9)$ & & \\
(median, IQR) & & & & \\
\hline
\end{tabular}

$I Q R$ interquartile range, $N A$ not applicable

Note. Missing values were encountered in ICU duration $(n=12)$, length of stay $(n=2)$, readmission $(n=4)$, radicality $(n=5)$, lymph node yield $(n=2)$ and positive lymph nodes harvested $(n=2)$.

* Defined as number of patients who experienced a postoperative complication or underwent a reintervention. One patient could experience more than 1 complication or reintervention.

${ }^{\dagger}$ Pneumonia, pleural effusion, respiratory failure, pneumothorax and/or acute respiratory distress syndrome.

* Supraventricular and ventricular arrhythmia, myocardial infarction and/or heart failure.

${ }^{\varsigma}$ Pulmonary embolism, deep venous thrombosis, stroke and/or thrombophlebitis.

'Acute delirium.

* Urinary tract infection, urinary retention and/or renal insufficiency.

${ }^{* *}$ Any clinically or radiologically proven anastomotic leakage.

${ }^{\dagger \dagger}$ Wound infection requiring drainage or antibiotic treatment.

* Patient who underwent a reintervention for a complication of the feeding jejunostomy.

$\$$ Death during initial hospital admission or within 30 days after surgery.

"Readmission to hospital within 30 days after initial discharge.

\section{Discussion}

In this nationwide cohort study concerning patients who underwent acute surgery for esophageal and gastric cancer, short-term postoperative and oncological outcomes of minimally invasive resections were comparable to open resections.

Previous studies have shown the safety and feasibility of minimally invasive surgery for esophagogastric cancer in the elective setting. ${ }^{3,4,12,11,5-10}$ For esophageal cancer, minimally invasive esophagectomy resulted in a shorter hospital stay, higher lymph node yield, similar radical resection rate and postoperative pulmonary complications, but higher reintervention rates in several population-based studies. ${ }^{5-8}$ Due to the low number of acute esophagectomies, it was unfortunately not possible in this study to perform the analyses that would be required to reproduce these results for minimally invasive esophagectomy in the acute setting. For gastric cancer, minimally invasive gastrectomy in the elective setting was deemed to be safe and feasible regarding overall postoperative morbidity and mortality rates, as well as short-term oncological outcomes, and resulted in decreased wound complications and a shorter hospital stay compared to open gastrectomy. ${ }^{12,10}$ The current study of minimally invasive gastrectomy in the acute setting also demonstrated comparable 
oncological outcomes to the open approach, as well as a potentially decreased median length of hospital stay. However, the current study demonstrated a higher conversion rate of minimally invasive gastrectomies in the acute setting (23\% versus $0.9 \%^{12}, 3.5 \%{ }^{11}$ and $10 \%{ }^{10}$ in the elective setting), as well as a potentially increased percentage of patients that underwent a reintervention after minimally invasive gastrectomy (23\% versus $14 \%$ in the acute setting and $0.4 \%$ versus $0.4 \%{ }^{11}, 1.2 \%$ versus $1.5 \%{ }^{12}$ and $17 \%$ versus $16 \%{ }^{10}$ in the elective setting for minimally invasive gastrectomy versus open gastrectomy, respectively). Unfortunately no clear explanation for this difference could be deduced from the causes for the reinterventions as available in the data.

In order to correctly interpret the current results, it must be noted that esophagogastric cancer surgery has been centralized since 2011 in the Netherlands. This is one of the main reasons that $84 \%$ of the elective esophagectomies and $40 \%$ of the elective gastrectomies are performed by minimally invasive techniques in the recent years. ${ }^{6,9}$ A similar increase has been seen for the use of minimally invasive techniques in the acute setting. Most surgeons in the Netherlands implemented these techniques after participating in a hands-on course on minimally invasive esophagectomy and gastrectomy, followed by several cases with an experienced surgeon present. The influence of centralization and learning curves on postoperative outcomes also seem to be important in for esophagogastric surgery in the acute setting, as demonstrated by a recently published study from England. ${ }^{16}$ High-volume cancer centers and surgeons are more experienced in managing patients following esophagectomy and gastrectomy, and have the appropriate infrastructure available. ${ }^{16}$ As such, they might be better equipped to deliver consistent levels of high-quality outcomes for minimally invasive surgery in the acute setting as well which might explain the comparable outcomes for minimally invasive and open surgery in the current study.

Overall, acute surgical interventions for upper gastrointestinal malignancies are rare, accounting for only $2 \%$ of all surgical interventions for upper gastrointestinal malignancies in the Netherlands. This especially applies for surgical interventions for esophageal cancer, occurring approximately once yearly in a country with an incidence of approximately 2500 new esophageal cancer cases per year. ${ }^{17}$ Interestingly, most patients who underwent an acute surgical intervention had prior histological confirmation of their cancer diagnosis, indicating that patients generally did not present with an acute symptom of an unknown malignancy. The majority of the gastric cancer cases had a cause for acute intervention other than bleeding or perforation. As the DUCA registry only registers the indication for acute intervention in 3 prespecified categories (bleeding, perforation and other), the frequency of obstruction as another important indication for acute surgical interventions in gastric cancer could not be researched. However, in patients with an obstruction, a gastroenterostomy or distal gastrectomy might have been more frequently performed. When the surgical procedures of patients without a specified reason for the surgical 
intervention are explored in more detail, it might indeed be that obstruction accounts for a large share of the not specified indications, as 30\% of them underwent a gastroenterostomy (21/71) and 44\% underwent a partial gastrectomy (31/71) (data not shown).

The population-based design with virtually complete inclusion of all patients in the Netherlands is a significant strength of the study, along with the prospective data collection and detailed information on strictly defined postoperative outcomes. However, there are some limitations of the current study that need to be addressed. First, the small numbers of patients in all groups precluded a direct comparison between minimally invasive and open surgery correcting for bias. This prevents firm conclusions to be drawn regarding the potentially observed benefits of minimally invasive compared to open surgery in the acute setting (e.g. the decreased median length of hospital stay, higher observed percentage of radical resections and increased median lymph node yield), as well as regarding the potential disadvantages of minimally invasive surgery (e.g. the increased percentage of reinterventions after minimally invasive gastrectomy compared to open gastrectomy). Second, the introduction of minimally invasive surgery for upper gastrointestinal malignancies occurred simultaneously with centralization of cancer care and the introduction of enhanced recovery after surgery (ERAS) programs in the Netherlands. It has been shown that centralization of surgery is associated with reduced complications and improved long-term survival ${ }^{18-21}$ and that the use of ERAS programs protocols can reduce the length of hospital stay. ${ }^{22,23}$ As such, the observed outcomes in this study for both minimally invasive as open surgery are probably influenced by these factors. It must be further acknowledged as a limitation that, due to the privacy restrictions of the national database, individual hospital related factors such as postoperative management protocols and background experience in minimally invasive surgery might have influenced the results but were not available. This also applies to more detailed information regarding the reasons for surgical intervention, such as iatrogenic versus spontaneous perforations and the severity of the bleeding. Lastly, no long-term survival data is available for the patients in the DUCA registry.

Future research regarding this topic would benefit from an even larger cohort study that would allow for statistical analyses corrected for bias to compare minimally invasive surgery and open surgery for esophagogastric cancer in the acute setting. However, considering the rarity of these events, larger case series are probably difficult to find.

In conclusion, this nationwide cohort study demonstrates that acute surgical interventions for esophageal and gastric cancer are rare. For gastric cancer, minimally invasive surgery appears to be feasible and safe in the acute setting with at least comparable postoperative clinical and short-term oncological outcomes compared to open surgery, but a relatively high conversion rate. 


\section{References}

1. Haverkamp L, Seesing MF, Ruurda JP, Boone J, V Hillegersberg R. Worldwide trends in surgical techniques in the treatment of esophageal and gastroesophageal junction cancer. Dis esophagus. 2017;30(1):1-7.

2. Brenkman HJ, Haverkamp L, Ruurda JP, van Hillegersberg R. Worldwide practice in gastric cancer surgery. World J Gastroenterol. 2016;22(15):4041-4048.

3. Biere SS, van Berge Henegouwen MI, Maas KW, et al. Minimally invasive versus open oesophagectomy for patients with oesophageal cancer: a multicentre, open-label, randomised controlled trial. Lancet. 2012;379(9829):1887-1892.

4. Van Der Sluis PC, Van Der Horst S, May AM, et al. Robot-assisted Minimally Invasive Thoracolaparoscopic Esophagectomy Versus Open Transthoracic Esophagectomy for Resectable Esophageal Cancer A Randomized Controlled Trial. Trials 2018.

5. Sihag S, Kosinski AS, Gaissert HA, Wright CD, Schipper PH. Minimally Invasive Versus Open Esophagectomy for Esophageal Cancer: A Comparison of Early Surgical Outcomes From The Society of Thoracic Surgeons National Database. Ann Thorac Surg. 2016;101(4):1281-1289.

6. Seesing MFJ, Gisbertz SS, Goense L, et al. A Propensity Score Matched Analysis of Open Versus Minimally Invasive Transthoracic Esophagectomy in the Netherlands. Ann Surg. 2017;266(5):839-846.

7. Takeuchi H, Miyata H, Ozawa S, et al. Comparison of Short-Term Outcomes Between Open and Minimally Invasive Esophagectomy for Esophageal Cancer Using a Nationwide Database in Japan. Ann Surg Oncol. 2017;24(7):1821-1827.

8. Mamidanna R, Bottle A, Aylin P, Faiz O, Hanna GB. Short-term outcomes following open versus minimally invasive esophagectomy for cancer in England: a population-based national study. Ann Surg. 2012;255(2):197-203.

9. Brenkman HJF, Ruurda JP, Verhoeven RHA, van Hillegersberg R. Safety and feasibility of minimally invasive gastrectomy during the early introduction in the Netherlands: short-term oncological outcomes comparable to open gastrectomy. Gastric cancer. 2017;20(5):853-860.

10. Brenkman HJF, Gisbertz SS, Slaman AE, et al. Postoperative Outcomes of Minimally Invasive Gastrectomy Versus Open Gastrectomy During the Early Introduction of Minimally Invasive Gastrectomy in the Netherlands: A Population-based Cohort Study. Ann Surg. 2017;266(5):831-838.

11. Katai H, Mizusawa J, Katayama H, et al. Short-term surgical outcomes from a phase III study of laparoscopyassisted versus open distal gastrectomy with nodal dissection for clinical stage IA/IB gastric cancer: Japan Clinical Oncology Group Study JCOG0912. Gastric Cancer. 2017;20(4):699-708.

12. Kim W, Kim H-H, Han S-U, et al. Decreased Morbidity of Laparoscopic Distal Gastrectomy Compared With Open Distal Gastrectomy for Stage I Gastric Cancer. Ann Surg. 2016;263(1):28-35.

13. Busweiler LAD, Wijnhoven BPL, van Berge Henegouwen MI, et al. Early outcomes from the Dutch Upper Gastrointestinal Cancer Audit. Br J Surg. 2016;103(13):1855-1863.

14. National guideline esophageal cancer (version 3.1). 2015. http://www.oncoline.nl/oesofaguscarcinoom. Accessed March 21, 2018.

15. National guideline gastric cancer (version 2.2). 2017. https://www.oncoline.nl/maagcarcinoom. Accessed March 21, 2018.

16. Markar SR, Mackenzie H, Wiggins T, et al. Influence of national centralization of oesophagogastric cancer on management and clinical outcome from emergency upper gastrointestinal conditions. Br J Surg. 2018;105(1):113-120.

17. Integraal Kankercentrum Nederland (IKNL). Cijfers over kanker. 2018. https://www.cijfersoverkanker.nl/. Accessed December 11, 2018.

18. Coupland VH, Lagergren J, Lüchtenborg M, et al. Hospital volume, proportion resected and mortality from oesophageal and gastric cancer: a population-based study in England, 2004-2008. Gut. 2013;62(7):961966.

19. Derogar M, Sadr-Azodi O, Johar A, Lagergren P, Lagergren J. Hospital and Surgeon Volume in Relation to Survival After Esophageal Cancer Surgery in a Population-Based Study. J Clin Oncol. 2013;31(5):551-557.

20. Brusselaers N, Mattsson F, Lagergren J. Hospital and surgeon volume in relation to long-term survival after oesophagectomy: systematic review and meta-analysis. Gut. 2014;63(9):1393-1400. 
21. Dikken JL, Dassen AE, Lemmens VEP, et al. Effect of hospital volume on postoperative mortality and survival after oesophageal and gastric cancer surgery in the Netherlands between 1989 and 2009. Eur J Cancer. 2012;48(7):1004-1013.

22. Findlay JM, Gillies RS, Millo J, Sgromo B, Marshall RE, Maynard ND. Enhanced recovery for esophagectomy: a systematic review and evidence-based guidelines. Ann Surg. 2014;259(3):413-431.

23. Markar SR, Schmidt H, Kunz S, Bodnar A, Hubka M, Low DE. Evolution of standardized clinical pathways: refining multidisciplinary care and process to improve outcomes of the surgical treatment of esophageal cancer. J Gastrointest Surg. 2014;18(7):1238-1246. 

PART IV

Summary and general discussion 


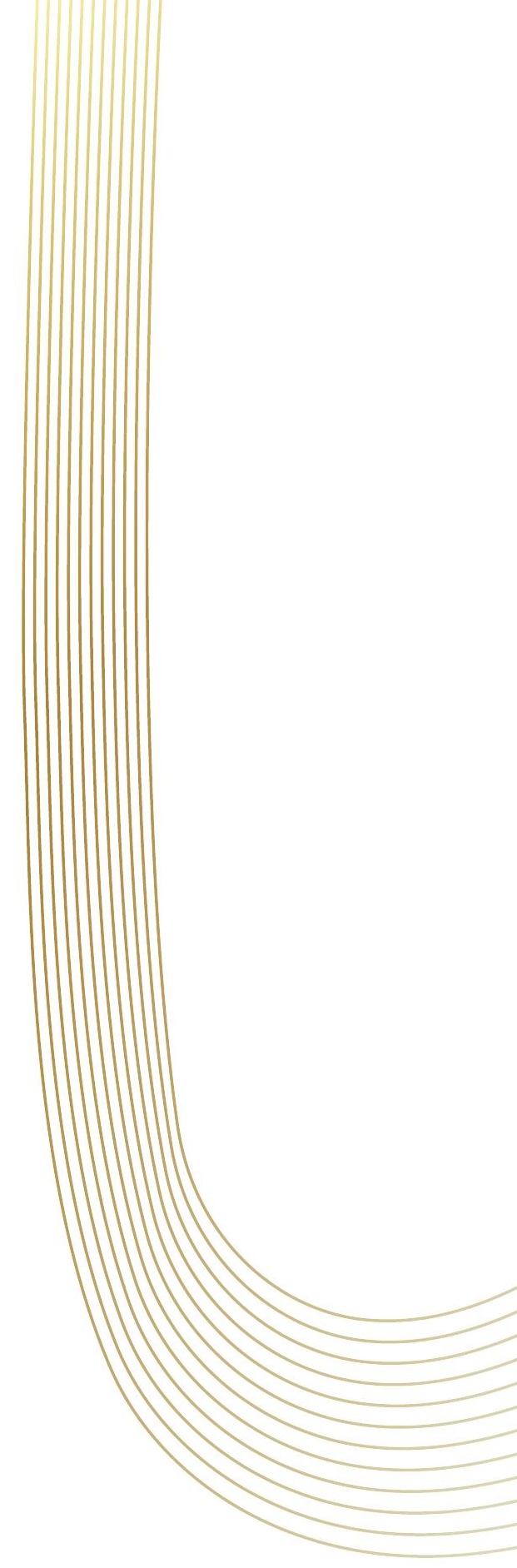


Chapter 13

Summary 
Esophageal cancer affects almost 600000 people per year worldwide. Although the prognosis of patients with esophageal cancer has improved over the past decades, treatment still has a detrimental impact on a patients' quality of life. Personalized medicine is rapidly gaining popularity for esophageal cancer, but largely remains a philosophy rather than clinical practice due to limited evidence on accurate treatment response assessment and personalized treatment strategies. As such, the research presented in this thesis aimed at improvements in neoadjuvant chemoradiotherapy (part I), treatment response assessment (part II) and surgical treatment (part III) in patients with esophageal cancer.

\section{Neoadjuvant chemoradiotherapy}

Part I of this thesis focused on optimizing treatment efficacy rates of neoadjuvant chemoradiotherapy while balancing the toxicities, and provided insight in the consequences of toxicities of neoadjuvant chemoradiotherapy for the patient.

Chapter 2 described the impact of neoadjuvant chemoradiotherapy and related adverse events on short-term health-related quality of life. The negative effect of neoadjuvant chemoradiotherapy appeared to be most pronounced on physical functioning and fatigue, whereas a positive effect was observed in odynophagia at 1 month follow-up. General health-related quality of life remained similar. Adverse events during neoadjuvant chemoradiotherapy had a negative effect on social functioning, dysphagia, pain and taste at follow-up. This information could be used by physicians to adequately manage patients' expectations about their quality of life during neoadjuvant chemoradiotherapy, so that patients can anticipate on these effects and take well informed treatment decisions.

As a first step towards minimizing toxicity rates of neoadjuvant chemoradiotherapy by means of real-time tumor tracking and personalized tumor margins during radiotherapy, chapter 3 researched variations in intra-fraction motion. Patients undergoing neoadjuvant chemoradiotherapy for esophageal cancer underwent 6 sequential MRI scans. To quantify intra-fraction motion, 2 cine-MRI series were acquired with approximately 10 minutes interval, both in coronal and sagittal plane. Intra-fraction motion was found to be highly variable between and within patients with esophageal cancer during neoadjuvant chemoradiotherapy. The stochastic nature of the tumor drift indicated that real-time tumor motion management during radiotherapy is a prerequisite to safely reduce radiotherapy margins.

The multi-institutional analysis in chapter 4 researched whether neoadjuvant radiation dose increases pathologic response rates in esophageal cancer patients. A possible strategy for treatment intensification could be to increase the neoadjuvant radiation dose and the available studies that assess whether neoadjuvant radiation dose influences tumor response 
rates are equivocal. No radiation dose response relation was observed for pathologic complete response. However, pathologic major response (defined as patients with a pathologic complete response and patients with a near-complete response) might be impacted by neoadjuvant radiation dose. Further prospective trials are needed to evaluate a potential survival benefit.

\section{Treatment response assessment}

Accurate assessment of response to neoadjuvant chemoradiotherapy is important for safe implementation of an organ-sparing approach in pathologic complete responders, but also to adapt treatment strategies in expected poor responders. Studies researching the performance of a multimodality imaging approach for treatment response assessment were presented in part II of this thesis.

Chapter 5 described the distinct and combined value of ${ }^{18} \mathrm{~F}$-fluorodeoxyglucose positron emission tomography with integrated computed tomography $\left({ }^{18} \mathrm{~F}-\mathrm{FDG}\right.$ PET-CT) and diffusion-weighted (DW) magnetic resonance imaging (MRI) for treatment response prediction. This prospective international multicenter study demonstrated that changes on ${ }^{18} \mathrm{~F}-\mathrm{FDG}$ PET/CT after neoadjuvant chemoradiotherapy and early changes on DW-MRI during neoadjuvant chemoradiotherapy can help identify pathologic complete responders to nCRT in esophageal cancer. Moreover, ${ }^{18} \mathrm{~F}-\mathrm{FDG}$ PET/CT and DW-MRI might be of complementary value in the assessment of pathologic complete responders. When combining both modalities, patients with a pathologic complete response could be accurately discriminated from patients without a pathologic complete response in $83 \%$ of the patients (c-statistic: 0.83 ).

The potential of DW-MRI for treatment response assessment was further discussed in chapter 6. In this prospective study, patients underwent 6 DW-MRI scans: 1 baseline scan before starting neoadjuvant chemoradiotherapy and weekly scans during 5 weeks of neoadjuvant chemoradiotherapy with the aim to establish the optimal timing of DW-MRI scanning during neoadjuvant chemoradiotherapy for the prediction of pathologic complete response. The relative change in tumor apparent diffusion coefficient $(\triangle \mathrm{ADC}(\%))$ as measured on DW-MRI during the first 2 weeks of neoadjuvant chemoradiotherapy was the most predictive for pathologic complete response. These results could further aid the minimization of the burden of repeated scanning for patients, as well as optimal usage of the available (financial) resources, while assuring the best predictive ability of DW-MRI.

Chapter 7 provides the rationale and study protocol for a nationwide, prospective study with the aim to develop a multimodal prediction model that predicts the patients' individual probability of a pathologic complete response after neoadjuvant chemoradiotherapy for 
esophageal cancer. In this observational trial (PRIDE study) recruiting 200 patients, patients will undergo $3{ }^{18} \mathrm{~F}$-FDG PET-CT scans and $3 \mathrm{MRI}$ scans before, during and after treatment with neoadjuvant chemoradiotherapy. Additionally, blood samples are obtained at the same 3 time points for the analysis of the presence of circulating tumor DNA (ctDNA) and an endoscopy with biopsies as well as an endoscopic ultrasonography after finishing neoadjuvant chemoradiotherapy. Accurate prediction of response to neoadjuvant chemoradiotherapy could lead to a patient-tailored approach with omission of surgery in the future in case of predicted pathologic complete response, potentially improving quality of life and reducing health care costs. Furthermore, additional neoadjuvant treatment might be offered to poor responders.

Lastly, chapter 8 evaluated the burden of repeated MRI and PET-CT scanning during neoadjuvant chemoradiotherapy from the perspective of the patient. In this study, the experienced burden of repeated MRI and PET-CT examinations during neoadjuvant treatment was evaluated with a self-report questionnaire. Few patients experienced discomfort, pain, anxiety, and embarrassment during MRI and PET-CT. When asked to rank different examinations, patients preferred MRI over PET-CT. As such, both MRI and PET-CT are generally well-tolerated from a patient perspective and could be used for the assessment of response to treatment in esophageal cancer patients.

\section{Surgical treatment}

Part III of this thesis addressed the surgical treatment of esophageal cancer patients in the era of multimodality management.

The narrative review in chapter 9 provides an extensive outline of the literature on surgical treatment in the era of multimodality management of esophageal cancer. Although esophagectomy is still considered the cornerstone of curative treatment for locally advanced esophageal cancer, it remains associated with considerable postoperative morbidity, despite promising results of minimally invasive techniques. In this light, both physical status and response to neoadjuvant therapy are important factors for selecting patients who will benefit from surgery. Furthermore, it is important to optimize the entire perioperative trajectory: from the initial outpatient clinic visit to postoperative discharge. Enhanced Recovery After Surgery (ERAS) is increasingly recognized for esophagectomy and emphasizes perioperative aspects such as nutrition, physiotherapy, and pain management.

Despite its prognostic benefits, neoadjuvant chemoradiotherapy for resectable esophageal cancer is accompanied by the risk of treatment-associated toxicities. This is especially relevant when these toxic effects prevent patients from undergoing surgical resection. By means of a population-based study including all patients from the Netherlands who started 
neoadjuvant chemoradiotherapy for esophageal cancer in 2015-2016 as described in chapter 10, it was demonstrated that 1 in 7 patients who started neoadjuvant chemoradiotherapy eventually does not proceed to surgical resection. These patients have a lower overall survival compared to the patients who undergo neoadjuvant chemoradiotherapy followed by surgical resection. These findings could be a starting point for further research, which should aim to investigate whether this patient group can be selected prior to treatment, and whether patient-individualized treatment, centralization of oncological care and counseling will result in a larger proportion of patients who will undergo surgery.

Chapter 11 builds on previous research by our group concerning atherosclerotic calcification as measured on routine diagnostic CT scans as a risk factor for the development of anastomotic leakage - a severe complication - following esophagectomy. In order to study whether this association derived from local flow limitations or vascular disease in general, diagnostic CT images of esophageal cancer patients were scored for the presence of arterial calcification on 10 locations based on a visual grading system and related to anastomotic leakage. Calcification of the coronary and supra-aortic arteries on routine CT were predictive of cervical anastomotic leakage after esophagectomy. These results suggested that generalized cardiovascular disease is a strong indicator for the risk of leakage. This new described risk factor may be used in future prediction models, and eventually aid to a more individualized identification of the risk of anastomotic leakage following esophagectomy.

With the rise of minimally invasive surgery in the elective setting, the safety and feasibility of minimally invasive esophagectomy and gastrectomy in the acute setting was assessed in chapter 12. This nationwide study including data from the Dutch UpperGI Cancer Audit (DUCA) between 2011 and 2017 demonstrated that acute resections for esophageal cancer were very rare. As these numbers were very low, no comparison between minimally invasive and open esophagectomies was made. Minimally invasive gastrectomy however was at least comparable to open gastrectomy regarding postoperative complications and oncological outcomes. As such, it was deemed safe and feasible in the acute setting.

In conclusion, the research presented in this thesis provides encouragement for individualized treatment for esophageal cancer patients by means of multimodality treatment response assessment using ${ }^{18} \mathrm{~F}-\mathrm{FDG}$ PET/CT and DW-MRI, balancing treatment efficacy and toxicity of neoadjuvant therapy, and more appropriately perform riskstratification of patients for surgery following neoadjuvant therapy. 


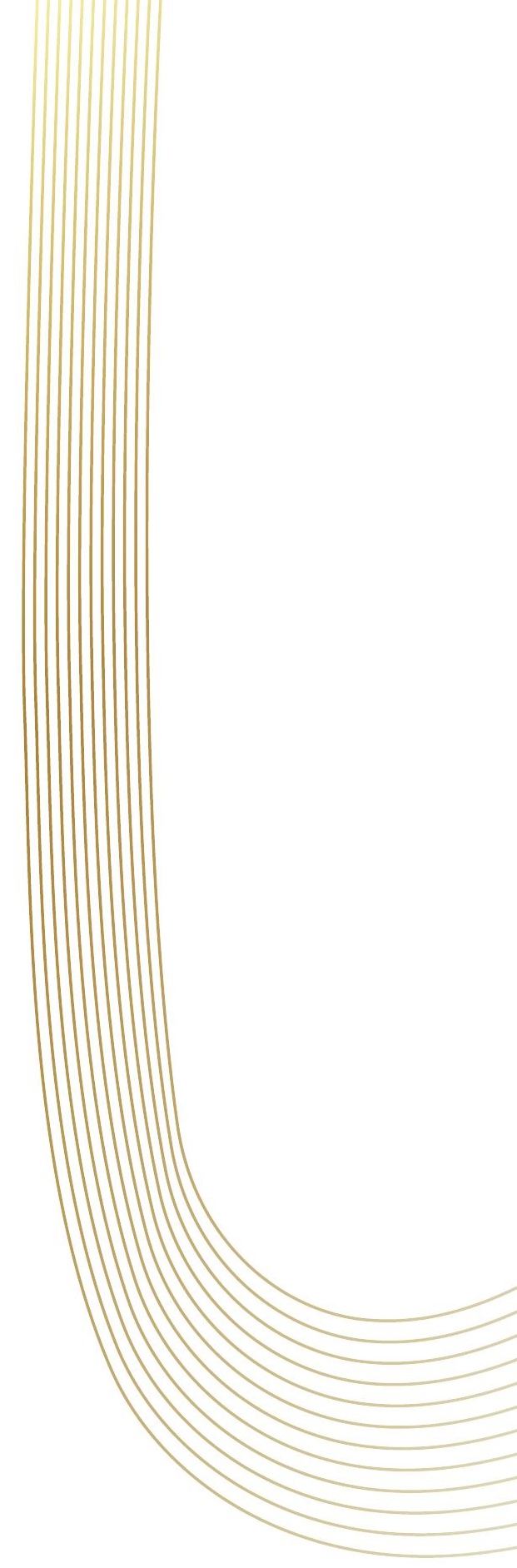


Chapter 14

General discussion and future perspectives 
The treatment of locally advanced esophageal cancer is increasingly shifting towards individualized strategies that are based on developments within the fields of neoadjuvant therapy and surgery. The aim of this thesis was to establish evidence to guide individualized decision-making for esophageal cancer patients by assessing the impact and efficacy of neoadjuvant chemoradiotherapy (part I), improving treatment response assessment (part II) and refining surgical treatment (part III). In this final chapter, the implications of the findings for current and future clinical practice are discussed.

\section{Neoadjuvant treatment}

In most countries, patients with locally advanced esophageal cancer undergo neoadjuvant chemoradiotherapy or chemotherapy before esophagectomy. Neoadjuvant chemoradiotherapy is the widely accepted treatment of choice for patients with esophageal squamous cell carcinoma, whereas the optimal multimodality regimen remains topic of debate for patients with adenocarcinoma. Hence, worldwide variation exists on the preferred neoadjuvant treatment strategy. While many European centers use neoadjuvant chemoradiotherapy (nCRT) according to the CROSS regimen (carboplatin/paclitaxel with concurrent $41.4 \mathrm{~Gy})^{1}$, neoadjuvant radiation dosages are generally higher (50.4 Gy) and combined with 5-fluorouracil and taxane or with platinum-based compounds in NorthAmerica. Neoadjuvant or perioperative chemotherapy regimens vary as well; the $\mathrm{OEO} 2^{2}$, MAGIC $^{3}$ and FNCLCC/FFCD ${ }^{4}$ regimens are all commonly used. This section discusses the relevant considerations in the search for the optimal neoadjuvant treatment strategy for esophageal cancer.

\section{Balancing benefits and toxicity}

Neoadjuvant treatment should ideally prolong survival while avoiding treatment-related burden. The survival benefit of neoadjuvant therapy in the treatment of locally advanced esophageal cancer has been repeatedly demonstrated, as both disease-free and overall survival were shown to increase when compared to surgery alone. ${ }^{5,6}$ Even so, treatmentrelated toxicity might impact quality of life and is therefore important to be considered. In this context, chapter 2 investigated the impact of neoadjuvant chemoradiotherapy on shortterm health-related quality of life (HRQOL) in patients undergoing curatively intended treatment for esophageal cancer, showing that impairments in HRQOL were generally limited and mostly involved physical functioning and fatigue, which is in line with previous research. ${ }^{7-9}$ However, in patients who suffered from adverse events during neoadjuvant chemoradiotherapy, a significant decline in HRQOL was observed. These findings emphasize the need to identify ways to reduce adverse events and toxicity during neoadjuvant treatment. 
Treatment-related toxicity of neoadjuvant chemoradiotherapy might be reduced by margin reduction during radiation therapy, aiming at avoiding radiation to surrounding organs at risk. Previous studies demonstrated that radiation of the heart and lungs during neoadjuvant treatment for esophageal cancer has a negative impact on overall survival. ${ }^{10} \mathrm{MRI}$-guided radiation delivery and proton therapy may improve targeted irradiation of the tumor while sparing surrounding organs at risk from high radiation dosages. ${ }^{11-13}$ These techniques have been recently introduced and are currently being investigated for several cancer types, including esophageal cancer. Some evidence has already been generated for the use of proton therapy in esophageal cancer: an observational cohort study reported comparable efficacy in combination with lower rates of treatment-related toxicity in comparison to photon therapy. ${ }^{14}$ Future studies will need to determine the potential clinical advantage of proton therapy and MRI-guided radiation delivery in terms of reducing treatment-related toxicity and improving treatment efficacy in the neoadjuvant treatment for esophageal cancer.

Next to reducing treatment-related toxicity, researchers should also strive to maximize the efficacy of neoadjuvant chemoradiotherapy. Radiation dose escalation might be interesting, particularly for the relatively large proportion of patients with a near-complete response after neoadjuvant chemoradiotherapy (19\%), as these patients have a decreased survival compared to pathologic complete responders. Hence, increasing pathologic complete response rates is an important aim of current research. Radiation dose escalation might be performed by means of simultaneous integrated boost techniques or sequential boost techniques. Studies using simultaneous integrated boost techniques have thus far demonstrated the potential of improved locoregional control rates in esophageal cancer. ${ }^{15,16}$ Sequential boost techniques might even be preferable, as these allow for response based dose-escalation. A recently published phase I trial established the feasibility and safety of a cumulative tumor dose of 86 Gy in locally advanced esophageal squamous cell carcinomas, using hyperfractionated radiation therapy following standard-dose chemoradiotherapy. ${ }^{17}$ Late esophageal toxicity was relatively infrequent and mild in this study ${ }^{17}$, which is in contrast to the findings of the RTOG 9207 study, which intensified radiotherapy with esophageal brachytherapy and reported an unexpectedly high incidence (14\%) of esophageal fistulas ( 1 aortoesophageal, 3 esophageal-bronchotracheal and 2 esophageal-mediastinal fistulas).$^{18}$ Future studies on radiation dose escalation in esophageal cancer should focus on partial responders to neoadjuvant chemoradiotherapy or patients with squamous cell carcinomas (as these are more radiosensitive than adenocarcinomas) in light of an organsparing approach for these patients. Non-responders and adenocarcinomas are more likely to benefit from alternative therapies instead of higher radiation dosages. 


\section{Alternatives to neoadjuvant chemoradiotherapy}

Alternative therapies to neoadjuvant chemoradiotherapy include perioperative chemotherapy and targeted therapies. The FLOT4 trial recently showed that perioperative chemotherapy with the FLOT regimen (docetaxel, oxaliplatin, leucovorin, and 5-FU) was associated with increased survival in patients with gastric or gastro-esophageal adenocarcinoma compared to the ECF/ECX triplet regimen (epirubicin, cisplatin, and 5-FU or capecitabine). ${ }^{19}$ It should be noted, however, that almost half of the included patients had gastric adenocarcinoma, which might imply that the results cannot be generalized to the esophageal cancer population. Several randomized trials are now comparing perioperative chemotherapy and neoadjuvant chemoradiotherapy for esophageal cancer (NeoAegis ${ }^{20}$, ESOPEC ${ }^{21}$, CMISG1701 ${ }^{22}$, NEOSCOPE ${ }^{23}$, PROTECT-1402 ${ }^{24}$ ), which are expected to provide more clarity in the near future.

Besides chemotherapy and radiotherapy, targeted anticancer therapies such as human epidermal growth factor receptor 2 (HER2) antibodies (trastuzumab, pertuzumab) and vascular endothelial growth factor receptor (VEGFR)-2 antibodies (ramucirumab) are emerging. Moreover, the addition of immunotherapeutic strategies to neoadjuvant chemoradiation or perioperative chemotherapy is currently being evaluated, such as programmed death ligand 1 (PD-L1) targeted therapy with atezolizumab or pembrolizumab. ${ }^{25}$ These immunotherapeutic strategies have great potential, but a much better understanding of the immune microenvironment underlying esophageal squamous cell and adenocarcinoma is needed if we are to exploit the inherent cancer fighting capabilities of each individual patient's immune system. ${ }^{25}$

Although surgery combined with adjuvant treatment might not be expected to yield survival benefits for the overall esophageal cancer population ${ }^{26}$, it might make sense to selectively administer adjuvant therapy to patients who have first been treated with neoadjuvant therapy followed by esophagectomy. As esophageal cancer patients with nodal metastases are likely at high risk for micrometastatic systemic disease and disease recurrence following surgical resection, it seems biologically plausible that adjuvant therapy could result in survival benefit in this subgroup. In patients with residual nodal disease (ypT0-4N+) following neoadjuvant chemoradiotherapy and esophagectomy, adjuvant chemo- or radiotherapy have been suggested to improve survival. ${ }^{27,28}$ However, these findings need to be confirmed, ideally in a formal randomized controlled trial, before routine application in clinical practice can be recommended.

The abovementioned considerations and suggestions for improvement of treatment efficacy and decreasing the burden of treatment have been summarized in Figure 1. 


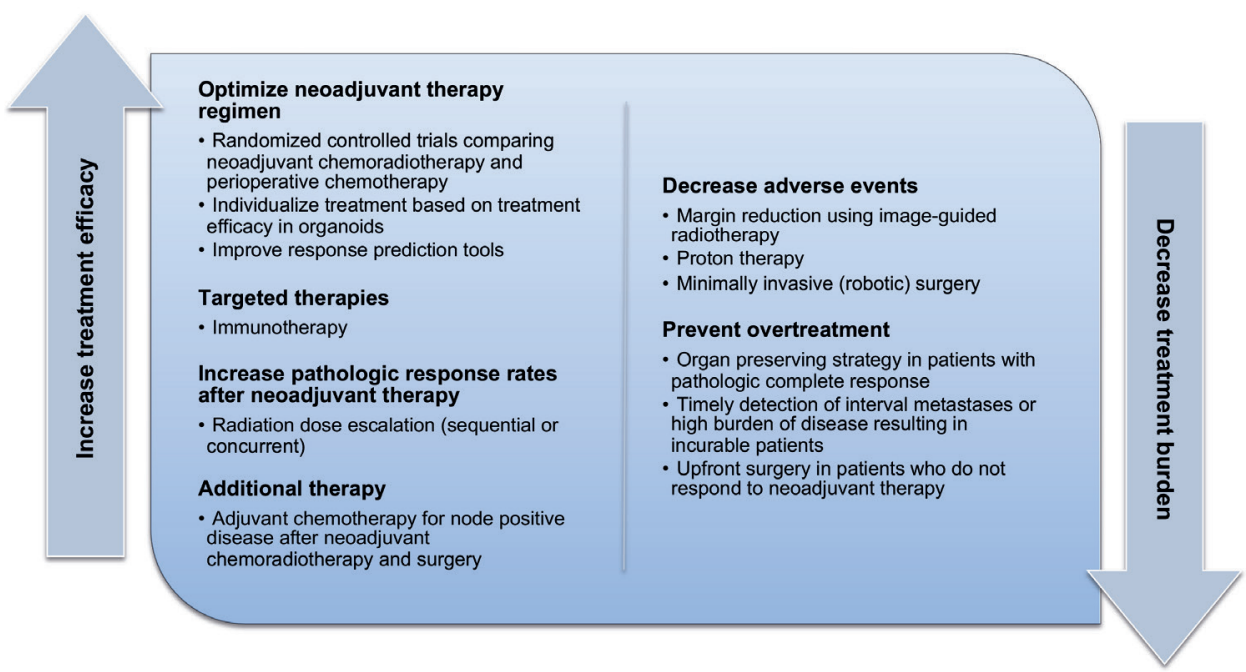

Figure 1. Summary of improvements to balance efficacy and burden of treatment in esophageal cancer patients

\section{Treatment response assessment}

Following neoadjuvant chemoradiotherapy, 20-30\% of esophageal cancer patients have been reported to have a pathologic complete response. ${ }^{1} \mathrm{~A}$ pathologic complete response represents the absence of viable tumor cells in the resection specimen after esophagectomy, and yet, these patients were subjected to a highly complex and invasive surgical procedure. These patients may benefit more from an active surveillance policy, in which frequent diagnostic evaluations after completion of neoadjuvant chemoradiotherapy may facilitate safe implementation of an organ-sparing approach. Conversely, patients with a poor response to neoadjuvant chemoradiotherapy (18\%) are likely to benefit less or not at all from neoadjuvant chemoradiotherapy, but are exposed to its side-effects. ${ }^{1}$ In these patients, ineffective therapy might be stopped and/or alternative treatment strategies (e.g. additional neoadjuvant treatment or upfront surgery) might be explored.

An interesting framework towards a more individualized approach for patients with an assumed pathologic complete response after chemoradiotherapy has been suggested earlier. ${ }^{29}$ Roughly 3 main questions are to be answered before an individualized approach might become reality. First, what is the accuracy is of clinical restaging, and which diagnostic modalities should be used? Second, what is the risk of surgery for this patient based on his preexistent comorbidities, functional status after neoadjuvant chemoradiotherapy and objective evaluation of a preoperative workup (e.g. pulmonary function testing)? And third, what is the risk of surveillance for this patient, including the consequences of missed 
residual cancer and salvage surgery? ${ }^{29}$ And above all, how accurately can all these risks be predicted for an individual, and how should these risks and benefits be weighed?

A high level of confidence in predicting pathologic complete response is a must for implementing an esophageal preservation strategy, but accurate treatment response assessment is a very complex endeavor. Several diagnostic modalities - including diffusionweighted magnetic resonance imaging (DW-MRI) and ${ }^{18} \mathrm{~F}$-fluorodeoxyglucose positron emission tomography and computed tomography $\left({ }^{18} \mathrm{~F}-\mathrm{FDG}\right.$ PET-CT $)$ as discussed in this thesis - have shown potential for response evaluation, but do not yet provide satisfactory results to justify clinical decision-making. Important remarks when reflecting upon the results of the studies on treatment response assessment as presented in this thesis and its corresponding possibilities, are amongst others the (technical) challenges in the use of advanced imaging techniques, heterogeneity in outcome evaluation and the risks of disease recurrence in pathologic complete responders.

\section{Opportunities for image-guided response assessment}

Traditionally, imaging end points have been limited to unidirectional size measurements, as per Response Evaluation Criteria in Solid Tumors (RECIST) criteria. ${ }^{30}$ However, biomarkers that inform on changes in tumor cellular architecture and functional characteristics are increasingly being explored. ${ }^{30} \mathrm{DW}-\mathrm{MRI}$ is a functional imaging modality that allows for tissue characterization by deriving image contrast from restriction in the free diffusion (i.e. random mobility or Brownian motion) of water molecules, which is related to microstructural tissue organization. An apparent diffusion coefficient (ADC) map can be derived from the DW-MRI images to quantify the diffusion restriction in a certain volume of interest. The ADC is inversely correlated with tissue cellularity. As chemoradiotherapy can result in the loss of cell membrane integrity, tumor response can be detected as an increase in tumor ADC. The studies presented in this thesis (chapter 5 and 6) rely on relative differences in $\mathrm{ADC}$ values as predictors for response to during neoadjuvant chemoradiotherapy.

The second modality under research in this thesis is ${ }^{18} \mathrm{~F}$-FDG PET-CT, which depends on the increased accumulation of ${ }^{18} \mathrm{~F}$-FDG within tumorous tissue relative to normal tissue. ${ }^{31}$ Changes in the accumulation of ${ }^{18} \mathrm{~F}-\mathrm{FDG}$ have been shown to be useful as an imaging biomarker for assessing response to treatment, for which the standardized uptake value (SUV) is frequently applied as a measurement of relative tissue/organ uptake. ${ }^{31}$ This measure compensates for the amount of injected FDG and the patient size, and as such facilitates comparisons between patients. ${ }^{31}$ The results of DW-MRI and ${ }^{18} \mathrm{~F}-\mathrm{FDG}$ PET-CT for treatment response assessment presented in this thesis are promising, but several opportunities for improvement remain in image-based assessment of pathologic response to treatment.

First, whole-tumor summary statistics (such as mean ADC or SUV values) are easily 
applicable, but fail to fully address the important issue of tumor heterogeneity. ${ }^{32}$ Voxel-based analytical methods, where changes in individual voxels can be monitored, may provide more precise results for response assessment. ${ }^{33}$

Second, cardiac triggering and respiratory gating techniques have been introduced to improve image quality by reducing motion artifacts. ${ }^{34}$ Even when applying these techniques, insufficient scan quality led to the exclusion of up to $9 \%$ of patients from analyses presented in this thesis due to inadequate tumor visibility. As such, improved motion management techniques in future studies may facilitate substantial improvements in pathologic complete response prediction.

Third, ADC values are frequently calculated based on a mono-exponential fit on $3 \mathrm{~b}$-values (diffusion weights). However, as signal decay as a function of b-values is not a monoexponential process and depends on perfusion in the lower range of $b$-values ${ }^{35}$, intravoxel incoherent motion (IVIM) as a method by which the diffusion component can be separated from the perfusion component may allow for a more precise assessment of the tumor and might contribute to characterizing differentiation grades and response rates of esophageal cancer in the future. ${ }^{36}$

\section{Improve clinical applicability of image-guided response assessment}

So far, the use of the aforementioned quantitative image-guided methods for response assessment in esophageal cancer is quite time-consuming. Clinical applicability could be improved by minimizing the duration of scan processing, standardization of imaging protocols as well as automatic delineation of tumor volumes on the scans. A first step forward is the use of semi-automated volumetric delineation methods (applied in chapters 5 and 6), of which the interobserver reproducibility of tumor delineations on DW-MRI and ADC measurements has shown to be very good. ${ }^{33,37}$

Clinical applicability could also be improved by a visual radiological assessment for residual disease on (DW-)MRI and ${ }^{18} \mathrm{~F}$-FDG PET-CT scans after neoadjuvant chemoradiotherapy. A pilot study including 51 esophageal cancer patients has pursued such an approach for MRI, resulting in high sensitivity (90-97\%) - reflecting a low chance of missing residual disease after neoadjuvant chemoradiotherapy -, but low specificity (42-50\%). This results in overstaging of complete responders as having residual tumor and, consequently, overtreatment. ${ }^{38}$ Also, the interobserver agreement between 3 readers was only $0.55-0.71 .{ }^{38}$ As such, this method is not yet ready for routine use in clinical practice but standardized guidelines on the image-based assessment of residual disease could improve these results in the future.

Ideally, a fully automatic tumor contouring tool (which is currently explored by the MD Anderson Cancer Center) would facilitate fast extraction of important features without human intervention and thus eliminate interobserver variation. In combination with the 
emerging field of radiomics - the extraction of a large amount of features from radiographic medical images using data-characterization algorithms - and the possibility of sharing large imaging datasets, this field of research has the potential to uncover disease characteristics that fail to be appreciated by the naked eye. Ultimately, the aim is to develop a setting in which such an automatic contouring and feature extraction tool is an integrated element of radiology programs radiologists use to evaluate clinical progress during a multidisciplinary tumor board meeting. This element should automatically contour the tumor volume, deliver corresponding ADC, SUV and radiomic values and use these values for a prediction model (such as presented in chapter 5 and 6, and currently under development by the study described in chapter 7). This model should translate these features into a predicted probability of a pathologic complete response or poor response based on those clinical and imaging characteristics, which should subsequently be discussed with the patient in order to make treatment decisions.

\section{Alternatives to image-guided response assessment}

Next to ${ }^{18} \mathrm{~F}$-FDG PET-CT and DW-MRI, endoscopic assessments with (bite-on-bite) biopsies after neoadjuvant chemoradiotherapy are frequently proposed to rule out potential residual cancer cells. Obviously, physicians would not want to withhold surgery in patients who have residual tumor cells based on biopsies after neoadjuvant chemoradiotherapy. For patients with negative biopsies however, the consequences are not so straightforward. As the distribution of residual cancer cells after chemoradiotherapy is unpredictable, and can be in the mucosal membrane, adventitia area or even in the lymph nodes, it is difficult to clinically detect residual chemoradiotherapy resistant tumors. ${ }^{39,40}$ In a more recent study using bite-on-bite biopsies after neoadjuvant chemoradiotherapy, the proportion falsenegative biopsies for the detection of TRG 3-4 tumors is still 10\% (with a 95\% confidence interval ranging from $4 \%$ to $23 \%) .{ }^{41}$

Additionally, circulating tumor DNA (ctDNA) - reflecting the presence and systemic burden of cancer - has emerged as a new valuable biomarker in the prediction of patient prognosis. $^{42-46}$

In conclusion, the most useful studies are probably those that examine the combined ability of multiple restaging modalities. This approach is more pragmatic and provides the closest approximation of a provider's actual clinical approach to decision making. The Dutch PRIDE study (chapter 7) aims at determining the accuracy of various diagnostic modalities for pathologic complete response assessment in a large cohort of 200 patients and incorporates quantitative IVIM analyses and visual assessment of high-resolution DW-MRI with endoscopic assessments and ctDNA levels. Furthermore, 2 other large European trials (recruiting $\geq 200$ patients) are currently studying the safety of active surveillance after a complete clinical response - as assessed with varying diagnostic modalities (the 
Table 2. Summary of suggestions for improvements in treatment response assessment in esophageal cancer.

\begin{tabular}{ll}
\hline Cluster & Suggestions for improvements \\
\hline General image-guided response assessment & $\begin{array}{l}\text { Voxel-based analytical methods } \\
\text { Full automatic delineation of tumor volume to } \\
\text { increase reproducibility and reduce duration of } \\
\text { assessment }\end{array}$ \\
\hline MRI-based response assessment & $\begin{array}{l}\text { Improve scan quality, e.g. the development of motion } \\
\text { management techniques other than cardiac triggering } \\
\text { and respiratory gating }\end{array}$ \\
& Bi-exponential model for ADC calculation (IVIM) \\
\hline $\begin{array}{l}\text { Alternatives to image-guided treatment response } \\
\text { assessment }\end{array}$ & $\begin{array}{l}\text { Endoscopic assessment of residual tumor with } \\
\text { bite-on-bite biopsies }\end{array}$ \\
& Liquid biopsies (e.g. circulating tumor DNA) \\
\hline
\end{tabular}

French ESOSTRATE trial [NCT02551458] and the Dutch SANO trial [Netherlands Trial Register ID NTR6803]). The results of these 3 trials together will hopefully be practice changing, and lead to a patient-tailored wait-and-see approach with omission of surgery or upfront surgery in the appropriate patients.

\section{Outcome evaluation}

The success of treatment response assessment does not only depend on the diagnostic modalities under research, but also on the histopathologic assessment of complete response as an outcome variable. The success of pathologic tumor regression as an outcome variable depends on inter- and intraobserver variability, as well as standardization of regression grading systems. For pathologists, it is a difficult endeavor to determine whether residual tumor cells are actually viable tumor cells which might lead to disease recurrence ${ }^{47}$, resulting in agreement varying from $0.44^{48}$ to $0.73^{49}$ for the Mandard tumor regression grading system in esophageal cancer. As there are many different grading systems of tumor regression after neoadjuvant therapy, experts call for a standardized system that homogenizes the application of tumor regression grading in gastrointestinal cancers. ${ }^{47}$ It has to be a simple and reproducible system, for which a 4 -tiered grading with the determination of the percentage of residual tumor was proposed. ${ }^{47}$ For the correct assessment of pathologic response to treatment, it is furthermore very important that the entire tumor bed is embedded for histopathological analysis, as small residual tumor infiltrates in the periphery otherwise won't be detected..$^{50}$ This may lead to better comparability of studies focusing on surgery and medical oncology that include pathologic tumor regression as relevant parameter of treatment effects and outcome. ${ }^{51}$

Second, not only the degree of tumor regression in primary tumor is important, but also 
tumor regression of tumor positive lymph nodes as these occur in up to $22 \%$ of patients with a pathologic complete response of the primary tumor after neoadjuvant chemoradiotherapy. ${ }^{52-57}$ These patients have a decreased survival compared to patients without positive lymph nodes as a consequence. ${ }^{52-57}$ Interestingly, the majority of these positive lymph nodes is situated within the neoadjuvant radiation field, possibly indicating a difference in radiosensitivity between the primary tumor and nodal metastases, but also between individual lymph nodes. ${ }^{53}$ The differentiation between benign and malignant lymph nodes on MRI remains challenging as non冈enlarged nodes may harbor malignant cells and the field of view of the MRI scans is focused on the primary tumor instead of complete peri-esophageal tissue. Other response assessment tools, such as endoscopic ultrasonography with lymph node biopsies or ${ }^{18} \mathrm{~F}-\mathrm{FDG}$ PET-CT, should thus be added for the detection of lymph node metastases after neoadjuvant chemoradiotherapy.

Last, pathologic response is not 1-to-1 related with survival and - as stated before - survival is not the only patient-relevant outcome in oncologic research. ${ }^{47} \mathrm{~A}$ clinical response to treatment might well be observed, but at the expense of worsening the patient's condition. HRQOL might even outweigh survival when deciding on treatment options ${ }^{58}$, which led to the widespread incorporation of HRQOL and other patient-reported outcomes as an important endpoint when evaluating treatment efficacy. ${ }^{59}$ This has already been executed by for example the previously mentioned ESOPEC trial, which compares nCRT versus preoperative chemotherapy for esophageal cancer.

\section{Optimal care for clinical complete responders}

As mentioned before, the risk of surveillance, including the consequences of missed residual cancer and salvage surgery, is an important factor when defining an individualized approach for patients with an assumed pathological complete response after chemoradiotherapy. ${ }^{29} \mathrm{It}$ is important to recognize that $20-40 \%$ of pathologic complete responders develop cancer recurrence within the 24 months after the completion of the trimodality strategy, irrespective of the histological subtype. ${ }^{60-63}$ These patients might be eligible for salvage surgery, but the increased frequency and severity of postoperative complications is an emerging issue after salvage surgery. ${ }^{40}$ There is also some evidence that salvage surgery is associated with a high proportion of tumor progression and worsened overall survival. ${ }^{40}$ As such, patients and multidisciplinary tumor boards should be made aware of these differences in outcomes and salvage should be reserved for practice in high-volume institutions. ${ }^{64}$

Contrarily, some believe that a small amount of residual disease $(<10 \%$, TRG 2$)$ can initially be missed in clinical response assessment, as long as this residual disease is detected during active surveillance, while the tumor is still resectable. ${ }^{65}$ The percentage of disease recurrence is however even higher in patients with a TRG2 than the already substantial number of $20-40 \%$ disease recurrence in pathologic complete responders, which questions whether 
Table 3. Pitfalls and suggestions for improvement in the use of histopathologic treatment response assessment to neoadjuvant chemoradiotherapy as outcome variable for treatment efficacy in esophageal cancer

\begin{tabular}{ll}
\hline Pitfalls & Suggestion for improvement \\
\hline $\begin{array}{l}\text { Inter- and intraobserver variability between } \\
\text { pathologists }\end{array}$ & $\begin{array}{l}\text { Consistent use of a 4-tiered simple and reproducible } \\
\text { grading system }\end{array}$ \\
Lack of standardization of pathologic response rates & $\begin{array}{l}\text { Include lymph node status (pN status) using other } \\
\text { response assessment tools (such as EUS with biopsies } \\
\text { or }{ }^{18} \mathrm{~F}-\mathrm{FDG} \text { PET-CT) }\end{array}$ \\
\hline Single focus on primary tumor (pT status) & $\begin{array}{l}\text { Include survival as outcome variable in trials } \\
\text { evaluating treatment response assessment }\end{array}$ \\
\hline Pathologic response is not 1-to-1 related with survival
\end{tabular}

this is a valid assumption. This especially holds when during this time interval distant metastases develop. Also, the selection of a patient for active surveillance - and corresponding cost-intensive follow-up - who turns out to need delayed surgery because of disease recurrence would be the least beneficial from an economical perspective. As such, the cost-effectiveness of organ-sparing treatment is to be determined.

Several studies have been initiated comparing active surveillance versus upfront surgery after chemoradiotherapy (the French ESOSTRATE trial, the Korean ESOPRESSO trial and the Dutch SANO trial), of which the ESOPRESSO trial is so far the only published randomized trial including 86 patients. It was concluded that close observation with salvage surgery might be a reasonable option in terms of overall survival in patients with resectable esophageal squamous cell carcinoma achieving clinical complete response after chemoradiotherapy ${ }^{66}$ However, the patients included in this trial all had squamous cell carcinoma and treatment consisted of 2 cycles of induction chemotherapy and then chemoradiotherapy of 50.4 Gy in 28 fractions, which is not generalizable to the Western world. A retrospective propensity score matched study conducted in The Netherlands concluded that overall survival and progression free survival in patients with clinical complete response undergoing active surveillance or immediate surgery were not significantly different. ${ }^{67}$ However, it is very important to realize that only 29 patients could be matched between both groups in this study, strongly questioning the validity of this conclusion for the entire esophageal cancer population.

Next to disease specific consequences of surveillance strategies, patients' preferences regarding active surveillance or standard esophagectomy when no residual cancer is observed after completion of neoadjuvant chemoradiotherapy should be taken into account when determining the optimal treatment strategy. A discrete choice experiment learned 
us that substantial heterogeneity is observed in patients' preferences for either active surveillance or standard esophagectomy after a clinical complete response. ${ }^{68}$ Also, for high risk surgical patients with significant comorbidities, a lower accuracy of a predicted pathological complete response might be deemed sufficient to postpone standard esophagectomy, whereas for younger, low risk surgical patients, a higher accuracy might be desired.

\section{Risk-stratified treatment}

Ultimately, we should aim for a risk-stratified selection of therapy based on local tumor response and the burden of distant disease in esophageal cancer patients. In this proposed risk stratified strategy, ctDNA levels might be determined in all patients with locally advanced esophageal cancer without apparent distant metastases upon clinical staging. Patients with high ctDNA levels - representing a high burden of disease - might be offered induction chemotherapy tailored to either squamous cell carcinomas or adenocarcinomas, and patients with a good response might subsequently proceed to chemoradiotherapy. Patients with a poor response might need to switch their chemotherapy regimen, or could be included in novel trials, but should not proceed to the standard treatment pathway with chemoradiotherapy followed by esophagectomy.

Patients with low or undetectable ctDNA levels before treatment might be offered chemoradiotherapy directly. Following chemoradiotherapy, locoregional tumor response can be determined based on changes in MRI parameters $(\triangle \mathrm{ADC}(\%))$ half-way during chemoradiotherapy, ${ }^{18} \mathrm{~F}-\mathrm{FDG}$ PET-CT, endoscopic biopsy and ctDNA levels. This would lead to a further stratification between patients: patients with a good response on MRI and undetectable ctDNA levels might be locoregionally cured by chemoradiotherapy alone and might be offered an active surveillance strategy instead of surgery, potentially increasing quality of life with this organ-preserving strategy. Patients with a poor response on imaging but undetectable ctDNA levels after chemoradiotherapy will be offered surgical resection, as these probably still have residual locoregional disease. Lastly, patients with high ctDNA levels after chemoradiotherapy, irrespective of their response on imaging, might be offered chemotherapy, as high ctDNA levels might reflect occult metastatic disease and these patients thus might not benefit from surgical resection.

In summary, the need for individualized treatment based on response assessment to neoadjuvant chemoradiotherapy for esophageal cancer is clear. Several challenges in response assessment have been discussed and active surveillance strategies have been proposed. Both topics should be assessed in detail within trials the coming years to demonstrate whether a safe organ-sparing treatment strategy could become reality for a select group of patients with esophageal cancer. 


\section{Surgical treatment}

With the implementation and favorable results of neoadjuvant therapy, curation is possible for an increased number of patients with esophageal cancer. Technical advances such as robot-assisted minimally invasive esophagectomy demonstrated to reduce the relatively high morbidity associated with esophagectomy, while enabling a safe and radical oncologic resection..$^{69}$ However, adequate staging and thorough examination of the general condition remain essential to select patients who are eligible for and would benefit from esophagectomy.

Accurate patient selection for surgery depends on tumor characteristics (such as TNM stage and tumor location), but also on assessment of comorbidities, patient nutritional status, and cardiopulmonary function. Recognition of the increased risk for not proceeding to surgery after starting neoadjuvant chemoradiotherapy or developing postoperative complications could aid the complex decision-making process of surgical treatment of patients with esophageal cancer. If this patient group can be selected prior to treatment, patient-individualized treatment (balancing the benefits and risks of esophagectomy for a specific patients), centralization of oncological care and counseling might result in a larger proportion of patients who will undergo successful esophagectomy.

The time between diagnosis and neoadjuvant chemoradiotherapy, as well as the interval between neoadjuvant chemoradiotherapy and esophagectomy, is highly suitable for interventions to optimize patients before surgery. Patients at high risk for not proceeding to surgery or postoperative complications could be more fully optimized before surgery by means of preventive measures, such as physical therapy to increase cardiorespiratory function and improvement of nutritional status. ${ }^{70-72}$ The use of real world evidence in chapter 10 learned that age, BMI and performance status are risk factors for not proceeding to surgery after starting neoadjuvant chemoradiotherapy. Even though age as risk factors is not modifiable, the results could aid physicians in the identification of patients who might benefit from increased counseling in the preoperative trajectory. These patients could be offered supervised prehabilitation for surgery, a preoperative geriatric assessment to obtain a holistic view of the patient or more frequent follow-up visits with their own oncologist or surgeon to motivate them and their caregivers for surgery.

Furthermore, stratification of patients by risk of postoperative complications for intervention trials may improve the effects of the interventions under investigation - such as Enhanced Recovery After Surgery (ERAS) programs ${ }^{73}$ or ischemic conditioning to increase perfusion of the gastric tube ${ }^{74}$. As an example, the identification of generalized arterial calcification as a risk factor for anastomotic leakage in chapter 11 forms the basis of the recently initiated 
multicenter phase-II intervention trial evaluating the safety and feasibility of laparoscopic ischemic conditioning of the gastric tube prior to transthoracic esophagectomy in patients with major arterial calcifications (ISCON-trial, ClinicalTrials.gov Identifier NCT03896399).

Lastly, as neoadjuvant therapy can induce high degrees of tumor regression, current nonsurgical patients (due to advanced local tumor characteristics) may become new surgical candidates. ${ }^{75}$ It has been reported feasible and beneficial in terms of survival to perform a radical resection combined with off-pump descending aorta replacement and adjuvant chemotherapy in 47 patients with a T4b esophageal tumor invading the aorta. ${ }^{76}$ Also, there is evidence that patients with cT4b tumors can be safely treated with robot-assisted minimally invasive esophagectomy after long-course chemoradiotherapy with a radical resection rate of $90 \% .^{75}$

\section{Final remarks}

The research presented in this thesis contributes to a more nuanced decision-making for esophageal cancer patients. Over the last years, personalized medicine has become a popular word in medical (cancer) research. We aim to differentiate treatment strategies per patient, but will inevitably be limited by studies that analyze outcomes on a group level - even though we are provided with more detailed patient and tumor genomic information using big data. The risk stratified selection of treatment as described before in this chapter is one example of an approach to research outcomes of specific treatments in smaller subgroups of patients. But above all, we - as physicians - should remember that the incorporation of the preferences of our patients in treatment decision making is the only way to truly deliver personalized medicine. 


\section{Conclusions}

\section{Part I. Neoadjuvant chemoradiotherapy}

- Physical functioning deteriorates and fatigue increases in esophageal cancer patients who underwent neoadjuvant chemoradiotherapy. The occurrence of adverse events during neoadjuvant chemoradiotherapy is associated with a sharper impairment of health-related quality of life and esophageal cancer-specific symptoms (chapter 2).

- Esophageal tumor motion during radiation treatment is highly variable between and within patients with esophageal cancer (chapter 3).

- A higher total neoadjuvant radiation dose increases pathologic response in terms of pathologic major response, but does not impact pathologic complete response rates in esophageal cancer patients (chapter 4).

\section{Part II. Treatment response assessment}

- Changes on ${ }^{18} \mathrm{~F}$-FDG PET/CT after neoadjuvant chemoradiotherapy and early changes on DW-MRI during neoadjuvant chemoradiotherapy can help identify pathologic complete response to neoadjuvant chemoradiotherapy in esophageal cancer and might be of complementary value in the assessment of pathologic complete response (chapter 5).

- The relative change in tumor cellularity as measured on DW-MRI during the first 2 weeks of neoadjuvant chemoradiotherapy is the most predictive for pathologic complete response in esophageal cancer patients compared to the other weeks of neoadjuvant chemoradiotherapy (chapter 6).

- A multi-center observational trial will develop a model that predicts the probability of pathologic complete response to neoadjuvant chemoradiotherapy in esophageal cancer, based on DW-MRI, DCE-MRI and ${ }^{18}$ F-FDG PET/CT (PRIDE study, chapter 7).

- Repeated imaging with both MRI and ${ }^{18} \mathrm{~F}-\mathrm{FDG}$ PET/CT is generally well tolerated by patients for the assessment of response to neoadjuvant chemoradiotherapy for esophageal cancer (chapter 8).

\section{Part III. Surgical treatment}

- Surgical treatment of esophageal cancer in the era of multimodality management focuses on the selection of patients who will benefit from surgery, based on their physical status and response to neoadjuvant therapy, as well as optimization of the entire perioperative trajectory (chapter 9).

- One in 7 patients who started neoadjuvant chemoradiotherapy for esophageal cancer do not proceed to surgical resection and have a decreased one-year overall survival compared to patients who do proceed to surgical resection (chapter 10). 
- Calcification of the coronary and supra-aortic arteries as measured on preoperative diagnostic CT scans are predictive of cervical anastomotic leakage after esophagectomy, suggesting that generalized cardiovascular disease is a strong indicator for the risk of leakage (chapter 11).

- Acute surgical interventions for esophageal and gastric cancer are rare. Minimally invasive surgery for gastric cancer is safe and feasible in the acute setting, with at least comparable postoperative clinical and short-term oncological outcomes compared to open surgery (chapter 12). 


\section{References}

1. van Hagen P, Hulshof MCCM, van Lanschot JJB, et al. Preoperative Chemoradiotherapy for Esophageal or Junctional Cancer. N Engl J Med. 2012;366(22):2074-2084.

2. Allum WH, Stenning SP, Bancewicz J, Clark PI, Langley RE. Long-term results of a randomized trial of surgery with or without preoperative chemotherapy in esophageal cancer. J Clin Oncol. 2009;27(30):50625067.

3. Cunningham D, Allum WWH, Stenning SSP, et al. Perioperative chemotherapy versus surgery alone for resectable gastroesophageal cancer. $N$ Engl J Med. 2006;355(1):11-20.

4. Ychou M, Boige V, Pignon J, et al. Perioperative chemotherapy compared with surgery alone for resectable gastroesophageal adenocarcinoma: an FNCLCC and FFCD multicenter phase III trial. J Clin Oncol. 2011;29(13):1715.

5. Sjoquist KM, Burmeister BH, Smithers BM, et al. Survival after neoadjuvant chemotherapy or chemoradiotherapy for resectable oesophageal carcinoma: an updated meta-analysis. Lancet Oncol. 2011;12(7):681-692.

6. Lagergren J, Smyth E, Cunningham D, Lagergren P. Oesophageal cancer. Lancet. 2017;390(10110):23832396.

7. Noordman BJ, E Verdam MG, Lagarde SM, et al. Impact of neoadjuvant chemoradiotherapy on healthrelated quality of life in long-term survivors of esophageal or junctional cancer: results from the randomized CROSS trial. Ann Oncol. 2018;29:445-451.

8. Noordman BJ, Verdam MGE, Lagarde SM, et al. Effect of Neoadjuvant Chemoradiotherapy on HealthRelated Quality of Life in Esophageal or Junctional Cancer: Results From the Randomized CROSS Trial. J Clin Oncol. 2018;36(3):268-275.

9. Blazeby JM, Sanford E, Falk SJ, Alderson D, Donovan JL. Health-related quality of life during neoadjuvant treatment and surgery for localized esophageal carcinoma. Cancer. 2005;103(9):1791-1799.

10. Xu C, Guo L, Liao Z, et al. Heart and lung doses are independent predictors of overall survival in esophageal cancer after chemoradiotherapy. Clin Transl Radiat Oncol. 2019;17:17-23.

11. Lagendijk JJW, Raaymakers BW, Raaijmakers AJE, et al. MRI/linac integration. Radiother Oncol. 2008;86(1):25-29.

12. Lips I, Lever F, Reerink O, et al. SU-E-J-57: MRI-Linac (MRL) Guided Treatment for Esophageal Cancer. Med Phys. 2012;39(6Part6):3665-3665.

13. Lin SH, Merrell KW, Shen J, et al. Multi-institutional analysis of radiation modality use and postoperative outcomes of neoadjuvant chemoradiation for esophageal cancer. Radiother Oncol. 2017;123(3):376-381.

14. Ono T, Wada H, Ishikawa H, Tamamura H, Tokumaru S. Clinical results of proton beam therapy for esophageal cancer: Multicenter retrospective study in japan. Cancers (Basel). 2019;11(7).

15. Yu W, Cai X-W, Liu Q, et al. Safety of dose escalation by simultaneous integrated boosting radiation dose within the primary tumor guided by (18)FDG-PET/CT for esophageal cancer. Radiother Oncol. 2015;114(2):195-200.

16. Welsh JW, Seyedin SN, Allen PK, et al. Local Control and Toxicity of a Simultaneous Integrated Boost for Dose Escalation in Locally Advanced Esophageal Cancer: Interim Results from a Prospective Phase I/II Trial. J Thorac Oncol. 2017;12(2):375-382.

17. Cheng N, Liu Y, Zhao G, et al. Phase I Trial of Intensity-Modulated Hyperfractionated Radiotherapy Boost with Concurrent Chemotherapy Immediately Following Standard Chemoradiotherapy in Advanced Esophageal Cancer. Int J Radiat Oncol Biol Phys. 2019.

18. Gaspar LE, Winter K, Kocha WI, Coia LR, Herskovic A, Graham M. A phase I/II study of external beam radiation, brachytherapy, and concurrent chemotherapy for patients with localized carcinoma of the esophagus (Radiation Therapy Oncology Group Study 9207): final report. Cancer. 2000;88(5):988-995.

19. Al-Batran S-E, Homann N, Pauligk C, et al. Perioperative chemotherapy with fluorouracil plus leucovorin, oxaliplatin, and docetaxel versus fluorouracil or capecitabine plus cisplatin and epirubicin for locally advanced, resectable gastric or gastro-oesophageal junction adenocarcinoma (FLOT4): a ra. Lancet. 2019;393(10184):1948-1957.

20. Reynolds J, Preston S, O’Neill B, et al. ICORG 10-14: NEOadjuvant trial in Adenocarcinoma of the oEsophagus and oesophagoGastric junction International Study (Neo-AEGIS). BMC Cancer. 2017;17(1):401. 
21. Hoeppner J, Lordick F, Brunner T, et al. ESOPEC: prospective randomized controlled multicenter phase III trial comparing perioperative chemotherapy (FLOT protocol) to neoadjuvant chemoradiation (CROSS protocol) in patients with adenocarcinoma of the esophagus (NCT02509286). BMC Cancer. 2016;16(1):503.

22. Tang H, Tan L, Shen Y, et al. CMISG1701: a multicenter prospective randomized phase III clinical trial comparing neoadjuvant chemoradiotherapy to neoadjuvant chemotherapy followed by minimally invasive esophagectomy in patients with locally advanced resectable esophageal squamous cell carcinoma (cT34aN0-1M0) (NCT03001596). BMC Cancer. 2017;17(1):450.

23. Mukherjee S, Hurt CN, Gwynne S, et al. NEOSCOPE: a randomised Phase II study of induction chemotherapy followed by either oxaliplatin/capecitabine or paclitaxel/carboplatin based chemoradiation as pre-operative regimen for resectable oesophageal adenocarcinoma. BMC Cancer. 2015;15(1):48.

24. Messager M, Mirabel X, Tresch E, et al. Preoperative chemoradiation with paclitaxel-carboplatin or with fluorouracil-oxaliplatin-folinic acid (FOLFOX) for resectable esophageal and junctional cancer: the PROTECT-1402, randomized phase 2 trial. BMC Cancer. 2016;16(1):318.

25. Kelly RJ. The emerging role of immunotherapy for esophageal cancer. Curr Opin Gastroenterol. 2019;35(4):337-343.

26. Pasquali S, Guang Yim Ã, Vohra RS, et al. Survival After Neoadjuvant and Adjuvant Treatments Compared to Surgery Alone for Resectable Esophageal Carcinoma A Network Meta-analysis. Ann Surg. 2017;265(3):481-491.

27. Burt BM, Groth SS, Sada YH, et al. Utility of Adjuvant Chemotherapy After Neoadjuvant Chemoradiation and Esophagectomy for Esophageal Cancer. Ann Surg. 2017;266(2):297-304.

28. Ni W, Chen J, Xiao Z, et al. Adjuvant radiotherapy for stage pN1M0 esophageal squamous cell carcinoma: Results from a Chinese two-center study. Thorac Cancer. May 2019:1759-7714.13088.

29. Semenkovich TR, Meyers BF. Surveillance versus esophagectomy in esophageal cancer patients with a clinical complete response after induction chemoradiation. Ann Transl Med. 2018;6(4).

30. deSouza NM. Diffusion-weighted MRI in Multicenter Trials of Breast Cancer: A Useful Measure of Tumor Response? Radiology. 2018;289(3):628-629.

31. Kinahan PE, Fletcher JW. Positron emission tomography-computed tomography standardized uptake values in clinical practice and assessing response to therapy. Semin Ultrasound, CT MRI. 2010;31(6):496-505.

32. Padhani AR, Liu G, Koh DM, et al. Diffusion-weighted magnetic resonance imaging as a cancer biomarker: consensus and recommendations. Neoplasia. 2009;11(2):102-125.

33. Kwee RM, Dik AK, Sosef MN, et al. Interobserver Reproducibility of Diffusion-Weighted MRI in Monitoring Tumor Response to Neoadjuvant Therapy in Esophageal Cancer. Coppola D, ed. PLoS One. 2014;9(4):e92211.

34. van Rossum PSN, van Hillegersberg R, Lever FM, et al. Imaging strategies in the management of oesophageal cancer: what's the role of MRI? Eur Radiol. 2013;23(7):1753-1765.

35. Le Bihan D, Breton E, Lallemand D, Aubin ML, Vignaud J, Laval-Jeantet M. Separation of diffusion and perfusion in intravoxel incoherent motion MR imaging. Radiology. 1988;168(2):497-505.

36. Zhu S, Wei Y, Gao F, et al. Esophageal carcinoma: Intravoxel incoherent motion diffusion-weighted MRI parameters and histopathological correlations. J Magn Reson Imaging. 2019;49(1):253-261.

37. Fang P, Musall BC, Son JB, et al. Multimodal Imaging of Pathologic Response to Chemoradiation in Esophageal Cancer. Int J Radiat Oncol Biol Phys. 2018;102(4):996-1001.

38. Vollenbrock SE, Voncken FEM, van Dieren JM, et al. Diagnostic performance of MRI for assessment of response to neoadjuvant chemoradiotherapy in oesophageal cancer. Br J Surg. February 2019.

39. Neishaboori N, Wadhwa R, Nogueras-González GM, et al. Distribution of Resistant Esophageal Adenocarcinoma in the Resected Specimens of Clinical Stage III Patients after Chemoradiation: Its Clinical Implications. Oncology. 2015;89(2):65-69.

40. Ajani JA, Bhutani MS, Swisher SG. Oesophageal preservation in locally advanced oesophageal cancer. Lancet Oncol. 2018;19(9):e430.

41. Noordman BJ, Spaander MCW, Valkema R, et al. Detection of residual disease after neoadjuvant chemoradiotherapy for oesophageal cancer (preSANO): a prospective multicentre, diagnostic cohort study. Lancet Oncol. 2018;19(7):965-974.

42. Sclafani F, Smyth E, Cunningham D, Chau I, Turner A, Watkins D. A pilot study assessing the incidence and clinical significance of circulating tumor cells in esophagogastric cancers. Clin Colorectal Cancer. 2014;13(2):94-99. 
43. Reeh M, Effenberger KE, Koenig AM, et al. Circulating Tumor Cells as a Biomarker for Preoperative Prognostic Staging in Patients With Esophageal Cancer. Ann Surg. 2015;261(6):1124-1130.

44. Gopalan V, Lam AK. Circulatory Tumor Cells in Esophageal Adenocarcinoma. Methods Mol Biol. 2018;1756:177-186.

45. Creemers A, Krausz S, Strijker M, et al. Clinical value of ctDNA in upper-GI cancers: A systematic review and meta-analysis. Biochim Biophys Acta. 2017;1868(2):394-403.

46. Azad TD, Chaudhuri AA, Fang P, et al. Circulating tumor DNA analysis for detection of minimal residual disease after chemoradiotherapy for localized esophageal cancer. Gastroenterology. 2019 [Epub ahead of print].

47. Langer R, Becker K. Tumor regression grading of gastrointestinal cancers after neoadjuvant therapy. Virchows Arch. 2018;472(2):175-186.

48. Mirza A, Naveed A, Hayes S, et al. Assessment of Histopathological Response in Gastric and GastroOesophageal Junction Adenocarcinoma following Neoadjuvant Chemotherapy: Which Scoring System to Use? ISRN Pathol. 2012;2012:1-8.

49. Karamitopoulou E, Thies S, Zlobec I, et al. Assessment of tumor regression of esophageal adenocarcinomas after neoadjuvant chemotherapy: comparison of 2 commonly used scoring approaches. Am J Surg Pathol. 2014;38(11):1551-1556.

50. Thies S, Langer R. Tumor Regression Grading of Gastrointestinal Carcinomas after Neoadjuvant Treatment. Front Oncol. 2013;3:262.

51. Langer R, Becker K. Reply to the letter "How to standardize the evaluation of tumor regression grading of gastrointestinal cancers after neoadjuvant therapy?” by Dr. Nasierowska-Guttmejer and Dr. Szawlowski, VIAR-D-18-00181. Virchows Arch. 2018;473(2):257-258.

52. Lv H-W, Li Y, Zhou M-H, Cheng J-W, Xing W-Q. Remnant lymph node metastases after neoadjuvant therapy and surgery in patients with pathologic T0 esophageal carcinoma impact on prognosis. Medicine (Baltimore). 2017;96(26):e7342.

53. Schurink B, Seesing MFJ, Goense L, et al. ypT0N+ status in oesophageal cancer patients: Location of residual metastatic lymph nodes with regards to the neoadjuvant radiation field. Eur J Surg Oncol. 2019;45(3):454459.

54. Cho HJ, Kim Y-H, Kim HR, et al. Oncologic Outcomes According to Remnant Lymph Node Metastases in Pathologic T0 (ypT0) Esophageal Squamous Cell Carcinoma Following Prospective Neoadjuvant Therapy and Surgery. Ann Surg Oncol. 2015;22(6):1851-1857.

55. Blackham AU, Yue B, Almhanna $\mathrm{K}$, et al. The prognostic value of residual nodal disease following neoadjuvant chemoradiation for esophageal cancer in patients with complete primary tumor response. $J$ Surg Oncol. 2015;112(6):597-602.

56. Kim MP, Correa AM, Lee J, et al. Pathologic T0N1 esophageal cancer after neoadjuvant therapy and surgery: an orphan status. Ann Thorac Surg. 2010;90(3):884-890; discussion 890-1.

57. Wang Q, Yu S, Xiao Z, et al. Residual lymph node status is an independent prognostic factor in esophageal squamous cell Carcinoma with pathologic T0 after preoperative radiotherapy. Radiat Oncol. 2015;10(1):142.

58. Thrumurthy SG, Morris JJA, Mughal MM, Ward JB. Discrete-choice preference comparison between patients and doctors for the surgical management of oesophagogastric cancer. Br J Surg. 2011;98(8):11241131.

59. Bottomley A, Reijneveld JC, Koller M, et al. Current state of quality of life and patient-reported outcomes research. Eur J Cancer. 2019;121:55-63.

60. Vallböhmer D, Hölscher AH, DeMeester S, et al. A Multicenter Study of Survival After Neoadjuvant Radiotherapy/Chemotherapy and Esophagectomy for ypT0N0M0R0 Esophageal Cancer. Ann Surg. 2010;252(5):744-749.

61. Meguid RA, Hooker CM, Taylor JT, et al. Recurrence after neoadjuvant chemoradiation and surgery for esophageal cancer: Does the pattern of recurrence differ for patients with complete response and those with partial or no response? J Thorac Cardiovasc Surg. 2009;138(6):1309-1317.

62. Jipping KM, Hulshoff JB, van Amerongen EA, Bright TI, Watson DI, Plukker JTM. Influence of tumor response and treatment schedule on the distribution of tumor recurrence in esophageal cancer patients treated with neoadjuvant chemoradiotherapy. J Surg Oncol. 2017;116(8):1096-1102.

63. Bouabdallah I, Thomas PA, D'Journo XB. Recurrence in complete responders after trimodality therapy in esophageal cancer. J Thorac Dis. 2019;11:S1304-S1306. 
64. Markar SR, Karthikesalingam A, Penna M, Low DE. Assessment of Short-Term Clinical Outcomes following Salvage Esophagectomy for the Treatment of Esophageal Malignancy: Systematic Review and Pooled Analysis. Ann Surg Oncol. 2014;21(3):922-931.

65. Noordman BJ, van Lanschot JJB, SANO study group. Oesophageal preservation in locally advanced oesophageal cancer - Authors' reply. Lancet Oncol. 2018;19(9):e431.

66. Park SR, Yoon DH, Kim JH, et al. A Randomized Phase III Trial on the Role of Esophagectomy in Complete Responders to Preoperative Chemoradiotherapy for Esophageal Squamous Cell Carcinoma (ESOPRESSO). Anticancer Res. 2019;39(9):5123-5133.

67. van der Wilk BJ, Noordman BJ, Neijenhuis LKA, et al. Active Surveillance Versus Immediate Surgery in Clinically Complete Responders After Neoadjuvant Chemoradiotherapy for Esophageal Cancer. Ann Surg. 2019 [Epub ahead of print].

68. Noordman BJ, de Bekker-Grob EW, Coene PPLO, et al. Patients' preferences for treatment after neoadjuvant chemoradiotherapy for oesophageal cancer. Br J Surg. 2018;105(12):1630-1638.

69. Van Der Sluis PC, Van Der Horst S, May AM, et al. Robot-assisted Minimally Invasive Thoracolaparoscopic Esophagectomy Versus Open Transthoracic Esophagectomy for Resectable Esophageal Cancer A Randomized Controlled Trial. Ann Surg. 2018.

70. Inoue J, Ono R, Makiura D, et al. Prevention of postoperative pulmonary complications through intensive preoperative respiratory rehabilitation in patients with esophageal cancer. Dis Esophagus. 2013;26(1):68-74.

71. van Adrichem EJ, Meulenbroek RL, Plukker JTM, Groen H, van Weert E. Comparison of two preoperative inspiratory muscle training programs to prevent pulmonary complications in patients undergoing esophagectomy: a randomized controlled pilot study. Ann Surg Oncol. 2014;21(7):2353-2360.

72. Bower MR, Martin RCG. Nutritional management during neoadjuvant therapy for esophageal cancer. $J$ Surg Oncol. 2009;100(1):82-87.

73. Markar SR, Karthikesalingam A, Low DE. Enhanced recovery pathways lead to an improvement in postoperative outcomes following esophagectomy: systematic review and pooled analysis. Dis Esophagus. 2015;28(5):468-475.

74. Kechagias A, van Rossum PSN, Ruurda JP, van Hillegersberg R. Ischemic Conditioning of the Stomach in the Prevention of Esophagogastric Anastomotic Leakage After Esophagectomy. Ann Thorac Surg. 2016;101(4):1614-1623.

75. van Hillegersberg R, Seesing MFJ, Brenkman HJF, Ruurda JP. Robot-assisted minimally invasive esophagectomy. Der Chir. 2017;88(S1):7-11.

76. Cong Z, Diao Q, Yi J, et al. Esophagectomy Combined With Aortic Segment Replacement for Esophageal Cancer Invading the Aorta. Ann Thorac Surg. 2014;97(2):460-466. 



\section{APPENDICES}

\section{Summary in Dutch (Nederlandse samenvatting) \\ Authors and affiliations}

Review committee

List of publications Acknowledgements (Dankwoord)

Curriculum Vitae 


\section{Summary in Dutch (Nederlandse samenvatting)}

\section{Inleiding}

Slokdarmkanker is wereldwijd de zevende meest voorkomende vorm van kanker. Jaarlijks worden er bijna 600.000 patiënten met slokdarmkanker gediagnosticeerd, waarvan 2.500 in Nederland. In totaal komt $60 \%$ van deze patiënten in aanmerking voor een in opzet curatieve behandeling, die tegenwoordig bestaat uit chemotherapie en bestraling (de zogeheten neoadjuvante chemoradiotherapie) gevolgd door een slokdarmresectie. Het doel van neoadjuvante chemoradiotherapie is het verkleinen van de tumor, waardoor de kansen op een radicale resectie en overleving worden vergroot. Door de toevoeging van neoadjuvante chemoradiotherapie aan de behandeling is de vijfjaarsoverleving van patiënten gestegen van 33\% naar 47\%. Echter, dit betekent dat nog steeds de helft van de patiënten met slokdarmkanker zonder uitzaaiingen naar andere organen is overleden vijf jaar na de behandeling. Dit komt onder andere doordat niet elk onderdeel van de behandeling bij iedere patiënt even effectief is, bovendien de behandeling gepaard gaat met het risico op complicaties en tevens slokdarmkanker regelmatig recidiveert.

Uiteindelijk is het doel om iedere patiënt de optimale behandeling te geven, waarbij een zorgvuldige afweging van de voor- en nadelen hiervan centraal staat. In dat kader valt de afgelopen jaren veelvuldig de term personalized medicine wanneer er gesproken wordt over behandelstrategieën tegen kanker. Dit idee staat momenteel nog ver van de hedendaagse klinische praktijk, met name doordat het individuele behandelingseffect nog onvoldoende voorspeld kan worden om patiënten een behandeling op maat aan te kunnen bieden. Het doel van het onderzoek beschreven in dit proefschrift is om de verschillende onderdelen van de behandeling van slokdarmkanker te optimaliseren. In deel 1 wordt de neoadjuvante chemoradiotherapie behandeld, in deel 2 het voorspellen van het effect van neoadjuvante chemoradiotherapie en in deel 3 de chirurgische behandeling.

\section{Neoadjuvante chemoradiotherapie}

Neoadjuvante chemoradiotherapie bestaat voor patiënten met slokdarmkanker in Nederland uit chemotherapie en bestraling gedurende vijf weken, waarna na zes tot tien weken een slokdarmresectie plaatsvindt. Deel 1 verschaft inzicht in de mogelijkheden om de effectiviteit van de behandeling te optimaliseren en bijwerkingen te minimaliseren.

Het effect van neoadjuvante chemoradiotherapie en complicaties daarvan op de kwaliteit van leven van patiënten met slokdarmkanker wordt beschreven in hoofdstuk 2. Patiënten werden gevraagd om voor en na de behandeling met neoadjuvante chemoradiotherapie een vragenlijst in te vullen over hun kwaliteit van leven. Neoadjuvante chemoradiotherapie blijkt een negatief effect te hebben op het fysiek functioneren en de vermoeidheid, terwijl patiënten aan de andere kant minder last krijgen van pijn bij het slikken. Patiënten die 
complicaties ondervonden van de behandeling hadden één maand na de behandeling meer pijn, verlies van smaak en meer problemen met eten, vergeleken met patiënten zonder complicaties. Bovendien blijkt angst een veel voorkomend symptoom bij patiënten met slokdarmkanker, zowel voor als na de behandeling. Deze bevindingen benadrukken het belang om patiënten te informeren over de gevolgen van de behandeling op de kwaliteit van leven. Ook de kwaliteit van leven - en niet alleen de overleving - dient dus als uitkomst te gebruikt te worden in verder onderzoek naar behandelingen van slokdarmkanker.

Eén van de mogelijkheden om de bijwerkingen van neoadjuvante chemoradiotherapie te verminderen is een zeer precieze bestraling van de tumor, waardoor omliggend gezond weefsel wordt gespaard. Hiervoor kan gebruik worden gemaakt van beeldgestuurde bestraling en gepersonaliseerde bestralingsmarges (Figuur 1). Hoofdstuk 3 beschrijft als een eerste stap de variatie tussen patiënten in de beweging van de slokdarmtumor tijdens de bestraling. In deze studie ondergingen patiënten die werden behandeld met neoadjuvante chemoradiotherapie zes MRI scans. Tijdens deze cine-MRI scan worden er 75 scans binnen 45 seconden gemaakt, waardoor de beweging van de slokdarm kan worden onderzocht. De beweging van de slokdarmtumor vindt voornamelijk plaats in de craniocaudale (verticale) richting, veroorzaakt door de ademhaling. Daarnaast zijn er grote verschillen in de beweging van de slokdarmtumor binnen één patiënt en tussen verschillende patiënten. Het is dus noodzakelijk om niet alleen voorafgaand aan de bestraling de beweging van de slokdarmtumor vast te stellen, maar ook gedurende het traject van de neoadjuvante chemoradiotherapie.
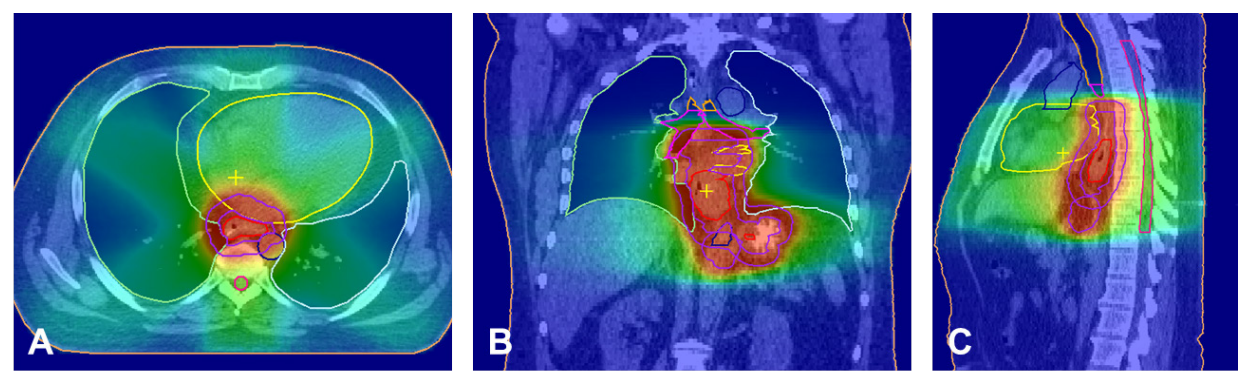

Figuur 1. Een voorbeeld van een bestralingsplan van een patiënt met slokdarmkanker waarin de dosis straling als een kleurenspectrum zichtbaar is. De slokdarmtumor is rood omlijnd en de onzekerheidsmarges voor de bestraling zijn paars omlijnd ( $A$ transversaal aanzicht, $B$ coronaal aanzicht, $C$ sagittaal aanzicht). 
Behalve gepersonaliseerde bestralingsmarges, maakt beeldgestuurde bestraling het ook mogelijk om de bestralingsdosis te laten variëren binnen verschillende delen van de tumor (dose painting). Hoofdstuk 4 beantwoordt de vraag of een behandeling met een hogere neoadjuvante bestralingsdosis resulteert in een beter effect van de behandeling op de slokdarmtumor. Behandelstrategieën uit Nederland, België en de Verenigde Staten zijn met elkaar vergeleken. Hieruit blijkt dat er geen sprake is van een overtuigende dosis-respons relatie voor slokdarmkanker.

\section{Het voorspellen van het behandelingseffect}

Het effect van neoadjuvante chemoradiotherapie is niet bij iedere patiënt hetzelfde. Er is sprake van een pathologisch complete respons wanneer het effect van de neoadjuvante chemoradiotherapie zo goed is, dat er geen kankercellen meer worden gevonden in het slokdarmpreparaat bij microscopisch onderzoek na de slokdarmresectie. Bij ongeveer $30 \%$ van de patiënten is dat het geval en deze patiënten hebben de beste prognose. Een nauwkeurige voorspelling van een pathologisch complete respons zou onderzoekers in staat stellen om de haalbaarheid en uitkomsten van een orgaansparende behandelstrategie - waarbij een slokdarmresectie achterwege gelaten wordt - te onderzoeken.

Er is ook een groep patiënten waarbij er vrijwel geen effect meetbaar is van de neoadjuvante chemoradiotherapie (non-respons), terwijl de behandeling wel gepaard gaat met het risico op bijwerkingen. Deze patiënten zouden onder andere baat kunnen hebben bij een nieuwe vorm van neoadjuvante therapie. Er bestaat dus voldoende reden om de keuze voor neoadjuvante chemoradiotherapie en/of de noodzaak voor een slokdarmresectie af te stemmen op de individuele patiënt.

Het voorspellen van het effect van neoadjuvante chemoradiotherapie met behulp van beeldvormende technieken zoals positron emission tomography/computed tomography (PET/CT) en diffusion weighted magnetic resonance imaging (DW-MRI) wordt besproken in deel 2. Beide technieken betreffen functionele beeldvormende technieken; dat wil zeggen dat ze niet alleen informatie verschaffen over de anatomie maar ook over de functie van het weefsel of het orgaan. PET/CT maakt gebruikt van radioactief gelabeld glucose die zich concentreert in weefsels met een verhoogd metabolisme, zoals kankercellen of een ontsteking (Figuur 2). DW-MRI berust op de beweeglijkheid van watermoleculen als een marker voor celdichtheid binnen een weefsel. Over het algemeen is de celdichtheid van een tumor groter dan van het omliggende weefsel en zal de celdichtheid afnemen als de behandeling met chemoradiotherapie effectief is en kankercellen afsterven (Figuur 3). 

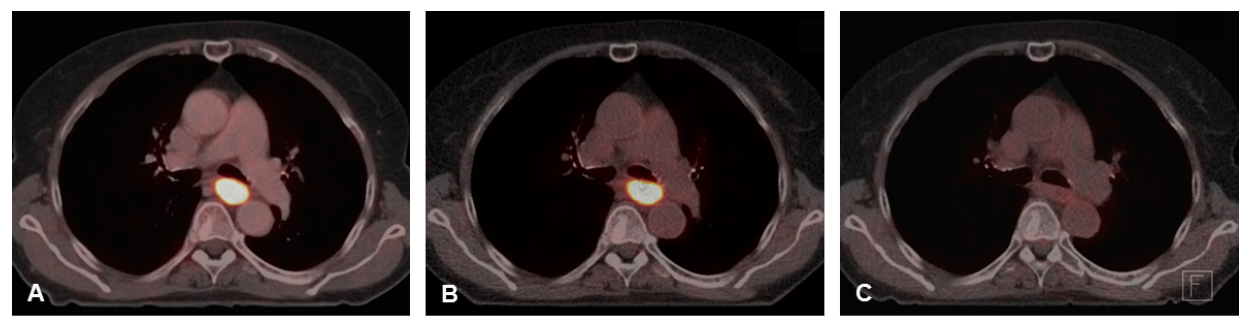

Figuur 2. Transversale weergaves van een PET/CT scan van een patiënt met slokdarmkanker voor $(A)$, tijdens $(B)$ en na $(C)$ de behandeling met neoadjuvante chemoradiotherapie. Er is een duidelijke afname zichtbaar van het signaal ter plaatse van de slokdarmtumor na de neoadjuvante chemoradiotherapie $(C)$.
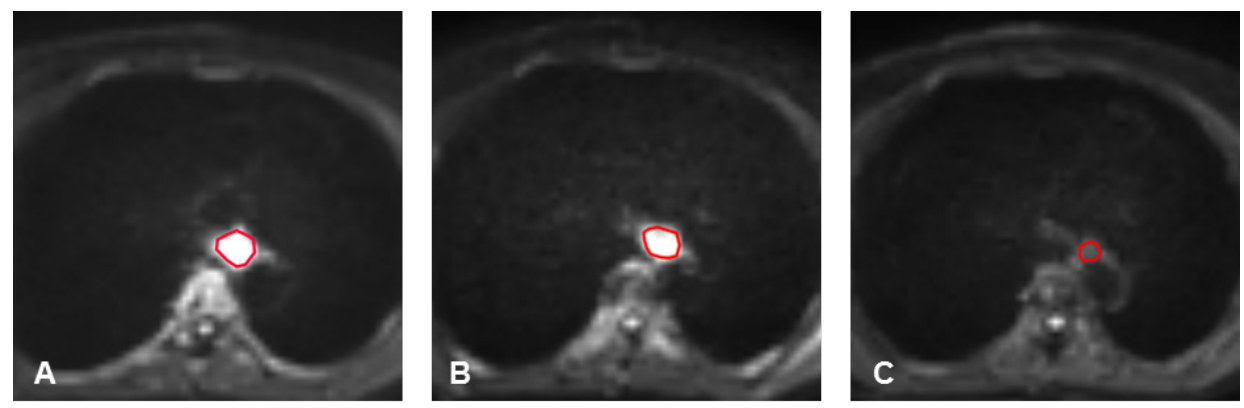

Figuur 3. Transversale weergaves van een DW-MRI scan van een patiënt met slokdarmkanker voor $(A)$, tijdens $(B)$ en na $(C)$ de behandeling met neoadjuvante chemoradiotherapie. Het versterkte signaal op de scan binnen de rode omlijning die de slokdarmtumor betreft wijst op een hoge celdichtheid en neemt af naarmate de behandeling vordert.

De afzonderlijke en gecombineerde waarde van PET/CT en DW-MRI voor het voorspellen van het effect van neoadjuvante chemoradiotherapie bij patiënten met slokdarmkanker wordt beschreven in hoofdstuk 5. Dit onderzoek is het resultaat van een samenwerking tussen het Universitair Medisch Centrum Utrecht, het Antoni van Leeuwenhoek Ziekenhuis in Amsterdam en het MD Anderson Cancer Center in Houston. Patiënten die deelnamen aan het onderzoek ondergingen een PET/CT scan en MRI scan voor, tijdens en na de neoadjuvante chemoradiotherapie. Veranderingen op de PET/CT scan na afloop van de neoadjuvante chemoradiotherapie bleken het meest nuttig voor het voorspellen van een pathologisch complete respons. Voor DW-MRI zijn dat de veranderingen op de scan tijdens de neoadjuvante chemoradiotherapie. Ook zijn PET/CT en DW-MRI mogelijk van toegevoegde waarde voor het voorspellen van een pathologisch complete respons. Met behulp van een model waarin de bevindingen van de PET/CT scans en DW-MRI scans werden gecombineerd, kon met $83 \%$ zekerheid worden vastgesteld welke patiënten een pathologisch complete respons hadden. 
Hoofdstuk 6 borduurt voort op bovenstaande bevindingen, namelijk door het optimale moment voor de DW-MRI scan te onderzoeken tijdens neoadjuvante chemoradiotherapie om het effect van de behandeling zo accuraat mogelijk te kunnen voorspellen. In deze prospectieve studie hebben patiënten tijdens de neoadjuvante chemoradiotherapie wekelijks een DW-MRI scan ondergaan. De verandering in signaal op DW-MRI scans gedurende de eerste twee weken van neoadjuvante chemoradiotherapie is uitermate veelbelovend voor het voorspellen van een pathologisch complete respons. Deze informatie wordt nu gebruikt om de belasting van patiënten te minimaliseren in de multicenter MRI validatiestudie, waarvan de achtergrond en het protocol worden beschreven in hoofdstuk 7. In deze studie wordt op basis van gegevens van PET/CT scans, MRI scans en circulerend tumor DNA (ctDNA) een model ontwikkelt waarmee het effect van neoadjuvante chemoradiotherapie op de tumor accuraat voorspeld kan worden. Dit model heeft als doel om in de toekomst slokdarmresecties veilig achterwege te laten bij patiënten met een hoge kans op een pathologisch complete respons na de behandeling met chemoradiotherapie. De zeer intensieve behandeling van slokdarmkanker zou daardoor beter afgestemd kunnen worden op de individuele patiënt met zijn of haar wensen.

Het tweede onderdeel van het proefschrift wordt afgesloten door hoofdstuk 8. Hierin wordt de fysieke en de mentale belasting van extra PET/CT scans en MRI scans gedurende de neoadjuvante chemoradiotherapie voor patiënten geëvalueerd met behulp van vragenlijsten. Het blijkt dat patiënten deze extra onderzoeken over het algemeen goed verdragen en dat zij weinig last hebben van pijn, angst, schaamte en ongemak. Patiënten geven MRI scans de voorkeur boven PET/CT scans. Zowel PET/CT en MRI scans worden vanuit het oogpunt van de patiënt goed verdragen en kunnen beide worden gebruikt voor respons evaluatie.

\section{Chirurgische behandeling}

De slokdarmresectie is een tot op heden essentieel onderdeel van de curatieve behandeling van patiënten met slokdarmkanker. Bij een slokdarmresectie wordt de continuïteit van het spijsverteringskanaal veelal hersteld door de maag door het middenrif richting de borstkas te mobiliseren en daar of in de hals te verbinden met het resterende deel van de slokdarm. Hierdoor wordt er een buismaag gecreëerd. Door de ligging van de slokdarm dichtbij het hart, de longen en de aorta is het een gecompliceerde procedure met een relatief hoog risico op complicaties en zelfs overlijden.

De onderzoeken beschreven in deel 3 van dit proefschrift zijn alle gericht op de rol van de chirurgische behandeling van slokdarmkanker in een tijd waarin verschillende behandelingen - zoals chemotherapie, radiotherapie en chirurgie - worden gecombineerd. 
In hoofdstuk 9 wordt de beschikbare literatuur over de rol van de chirurgische behandeling van slokdarmkanker uiteengezet. Uit deze review wordt duidelijk dat het van groot belang is om de juiste patiënten te selecteren voor een slokdarmresectie. Belangrijke factoren die hierbij een rol spelen, zijn de aanwezigheid van comorbiditeit, fysieke gesteldheid en de mate van respons op neoadjuvante therapie. Daarnaast wordt het perioperatieve traject voor patiënten de laatste jaren geïntensiveerd door de samenwerking met fysiotherapeuten, diëtisten en pijnspecialisten. Uiteraard hebben al deze ontwikkelingen als doel om de prognose van patiënten met slokdarmkanker te verbeteren.

Ondanks dat de voordelen van neoadjuvante chemoradiotherapie onomstotelijk zijn vastgesteld, is de behandeling geassocieerd met bijwerkingen. Dit is met name relevant wanneer deze bijwerkingen voorkomen dat de patiënt vervolgens een slokdarmresectie kan ondergaan. Door middel van een landelijke studie met data van de jaren 2015 en 2016, wordt in hoofdstuk 10 aangetoond dat maar liefst één op de zeven patiënten die starten met neoadjuvante chemoradiotherapie geen slokdarmresectie ondergaat. Deze patiënten hebben een slechtere overlevingskans dan patiënten die wel een slokdarmresectie ondergaan. Deze bevindingen kunnen als uitgangspunt fungeren voor toekomstig onderzoek, waarin onderzocht zou moeten worden of deze patiëntengroep kan worden geselecteerd voorafgaand aan de behandeling. Ook de centralisatie van oncologische zorg en een betere begeleiding zouden mogelijk kunnen leiden tot meer patiënten die een slokdarmresectie ondergaan.

Om postoperatieve complicaties met ernstige gevolgen te kunnen voorkomen of voortijdig te kunnen herkennen, is het belangrijk om inzicht te krijgen in risicofactoren voor het ontstaan van complicaties. Een gevreesde complicatie na een slokdarmresectie betreft lekkage van de verbinding tussen de buismaag en het resterende deel van de slokdarm (naadlekkage). Onze onderzoeksgroep heeft eerder onder andere aangetoond dat atherosclerose van de thoracale aorta geassocieerd is met het ontstaan van naadlekkage. Hoofdstuk 11 beschrijft of deze associatie gebaseerd is op problemen in de directe bloedvoorziening van de buismaag, of dat het een gegeneraliseerd vasculair probleem betreft. Aangetoond is dat calcificaties van de supra-aortale en coronaire arteriën voorspellend zijn voor naadlekkage na een slokdarmresectie. Hieruit kan worden geconcludeerd dat het inderdaad een gegeneraliseerd vasculair probleem betreft. Deze risicofactor kan worden meegenomen in prognostische modellen die individuele risico's op postoperatieve complicaties inschatten in de toekomst.

Tot slot is de toepassing van minimaal invasieve chirurgie een mogelijkheid om postoperatieve complicaties te verminderen. In de electieve setting wordt dit al veelvuldig 
toegepast voor slokdarmkanker. Echter, er is weinig bekend over de toepassing van minimaal invasieve chirurgie in de acute setting. Het beschreven onderzoek in hoofdstuk 12 toont met behulp van een landelijk cohort aan dat acute slokdarmresecties zeldzaam zijn. Daardoor konden complicaties van minimaal invasieve chirurgie en open chirurgie in de acute setting niet met elkaar worden vergeleken. Voor maagkanker daarentegen lijkt de toepassing van minimaal invasieve chirurgie in de acute setting veilig op het gebied van postoperatieve complicaties en oncologische resultaten.

\section{Conclusie}

Ondanks dat er alleen al in het afgelopen jaar meer dan 2.000 publicaties zijn verschenen over slokdarmkanker, is er nog veel onbekend op het gebied van de etiologie, prognose en behandeling van patiënten met slokdarmkanker. Om de prognose van slokdarmkankerpatiënten daadwerkelijk te kunnen verbeteren, is het van groot belang om onderzoek te doen waarvan de resultaten leiden tot veranderingen in de klinische praktijk. De onderzoeken beschreven in dit proefschrift dragen hieraan bij door de verschillende vormen van neoadjuvante chemoradiotherapie en de gevolgen hiervan te bestuderen, bovendien te streven naar accurate evaluatie van respons van de tumor op neoadjuvante chemoradiotherapie en door de chirurgische behandeling te optimaliseren. 


\section{Authors and affiliations}

\section{University Medical Center Utrecht, Utrecht, The Netherlands}

Department of Surgery

Lucas Goense, MD, PhD

Richard van Hillegersberg, MD, PhD

B. Feike Kingma, MD

Jelle P. Ruurda, MD, PhD

Department of Radiation Oncology

Mick R. Boekhoff, MSc

Ingmar L. Defize, MD

Sophie E. Heethuis, PhD

Jan J.W. Lagendijk, PhD

Astrid L.H.M.W. van Lier, $\mathrm{PhD}$

Gert J. Meijer, PhD

Stella Mook, MD, PhD

Peter S.N. van Rossum, MD, PhD

Department of Medical Oncology

Nadia Haj Mohammad, MD, PhD

Department of Radiology

Pim A. de Jong, MD, PhD

Helena M. Verkooijen, MD, PhD

Department of Nuclear Medicine

Marnix G.E.H. Lam, MD, PhD

Department of Pathology

Lodewijk A.A. Brosens, $\mathrm{MD}, \mathrm{PhD}$

Department of Gastroenterology

Bas L.A.M. Weusten, MD, PhD

The Netherlands Cancer Institute - Antoni van Leeuwenhoek Hospital

Department of Radiation Oncology

Berthe M.P. Aleman, MD, PhD

Francine E.M. Voncken, MD 
Department of Gastroenterology

Jolande M. van Dieren, MD, PhD

Department of Radiology

Annemariek Bartels-Rutten, MD, PhD

Leon C. ter Beek, PhD

Regina G.H. Beets-Tan, MD, PhD

Department of Surgery

Johanna W. van Sandick, MD, PhD

Amsterdam University Medical Centers, Academic Medical Center, Amsterdam, The Netherlands

Department of Radiation Oncology

Maarten C. Hulshof, MD, PhD

Department of Medical Oncology

Hanneke W.M. van Laarhoven, MD, PhD

Department of Gastroenterology

Jacques J. Bergman, $\mathrm{MD}, \mathrm{PhD}$

Department of Radiology and Nuclear Medicine

Roel J. Bennink, MD, PhD

Aart J. Nederveen, PhD

University Medical Center Groningen, Groningen, The Netherlands

Department of Radiation Oncology

Kristel (C.) T. Muijs, MD, PhD

Veronique E.M. Mul, MD

Department of Radiology

Hildebrand Dijkstra, PhD

Derya Yakar, MD, $\mathrm{PhD}$

Department of Gastroenterology and Hepatology

Wouter B. Nagengast, MD, PhD 
Department of Nuclear Medicine and Molecular Imaging

Walter Noordzij, MD

Department of Surgery

John T.M. Plukker, MD, PhD

Holland Proton Therapy Center, Delft, The Netherlands

Department of Radiation Oncology

Marco van Vulpen, MD, PhD

Radboud University Medical Center, Nijmegen, The Netherlands

Department of Radiation Oncology

Marcel Verheij, MD, PhD

Leiden University Medical Center, Leiden, The Netherlands

Department of Radiation Oncology

Irene M. Lips, MD, PhD

University Hospitals Leuven, Leuven, Belgium

Department of Radiation Oncology

Karin Haustermans, MD, PhD

Melissa Thomas, MD

Department of Surgery

Johnny Moons, RN, MScN

Philippe Nafteux, MD, PhD

Ghent University Hospital, Ghent, Belgium

Department of Radiation Oncology

Tom Boterberg, MD, $\mathrm{PhD}$

Department of Surgery

Elke van Daele, MD

Piet Pattyn, MD, PhD

Royal Military Academy, Brussels, Belgium

Department of Applied Mathematics

Christiaan Perneel, PhD 
The University of Texas MD Anderson Cancer Center, Houston, Texas, United States of America

Department of Radiation Oncology

Wei Deng, MD

Penny Fang, MD

Steven H. Lin, MD, PhD

Department of Imaging Physics

Jingfei Ma, PhD

Benjamin C. Musall, MSc

Moscow State University of Medicine and Dentistry/Moscow Clinical Scientific Center, Moscow, Russia

Department of Surgery

Sergey A. Domrachev, MD, PhD

Mikhail A. Koshkin, MD

Sao Paulo Institute of Cancer, University of Sao Paulo School of Medicine, Sao Paulo, Brasil

Department of Surgery

Flavio R. Takeda, MD, PhD 


\section{Review committee}

Prof. dr. P.A. de Jong

Professor of Radiology, University Medical Center Utrecht

Prof. dr. ir. J.J.W. Lagendijk

Professor of Radiation Oncology Physics, University Medical Center Utrecht

Prof. dr. M.R. Vriens

Professor of Surgery, University Medical Center Utrecht

Prof. dr. J.J.B. van Lanschot

Professor of Surgery, Erasmus Medical Center Rotterdam

Prof. dr. M. Verheij

Professor of Radiation Oncology, Radboud University Medical Center Nijmegen 


\section{List of publications}

1. Borggreve $\mathbf{A S}^{*}$, Goense $\mathrm{L}^{*}$, van Rossum PSN, et al. Preoperative prediction of pathologic response to neoadjuvant chemoradiotherapy in patients with esophageal cancer using 18F-FDG PET/CT and DW-MRI: a prospective multicenter study. Int J Radiat Oncol Biol Phys. 2020. [Epub ahead of print]

2. Borggreve $\mathbf{A S}^{\star}$, den Boer $\mathrm{RB}^{\star}$, van Boxel GI, et al. The Predictive Value of Low Muscle Mass as Measured on CT Scans for Postoperative Complications and Mortality in Gastric Cancer Patients: A Systematic Review and Meta-Analysis. J Clin Med. 2020;9(1):199.

3. Borggreve AS, Heethuis SE, Boekhoff MR, et al. Optimal timing for prediction of pathologic complete response to neoadjuvant chemoradiotherapy with diffusion-weighted MRI in patients with esophageal cancer. Eur Radiol. 2019. [Epub ahead of print]

4. Thomas $\mathbf{M}^{\star}$, Borggreve $\mathbf{A S}^{\star}$, van Rossum PSN, et al. Radiation dose and pathological response in oesophageal cancer patients treated with neoadjuvant chemoradiotherapy followed by surgery: a multi-institutional analysis. Acta Oncol. 2019;58(10):1358-1365.

5. Seesing MFJ, Borggreve AS, Ruurda JP, van Hillegersberg R. New-onset atrial fibrillation after esophagectomy for cancer. J Thorac Dis. 2019;11(Suppl 5):S831-S834.

6. Borggreve AS, van Rossum PSN, Mook S, et al. Frequency of surgical resection after starting neoadjuvant chemoradiotherapy in patients with esophageal cancer: A populationbased cohort study. Eur J Surg Oncol. 2019;45(10):1919-1925.

7. Borggreve AS, Goense L, Brenkman HJF, et al. Imaging strategies in the management of gastric cancer: current role and future potential of MRI. Br J Radiol. 2019;92(1097):20181044.

8. Borggreve AS, Mook S, Verheij M, et al. Preoperative image-guided identification of response to neoadjuvant chemoradiotherapy in esophageal cancer (PRIDE): a multicenter observational study.

BMC Cancer. 2018;18(1):1006.

9. Plate JDJ ${ }^{\star}$, Borggreve AS $^{\star}$, van Hillegersberg R, Peelen LM. Post Hoc Power Calculation: Observing the Expected. Ann Surg. 2019;269(1):e11. 
10. Heethuis SE, Borggreve AS, Goense L, et al. Quantification of variations in intrafraction motion of esophageal tumors over the course of neoadjuvant chemoradiotherapy based on cine-MRI. Phys Med Biol. 2018;63(14):145019.

11. Heethuis SE, Goense L, van Rossum PSN, Borggreve AS, et al. DW-MRI and DCE-MRI are of complementary value in predicting pathologic response to neoadjuvant chemoradiotherapy for esophageal cancer. Acta Oncol. 2018;57(9):1201-1208.

12. Borggreve $\mathbf{A S}^{\star}$, Kingma BF* ${ }^{\star}$ Domrachev SA, et al. Surgical treatment of esophageal cancer in the era of multimodality management. Ann N Y Acad Sci. 2018;1434(1):192-209.

13. Goense L, Meziani J, Borggreve AS, et al. Role of adjuvant chemoradiotherapy after endoscopic treatment of early-stage esophageal cancer: a systematic review. Minerva Chir. 2018;73(4):428-436.

14. Seesing MFJ, Scheijmans JCG, Borggreve AS, et al. The predictive value of new-onset atrial fibrillation on postoperative morbidity after esophagectomy. Dis Esophagus. 2018;31(11).

15. Goense L, Borggreve AS, Heethuis SE, et al. Patient perspectives on repeated MRI and PET/CT examinations during neoadjuvant treatment of esophageal cancer. Br J Radiol. 2018;91(1086):20170710.

16. Borggreve AS, Goense L, van Rossum PSN, et al. Generalized cardiovascular disease on a preoperative CT scan is predictive for anastomotic leakage after esophagectomy. Eur J Surg Oncol. 2018;44(5):587-593.

17. Borggreve AS, Meijer JMR, Schreuder HWR, et al. Simulation-based trauma education for medical students: A review of literature. Med Teach. 2017;39(6):631-638.

18. Lam MG, Borggreve AS, Landman AJ, et al. Accessory Left Gastric Artery from Left Hepatic Artery is a Potential Risk for Radioembolization Complications. Cardiovasc Intervent Radiol. 2016;39(9):1367-8.

19. Borggreve AS, Landman AJEMC, Vissers CMJ, et al. Radioembolization: Is Prophylactic Embolization of Hepaticoenteric Arteries Necessary? A Systematic Review. Cardiovasc Intervent Radiol. 2016;39(5):696-704. 


\section{Acknowledgements (Dankwoord)}

De afgelopen jaren heb ik het voorrecht gehad om samen te werken met veel inspirerende mensen. Ik heb met volle teugen genoten van mijn tijd als arts-onderzoeker en er samen met jullie alles uit gehaald wat er in zat - niet in de laatste plaats buiten het ziekenhuis. Dit proefschrift is tot stand gekomen mede dankzij de directe en indirecte bijdrage van velen. De onderstaande personen wil ik graag in het bijzonder bedanken.

In de eerste plaats de patiënten met slokdarmkanker die geheel belangeloos hebben deelgenomen aan de onderzoeken beschreven in dit proefschrift in een periode die van hen en hun naasten sowieso al het uiterste vraagt. Het is bewonderenswaardig dat jullie als reden voor deelname aan onderzoek slechts de hoop uitspraken dat de behandeling en prognose van patiënten met slokdarmkanker ná jullie beter zou worden. Ik hoop dan ook dat het onderzoek beschreven in dit proefschrift daar op enige manier aan zal bijdragen.

Prof. dr. van Hillegersberg, beste Richard, je kritische blik, humor, vertrouwen en vermogen altijd twee stappen vooruit te denken creëren een omgeving waarin iedereen wordt gestimuleerd het beste uit zichzelf te halen. Ik ben dankbaar voor de ontwikkelingen die ik de afgelopen jaren onder jouw begeleiding heb mogen doormaken van student onderzoeker tot doctor. Het podium en de vrijheid die je aan je onderzoekers geeft waardeer ik enorm. Met veel plezier maak ik deel uit van je fantastische onderzoeksgroep!

Prof. dr. Terhaard, beste Chris, ik ben je erg dankbaar voor de kansen en de ruimte die jij en de afdeling mij hebben geboden gedurende mijn promotietraject. Bovendien waren je overkoepelende blik en de achtergrondinformatie die je verschafte over veel onderwerpen tijdens mijn voortgangsbesprekingen erg waardevol voor ons onderzoek.

Prof. dr. Ruurda, beste Jelle, toen ik in 2014 een email stuurde dat ik als student onderzoek wilde gaan doen, kreeg ik meteen een enthousiast en uitnodigend bericht terug. Datzelfde enthousiasme, je gevoel voor humor en je toegankelijkheid hebben onderzoek doen de afgelopen jaren leuk gemaakt! Heel veel dank bovendien voor jouw eerlijkheid, pragmatische instelling en altijd snelle reacties. Jij weet overal mogelijkheden te zien en te creëren, en de toewijding waarmee je de kliniek met de wetenschap weet te combineren is een voorbeeld.

Dr. van Rossum, beste Peter, je gedetailleerde revisies op manuscripten en je uitgebreide kennis over de literatuur op het gebied van slokdarmkanker hebben het onderzoek beschreven in dit proefschrift naar een hoger plan gebracht. Bedankt ook voor je kritische vragen die mij vaak uitdaagden om verder te denken! 
Leden van de beoordelingscommissie, prof. dr. P.A. de Jong, prof. dr. ir. J.J.W. Lagendijk, prof. dr. M.R. Vriens, prof. dr. J.J.B. van Lanschot en prof. dr. M. Verheij, hartelijk dank voor jullie interesse en de tijd die jullie hebben genomen om mijn proefschrift te beoordelen. Ik heb met veel plezier met jullie samengewerkt gedurende verschillende onderdelen van mijn promotie en ik kijk er naar uit om met jullie van gedachten te wisselen over de inhoud van mijn proefschrift.

Collega's van de oesmaag-onderzoeksgroep, Hylke Brenkman, Gijs van Boxel, Ingmar Defize, Emma Gertsen, Lucas Goense, Sylvia van der Horst, Feike Kingma, Kevin Parry, Bernadette Schurink, Maarten Seesing, Pieter van der Sluis, Arjen van der Veen, Roy Verhage, Els Visser en Teus Weijs, eendracht maakt macht! Ik had me geen leukere, meer inspirerende of meer betrokken onderzoeksgroep kunnen wensen. Het is een voorrecht om onderzoek te mogen doen met collega's waarvan je weet dat ze altijd voor je klaar staan, of dat nou tijdens de researchmeeting, op congressen, in de kelder of aan de bar is! Lucas, wat heb ik veel geleerd, maar vooral ook veel gelachen met jou als kamergenoot! Bedankt voor je eerlijke adviezen en de eindeloze gesprekken die we hebben gevoerd over de (on) zin van de wetenschap en het leven. Feike, tegelijkertijd gestart met onze promotietrajecten en sindsdien altijd slechts one call away. Jouw goede humeur en eerlijke humor, als ook jouw behulpzaamheid en bereidheid mee te denken met bijna elk onderdeel in dit proefschrift hebben er voor gezorgd dat wij vele uren met elkaar hebben doorgebracht. Wat mooi dat je vandaag naast me staat als paranimf!

Oesophagus-MRI onderzoekers, Gert Meijer, Stella Mook, Sophie Heethuis, Sophie de Mol van Otterloo, Mick Boekhoff en Astrid van Lier, bedankt voor jullie samenwerking en suggesties die onze onderzoekslijn vorm hebben gegeven. Gert, jouw laagdrempeligheid en oog voor detail maken je een unieke onderzoeker van wie ik veel heb kunnen leren. Stella, jouw kennis is mijn onderzoek op vele manieren ten goede gekomen, bedankt voor je betrokkenheid en fantastische gevoel voor humor. Sophie, zonder jou was ik nu nog steeds scans aan het intekenen - bedankt voor al je scripts en uitleg over MRI scans.

Arts-onderzoekers, arts-assistenten en stafleden van de heelkunde uit het UMC Utrecht, hartelijk dank voor alle inspirerende en onvergetelijke wetenschapsdagen, congressen, skireizen, borrels en weekenden. Het was altijd weer schitterend en ik ben blij dat ik weer in jullie midden ben!

Arts-onderzoekers van de afdeling radiotherapie, bedankt voor jullie gezelligheid, behulpzaamheid, de journal clubs - extra leerzaam dankzij de toewijding van prof. dr. Lenny Verkooijen -, lunches, weekstarts en het luisterend oor. In het bijzonder wil ik mijn 
kamergenoten - Lucas Goense, Sofie Gernaat, Jeanine Vasmel en Bart Pielkenrood - en mijn buren op de Q2 gang bedanken: door jullie ben ik geen dag met tegenzin naar mijn werk gegaan! Sofie, de manier waarop jij in het leven staat is voor mij enorm inspirerend, bedankt!

Dames van het secretariaat van de heelkunde - Romy, Marielle en Fatiha -, het secretariaat van de radiotherapie - Yvette, Judith en Leonie -, het afsprakenbureau en de (MRI) laboranten: jullie ondersteuning was onmisbaar bij de totstandkoming van dit proefschrift, bedankt voor jullie inzet!

Dr. Roks, beste David, bedankt voor jouw interesse en advies gedurende dit promotietraject. Je betrokkenheid bij mijn carrière en alles daarbuiten sinds je begeleiding van mij als semiarts in 2015 waardeer ik zeer!

Chirurgen en collega's uit het Academisch Ziekenhuis Paramaribo, met veel plezier heb ik mijn eerste maanden als arts-assistent bij jullie doorgebracht. Bedankt voor alles wat ik heb mogen doen en leren onder jullie supervisie en dat jullie me thuis hebben laten voelen! Ik hoop van harte dat we in de toekomst opnieuw samen zullen werken.

Lieve vrienden, clubgenoten, oud-huisgenoten, studiegenoten, hockeyteamgenoten en Paramaribo-gang, bedankt voor de ontelbare mooie momenten buiten het UMC de afgelopen jaren! Jullie loyaliteit, belangstelling en gezelligheid waardeer ik enorm. Op naar nog vele momenten samen! In het bijzonder wil ik graag Eva Bindels, Sanne Bergmans, Tabitha Koops en Floor Sijbrandij bedanken: jullie vriendschap is voor mij al meer dan 10 jaar onmisbaar en brengt me zo veel goeds! En last but not least, Martine Hendriksma, betrokken vanaf dag één van onze studie terwijl je plekken voor me bezet hield in de collegebanken, tot nu als paranimf. Bedankt voor de gesprekken over promoveren, je inspirerende doorzettingsvermogen, je vrolijkheid en natuurlijk je gezelligheid!

Lieve Armanda en Juliette, er is niets mooier dan opgroeien met jullie als zussen! Vanaf mijn geboorte hebben jullie me bedolven onder liefde, gezelligheid, eerlijkheid en grappen. Dat jullie onvoorwaardelijk voor me klaar staan - in Utrecht, Den Haag, San Diego, op de piste, op de Kilimanjaro of waar dan ook - betekent heel veel voor me. Ik kijk uit naar de mooie momenten die ons de rest van ons leven nog te wachten staan! Lieve Viren, Arth en Emile - ik kan me geen betere $x$-tended family wensen en prijs me ontzettend gelukkig met jullie allen om me heen! 
Lieve papa en mama, zonder jullie had ik dit proefschrift - zowel letterlijk als figuurlijk - nooit kunnen schrijven. Door jullie onvoorwaardelijke steun en liefde, grenzeloze vertrouwen en kritische blik hebben jullie mij het beste kompas gegeven om door het leven te navigeren en mijn dromen te verwezenlijken. De afgelopen jaren waren we vaak ver van elkaar verwijderd in geografische zin, maar dat jullie mijn grootste supporters zijn heb ik altijd geweten! Ik ben jullie oneindig dankbaar. 


\section{Curriculum vitae auctoris}

Alicia Sabine Borggreve was born on March 8, 1992 in Venray, the Netherlands. At the age of 9, Alicia moved to Waalre with her parents and 2 older sisters. After graduating (cum laude) from the Pleincollege Van Maerlant, Eindhoven, she started medical school at the University of Utrecht in 2009. During her studies, she was an active member of several student organizations and taught practical skills to undergraduate medical students. Furthermore, Alicia gained international experience during clinical internships at Tygerberg Hospital (Stellenbosch University, South Africa) and the University of

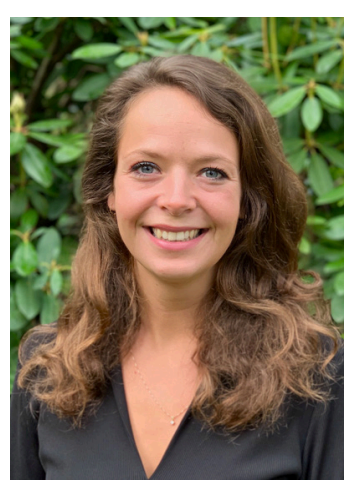
California (San Diego, United States of America). In the final years of her studies, she developed a special interest in general surgery and started her first project on esophageal cancer as a student researcher in 2014.

After obtaining her medical degree in 2016, Alicia started as a PhD candidate focusing on multimodality treatment and response assessment in esophageal cancer at the Departments of Surgery and Radiation Oncology of the University Medical Center Utrecht, under supervision of prof. dr. R. van Hillegersberg, prof. dr. C.H.J. Terhaard, prof. dr. J.P. Ruurda and dr. P.S.N. van Rossum. In parallel with her PhD program, she obtained a postgraduate Master of Science degree in Epidemiology at the University of Utrecht specializing in Clinical Epidemiology and Medical Statistics.

Alicia started her clinical career as a surgical resident not in training at the Department of Surgery at the Academic Hospital Paramaribo, Surinam in July 2019. In February 2020, she returned to the University Medical Center Utrecht as a surgical resident not in training. Alicia aspires to become a surgeon while maintaining her passion for research and healthcare in low-resource settings. 


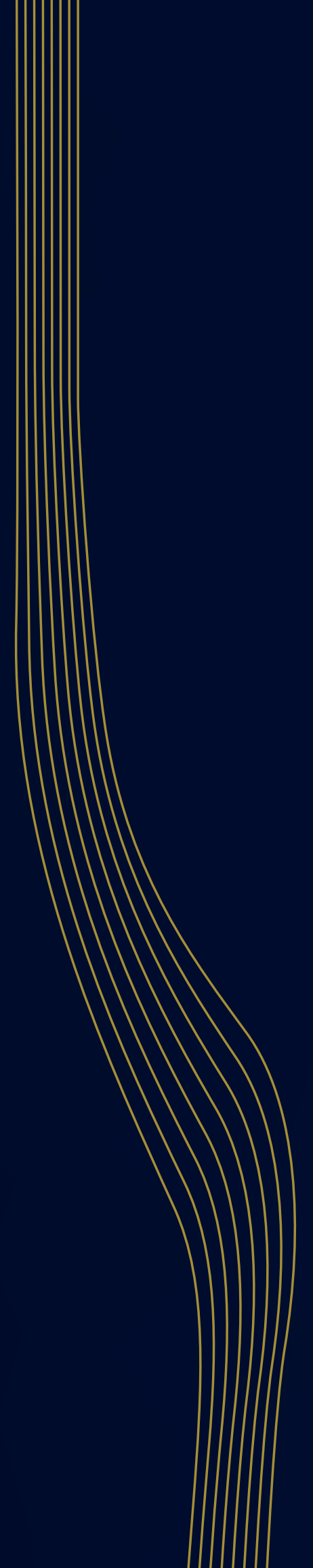

